\title{
Characterizing Cobalamin Cycling by Antarctic Marine Microbes across Multiple Scales
}

\author{
by \\ Deepa Rao \\ S.B., Earth, Atmospheric, and Planetary Sciences, \\ Massachusetts Institute of Technology, 2012 \\ M.A., Communication, Culture, \& Technology, \\ Georgetown University, 2014 \\ Submitted in partial fulfillment of the requirements for the degree of \\ Doctor of Philosophy \\ at the \\ MASSACHUSETTS INSTITUTE OF TECHNOLOGY \\ and the \\ WOODS HOLE OCEANOGRAPHIC INSTITUTION
}

May 2020

(C) Deepa Rao, 2020. All rights reserved.

The author hereby grants MIT and WHOI permission to reproduce and to distribute publicly paper and electronic copies of this thesis document in whole or in part in any medium now known or hereafter created.

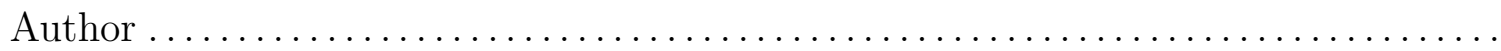

MIT-WHOI Joint Program in Oceanography/Applied Ocean Science and Engineering Massachusetts Institute of Technology and Woods Hole Oceanographic Institution

May 21st, 2020

Certified by

Dr. Michael J. Follows

Chair, Joint Committee for Biological Oceanography Massachusetts Institute of Technology

Thesis Supervisor

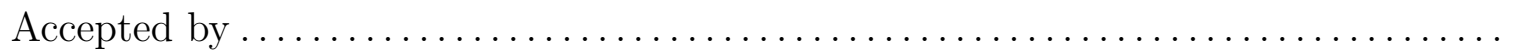

Dr. Michael J. Follows

Chair, Joint Committee for Biological Oceanography Massachusetts Institute of Technology 
I dedicate this thesis to my loving family who have continued to inspire me to aim high, work hard, and always be curious. 


\title{
Characterizing Cobalamin Cycling by Antarctic Marine Microbes across Multiple Scales \\ by \\ Deepa Rao
}

\author{
Submitted to the MIT-WHOI Joint Program in Oceanography/Applied Ocean Science and \\ Engineering on May 21st, 2020, \\ in partial fulfillment of the requirements for the degree of \\ Doctor of Philosophy in Biological Oceanography
}

\begin{abstract}
Highly productive marine microbial communities in the coastal Southern Ocean sustain the broader Antarctic ecosystem and play a key role in Earth's climate via the biological pump. Regional phytoplankton growth is primarily limited by iron and co-limited by cobalamin (vitamin $\mathrm{B}_{12}$ ), a trace cobalt-containing organometallic compound only synthesized by some bacteria and archaea. These micronutrients impact primary production and the microbial ecology of the two keystone phytoplankton types: diatoms and Phaeocystis antarctica. This thesis investigates microbe-driven cobalamin cycling in Antarctic seas across multiple spatiotemporal scales. I conducted laboratory culture experiments with complementary proteomics and transcriptomics to investigate the $\mathrm{B}_{12}$-ecophysiology of $P$. antarctica strain CCMP 1871 morphotypes under iron- $\mathrm{B}_{12}$ co-limitation. We observed colony formation under higher iron treatments, and a facultative use of $\mathrm{B}_{12}$-dependent (MetH) and $\mathrm{B}_{12}$-independent (MetE) methionine synthase isoforms in response to vitamin availability, demonstrating that this strain is not $\mathrm{B}_{12}$-auxotrophic. Through comparative 'omics, we identified a putative MetE protein in $P$. antarctica abundant under low $\mathrm{B}_{12}$, which is also found in other marine microbes. Across Antarctic seas, community-scale cobalt and $\mathrm{B}_{12}$ uptake rates were measured by ${ }^{57} \mathrm{Co}$ radiotracer incubation experiments and integrated with hydrographic and phytoplankton pigment data. I observed significant correlations between uptake fluxes and environmental variables, providing evidence for predominantly diatom-driven uptake of these micronutrients in warmer, fresher surface waters with notable regional differences. To date, this work is the most comprehensive attempt to elucidate the processes governing the co-cycling of cobalt and $\mathrm{B}_{12}$ in any marine system. At the ecosystem-scale, I developed and tested a hypothesis of micronutrient-driven community dynamics through a trait-based model with cross-feeding interactions. The model demonstrates how the observed seasonal succession of springtime $P$. antarctica from solitary to colonial cells, bacterioplankton, and summertime diatoms may be explained by the microbial cycling of iron, dissolved organic carbon, and $\mathrm{B}_{12}$. Overall, this dissertation provides new information about the micronutrient-driven ecology of Antarctic marine microbes and adds to our understanding of the interconnections between organismal life cycle, trace metals, and trace organics in marine environments.
\end{abstract}

Thesis Supervisor: Dr. Michael J. Follows

Title: Professor, Massachusetts Institute of Technology 


\section{Acknowledgments}

My training as a scientist during my time in the MIT-WHOI Joint Program (20142020) and the work presented in this dissertation were financially supported by the Academic Programs Office (APO) at the Woods Hole Oceanographic Institution (WHOI) and various funding agencies. My first semester was supported by the WHOI Von Damm Fellowship (2014). Subsequent years and endeavors were supported by awards from the Gordon and Betty Moore Foundation to Professor Michael Follows (Award 3778, M.J.F.) and Simons Collaboration on Computational Biogeochemical Modeling of Marine Ecosystems (Award 549931, M.J.F.); National Science Foundation (NSF) grant to Dr. Stephanie Dutkiewicz (Grant number 1434007, S.D.), and NSF Office of Polar Program (OPP) grant to Dr. Makoto Saito (M.S.) for the CICLOPS research expedition (OPP-1643684, OPP-1643845, and OPP-1644073).

I appreciate the institutional resources provided by MIT and its Department of Earth, Atmospheric, \& Planetary Sciences (EAPS), and by WHOI and its Department of Biological Oceanography and Department of Marine Chemistry \& Geochemistry. The generous support of these institutions along with travel grants afforded me the opportunities to learn from, engage with, and become part of a global scientific network. Thank you to the University of Hawai'i, Center for Microbial Oceanography: Research and Education (C-MORE), and Augoron Institute for funding my participation in the 2015 Summer Course on Microbial Oceanography. This was my first experience conducting science at sea. Thank you to the Gordon Research Conferences for supporting my attendance at the Marine Microbes meetings in 2016 and 2018. I look forward to being the Co-Chair of the Gordon Research Seminar for Marine Microbes in our rescheduled meeting in 2022. I am grateful for the opportunities afforded to me to attend several summer courses and conferences in microbial ecology, oceanography, complex systems, and science communication during my dissertation. In particular, my summers spent at the C-MORE Summer School for Oceanography (2015) in Hawai'i and the Santa Fe Institute's Complex Systems Summer School in New Mexico broadened my horizons. One of the most rewarding and enriching experiences of my Ph.D. has been the opportunity to become part of a global network of Earth scientists. When I took my first steps on Antarctica at McMurdo Station, I did not expect to run into several friends and colleagues from various stages in my scientific career. A special thanks to MIT and WHOI for giving creative minds room to grow, connect, and share resources to study and solve some of the most complex challenging of our time, especially when it comes to understanding our planet and its changing climate.

A huge thank you to the incredible administers and members of the collective MIT EAPS and WHOI communities. I would like to especially acknowledge the Deans, Department Heads, and current and former members of EAPS, its science writers, and staff in headquarters, including Helen Hill, Roberta Allard, Jane Abbot, Kris Kipp, Vicki McKenna, Jennifer Fentress, Lauren Hinkel, Brandon Milardo, Darius Collazo, Angela Ellis, and Maggie Cedarstrom. A huge thanks to the staff at WHOI's APO, 
especially Julia Westwater and Lea Fraser, as well as Mary Zawoysky for helping us students navigate the Joint Program and all of its quirks. Thank you for making these environments a welcome place for so many of us.

It has hard to believe that 10 years ago, I decided to become an EAPS undergraduate major after taking an eye-opening field course with Professors Tim Grove and Sam Bowring. I am so glad to have been an undergraduate researcher in the lab of Professor Ron Prinn, working with (now) Professor Laura Meredith in the labs of Professors Bosak and Ono for my senior year research project. It is thanks to Professor Penny Chisholm and her undergraduate ecology course that I found my place at MIT and learned that I could combine research in microbial ecology, biogeochemistry, and climate in an interdisciplinary way to understand the Earth system. Although EAPS is a small department, it has been an incredible environment in which to learn, grow, and contribute to during my decade at MIT. Around 5 years ago I also became part of the MIT-WHOI community. Thank you to Dr. Amala Mahadevan for welcoming me as a guest student in 2014 and for being a wonderful guide, confidant, and mentor throughout my time at WHOI as my summer advisor and finally my thesis defense chair in 2020. Finally, I would like to thank the maintenance and custodial staff for not only keeping the institutes functioning, but for making me feel safe during many late nights working alone at MIT and WHOI.

I would like to thank my advisor Professor Mick Follows for his guidance, wisdom, support, optimism, and mentorship over these past $6+$ years since I first emailed him to learn more about microbial oceanography. I remain as inspired by the Darwin model that you and Dr. Stephanie Dutkiewicz developed as the day I first read that publication. Mick, you gave me the opportunity of a lifetime to pursue this dissertation and spend my days examining and understanding our biosphere and the complex network of marine microbes that shapes our living planet. Having your encouragement throughout this process gave me the courage I needed to continue even when the path forward was unclear. I will always look fondly back on our many sessions puzzling over equations quickly scribbled on your white board as to never let an enticing idea escape, conversations about the global ramifications of the world's smallest organisms, and our mutual excitement over good science writing and art. Thank you for trusting in me, and giving me the creative freedom to develop the research in this dissertation. I have learned so much from you and look forward to applying this knowledge throughout my career.

I also want to thank Dr. Mak Saito who welcomed me into his lab group and became an incredible mentor for a large portion of my dissertation. Mak, since I first contacted you to discuss my thesis proposal, it was clear that you have a positive spirit, collaborative mindset, and fearless approach to tackling scientific problems. I have enjoyed observing your mentorship style and approach in the lab, whereby you encourage everyone and embrace experimentation, challenging questions, and exploration. Thank you for the opportunity to learn new techniques, and especially for integrating me into your group for the CICLOPS research cruise to Antarctica. It has been a joy to be part of your lab and I am excited for the scientific discoveries that await in all that data.

Next, I want to acknowledge my thesis committee, which also includes Dr. Stephanie 
Dutkiewicz, Professor Serguei Saavedra, and Professor Alex Worden. Steph, thank you for your many helpful discussions on numerical methods, phytoplankton physiology, ecological modeling, and for your advise and feedback throughout my dissertation's development. A special thanks to Serguei and Alex, who have provided critical feedback on the research presented in this thesis. Alex, I look forward to collaborating more to examine our much-loved multifarious marine microbes. I have enjoyed working with all of you and learning how to approach problems from your unique perspectives.

I also want to acknowledge my past mentors over the course of my scientific training. Thank you to my undergraduate mentor Professor Laura Meredith for introducing me to field microbial ecology and the Harvard Forest, which holds a special place in my heart; Professor David Ribes for introducing me to the field of Science, Technology, and Society and to the gateway book Alien Ocean, which lead me on this journey into microbial oceanography; Professor Linda Garcia who advised me during my M.A. in Georgetown and who encouraged my scholarly examination of the integration of science and art for education; and Catherine Smith and J. Bob Cook, my earth science and biology teachers from middle school and high school who always went the extra mile to share the wonders of the natural world with their students in creative ways and with an excess of witty puns.

I am grateful to have participated in the CICLOPS research cruise aboard the R/V Nathaniel B. Palmer as we sampled Antarctic coastal environments from the Amundsen Sea to Ross Sea and Terra Nova Bay. Without the captain, crew, and Antarctic Support Staff, our scientific research would not be possible - and the cruise would have been far less entertaining. A special thank you to Jamee Johnson for her help in keeping our scientific research equipment up and running and to Matt Pullen for being a great companion during sleepless, sunlit Antarctic nights filled with restless emperor penguins waiting to discovered. Finally, thank you to our chief scientist Professor Jack DiTullio for rallying the crew and scientists with optimism and humor, being a wealth of knowledge in Antarctic ecosystems, and for generally being a great role model.

I would like to thank all the members of the Follows and Saito Labs - former and current - for being excellent colleagues in the pursuit of science. A special thanks to Emily Zakem, Jon Lauderdale, Chris Follett, Rogier Braakman, David Talmy, Greg Britten, and Zhen Wu for many helpful and insightful discussions on modeling microbial metabolisms. To the newest members of the Follows Lab, Ari Krinos and Lexi Jones, I am excited to see the wonderful directions that your research will lead you to. I would also like to thank Dawn Moran, Matt McIlvin, Marissa Kellogg, Natalie Cohen, Becca Chimel, and Mike Mazzotta for sharing their tacit lab knowledge with me as I learned trace metal clean methods, Antarctic algal culturing secrets, proteomics, and bioinformatics analyses. I especially thank Dawn for training me in the early days of my algal culture work and her crucial help in experiments, as well as Matt for being the resident mass spectrometry guru. Science is an inherently collaborative endeavor and I could not ask for better colleagues and mentors.

To my friends at MIT, WHOI, Cambridge, and beyond, thank you for being an inspiring, hilarious, and supportive lot of clever and caring humans. A special thanks to Sam Fried, Fatima Hussain, Laura Weber, Casey Zakroff, Anya Sharifo-Birger, Ari 
Schlesinger, Julia Titarelli, Mara Freilich, Margaret Duffy, Mukund Gupta, Rohini Shivamoggi, Hanny Rivera, Jenny Panlilio, Megan May, Emily Zakem, Cherry Gao, and so many more friends and colleagues around the world. Some of my favorite memories in the Joint Program outside of the lab are playing games, traveling, cooking, painting, hiking, riding bikes, musing, and laughing with you all. To my office mates over the years, especially the cohort of 54-1519, thanks for making coming to work enjoyable, full of interesting conversations, and overly ambitious workout challenges. I also want to share how heartwarming of an experience it was to see the 100+ smiling faces of so many people from different aspects of my life join for my online dissertation defense in the midst of a global pandemic. Thank you to my community, near and far.

Last but not least, I thank my loving extended family for their never-ending support, especially my parents, Drs. Ramachandra and Shashikala Rao, and my husband Dr. Robert Haussman. This Ph.D. is possible because of your support, humor, and many cups of morning coffee and midnight chai. Thank you for making this dream of mine a reality. Mom and Dad, thank you inspiring me to always grow, set lofty goals, and value the friends and family that make life a joy to share. You have and always will be with me throughout my all of life's adventures. Even when I was in the Antarctic, I was fortunate enough to be able to send you photos and share a part of that incredible experience with you all. Mom and Dad, you inspire me to strive towards the best version of myself so I can extend the love and support you have given me.

Robert, you are the greatest addition to my life. I could not imagine a more brilliant, kindhearted, understanding, and wonderfully silly person to build a life with. Thank you for your support, $\mathrm{AT}_{\mathrm{E}} \mathrm{X}$ wizardry, and patience throughout this dissertation process. Along with you comes wonderful and loving parents, Ken and Teresa Haussman, sisters Nicole and Amanda, and great nieces and nephews. Thank you to the Haussmans for welcoming me with open arms into the family. Our wedding last year was the happiest period of my life, and I am so thankful to have grown our multicultural family and brought together more friends and family from around the world to celebrate the occasion. Our many conversations about science, Mother Earth, and the cosmos have expanded my mind and heart. Your support throughout this Ph.D. has allowed me to grow as a scientist and as your loving spouse. We have had quite the adventure so far together and I look forward to all our future holds.

Over the course of this dissertation, there have been several difficult periods, but thankfully more occasions to celebrate victories, small and big. I made it through those challenging times with the support of my family. Despite being thousands of miles apart from my parents and husband, across different states, opposite ends of the country, and even at the bottom of the world, they have always been a phone call away. I am glad that I can now add another Dr. Rao to our family. 


\section{Contents}

$\begin{array}{ll}\text { List of Figures } & 13\end{array}$

$\begin{array}{ll}\text { List of Tables } & 15\end{array}$

$\begin{array}{ll}\text { Chapter 1: Introduction } & 17\end{array}$

1.1 Motivation . . . . . . . . . . . . . . . . . . . . . . 17

1.2 Background . . . . . . . . . . . . . . . . . . . 18

1.3 Thesis Overview . . . . . . . . . . . . . . . . . . . . . . . . 24

Chapter 2: Phaeocystis antarctica has a flexible vitamin $\mathrm{B}_{12}$ ecophysiology: A multi-omic study reveals its response $\begin{array}{ll}\text { to iron and cobalamin stress } & 29\end{array}$

Abstract . . . . . . . . . . . . . . . . . . 30

2.1 Introduction . . . . . . . . . . . . . . . . . . 30

2.2 Methods . . . . . . . . . . . . . . . . . . . . . . . . 33

2.3 Results . . . . . . . . . . . . . . . . . . . . . . . . . . 39

2.4 Discussion . . . . . . . . . . . . . . . . . . . . . 58

2.5 Conclusion . . . . . . . . . . . . . . . . . . . . . . . . . . . 61

2.6 Supplemental Materials . . . . . . . . . . . . . . . . . . 63

Chapter 3: Patterns of microbial community driven cobalt $\begin{array}{ll}\text { and cobalamin uptake in Antarctic seas } & 69\end{array}$

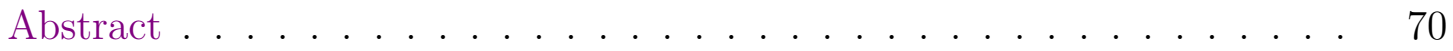

3.1 Introduction . . . . . . . . . . . . . . . 71

3.2 Materials \& Methods . . . . . . . . . . . . . . . . . . . . . 73

3.3 Results . . . . . . . . . . . . . . . . . . . . 75

3.4 Discussion . . . . . . . . . . . . . . . . . . . . . . 96

3.5 Conclusion . . . . . . . . . . . . . . . . . . . . . . . . . . . . . . . 103

3.6 Supplemental Materials . . . . . . . . . . . . . . . . . . . . . . . . . . 104

Appendices . . . . . . . . . . . . . . . . . . . 110

3.A Ecological cobalt and cobalamin cycles in Antarctic marginal seas . . 110

3.B Water mass characterization of CICLOPS expedition samples ... . 113 
Chapter 4: A model of micronutrient-driven seasonal succession $\begin{array}{ll}\text { of Antarctic marine microbial communities } & 117\end{array}$

Abstract . . . . . . . . . . . . . . . . . . . . . . . 118

4.1 Introduction . . . . . . . . . . . . . . . . . . . 118

4.2 Methods: Model Development . . . . . . . . . . . . . . . . . . . 126

4.3 Results \& Analysis . . . . . . . . . . . . . . . . . . . . . 135

4.4 Conclusion . . . . . . . . . . . . . . . . . . . . . . . . . 143

4.5 Next steps in model development . . . . . . . . . . . . . . . . 144

4.6 Supplemental Figures . . . . . . . . . . . . . . . . . . . . . . . . . . . 149

$\begin{array}{ll}\text { Chapter 5: Conclusion and Future Directions } & 151\end{array}$

5.1 Thesis Overview . . . . . . . . . . . . . . . . . . . . . . . . . . . . . 151

5.2 Dissertation Findings . . . . . . . . . . . . . . . . . . . . . 153

5.3 Broader Implications and Future Outlook . . . . . . . . . . 157

$\begin{array}{ll}\text { Bibliography } & 161\end{array}$ 


\section{List of Figures}

1-1 Chemical structure and variants of cobalamin . . . . . . . . . 20

$1-2$ Research strategy . . . . . . . . . . . . . . . . . . 25

2-1 P. antarctica morphotype ratios in Fe- $\mathrm{B}_{12}$ culture treatments . . . . 40

2-2 $\quad$ P. antarctica growth rate and cell density at time of harvest . . . . . 41

2-3 PCA analysis of culture transcriptomes . . . . . . . . . . . 42

2-4 Methionine synthase isoform gene expression and protein abundance

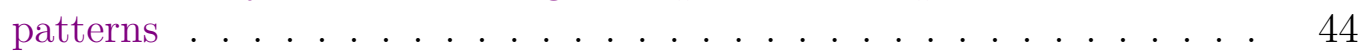

2-5 Venn diagram of significantly differentially abundant proteins between

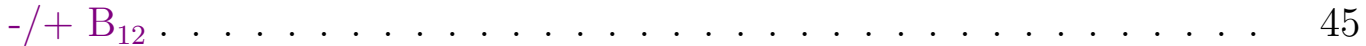

2-6 $\quad$ C1 metabolism proteins affected by $\mathrm{B}_{12} \ldots \ldots \ldots \ldots \ldots$

2-7 Scatter plot of differentially abundant proteins under $-/+\mathrm{B}_{12}$, low iron 50

2-8 Scatter plot of differentially abundant proteins under $-/+\mathrm{B}_{12}$, high iron 51

2-9 MA plots of $P$. antarctica proteomes in response to $-/+$ Fe and $\mathrm{B}_{12}$. 52

2-10 Two putative MetE protein contigs . . . . . . . . . . . 54

2-11 Putative MetE protein domains . . . . . . . . . . . . . 55

2-12 Phylogenetic tree of putative MetE protein . . . . . . . . . . 57

2-13 Growth curves of P. antarctica cultures . . . . . . . . . . . 63

2-14 Heme biosysnthesis pathway in responsive to $-/+\mathrm{B}_{12} \ldots \ldots \ldots 6$

2-15 Detailed putative MetE portein domain . . . . . . . . . . 66

2-16 Protein sequence of in silico putative MetE construct . . . . . . . 66

2-17 Phylogenetic tree of putative MetE protein without the canonical diatom MetE . . . . . . . . . . . . . . . . . . . . 67

3-1 CICLOPS research expedition stations where radiochemical uptake rates were measured . . . . . . . . . . . . . 76

3-2 Vertical profiles of the Amundsen-Ross Transect: Hydrographic properties, ${ }^{57} \mathrm{Co}$ and ${ }^{57} \mathrm{~B}_{12}$ uptake rates, and $\mathrm{Co}_{\text {total }} \ldots \ldots \ldots . . .77$

3-3 Water masses of the Amundsen Sea, Ross Sea, Terra Nova Bay, Antarctica 78

3-4 CICLOPS phytoplankton chemotaxonomic pigment concentrations . . 80

3-5 Size-fractionated surface percent ${ }^{57} \mathrm{Co}$ uptake rate . . . . . . . . . 84

3-6 Size-fractionated surface Co uptake rate . . . . . . . . . . . . 84

3-7 Total Co vs. $\%{ }^{57}$ Co uptake rate for the Amundsen-Ross Transect . . 85

3-8 Size-fractionated surface percent ${ }^{57} \mathrm{~B}_{12}$ uptake rate . . . . . . . . . 86

3-9 Bacterioplankton fractional contribution to ${ }^{57} \mathrm{~B}_{12}$ uptake at Station $15 \quad 88$ 
3-10 Relationship of ${ }^{5}{ }^{57} \mathrm{Co}$ and ${ }^{57} \mathrm{~B}_{12}$ uptake with salinity . . . . . . . 90

3-11 Diatom bloom in Station 22 detected by chemical proxies . . . . . . . 92

3-12 Profiles of radiochemical uptake measurements in stations along the Amundsen-Ross Transect . . . . . . . . . . . . . . . 93

3-13 Percent uptake rates of ${ }^{57} \mathrm{Co}$ and ${ }^{57} \mathrm{~B}_{12}$ vs. chemotaxonomic diatom and P. antarctica pigments . . . . . . . . . . . . .

3-14 Percent ${ }^{57} \mathrm{Co}$ uptake rate vs. chemotaxonomic pigments in the Amundsen-Ross Transect . . . . . . . . . . . . . . . . 95

3-15 Cobalt uptake rates vs. chemotaxonomic pigment concentrations . . . 97

3-16 Regional correlations between $\mathrm{Co}_{\text {total }}$ and phytoplankton pigments . . 98

3-17 Regional correlations between ${ }^{57} \mathrm{Co}$ uptake and P. antarctica indicator pigment 19-Hex . . . . . . . . . . . . . . .

3-18 Percent ${ }^{57} \mathrm{~B}_{12}$ uptake vs. diatom indicators fucoxanthin and biogenic silica . . . . . . . . . . . . . . . . . . . 100

3-19 A hypothesis of micronutrient-driven seasonal succession in coastal Antarctic marine microbial communities . . . . . . . . . . . . 101

3-20 Map of all stations during CICLOPS (NBP-1801) cruise . . . . . . 105

3-21 Profiles of percent ${ }^{57} \mathrm{~B}_{12}$ uptake in the total community ( $\geq 0.2 \mu \mathrm{m}$ size fraction) . . . . . . . . . . . . . . . 106

3-22 Profiles of percent ${ }^{57} \mathrm{Co}$ uptake in the total community ( $\geq 0.2 \mu \mathrm{m}$ size fraction) . . . . . . . . . . . . . . . . . 107

3-23 The coastal Antarctic marine ecological cycle of cobalt and $\mathrm{B}_{12} \ldots \ldots$

3-24 Water mass characterization of CICLOPS CTD measurements . . . . 114

3-25 Water masses of the Amundsen Sea, Ross Sea, Terra Nova Bay with neutral density values . . . . . . . . . . . . . . . . 115

3-26 Surface water masses of the Amundsen Sea, Ross Sea, Terra Nova Bay 116

4-1 Life cycle transitions in P. antarctica and seasonal abundance in the Ross Sea, Antarctica . . . . . . . . . . . . . . . . . . . . . 122

4-2 Model framework . . . . . . . . . . . . . . . . . . . . . . 127

4-3 Allometric scaling relationships . . . . . . . . . . . . . . 128

4-4 Resource concentration in the diffusive boundary layer . . . . . . . . 129

4-5 Function describing transition from $P$. antarctica solitary to colonial cells 133

4-6 Steady state model solutions with a set of fixed iron supply rates . . . 136

4-7 Iron supply rate in the seasonal model . . . . . . . . . . . . . . 138

4-8 Seasonal succession pattern of model microbial types . . . . . . . . 139

4-9 Seasonal succession pattern of model microbes and resources . . . . . 140

4-10 Seasonal cycle of model microbial populations and resources . . . . . 141

4-11 A hypothesis of micronutrient-limited seasonal succession in Antarctic marine microbial communities . . . . . . . . . . . . . . . . 144

4-12 Seasonal succession of microbial population with varying iron supply rate149 


\section{List of Tables}

3.1 Comparison of dissolved cobalt concentrations and percent ${ }^{57}$ Co uptake from CICLOPS and CORSACS . . . . . . . . . . . . . . . 82

3.2 Summary of potential cobalt and cobalamin uptake rates . . . . . . 89

3.3 Concentrations of dissolved cobalt chemical species during CICLOPS 108

3.4 Concentrations of dissolved cobalt chemical species during CICLOPS by region . . . . . . . . . . . . . . . . . . . . 109

4.1 Model variables and parameters . . . . . . . . . . . . . . . . 131

4.2 Model parameter values and allometric relationships . . . . . . . . . 148 


\section{Chapter 1}

\section{Introduction}

"It is interesting to contemplate an entangled bank, clothed with many plants of many kinds, with birds singing on the bushes, with various insects flitting about, and with worms crawling through the damp earth, and to reflect that these elaborately constructed forms, so different from each other, and dependent upon each other in so complex a manner, have all been produced by laws acting around us."

- Charles Darwin (On the Origin of Species by Natural Selection, or the Preservation of Favoured Races in the Struggle for Life, 1859, XIV:489)

\subsection{Motivation}

The global ocean microbiome is the emergent result of a complex network of ephemeral interactions in an ever-changing environment. Marine microbial ecosystems are driven by the primary production of phytoplankton, a diverse set of photosynthetic microbes comprising both cyanobacteria and eukaryotic microalgae. Although phytoplankton form the base of ocean food webs, drive the biogeochemical cycling of nutrients, and are responsible for nearly half of global carbon fixation, they are not metabolically independent (Droop, 1957; Field et al., 1998). Most microalgae (indeed most lifeforms on Earth) are auxotrophic for some biologically-derived metabolites, requiring an external source of these compounds to grow because they cannot synthesize them de novo. These essential metabolites include vitamins, co-factors, and amino acids, which are considered "trace organics" in seawater due to their low concentrations as a result of high flux through producers and consumers in the ecosystem. Nutritional auxotrophy is commonplace in natural microbial communities.

In particular, coexisting algae and bacteria interact in multifarious ways, often based on the synergistic exchange of metabolites and organic carbon (Worden et al., 2015). To fulfill their nutritional needs, marine microbes have adapted to acquire trace organics from their environment and often do so by engaging in loose syntrophic and cross-feeding interactions or close symbiotic relationships. Syntrophy is the "critical interdependency between producer and consumer," according to Dolfing (2014) and 
includes interactions that range from specific and close-knit (e.g., associated bacteria on an alga) to non-specific and distributed (e.g., metabolite exchanges within a mixed community). Classical ecological theory describes these nutrient-cycling interactions according to the successional exchange of electron donors. For example, the emergent Winodgradsky column from a collection of microbes self-assembling into an ecosystem structured along a redox reaction gradient. However, recent insights have broadened the scope of nutritional interactions between microbes to include nutrient competition, syntrophic exchange of metabolic byproducts, and cross-feeding of essential metabolites like vitamins and amino acids from prototrophs to auxotrophs (Seth and Taga, 2014; Zengler and Zaramela, 2018). Indeed, studies have determined that trace organics can affect community composition and primary primary production on regional scales (Bertrand et al., 2007; Gobler et al., 2007; Moore et al., 2013; Panzeca et al., 2006; Sañudo-Wilhelmy et al., 2006). Thus, microbial interactions involved in cycling organic resources are a vital component of the global carbon cycle that ultimately influence Earth's climate system.

Examining the network of ecological interactions, particularly those involved in cycling trace organics and overcoming resource limitation, can aid our understanding of the structure and function of marine microbial communities. By tugging at these "invisible threads" that connect marine microbes, we can start to reveal the complex network of interconnections that shape the microbial ocean. This thesis focuses on one thread - cobalamin, more commonly known as vitamin $\mathrm{B}_{12}$-and its cycling through Antarctic marine microbial ecosystems.

Vitamin $\mathrm{B}_{12}$ is a prime example of interspecies nutritional dependence in the global ocean. Unlike other metabolites, $\mathrm{B}_{12}$ is only synthesized de novo by bacterioplankton, yet most microalgae require this vitamin to grow. The Southern Ocean and productive Antarctic seas have ecological and environmental conditions that increase the potential for $\mathrm{B}_{12}$-limitation, with wide-ranging consequences for biogeochemistry and carbon cycle feedbacks. The research studies presented herein examines the Antarctic $\mathrm{B}_{12}$ system using three complementary approaches: (1) a laboratory study of the $\mathrm{B}_{12}$ ecophysiology of a keystone phytoplankton species Phaeocystis antarctica, (2) fieldbased in situ uptake rates of $\mathrm{B}_{12}$ and its metal center cobalt in Antarctic seas, and (3) a numerical model of micronutrient-driven seasonal succession of dominant Antarctic marine microbes based on iron and $\mathrm{B}_{12}$ dynamics.

\subsection{Background}

Understanding the potential impact of $\mathrm{B}_{12}$ in marine ecosystem requires one to start with the biochemistry of $\mathrm{B}_{12}$ and follow the threads that lead to an understanding of its ecological role. From prokaryotic origins, $\mathrm{B}_{12}$ is cycled in a complex way through an entire economy that has evolved to traffic the vitamin and connect producers to consumers and even those that salvage and recycle components of this costly molecule (Giovannoni, 2012).

$\mathrm{B}_{12}$ is an ancient molecule that has been an active part of life on Earth for billions of years. Biochemical and phylogenetic analyses date $\mathrm{B}_{12}$ to having evolved 2.7-3.5 
billion years ago in anaerobes in an Archaean Ocean that was low in oxygen and relatively high in trace metals like iron and cobalt. $\mathrm{B}_{12}$ is an intricate organometallic molecule whose function relies on its structure and the metal chemistry of a rare Co atom positioned in the center of a corrin ring (Fig.1-1). Cobalt a bio-essential metal that is primarily used in $\mathrm{B}_{12}$, despite being a trace element in Earth's crust (about $20 \mathrm{ppm}$ on average) and ocean (ranging from $10 \mathrm{pM}$ to $100 \mathrm{pM}$ ) (Carr and Turekian, 1961; Hazen et al., 2017; Saito et al., 2002, 2004, 2010). In seawater, $\mathrm{B}_{12}$ is even more scarce, measured in the range of $<1 \mathrm{pM}$ to $10 \mathrm{pM}$ as a result of environmental and ecological forcings (Sañudo-Wilhelmy et al., 2012).

$\mathrm{B}_{12}$ and its chemical variants act as nutrients, co-factors, and co-enzymes in concert with proteins to infer specialized enzymatic functions. In prokaryotes, $\mathrm{B}_{12}$ is used in several key metabolic processes, including carbon and nitrogen catabolism, one-carbon metabolism, methylation, and the synthesis of methionine, bacteriochlorophyll, and nucleotides; these uses are nicely reviewed in Shelton et al. (2019). In eukaryotes, $\mathrm{B}_{12}$ is primarily a co-factor used in methylmalonyl-CoA (MCM), and type II ribonucleotide reductase $(\mathrm{RNR} \mathrm{II})$, and $\mathrm{B}_{12^{-}}$dependent methionine synthase $(\mathrm{MetH})$. Another $\mathrm{B}_{12^{-}}$ independent isoform (MetE) of methionine synthase exists and will be discussed in detail in the following sections. The cobalt atom in the corrin ring changes its oxidation state, enabling unique carbon radical chemistry that is used in metalloenzymes and methyltransferases (Kräutler, 2005).

$\mathrm{B}_{12}$ is the largest and most structurally complex vitamin in nature. It is costly in terms of inorganic resources, genes, proteins, and energy to synthesize and assimilate. The chemical formula of cyanocobalamin $\left(\mathrm{C}_{63} \mathrm{H}_{88} \mathrm{CoN}_{14} \mathrm{O}_{14} \mathrm{P}\right)$ makes clear the large quantity of nitrogen atoms and a rare $\mathrm{Co}$; other co-factor forms of $\mathrm{B}_{12}$ require even more nitrogen. De novo $\mathrm{B}_{12}$ synthesis requires approximately 20 genes and over 30 enzymatic steps, depending on the anaerobic or aerobic pathway and diversity of the genetic pathway (Raux et al., 2000). Additional genes are involved in $\mathrm{B}_{12}$-transport and $\mathrm{B}_{12}$-dependent enzymes. Furthermore, $\mathrm{B}_{12}$ is exclusively synthesized by select bacteria and archaea, at a substantial metabolic cost (Roth et al., 1996).

Based on genomic analyses, cobalamin biosynthesis in the ocean is carried out by three groups: heterotrophic bacteria, chemoautotrophic Thaumarchaeota, and phototrophic cyanobacteria (Heal et al., 2017). Prochlorococcus, the most abundant photosynthetic organisms on Earth with a highly streamlined genome, synthesizes pseudocobalamin $\left(\mathrm{pB}_{12}\right)$ along with its cyanobacterial relatives. $\mathrm{pB}_{12}$ uses adenine for the lower ligand compared to DM (see Fig.1-1) and is not bioactive in eukaryotic algae without molecular remodeling (Heal et al., 2017). Deeper in the water column, Thaumarchaeota are the dominant producers of $\mathrm{B}_{12}$ (Doxey et al., 2015). The ultimate source of $\mathrm{B}_{12}$ to euphotic zone seawater is de novo synthesis by bacterioplankton. However, in Antarctic waters, without cyanobacteria, bacterial and archaeal $\mathrm{B}_{12}$ production likely play a more important role (Doxey et al., 2015).

The oceanographic abundance of $\mathrm{B}_{12}$ and $\mathrm{Co}$ in seawater is the result of environmental sources and sinks, with surface ocean rates largely driven by marine microbial activity. The related $\mathrm{Co}$ and $\mathrm{B}_{12}$ cycles in Antarctic systems is briefly summarized here and reviewed in depth in Chapter 3, Appendix 3.A. Cobalt is the least abundant inorganic nutrient in the ocean, as a result of low input fluxes and 

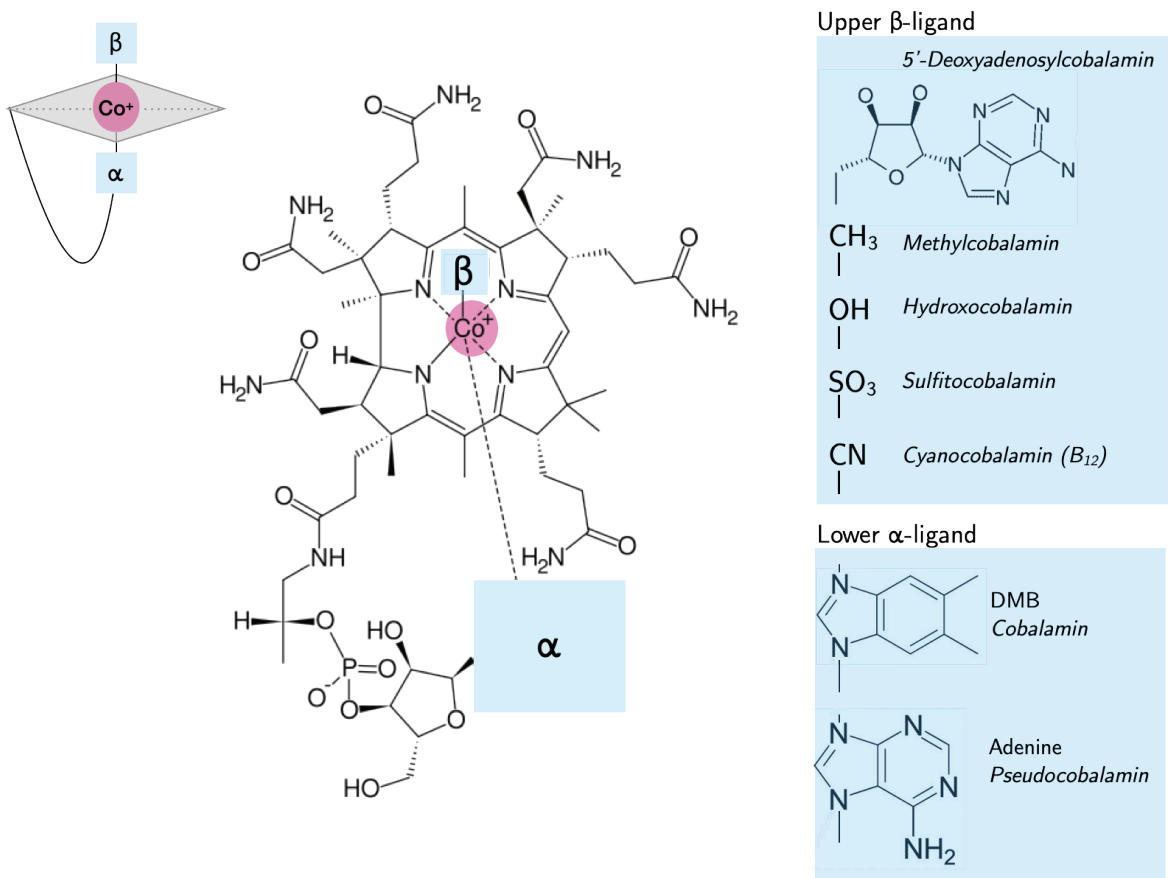

Figure 1-1: The structure of cobalamin consits of a central corrin ring and Co atom center, with variable upper and lower ligands. Cyanocobalamin (commonly referred to as vitamin $\mathrm{B}_{12}, \mathrm{~B}_{12}$, or simply cobalamin) has a -CN upper $\beta$-ligand and DMB lower $\alpha$-ligand. The $\beta$-ligands are interchangable and can be modified for specific biochemical reactions. The $\alpha$-ligand distinguishes cobalamain (DMB) and pseudocobalamin (Adenine), the latter is synthesized by cyanobacteria. The figure is modified from Watanabe (2007).

its removal by biological uptake, chemical scavenging, and photo-degradation. In most oceanographic regions Co concentration has a hybrid-type profile, reflecting uptake as a nutrient in the euphotic zone, accumulation at the base of the euphotic zone due to remineralization, and a decreasing amount with depth due to scavenging via co-oxidation with manganese-oxidizing bacteria. Whereas $\mathrm{B}_{12}$ has a consistent nutrient-like profile, being consumed in the sunlit ocean and accumulating or constant with depth. This combination of factors increase the potential for $\mathrm{B}_{12}$-limitation in Antarctic waters, including: reduced $\mathrm{B}_{12}$ production as a result of no cyanobacteria and low heterotrophic bacterial densities, high photodegradation due to high UVradiation, low atmospheric inputs due to ice cover and low continental dust, and the heterogeneous availability due to varied sea-ice environments.

Since an early stage, both $\mathrm{Co}$ and $\mathrm{B}_{12}$ have been "institutionalized" or "locked in" to the course of evolution, leaving many auxotrophs dependent on $\mathrm{B}_{12}$-producers (Eschenmoser, 1988). But, life has also evolved a range of metabolic strategies to cope with the scarce and variable biologically-derived $\mathrm{B}_{12}$ supply. Multiple coexisting $\mathrm{B}_{12}$-strategies exist in modern lifeforms to take advantage of $\mathrm{B}_{12}$ or escape this vitamin requirement entirely. The main use of $\mathrm{B}_{12}$ in cells is for methionine synthase, and $\mathrm{B}_{12}$-auxotrophy primarily stems from which methionine synthase isoform(s) (MetE or MetH) an organism encodes in its genome. 
To deal with the scant, variable environmental supply of $\mathrm{B}_{12}$, eukaryotic algae have three potential strategies: 1) escape $\mathrm{B}_{12}$-requirements by only having MetE, 2 ) be $\mathrm{B}_{12}$-dependent by only having $\mathrm{MetH}$, or 3 ) be flexible by maintaining both MetE and MetH isoforms. Most microalgae only have MetH and are thus $\mathrm{B}_{12}$-auxotrophs, rendered completely dependent on a local exogenous/environmental supply of $\mathrm{B}_{12}$ from associated or neighboring bacterioplankton (Croft et al., 2005, 2006). Some microalgae are flexible and facultatively inter-replace MetH and MetE in response to $\mathrm{B}_{12}$ availability, as observed in P. tricornutum, and C. reinhardtii (Bertrand et al., 2013; Bunbury et al., 2020). No microalgae are $B_{12}$-independent. Though all land plants and fungi are because they only contain MetE and $\mathrm{B}_{12}$-independent proteins. Organisms that have both MetE and MetH have a flexible ecophysiology to $\mathrm{B}_{12}$ availability, but at the added costs of maintaining and regulating MetE protein synthesis (Aretakis et al., 2019). In highly seasonal Southern Ocean environments, with variable bacterioplankton $\mathrm{B}_{12}$ production and environmental conditions, having a $\mathrm{B}_{12}$-flexible ecophysiology could infer an important competitive advantage. This curious split between marine and land plants and the inter-domain metabolic dependence between marine eukaryotic algae and bacterioplankton, highlights the fact that the biogeochemistry and ecological interactions underlying $\mathrm{B}_{12}$-cycling in the ocean are of fundamental importance to marine microbial ecosystems.

Since the early days of algal culturing, it has been known that most algae require a culture medium supplemented with trace metals like iron ( $\mathrm{Fe})$ and cobalt ( $\mathrm{Co})$ and organic micronutrients like cobalamin $\left(\mathrm{B}_{12}\right)$ in order to grow (Droop, 1957). Over half of eukaryotic algae and approximately one third of bacteria are $\mathrm{B}_{12}$-auxotrophs, indicating that bacterioplankton are also major $\mathrm{B}_{12}$ consumers in the ocean (Croft et al., 2005; Ho et al., 2003). A literature survey and culture experiment of multiple algal lineages by Croft et al. (2005) found that dinophyta, haptophyta, heterokontophyta, and chlorophyta lineages had a higher proportion of species deemed to be $\mathrm{B}_{12}$-dependent, and coccolithophorids and dinoflagellates have high cellular quota and requirements for Co (Ho et al., 2003). Cyanobacteria have a higher Co requirement than eukaryotic phytoplankton, which is expected due to their $\mathrm{pB}_{12}$ synthesis (Saito et al., 2002; Sunda and Huntsman, 1995).

The genetic basis for $\mathrm{B}_{12}$ auxotrophy is due to the loss of the $\mathrm{B}_{12}$-independent isoform of methionine synthase (MetE), which has been lost multiple, independent times throughout evolutionary history in many different environments, suggesting a consistent ambient supply of $\mathrm{B}_{12}$ (Helliwell et al., 2011). An important note is that while auxotrophic eukaryotic algae have lost the ability for de novo synthesis vitamins $\mathrm{B}_{1}$ (thaimine) and $\mathrm{B}_{7}$ (biotin), $\mathrm{B}_{12}$-auxotrophy is the result of the loss of meteE gene (Croft et al., 2006; Helliwell et al., 2013). An experimental evolution experiment in $C$. reinhardtii demonstrated that after 500 generations in $\mathrm{B}_{12}$-replete media, the culture lost its functional copy of meteE, rendering it a $\mathrm{B}_{12}$-auxotroph (Helliwell et al., 2015). To overcome $B_{12}$-limitation, auxotrophs invest in uptake mechanisms, symbiotic relationships, or the ability to salvage $\mathrm{B}_{12}$ components from the environment.

Vitamin salvaging is an adaptation that allows microbes to overcome $\mathrm{B}_{12}$ limitation by assimilating $B_{12}$ vitamers or degradation products and converting them into bioactive forms. Both auxotrophic microalgae and bacterioplankton have acquired the 
genes in the latter steps of $\mathrm{B}_{12}$ biosynthesis, which infer the genetic potential to remodel cobalamin compounds, via horizontal gene transfer (HGT) from prokaryotes that (Heal et al., 2017; Swithers et al., 2012; Vancaester et al., 2020). A comparative genomics analysis of 11,000 bacteria species found that while a large majority ( $86 \%$ ) had $\mathrm{B}_{12}$-dependent enzymes, only a fraction $(37 \%)$ were predicted to have the full biosynthetic pathway, and a smaller percentage (17\%) had the end of the pathway to salvage $\mathrm{B}_{12}$ (Shelton et al., 2019). Due to the persistence of $\mathrm{B}_{12}$ throughout evolution, the vitamin has a range of uses in prokaryotes and fewer, though significant uses, in eukaryotes; however, its scarcity has necessitated $\mathrm{B}_{12}$-independent alternatives that also come at a fitness cost. For $\mathrm{B}_{12}$-auxotrophs, salvaging cobalamin is a solution that allows for $\mathrm{B}_{12}$-dependent enzymes with only a partial cost of biosynthesis.

The general finding that cobalamin uptake, use, and salvage is more widespread than cobalamin synthesis, suggests that distributing the large cost of $\mathrm{B}_{12}$ production to a subset of species is metabolically beneficial from a community- or ecosystemperspective (Giovannoni, 2012). The Black Queen Hypothesis presented by Morris et al. (2012) provides a compelling framework in which to discuss the fitness benefits and costs of various $B_{12}$-strategies and is nicely reviewed in Giovannoni (2012). Briefly, the hypothesis posits that nutritional dependencies arise as a result of adaptive gene loss when the vitamin is consistently produced and available due to a microbial community member. Interestingly, the more energetically expensive and/or rare a metabolite, the more likely it is to enable a stable and mutually-beneficial cross-feeding relationship between auxotrophs. This was demonstrated in a pairwise co-culture of E. coli amino acid auxotrophs in a beautiful study by Wintermute and Silver (2010), Mee et al. (2014) and later in Sacchromyces cerevisiae by Shou et al. (2007). As detailed, $\mathrm{B}_{12}$ is an expensive metabolite that is synthesized by only some prokaryotes in the ocean, providing an essential function for some populations and ultimately benefitting the overall community. These nutrient exchange networks highlight the need to examine microbes from a systems perspective and understand the individual roles within a larger distributed metabolism at a community level.

As an aside, it is important to note that interspecies interactions involving the exchange of $\mathrm{B}_{12}$ are not limited to marine systems, highlighting a widespread phenomenon in nature for nutrient cross-feeding. The human gut microbiome has bacteria that synthesize and salvage $B_{12}$ in the large intestine, yet in humans $B_{12}$ can only be absorbed in the small intestine. This revealed that corrinoid compounds affect the gut microbial ecology, "separate" from the hosts' nutritional needs, but with possible feedbacks on host health (Degnan et al., 2014). Recent comparative 'omics into the microbiome of lichen Lobaria pulmonaria found that bacteria are also a key part of the symbiosis between algae and fungi, and supply $\mathrm{B}_{12}$ to algae, along with growth hormones, pathogen defense, and nutrients including nitrogen, phosphorous, and sulfur (Grube et al., 2015). Lichens are varied, ubiquitous, and an ancient symbiotic partnership between algae, fungi, and bacteria. High levels of $\mathrm{B}_{12}$ are found in the dense phytoplankton growth on the underside of sea ice, produced by bacteria and cycled among algae (Taylor and Sullivan, 2008). Cicadas depend on symbiotic bacteria for essential vitamins and amino acids, and indirectly depends on $\mathrm{B}_{12}$ due to its auxotrophic symbiont $H$. cicadicola that synthesized $\mathrm{B}_{12}$ and only has 
MetH (McCutcheon et al., 2009). In the ocean, in situ nutrient dependencies are challenging to study, but lab-based co-cultures have revealed symbiotic relationships between $\mathrm{B}_{12}$-producing bacteria and photosynthate-providing algae could produce a stable coexistence (Croft et al., 2005; Grant et al., 2014; Helliwell et al., 2014; Kazamia et al., 2012). A recent study even confirmed that land plants, which do not need $\mathrm{B}_{12}$ for their metabolisms, uptake $\mathrm{B}_{12}$ and store it in vaculoues, with a possibility for release into soils, which could affect their root microbiomes (Lawrence et al., 2018). These examples support the notion that expensive metabolites form the foundation of stable co-existence between metabolically-dependent partners (Wintermute and Silver, 2010).

However, while nutritional auxotrophies may shape microbial interactions in a community, the environment always selects. In ever-changing environments like the ocean, ecosystem conditions determine whether or not an organisms' $\mathrm{B}_{12}$-strategy has a higher fitness benefit or cost. In microalgae, the distribution of $\mathrm{B}_{12}$-auxotrophs across species has no phylogenetic pattern, suggesting instead that environmental conditions select for the $\mathrm{B}_{12}$-strategy. A prime example is that diatoms in the Southern Ocean more often contain MetE and MetH, relative to diatoms from other oceanographic regions that are more likely to contain just MetH (Ellis et al., 2017; Vancaester et al., 2020).

The Southern Ocean has a unique set of ecological and environmental conditions that preclude it to $\mathrm{B}_{12}$-limitation. In the study of $\mathrm{Co}$ and $\mathrm{B}_{12}$ biogeochemistry, Antarctic waters are considered an end member due to their extreme conditions and seasonal dynamics (Saito et al., 2010). A specific combination of factors increases $\mathrm{B}_{12^{-}}$ limitation potential in Antarctic waters, including: reduced $\mathrm{B}_{12}$ production as a result of no cyanobacteria and low heterotrophic bacterial densities, high photodegradation due to high UV-radiation (approximate half life of $\mathrm{B}_{12}$ is 4 days in the surface ocean), low Co inputs due to ice cover and low atmospheric dust inputs, the heterogeneous spatial availability due to varied sea-ice environments, and a highly variable, seasonal $\mathrm{B}_{12}$ production by bacterioplankton due to ecological dynamics. The related Co and $\mathrm{B}_{12}$ cycles in Antarctic systems are reviewed in depth in Chapter 3 and Appendix 3.A. The same conditions that make the Southern Ocean susceptible to $\mathrm{B}_{12}$-limitation, can also provide an advantage to some community members. Bacterioplankton may be relieved of cobalt limitation, due to a lack competition from cyanobacteria. This would be reflected in comparatively low Co uptake rates compared to other oceanographic regions. Cobalt limitation can limit $\mathrm{B}_{12}$ production, as observed in the North Atlantic Ocean (Panzeca et al., 2008).

Over the past thirty years of nutrient amendment experiments in the Southern Ocean, scientists have determined that these systems are primarily limited by iron (Fe) and co-limited at times by $\mathrm{B}_{12}$ (Bertrand et al., 2013; Martin, 1990; Moore et al., 2013; Panzeca et al., 2006; Sedwick et al., 2000). Iron was found to be limiting to both $P$. antarctica and diatoms at concentrations of $<0.2 \mathrm{nM}$, but $P$. antarctica had sufficient iron at $1 \mathrm{nM}$ (Sedwick et al., 2000). Along the Antarctic Peninsula, was the first report of Fe- $\mathrm{B}_{12}$ co-limitation, followed by a similar results in the Ross Sea in soon after (Bertrand et al., 2007, 2011b; Panzeca et al., 2006). In the Ross Sea, P. antarctica was limited by iron, whereas the addition of $\mathrm{Fe}$ and $\mathrm{B}_{12}$ stimulated diatom 
growth, specifically the species Pseudonitzschia subcurvata (Bertrand et al., 2007). In McMurdo Sound, a diatom-dominated community at the sea ice edge was limited by both $\mathrm{Fe}$ and $\mathrm{B}_{12}$ (Bertrand et al., 2015). One may wonder why be a $\mathrm{B}_{12}$-auxotroph in Antarctic waters at all? Diatoms are a dominant bloom-forming species in this region, both in sea ice and in seawater. Diatoms are considered to be opportunists, with some of the highest growth rates among phytoplankton. Maintaining $\mathrm{B}_{12}$-dependent metabolic pathways infers a fitness benefit an can enable increased growth rate, under conditions of sufficient/replete $\mathrm{B}_{12}$ and other micronutrients like Fe. To date, the $\mathrm{B}_{12}$ ecophysiology of polar diatoms is better studied compared to other important regional phytoplankton that also likely require $\mathrm{B}_{12}$ (Croft et al., 2006), including dinoflagellates, cryptophytes, and the bloom-forming colonial haptophyte Phaeocystis antarctica.

Our understanding of $\mathrm{B}_{12}$ dynamics in the ocean has progressed over the past decade, but several challenges remain. A major challenge is measuring the often picomolar or subpicomolar concentrations of $\mathrm{B}_{12}$ in seawater. Advances in mass spectrometry methods combined with solid phase extraction and liquid chromatography have enabled the detection of $\mathrm{B}_{12}$ and its vitamers at natural concentrations (Gómez-Consarnau et al., 2018; Heal et al., 2014; Suffridge et al., 2017). However, the standing stock of the vitamin is the remainder after it has been assimilated by microbes. A major outstanding question is, what is the flux of $\mathrm{B}_{12}$ into biomass? Furthermore, what are the ecological or environmental conditions that drive $\mathrm{B}_{12}$ demand and uptake? In order to mechanistically understand the demand, we must examine what are the nutritional needs and resulting $\mathrm{B}_{12}$-ecophysiologies of keystone phytoplankton and bacterioplankton. Finally, for Antarctic systems, how much of community production and assembly dynamics are governed by micronutrients, specifically $\mathrm{Fe}, \mathrm{Co}$, and $\mathrm{B}_{12}$ ? A holistic understanding of the biogeochemistry of $\mathrm{B}_{12}$ and its climate consequences requires an integrated approach across spatial, temporal, and biological scales to connect the metabolisms of individual species with the network of organic nutrient exchange in the environmental context that determines the relative fitness of marine microbes.

\subsection{Thesis Overview}

Given the potential for this region to be co-limited by $\mathrm{Fe}$ and $\mathrm{B}_{12}$, it is important to understand the economy of $\mathrm{B}_{12}$ between bloom-forming phytoplankton and bacterioplankton in Antarctic waters. Studying the underlying micronutrient limitation of Antarctic phytoplankton communities is especially important now, as anthropogenic climate change accelerates the melt rates of glaciers and sea ice, which could fundamentally alter the micronutrient balance, ecosystem structure, and potential for carbon export. $\mathrm{B}_{12}$ is an essential part of microbial ecology in marine systems. Despite the important role of nutrient cycling of trace organics among marine microbes, these metabolites are not yet integrated into global ocean ecosystem models. This thesis seeks to address that gap by using $\mathrm{B}_{12}$ as a model metabolite to examine the dynamics and consequences of nutrient exchange in natural seawater communities.

The overarching goal of this thesis is to contribute to the mechanistic understanding 
of the Antarctic $\mathrm{B}_{12}$ biogeochemical cycle using targeted measurements in laboratory and field experiments and the development of a model with which to examine $\mathrm{B}_{12^{-}}$ driven ecosystem dynamics. This thesis was designed to examine algal-bacterial $\mathrm{B}_{12}$ cycling from multiple spatial-temporal perspectives (summarized in Fig.1-2) with the aim of gaining a more holistic understanding. The motivation and research strategy are summarized in this section.

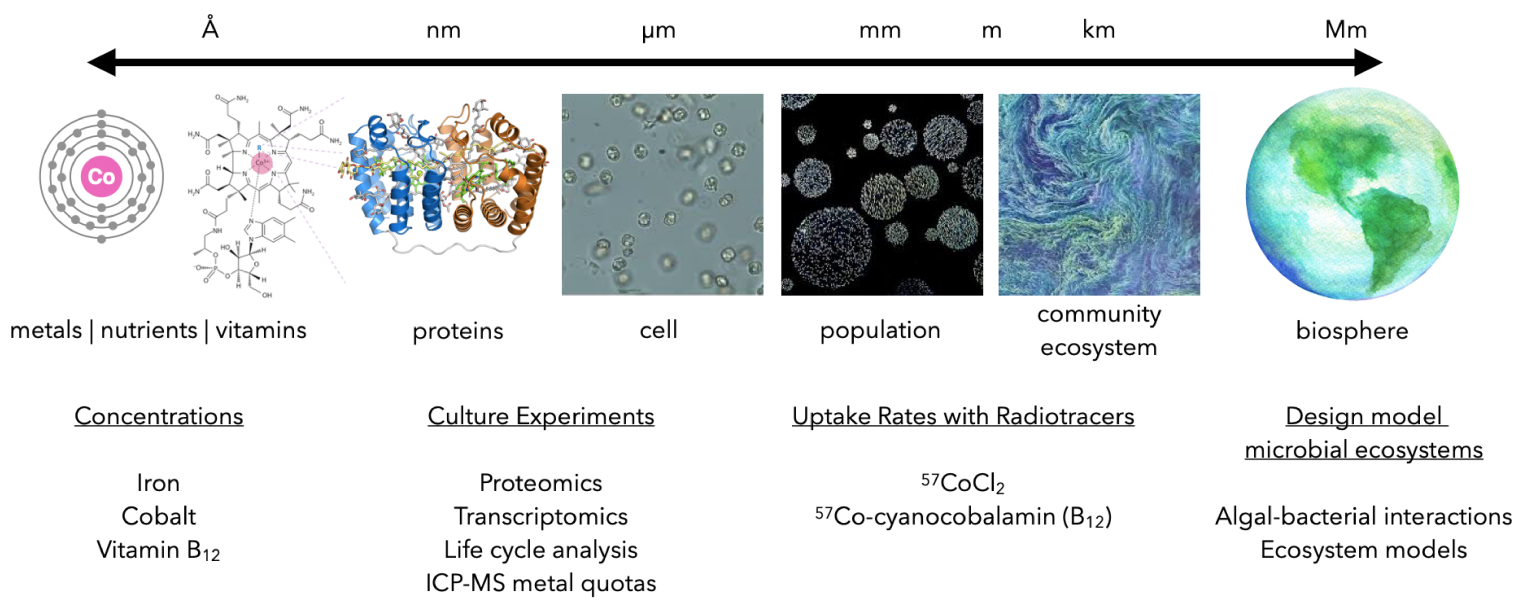

Figure 1-2: This thesis aims to take a holistic approach to the study of $\mathrm{B}_{12}$ cycling in Antarctic coastal ecosystems by marine microbes. This complex system is multi-scale in nature and requires a multi-scale approach. By using methods that cut across spatial, temporal, and biological scales, the discrete analyses can be combined into a more continuous understanding of the complex system. The various scales examined in this work are presented along with the methods used at those scales.

\section{Lab Component: $\mathrm{B}_{12}$ ecophysiology of $P$. antarctica}

P. antarctica is a keystone Antarctic phytoplankton that forms massive blooms in its colonial life stage. This region is demonstrated to be co-limited by iron $(\mathrm{Fe})$ and $\mathrm{B}_{12}$, yet this important species' $\mathrm{B}_{12}$ needs were not examined through laboratory studies. I grew $P$. antarctica cultures across a wide range of $\mathrm{B}_{12}$ conditions found in the ocean, and measured its metabolic response through life cycle analysis, proteomics, transcriptomics, and physiological observations. The $\mathrm{B}_{12}$ dose-response experiment was conducted at low and high iron concentrations, that were previously demonstrated to engender the solitary or colonial morphotype, respectively (Bender et al., 2018). This multi-factor micronutrient limitation experiment resulted in a matrix of observations that enable the comparison of not only $\mathrm{B}_{12}$-ecophysiology under different iron conditions, but also a comparison across the dominant life cycle stages. We discovered a putative MetE in $P$. antarctica, which was previously thought to only contain MetH, and furthermore show that this protein sequence is present in a range of other polymorphic, flagellated microalgae and other prokaryotes as well. We identify proteins that significantly respond to $\mathrm{B}_{12}$ and demonstrate that $P$. antarctica has a flexible $\mathrm{B}_{12}$ ecophysiology. 


\section{Field Component: Co and $\mathrm{B}_{12}$ uptake in natural seawater communities}

Both $\mathrm{Co}$ and $\mathrm{B}_{12}$ are scarce and rapidly cycled in the surface ocean. In order to better constrain the flux of these micronutrients into the marine microbial community in Antarctic coastal water environments, in situ uptake rates were measured using the radiotracer incubation method with ${ }^{57} \mathrm{Co}$. The spatial and depth coverage, along with the size-fractionated analysis, makes this dataset the largest of its kind. This data, integrated with hydrographic and phytoplankton chemotaxonomic data enables the exploration of potential drivers of $\mathrm{B}_{12}$ and Co uptake. The highest uptake rates were measured in the surface ocean $(\geq 100 \mathrm{~m})$ in regions of fresher, warmer waters. Both ${ }^{57} \mathrm{Co}$ and ${ }^{57} \mathrm{~B}_{12}$ uptake were significantly correlated with diatom indicator pigments and biogenic silica. Regional differences were found between uptake patterns in the Amundsen Sea, Ross Sea, and Terra Nova Bay, corresponding to the hydrographic conditions and local marine microbial communities. Interested readers are directed to Appendices 3.A and 3.B for a review of the ecological Co and $\mathrm{B}_{12}$ cycles in Antarctic coastal environments and a water mass characterization of continental slope waters sampled during the CICLOPS research expedition for the analysis in Chapter 2.

\section{Model Component: Micronutrient drivers of seasonal succession in Antarctic marine ecosystems}

Prior studies have posited that the microbial community succession pattern is due to seasonal changes in irradiance, mixed layer depth, and iron (Arrigo et al., 1999; Petrou et al., 2016). An alternative hypothesis to the existing paradigm of coastal Antarctic marine microbial community succession is developed based on the findings in Chapters 2 and 3. This novel hypothesis is described and tested in a numerical ecosystem model model in Chapter 4. Using this model, the expected seasonal succession pattern emerges without invoking mixed layer depth or light levels, as other hypotheses have suggested. This hypothesis proposes that micronutrient supplies of Fe and $\mathrm{B}_{12}$ drive the observed seasonal succession from $P$. antarctica solitary cells to colonies, bacterioplankton that grow on dissolved organic carbon from community detritus, and then $\mathrm{B}_{12}$-auxotrophic diatoms. A P. antarctica functional type is modeled based on an Fe-dependent transition between morphotypes and a diffusive boundary layer that affects nutrient uptake, especially of large colonies composed of many cells (Bender et al., 2018; Ploug et al., 1999). Though relatively simple, this model demonstrates how co-existence is possible due to phytoplankton and bacterioplankton model types' growth being limited by distinct micronutrients and that a system of cross-feeding interactions of resources can result in the seasonal succession pattern observed in the coastal Antarctic region.

In the remainder of this dissertation, I elucidate some of the ecological drivers of cobalamin cycling in Antarctic marine ecosystems. Chapter 2 examines the iron and 
$\mathrm{B}_{12}$ ecophysiology of $P$. antarctica morphotypes using transcriptomics and proteomics approaches and show that this species is not auxotrophic for $\mathrm{B}_{12}$. Chapter 3 reports the largest set of measured uptake rates of $\mathrm{Co}$ and $\mathrm{B}_{12}$ conducted in Antarctic seas using radiotracer incubations, and finds that hydrographic and ecological conditions that support a pattern of diatom-driven, community-level uptake rates. Chapter 4 combines the insights of the first two chapters to inform the development of a numerical model of micronutrient-driven seasonal succession in Antarctic marine microbial communities. Taken together, the results of these studies provide a novel perspective on $\mathrm{B}_{12}$-limitation and $\mathrm{B}_{12}$-cycling in Antarctic marine microbial communities with implications for regional biogeochemical cycling and feedbacks on global climate. 


\section{Chapter 2}

\section{Phaeocystis antarctica has a flexible vitamin $\mathrm{B}_{12}$ ecophysiology: A multi-omic study reveals its response to iron and cobalamin stress}

"... it became clear to me that plankton ecologists fall out into two groups: Those who delight in finding the patterns in nature that can be explained by size, and those who delight in finding exceptions to the established sizedependent rules... The simplicity of the general relationships serves as a stable backdrop against which the exceptions can shine. By understanding the forces that have driven the design of these exceptions, we can begin to understand the ecology that has shaped past and present planktonic ecosystems." - Sallie W. Chisholm (1992).

Context: The data presented in this chapter was produced during my time as a guest student in the Saito Lab at WHOI. I first thank Mak Saito and Mick Follows for the opportunity to pursue this collaboration through which I was able to use trace metal clean methods, algal culturing techniques, cutting edge proteomics methods, and bioinformatics analyses to examine the iron and $\mathrm{B}_{12}$ metabolism of $P$. antarctica. I thank Dawn Moran for invaluable guidance and assistance in growing $P$. antarctica cultures under trace metal clean conditions; Matt McIlvan for his expertise in mass spectrometry methods and performing shot-gun and targeted proteomics; Andy Allen and Zoltan Fussy for processing the transcriptomic data, developing a transcriptomic assembly, guidance on transcriptomic analysis, collaboration in constructing the phylogenetic tree of a putative protein identified in this study, and providing the initial algal cultures. The phylogenetic methods were provided by Z. Fussy. I also thank Natalie Cohen and Arianna Krinos for assistance in bioinformatics methods used throughout this study. 


\section{Abstract}

Phaeocystis antarctica is a keystone phytoplankton species in coastal Antarctic marine microbial ecosystems where it dominates early-season blooms. In this oceanographic region, phytoplankton growth is primarily limited by iron and can be co-limited by cobalamin (vitamin $\mathrm{B}_{12}$ ). While several studies have examined the iron metabolism and colony formation of $P$. antarctica, its ecophysiology in response to $\mathrm{B}_{12}$ has not been experimentally explored. We present the results of a laboratory culture study and complementary transcriptomic and proteomic analyses of its $\mathrm{B}_{12}$-responsive metabolism. Predominantly solitary and colonial cultures were grown under low and high iron conditions, respectively, and over a wide range of $\mathrm{B}_{12}$ concentrations. Proteomic and transcriptomic analyses revealed that $P$. antarctica maintains both methionine synthase isoforms - $\mathrm{B}_{12}$-dependent MetH and a putative $\mathrm{B}_{12}$-independent MetE-and inter-replaces them in response to cobalamin availability. Furthermore, numerous proteins were significantly differentially abundant in response to limited or replete $\mathrm{B}_{12}$, including proteins involved in methionine cycling, methyltransferase reactions, and cell cycle regulation. A previously unrecognized MetE protein sequence was identified via proteomics and an in silico construct was found in the transcriptomic assembly. A phylogenetic analysis of this putative metE gene revealed homologues in heterotrophic bacterioplankton and eukaryotic algae, including three $P$. antarctica strains, other haptophytes like Emiliania huxleyi, and a range of protists that have flagella and polymorphic life cycles. The phylogenetic pattern of this putative MetE implies a potential metabolic connection to flagella and cell cycle regulation. This study confirms that Phaeocystis antarctica strain CCMP 1871 is not auxotrophic for $\mathrm{B}_{12}$. Indeed, this $P$. antarctica strain has the metabolic capability to grow in the absence of and in low concentrations of $\mathrm{B}_{12}$. Instead, it has a flexible $\mathrm{B}_{12}$-ecophysiology, which is advantageous in the seasonally variable Antarctic waters that are prone to $\mathrm{B}_{12^{-}}$ limitation. These results have important implications for the micronutrient limitation of natural $P$. antarctica populations, patterns of seasonal succession, and ecosystem dynamics in Antarctic marine microbial communities.

\subsection{Introduction}

The coastal Southern Ocean is a region of seasonally high primary productivity that impacts global climate. Phytoplankton blooms develop in the coastal Antarctic polynyas, large expanses of open water surrounded by sea ice, which emerge with the onset of austral spring. During this time, increased sunshine, iron availability, and warmer temperatures create favorable growth conditions in the typically highnutrient, low-chlorophyll waters. It is well documented that Antarctic marine microbial communities are primarily limited (and thus driven) by light and iron-but at times can be co-limited for the organic nutrient cobalamin (vitamin $\mathrm{B}_{12}$ ) (Arrigo et al., 2012; Bertrand et al., 2007, 2011a,b, 2015; Martin, 1990; Sedwick et al., 2000). Cobalamin is a biologically-derived organic nutrient that is only produced by some bacteria and archaea, but is an essential nutrient for most eukaryotic microalgae. Indeed, nearly 
half of all surveyed marine protists are $\mathrm{B}_{12}$-auxotrophs, which require the vitamin but are unable to synthesize it de novo. Consequently, many algae depend on a consistent $\mathrm{B}_{12}$ supply from local bacterioplankton, which in turn depend on phytoplankton for a supply of dissolved organic carbon compounds (Croft et al., 2006). Furthermore, the standing stock of $\mathrm{B}_{12}$ in seawater is scant and variable, ranging on the order of $<1 \mathrm{pM}$ to $10 \mathrm{pM}$ (Panzeca et al., 2009). Given the varied cobalamin needs of marine phytoplankton, vitamin $\mathrm{B}_{12}$ has the potential to limit primary productivity, alter microbial community structure, and influence regional biogeochemistry with important climate feedbacks.

In this study, we investigate the $\mathrm{B}_{12}$ requirements of Phaeocystis antarctica, a keystone phytoplankton species that forms massive, sustained blooms in the Antarctic Southern Ocean. Here, P. antarctica grows in near monospecific blooms on the order of $10^{7}$ cells $\mathrm{L}^{-1}$ that can last through the austral summer (Schoemann et al., 2005). P. antarctica blooms directly impact regional marine microbial ecology, broader ecosystem dynamics, the biogeochemical cycling of carbon, nutrient, and sulfur cycles, and the potential carbon export to the deep ocean. The highly-productive $P$. antarctica cells can quickly draw down $\mathrm{CO}_{2}$ concentrations to below $100 \mathrm{ppm}$ through their collective photosynthesis and have the potential to assimilate twice as much $\mathrm{CO}_{2}$ per mole of phosphate from seawater as diatoms (Arrigo et al., 1999, 2003; Schoemann et al., 2005; Smith et al., 2003; Yager et al., 2016). This productivity is a source of organic carbon and nutrients to the marine microbial food web, and consequently has the potential to affect the growth and succession of bacterioplankton and other phytoplankton species (Ducklow et al., 2001; Kirchman et al., 2000; Rousseau et al., 2007). P. antarctica, like other haptophyte algae, is an important regional producer of dimethylsulfoniopropionate (DMSP), which has several uses in algal cells, including cryoprotectant, osmolyte, and antioxidant (DiTullio et al., 2000). Importantly, DMSP is the precursor of dimethyl sulfide (DMS), a climatically-active gas crucial to the sulfur biogeochemical cycle and a contributor to cloud nucleation. The micronutrient controls on $P$. antarctica bloom dynamics therefore have regional and global implications for carbon and nutrient cycling and climate.

Given the important role of $P$. antarctica in Antarctic marine microbial ecology and biogeochemistry, examining how $\mathrm{B}_{12}$ influences its growth (as single cell and colonial morphotypes) is necessary to understanding the $\mathrm{B}_{12}$-driven community dynamics in Antarctic marine microbial ecosystems. The development of $P$. antarctica blooms is primarily a life cycle event. $P$. antarctica has a polymorphic life cycle that alternates between free-living solitary, flagellated cells and colonial cells living on a mucilaginous matrix. Free-living single cells are motile nanoplankton of $2-6 \mu \mathrm{m}$ in diameter that are propelled by two flagella and one haptonema. These cells metamorphose into larger, nonflagellated colonial cells of $4-6 \mu \mathrm{m}$ in diameter. These colonial cells are uniformly distributed in the inner periphery within the colony matrix, positioned about one cell width from the external "skin" of the colony (Zingone et al., 2011). P. antarctica can grow as a continuum of colony sizes, with diameters spanning the micron-scale up to visible millimeter-scale colonies (Schoemann et al., 2005).

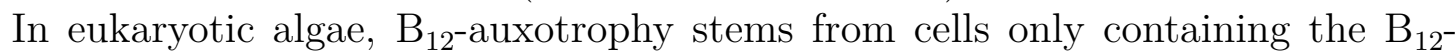
dependent isoform of methionine synthase $(\mathrm{MetH})$; the other isoform is $\mathrm{B}_{12}$-independent 
methoinins synthase (MetE) (Croft et al., 2005). Cobalamin is thought to be directly used in a few key enzymes: $\mathrm{B}_{12}$-dependent methionine synthase $(\mathrm{MetH})$, class II ribonucleotide reductase (RNR II), and methylmalonyl-CoA-mutase (MMCM). Both protein isoforms of methionine synthase are metalloenzymes that catalyze the conversion of 5-methyltetrahydrofolate and homocysteine into tetrahydrofolate and methionine, respectively, which thereby links methionine and folate cycles. MetE and MetH are not homologous and though they achieve the same function, MetE has a much lower catalytic efficiency, by 100-fold (Bertrand et al., 2013; Gonzalez et al., 1992). For algae, methionine is an essential amino acid for protein synthesis, the precursor to S-adenosylmethionine (SAM/AdoMet) - a key methylating agent/methyl donor and radical source involved in DNA methylation, vitamin $\mathrm{B}_{1}$ (thiamine) synthesis, and DMSP biosynthesis. Methionine synthase is important for one-carbon metabolism and folate recycling and $\mathrm{B}_{12}$ is also indirectly involved in a range of cellular functions pertaining to protein, RNA, and DNA synthesis and regulation (Bertrand et al., 2011a, 2013; Romine et al., 2017).

To deal with the scant, variable environmental supply of $\mathrm{B}_{12}$, eukaryotic algae have three potential strategies. Most microalgae only have MetH and are thus $\mathrm{B}_{12^{-}}$ auxotrophs, rendered completely dependent on a local exogenous/environmental supply of $\mathrm{B}_{12}$ from associated or neighboring bacterioplankton (Croft et al., 2005, 2006). Some microbes are flexible and inter-replace MetH and MetE in response to $\mathrm{B}_{12}$ availability, as observed in P. tricornutum, and C. reinhardtii (Bertrand et al., 2013; Bunbury et al., 2020). No microalgae are completely $B_{12}$-independent (there is one known exception of a red algal species), though all land plants and fungi only contain MetE and $\mathrm{B}_{12}$-independent proteins. Whether or not an algal species maintains MetE has no phylogenetic pattern, and is likely a result of environmental selection and $B_{12}$ availability (Helliwell et al., 2011).

Recent studies support the notion of biogeographical selection pressures to maintain MetE, as indicated by a significantly larger proportion of Southern Ocean diatoms having both methionine synthase isoforms relative to other oceanographic regions (Ellis et al., 2017). Organisms that have both MetE and MetH have a flexible ecophysiology to $\mathrm{B}_{12}$ availability, but at the added costs of maintaining and regulating MetE protein synthesis (Aretakis et al., 2019). In highly seasonal Southern Ocean environments, with variable bacterioplankton $\mathrm{B}_{12}$ production and environmental conditions, having a $\mathrm{B}_{12}$-flexible ecophysiology could infer an important competitive advantage. This region has relatively higher cobalt availability, suggesting that the trace metal is likely not limiting to $\mathrm{B}_{12}$ synthesis (Saito et al., 2002). This curious split between marine and land plants and the inter-domain metabolic dependence between marine eukaryotic algae and bacterioplankton, highlight the fact that the biogeochemistry and ecological interactions underlying $\mathrm{B}_{12}$-cycling in the ocean are of fundamental importance to marine microbial ecosystems.

What role does $\mathrm{B}_{12}$ have in the ecology of $P$. antarctica blooms in Antarctic waters? Prior to this study, the ecophysiology of $P$. antarctica in response to $\mathrm{B}_{12}$ limitation had not been examined in culture. Recent micronutrient studies focused on the iron-responsive metabolism in P. antarctica strains CCMP 1871 and CCMP 1374 (Bender et al., 2018), and manganese and iron limitation in strain CCMP 1374 (Wu 
et al., 2019), Zn-Co co-limitation (Saito and Goepfert, 2008), and acclimiation to light (Sedwick and DiTullio, 1997; Sedwick et al., 2000). A recent study using bioinformatic analyses reported that no haptophyte maintains both MetE and MetH, and that there is widespread $\mathrm{B}_{12}$-auxotrophy among this lineage (Nef et al., 2019); however, there was no experimental component examining the $\mathrm{B}_{12}$-responsive metabolism of $P$. antarctica in laboratory cultures. This study addresses that gap.

We conducted laboratory-based experiments with Phaeocystis antarctica strain CCMP 1871 to examine changes in its transcriptome and proteome in response to vitamin $\mathrm{B}_{12}$ availability under low and high iron conditions. The combination of iron and cobalamin stress was chosen over other nutrients because of observed colimitation in Antarctic seawater communities, specifically of Fe- $\mathrm{B}_{12}$ limitation in diatoms (Bertrand et al., 2007, 2012). A multifactor experimental design enabled the examination of morphotypes' $\mathrm{B}_{12}$-responses in cultures that were predominantly flagellates (low iron) or colonial (high iron). We confirm that this strain is not auxotrophic for vitamin $\mathrm{B}_{12}$, as determined by growth rate and regulation of key $\mathrm{B}_{12}{ }^{-}$ related proteins involved in methionine and folate cycles (a key part of C1 metabolism). We also present the set of significantly differentially abundant proteins (DAPs) and discuss their potential metabolic roles. These proteins are candidates for $\mathrm{B}_{12}$-deficiency biomarkers that may depend on life cycle stage. Finally, this study identified a novel putative MetE in $P$. antarctica that is also found across other marine eukaryotes and prokaryotes. The complementary transcriptomic and proteomic approach used in this study is an effective way to examine the physiological response of cultures to environmental stimuli and to assess their genetic potential (Koid et al., 2014). The complex, polymorphic life cycle of Phaeocystis species requires close inspection to understand the ecophysiology of solitary and colonial cells in response to nutrient limitation. A holistic view of $P$. antarctica ecology and its morphotypes is necessary to inform interpretations of field observations and predictive models of community structure.

\subsection{Methods}

\subsubsection{Experimental overview}

P. antarctica strain CCMP 1871 was grown in constant light $\left(200 \mu \mathrm{mol}\right.$ photons $\left.\mathrm{m}^{-2} \mathrm{~s}^{-1}\right)$, at $4{ }^{\circ} \mathrm{C}$, in trace-metal clean culture conditions across a range of six $\mathrm{B}_{12}$ concentrations at low and high Fe, resulting in twelve total co-limitation treatments. The cultures were monitored with subsamples used to measure relative fluorescence units (RFUs); cell counts of single, colonial cells, and numbers of colonies via microscopy; and harvested for proteomes and transcriptomomes.

\subsubsection{Study organism}

All experiments were conducted using axenic cultures of $P$. antarctica strain CCMP 1871 provided by the Allen Lab that was originally acquired from the Provasoli-Guillard 
National Center for Culture of Marine Phytoplankton. The cultures were maintained using trace metal (TM) clean and sterile technique, following (Bender et al., 2018). The samples were treated with antibiotics and were monitored betwteen transfers for bacteria growth using visual inspection (microscopy), DAPI stains, and inoculating subsamples into peptone and yeast broth. Using these methods, no bacteria were observed in the samples. Eliminating potential bacteria, and thus uncontrolled sources of $\mathrm{B}_{12}$ in the cultures, was key to the experiment. The strain CCMP 1871 was chosen because it produces both single cells and colonial morphotypes in culture, as opposed to strain CCMP 1374 (Bender et al., 2018). P. antarctica strain CCMP 1871 was isolated from open ocean waters in the Southern Bellinghausen Sea (Arthur Harbor, Palmer Station, Antarctica $\left.64.7792{ }^{\circ} \mathrm{S}, 64.0575^{\circ} \mathrm{W}\right)$.

\subsubsection{Culturing methods: medium preparation and growth environment}

Semi-continuous batch cultures were grown in biological triplicates $(n=3)$ in $250 \mathrm{~mL}$ polycarbonate bottles, that are opaque to UV light and thus minimize $\mathrm{B}_{12}$ photodegradation under the grow lights. The methods follow both (Bender et al., 2018; Saito and Goepfert, 2008). The empty culture bottles first underwent a trace metal (TM) cleaning procedure and microwave sterilization. The base culture was made of filtered, microwave-sterilized oligotrophic seawater amended with a $F / 2$ recipe for macronutrients and vitamins (minus cyanocobalamin) and trace metal stock (minus $\mathrm{FeCl}_{3}$ ), using a modified metal buffer concentration of $10 \mu \mathrm{M}$ EDTA, following Sunda and Huntsman (Bender et al., 2018; Sunda and Huntsman, 1995). Macronutrients, vitamins (biotin and thaimine, and vitamin $\mathrm{B}_{12}$ used in media preparation were treated with Chelex-100 resin to remove trace metal contaminants and were filter-sterilized $(0.2 \mu \mathrm{m})$ prior to addition into culture media. The concentration of $\mathrm{Fe}$ ' (and other metals) was set by the $10 \mu \mathrm{M}$ EDTA $\left(\mathrm{Fe}^{\prime} / \mathrm{Fe}_{\text {total }}=0.039\right)$. The cultures were grown in $4{ }^{\circ} \mathrm{C}$ under continuous light at $200 \mu \mathrm{mol}$ photons $\mathrm{m}^{-2} \mathrm{~s}^{-1}$ to emulate summer growth conditions in Antarctica surface waters. For reference, mean mixed-layer irradiances in the Ross Sea range from $(96 \pm 58)$ to $(180 \pm 110) \mu \mathrm{E} \mathrm{m}^{-2} \mathrm{~s}^{-1}$ in the mid spring to summer period (Sedwick et al., 2000).

Cultures of $P$. antarctica strain CCMP 1871 were grown under twelve treatments, a low and high iron condition and a six order-of-magnitude range of $\mathrm{B}_{12}$ concentrations. The initial cultures were acclimated to two iron and six $\mathrm{B}_{12}$ concentrations for at least five transfers (until growth rates stabilized) prior to growth rate and proteome experiments. Two concentrations of dissolved inorganic iron was 120 and 1200 pM Fe', set by the metal buffer EDTA (Sunda and Huntsman, 1995). These iron concentrations were chosen based on prior work that demonstrated a biphasic response in P. antarctica strain CCMP 1871 that contained majority single, flagellated cells at low iron treatments $(2,31,120 \mathrm{pM} \mathrm{Fe}$ ') and majority colonial cells at high iron treatments $\left(740,1200,3900 \mathrm{pM} \mathrm{Fe}\right.$ ') (Bender et al., 2018). $\mathrm{B}_{12}$ treatments were chosen to explore a wide range of environmental concentrations that included $0,10^{-14}$, $10^{-13}, 10^{-12}, 10^{-11}, 10^{-10} \mathrm{M}$ spanning a range of field observations of sub $\mathrm{pM}$ to $\mathrm{pM}$ 
pelagic standing stocks of $\mathrm{B}_{12}$ to laboratory culture standard concentration of $100 \mathrm{pM}$ cyanocobalamin. In this report, the different iron treatments are referred to as low Fe (120 pM Fe') and high Fe (1200 pM Fe').

Growing cultures were monitored via subsamples in $5 \mathrm{ml}$ borosilicate tubes $(13 \times 100 \mathrm{~mm})$ to measure relative fluorescence units (RFUs) and cell counts. Once in mid-to-late exponential phase, the cultures were harvested for proteome, metal quota, and transcriptome analysis. Throughout the harvest process, cultures were maintained on ice and sampled using TM clean and sterile pipetting methods described above. To harvest the cellular biomass, all treatments were centrifuged into pellets that were immediately stored at $-80^{\circ} \mathrm{C}$. Samples were thawed prior to protein extraction. A subsample of the culture was pelleted for metal quotas. A final subsample was filtered on $0.2 \mu \mathrm{m}$ nucleopore filter (treated with RNA later), flash frozen with liquid nitrogen, and stored at $-80^{\circ} \mathrm{C}$ prior to transcriptomic analysis. A Palmer-Maloney counting chamber was used to count cells with a Zeiss Axio Plan microscope on 400× magnification to determine the number of single cells, cells in colonies, and number of colonies. Counts are the average from 10 fields of view.

\subsubsection{Quantifying growth rates}

Growth was quantified using two approaches: fluorometry and microscopy cell counts. Using sterile and TM clean technique to minimize contamination, subsamples were taken throughout the growth period using sterile pipettes, TM clean rinses, while under a culture hood in a class-100 clean room (Saito Lab, WHOI). The subsamples of $P$. antarctica triplicate cultures were collected by keeping culture bottles on ice and using sterile pipettes with an acid rinse $(3 \times 10 \% \mathrm{HCl}$ and $3 \times \mathrm{pH} 2)$ to not contaminate the culture vessels. Subsamples were limited in order to preserve culture biomass for future analysis. These subsamples were aliquoted into $5 \mathrm{~mL} 13 \times 100 \mathrm{~mm}$ borosiicate tubes to measured relative fluorescence units (RFUs) using a TD400 fluorometer and subsequently used for microscopy-based cell and colony counts. Specific growth rate (1/day) was calculated over the linear phase of semi-log plots of RFU or cell density over time, following the equation:

$$
\mu=\ln \left(N_{t} / N_{0}\right) / t
$$

It should be noted that the measurements of $P$. antarctica growth rates, either by in vivo relative fluorescence or cell counts, is confounded by its colony formation that affects fluorescence and complicates the cell counts due to its spherical shape (Saito and Goepfert, 2008). Consequently, microscpy-based counts of cell density were used to report the growth rates.

\subsubsection{Microscopy cell counts}

The same subsamples of $P$. antarctica triplicate cultures used for fluorometry were kept on ice to remain cool and $100 \mu \mathrm{L}$ were pipetted onto a Palmer-Maloney counting chamber with a grid. Once placed onto the slide, the samples were allowed to warm 
and settle to the bottom of the counting chamber to facilitate counts using a Zeiss Azio Plan microscope at $400 \times$ magnification $(40 \times$ lens and $10 \times$ eyepiece). A minimum of 10 random fields of view were used to count cells. Colonies were quantified by identification of a colony outline around collections of cells without flagella with a minimum of $n=2$ cells to identify a colony. A microscopy-based cell counting method was chosen to preserve intact colonies of $P$. antarctica, as opposed to flow cytometric methods that either require dissolution or physical disruption of the colony structure in order to count cells. Counts of single, flagellated cells and counts of colonial cells were used to calculate morphotype growth rates within a single treatment. Cell size during mid-to-late exponential phase (close to time of harvesting cell pellet) was determined using the Zeiss 4.8.2 software and a calibrated scale bar.

\subsubsection{Proteomics: Extraction, MS, Analysis (Global and Targeted)}

Proteins from the harvest cell pellets (one per replicates (3) for each of the 12 Fe- $\mathrm{B}_{12}$ treatments, totalling 36 proteomes for this experiment) were extracted following the magnetic bead protein extraction, alkylation, and reduction protocol and analyzed by liquid chromatography-mass spectrometry (LC/MS/MS) Thermo Fusion mass spectrometer and a Michrom Advance HPLC with a Thermo Flex source. The protein extraction, quantification, reduction, alkylation, purification, and digestion along with peptide recovery, preparation, and injection into the mass spectrometer were conducted using a modified protocol based on (Hughes et al., 2014) that is reported in step-by-step detail in the methods section of Saito et al. (2020). A shotgun proteomics approach was used to analyze the global proteome of $P$. antarctica under the iron and $\mathrm{B}_{12}$ treatments. Tryptic peptides were analyzed via liquid chromatography tandem mass spectrometry (LC/MS/MS), following methods in (Bender et al., 2018). A later set of MS2 scans were performed on the selected peptides to measure low-abundance $\mathrm{B}_{12}$-related proteins. Often the abundance of $\mathrm{B}_{12}$-related proteins is low in culture samples. The selected reaction monitoring method was used to target and quantify the peptides of interest (MetE, MetH, and others). An initial target list was created based on 1D sample searches, and a tandem mass spectrometer (Fusion) was set to inject MS2 scans within $25 \mathrm{ppm}$ of the target list masses. The targeted peptide (for MetE protein) fragmentation spectra, example chromatograms of MS2 intensities as well as with +1 and +2 mass addition for isotopic distributions are shown in the chapter text.

The raw mass spectra were searched against the $P$. antarctica CCMP 1871 experiment translated transcriptome assembly using Proteome Discoverer 2.1 SEQUEST HT algorithm (Thermo, Inc.). The processed files were loaded into Scaffold (version 4.8.4, Proteome Software). The protein identification criteria included a protein threshold False Discovery Rate (protein FDR) of 1.0\%, 1 minimum peptide, and tolerance of SEQUEST 10ppm for the peptide probability threshold. The MS/MS proteomics data were processes using Scaffold (version 4.8.4, Proteome Software). In this study, the protein spectral counts are reported in two ways: (1) normalized 
spectral count for a given protein in each treatment, and (2) spectral counts divided by protein length to yield the spectra abundance factor (SAF) that is normalized by total counts per sample, yielding the NSAF values. Normalization of protein spectral abundances were done using normalized spectral abundance factor (NSAF) and calculated in Scaffold (Zybailov et al., 2006). The NSAF normalization method accounts for the MS bias that longer proteins often have more peptide identifications than shorter proteins. Protein and peptide reports were exported for later analysis and visualization in $\mathrm{R}$ and Python scripts.

The R-package PLGEM was used to determine significantly differentially abundant proteins (DAPs) between $\mathrm{B}_{12}$ treatments; and done so in step-by-step mode with the NSAF spectral counts of proteins (Pavelka et al., 2004, 2008). The power law global error model (PLGEM) statistical method describes the variance-versus-mean dependence in normalized proteomic data. The PLGEM model was fit on the low iron, $-\mathrm{B}_{12}$ condition and the analysis yielded a a slope of 0.799 , adjusted $r^{2}$ of 0.997, and Pearson correlation of 0.953. Next, DAPs were derived using a statistical hypothesis testing framework, testing against the null hypothesis that proteins are not differentially expressed between treatments. For the low iron and high iron treatments, the respective $-\mathrm{B}_{12}$ condition was used as the baseline for comparison (low iron $\mathrm{B}_{12}$, high iron $\mathrm{B}_{12}$ ). In the comparisons between treatments, proteins were considered significantly differentially abundant (DAP) if the $P$-value, calculated from signal-tonoise ratios, was below 0.05 ; resulting in an estimated false positive rate (FPR) of $5 \%$. The PLGEM method has been applied successfully to microarray gene expression data and laboratory proteomic experiments of marine microbes (Cohen et al., 2018; Walworth et al., 2016).

\subsubsection{RNA extraction, Illumina sequencing, and annotation}

Total RNA was isolated from $P$. antarctica cell cultures filtered onto $0.2 \mu \mathrm{m}$ filters and immediately flash frozen with liquid nitrogen and stored at $-80^{\circ} \mathrm{C}$ (one filter per treatment, three biological replicates of two iron concentrations and six cobalamin concentrations, for a total of 36 transcriptomes). The samples were processed by the Allen Lab. Sequencing was performed using (Illumina HiSeq platform). A transcriptomic assembly was generated for the culture samples, following methods described in (Bender et al., 2018). As there is no available reference genome for $P$. antarctica strain CCMP 1871, the RNA-seq reads were assembled with a de novo approach. The downstream reads were trimmed for quality and filtered. Contigs and predicted open reading frames (ORFs) were assembled. The ORFs were annotated de novo via KEGG, KO, KOG, PFam, and TIGRFam assignments. Additionally, taxonomic classifications were assigned to each ORF. The reason for the de novo assembly is that strain CCMP 1871 does not yet have a complete genome. As a result, many different expressed partial fragments may be assembled into the same gene, especially those without homologous protein gene hits. Given the protein abundance patterns, the genome was reanalyzed to search for a putative MetE gene domain. 


\section{RNA-Seq analysis}

Transcriptomics via RNA-Seq was used to study the behavior of $P$. antarctica genes under varying iron and $\mathrm{B}_{12}$ conditions. The transcriptomic reads were aligned back to the reference transcriptome. The raw reads counts were normalized using the median of ratios method to interpret gene expression patterns between conditions and to identify significantly differentially expressed genes (DEGs). Briefly, the median of ratios method calculates the ratio of the expression level between samples for each gene and then the median ratio for all genes is used as an internal normalization. The median of ratios method is appropriate for comparing gene counts between samples for differential expression analysis. This normalization and a PCA analysis was conducted using the R package DESeq2 (Anders and Huber, 2010).

\subsubsection{Identification of a novel putative MetE}

The transcriptomic assembly contained two contigs that were annotated as MetE and were differentially expressed under $-\mathrm{B}_{12}$ and $+\mathrm{B}_{12}$ conditions in the transcriptome and proteome. The transcript models were revisited and an in silico construct based on alignment with the putative MetE contigs was identified in the transcriptomic assembly. The full in silico reconstructed from transcripts and the resulting protein was annotated using InterPro. Several transcript models were combined based on alignments that created an open reading frame with multiple protein domains. One in silico construct was made per transcriptomic library.

\subsubsection{Construction of phylogenetic tree of putative MetE}

A phylogenetic analysis was used to inspect the presence of the putative MetE in haptophytes and other microbes. The in silico MetE sequence was searched in the NCBI non-redundant protein (NCBI-nr) database, the Marine Microbial Eukaryote Transcriptome Sequencing Project (MMETSP) database, and the assemblies of two additional haptophytes Chrysochromulina tobinii and Emiliania huxleyi from JGI using the blastP algorithm with an e-value cutoff of $1 \mathrm{e}-5$. The tree was manually pruned to remove distantly related sequences and minimize long branch attraction, with the exception of canonical diatom MetE sequences to demonstrate their dissimilarity with this putative MetE.

$P$. antarctica sequences were recovered from four independent transcriptomic datasets, including two unpublished CCMP1871 datasets, one CCMP1374 unpublished dataset and one dataset from MMETSP (MMETSP1100). CCMP1871 sequences were in silico reconstructed based on high-similarity nucleotide alignment with fully contiguous sequences from CCMP1374 and MMETSP1100. Amino acid sequences for phylogenetic analysis were retrieved from NCBI-nr and PhyloDB using DIAMOND BLAST (Buchfink et al., 2015). Outgroup (non-haptophyte) sequences were clustered on $50 \%$ identity and $80 \%$ coverage using MMseqs2 (Steinegger and Söding, 2017). Datasets were aligned by MAFFT v7.407 (Katoh and Standley, 2013) using the

L-INS-i refinement and a maximum of 1000 iterations and trimmed by trimAl v1.4 
(Capella-Gutiérrez et al., 2009) allowing $70 \%$ sequences have a gap (-gt 0.3). Maximum likelihood (ML) trees were inferred by IQ-TREE v1.6.12 (Nguyen et al., 2015) using the $\mathrm{LG}+\mathrm{C} 20+\mathrm{F}+\mathrm{G}$ model and the posterior mean site frequency method (Wang et al., 2018), starting from a $\mathrm{LG}+\mathrm{F}+\mathrm{G}$ guide tree and employing the strategy of rapid bootstrapping followed by a "thorough" ML search with 1,000 ultra-fast bootstrap replicates.

\section{$2.3 \quad$ Results}

This study used quantitative transcriptomic and proteomic methods to investigate the metabolic response of Phaeocystis antarctica morphotypes to cobalamin availability. A multifactor experimental design was used to evaluate the $\mathrm{B}_{12}$-dependent changes in $P$. antarctica morphotypes, by using a low and high iron concentration (hereafter -Fe and $+\mathrm{Fe}$ ) that result in a predominantly flagellate and colonial ratio in culture, as previously demonstrated (Becquevort et al., 2007; Bender et al., 2018). These cultures were tested across a wide range of $\mathrm{B}_{12}\left(0,10^{-14}, 10^{-13}, 10^{-12}, 10^{-11}, 10^{-10} \mathrm{M}\right)$ to assess their metabolic response to a range of plausible environmental $\mathrm{B}_{12}$ concentrations (Sañudo-Wilhelmy et al., 2006, 2012). All results presented are purified P. antarctica biological triplicate cultures grown for each $\mathrm{Fe}-\mathrm{B}_{12}$ condition using trace-metal methods and sterile technique to maintain axenic conditions. A total of 36 samples resulted from the two iron, six $\mathrm{B}_{12}$, and three replicates. Cells were harvested at mid- and lateexponential growth phase, as depicted in the growth curves (Supplemental Fig.2-13). The analysis is focused on low iron and high iron treatments in absence and abundance of $\mathrm{B}_{12}$ (hereafter $-\mathrm{B}_{12}$ and $+\mathrm{B}_{12}$ ), from the no addition and replete $\mathrm{B}_{12}$ conditions. For comparison, $-\mathrm{B}_{12}$ reflects starvation conditions and $+\mathrm{B}_{12}$ is replete conditions of $100 \mathrm{pM}$ was used, the latter is a standard amount used in typical laboratory algal cultures that is roughly $100 \times$ higher than natural seawater concentrations (Panzeca et al., 2006). The following sections examine the morphotype ratio, growth rates, and global and targeted proteomic and transcriptomic exploration of gene products found to be significantly different between the $-/+\mathrm{Fe},-/+\mathrm{B}_{12}$ conditions.

\subsubsection{Morphotype ratio is controlled by iron and not $B_{12}$}

Microscopic examination of the triplicate cultures was conducted for cell cycle analysis, measuring total cell density at the time of harvest, and the growth rate of cultures. The ratio of $P$. antarctica morphotypes is controlled by iron availability and not $\mathrm{B}_{12}$. Low iron treatments were predominantly in the single cell morphotype compared to colonial cells dominating the cultures in high iron conditions (Fig.2-1). These findings corroborate results from Becquevort et al. (2007) and Bender et al. (2018), implying the influence of $\mathrm{Fe}$ on colony formation is reproducible. It is important to note that both morphotypes coexisted in all treatments except for low iron $10^{-12}$ and $10^{-11} \mathrm{pM}$ $\mathrm{B}_{12}$ conditions, in which only the single-cell, flagellated morphotype was observed $(100 \%)$. In contrast to iron, $\mathrm{B}_{12}$ does not appear to exert an influence over life cycle stage in $P$. antarctica. This experimental design, however, does enable the exploration 

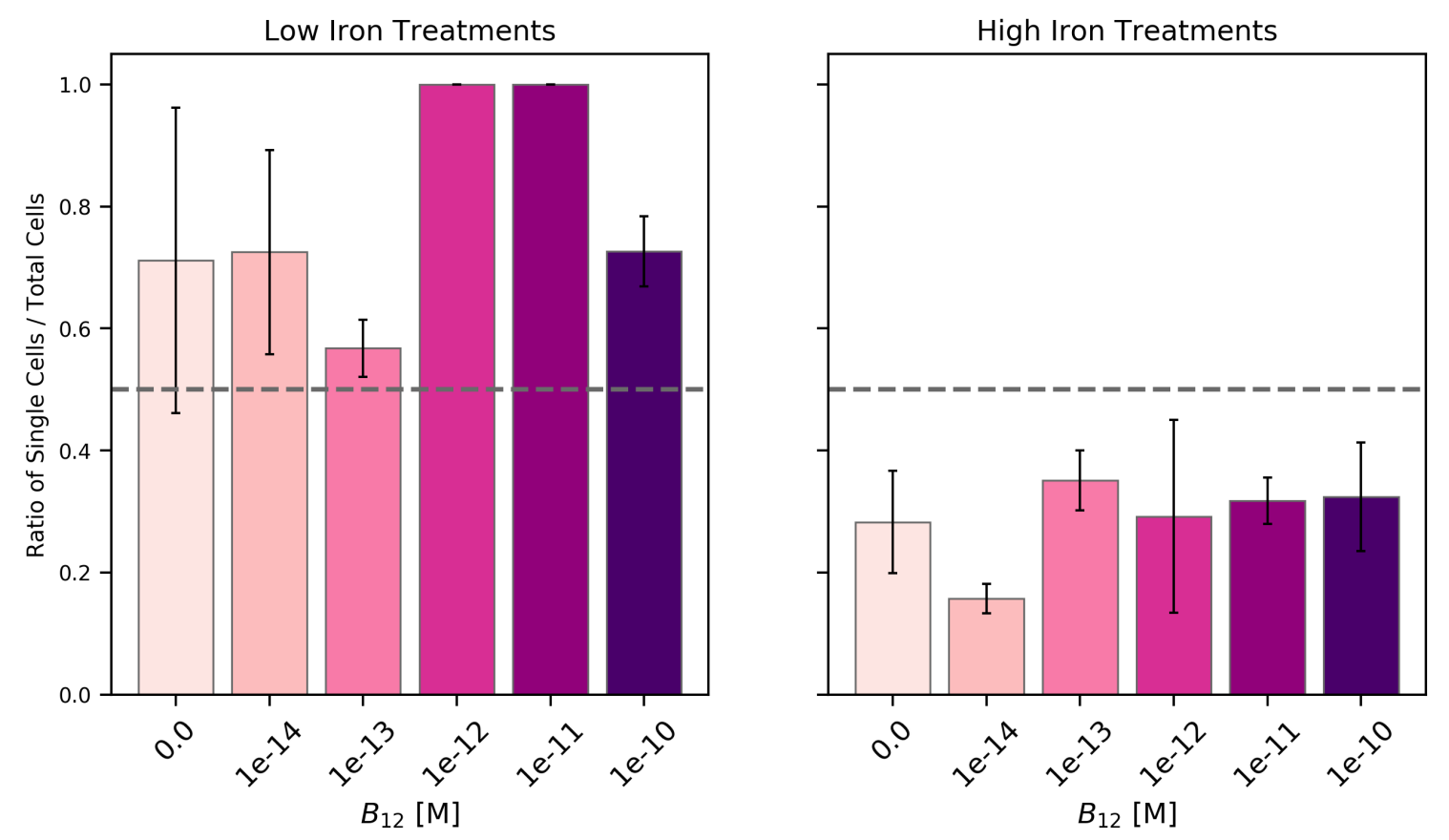

Figure 2-1: Ratios of $P$. antarctica single cells to total cells at the time of harvest, as the morphotype ratios under low and high iron and across the full range of $\mathrm{B}_{12}$ concentrations. The dashed horizontal line demarcates the $50 \%$ threshold of single cells to total cells counted. The error bars are the standard deviation of the single cell ratio of three biological replicate culture samples. The low iron cultures were predominantly in the single cell morphotype, including two treatments being solely solitary cells $\left(10^{-11}\right.$ and $\left.10^{-12} \mathrm{~B}_{12}\right)$, while the high iron cultures were predominantly colonial.

of morphotype-specific responses to varying $\mathrm{B}_{12}$ concentrations given the dominance of different life cycle stages under specific iron conditions.

\subsubsection{Morphotypes' growth rates vary in response to $\mathrm{B}_{12}$}

$P$. antarctica growth rates in response to $\mathrm{B}_{12}$ varied differently between iron conditions (Fig.2-2). Under low iron, the highest growth rates were in the middle range of $\mathrm{B}_{12}$ concentrations, corresponding to observed environmental $\mathrm{B}_{12}$ concentrations ( 0.1 to 1 pM) (Panzeca et al., 2009). Under high iron, growth rates were stable across most treatments, but have a marked increased under replete conditions at $100 \mathrm{pM}$. Growth rates ranged from $<0.2 \mathrm{~d}^{-1}$ to approaching $0.4 \mathrm{~d}^{-1}$, within the range observed in other $P$. antarctica growth studies under stimilar light conditions (Bender et al., 2018). The

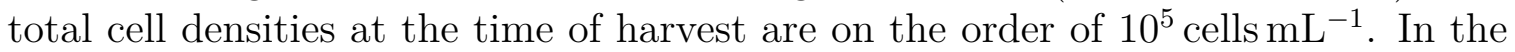
low iron experiment, higher $\mathrm{B}_{12}$ treatments had higher cell densities than high iron conditions, corresponding to a higher ratio of solitary cells. The -Fe- $\mathrm{B}_{12}$ cultures had the lowest cell densities, while the $+\mathrm{Fe}+\mathrm{B}_{12}$ culture had a higher cell density. Growth rate was measured two ways, via cell counts and relative fluorescence units (RFU). Given the changes in cell size, morphotype, and potential confounding effects of RFU 

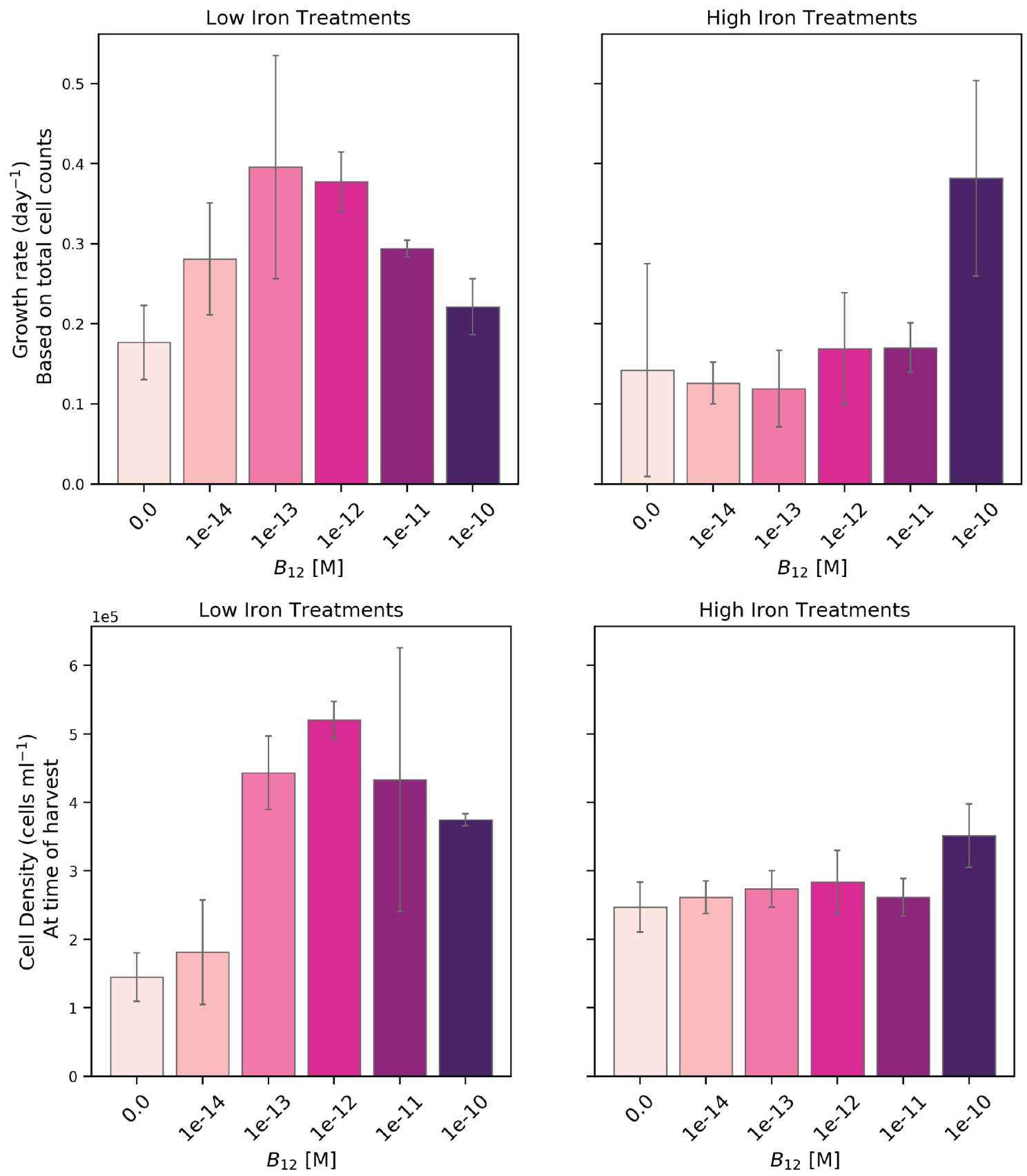

Figure 2-2: Growth rates (top panel) and cell density at the time of harvest (bottom panel). Under low iron, the highest growth rates were in the middle ranges of $\mathrm{B}_{12}$ treatements, corresponding to seawater concentrations. Under high iron, growth rate was stable across $\mathrm{B}_{12}$ treatments, but there is a marked increased in the replete condition at $100 \mathrm{pM} \mathrm{B} \mathrm{B}_{12}$.

measurements (self-shading, different cell sizes, and chlorophyll per cell), the growth rates used for the analysis are from microscopy cell counts. 


\subsubsection{Morphotype ratio accounts for most of the transcrip- tomic variability between treatments}

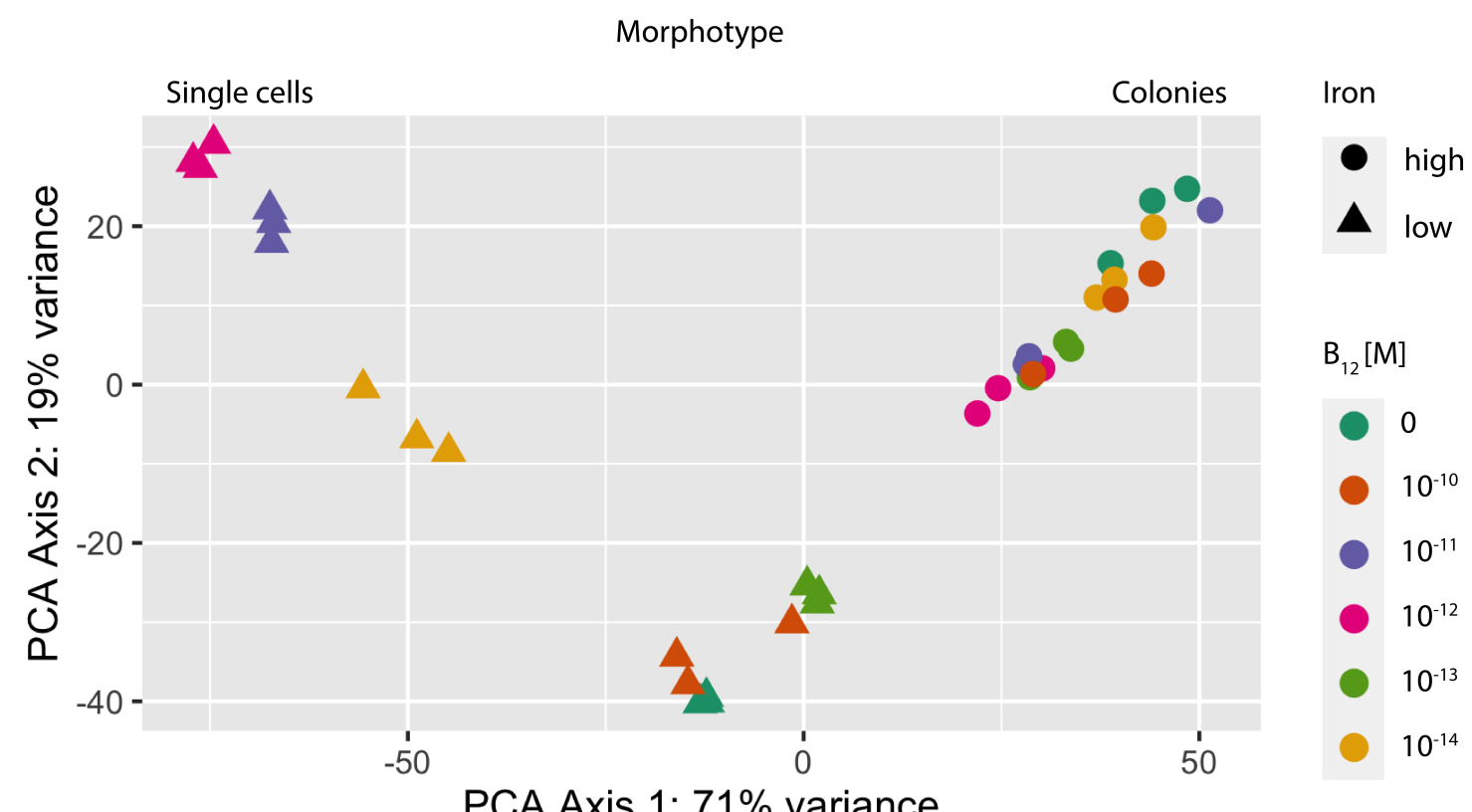

Figure 2-3: A principal component analysis (PCA) of RNA-seq data for the multi-factor Fe- $\mathrm{B}_{12}$ experiment on $P$. antarctica. The two principal component axes explain $71 \%$ and $19 \%$ of the variability in the transcriptome of $P$. antarctica under these conditions. Biological replicates cluster closely, while colonial and solitary replicates cluster separately. The majority of variance is between samples rather than within biological replicates. Principal axis 1 corresponds to the morphotype ratio, with the purely single cell cultures on the top left and colonial cultures on the top right. Principal axis 2 corresponds to $\mathrm{B}_{12}$ conditions, with the low-iron solitary cells having a larger variance in their transcriptome in response to vitamin concentrations compared to the high-iron treatments. The PCA is performed on distances between samples derived from regularized log transformed counts.

A principal component analysis (PCA) of the normalized RNA-seq data revealed two principal component axes that explain $71 \%$ and $19 \%$ of the variability in the transciptome of $P$. antarctica under the experimental range of iron and $\mathrm{B}_{12}$ conditions (Fig.2-3). The PCA reveals that the RNA expression patterns between biological replicates are consistent, as indicated by the close spatial clustering of Fe$\mathrm{B}_{12}$ treatments, thereby lending confidence the experimental results. In other words, the majority of variation is between samples and not within biological replicates. Broadly, PCA axis 1 corresponds to the gradient between morphotypes: from the purely single-cell cultures (top left) to colonial cultures (top right). The high iron treatments cluster together, indicating that these predominantly colonial cultures are more similar to one another than to the flagellate cells in low iron treatments. The low iron treatments show a greater variance long the PCA axis 2, which corresponds to $\mathrm{B}_{12}$ treatment. The low-iron experiment cultures had more transcriptome variance in response to $\mathrm{B}_{12}$, whereas iron-iron cultures varied minimally. That colonial and 
solitary cells' transcriptomes cluster separately was also observed in P. globosa cultures (Mars Brisbin and Mitarai, 2019).

\subsubsection{P. antarctica regulates methionine synthase transcripts and proteins in response to $B_{12}$}

Gene expression and protein abundance analysis determined that $P$. antarctica modulates both methionine synthase isoforms in response to $\mathrm{B}_{12}$ availability. Under high cobalamin availability, the $\mathrm{B}_{12}$-dependent methionine synthase (MetH) isoform was more abundant; under low cobalamin availability, two putative $\mathrm{B}_{12}$-independent methionine synthase (MetE) contigs were more abundant (Fig.2-4). This implies that $P$. antarctica expresses MetE to replace MetH when $\mathrm{B}_{12}$ is scare, as seen in both the transcript expression and protein abundance. Overall MetE gene expression and protein abundances are about 10 times higher than the levels of MetH, consistent with our knowledge of the biochemistry of these two methionine synthase isoforms; specifically that MetH has a much high catalytic activity, thereby requiring fewer enzymes to produce the same amount of methionine (Gonzalez et al., 1992). Consequently, a $\mathrm{B}_{12}$-limited metabolism would need many more copies of MetE to achieve the same methionine production (Banerjee and Matthews, 1990). In order to cope with cobalamin stress, $P$. antarctica likely increased MetE to satisfy methionine synthase demand. When $\mathrm{B}_{12}$ is scare, a trade-off between MetH to MetE would reduce overall cobalamin demand and support methionine synthase production. This inter-replacement has also been observed in the $\mathrm{B}_{12}$-flexible diatom P. tricornutum and green alga C. reinhardtii (Bertrand et al., 2012; Bunbury et al., 2020).

The $\mathrm{B}_{12}$ concentration at which metH is up-regulated and MetH is more abundant varies with iron and morphotype. For the predominantly solitary cells in low iron, the trade-off between MetE and MetH proteins happens at lower $\mathrm{B}_{12}$ concentration, starting at $1 \mathrm{pM} \mathrm{B} \mathrm{B}_{12}$ versus $100 \mathrm{pM}$ in the high-iron colonial cultures. This difference may reflect a quota of $\mathrm{B}_{12}$ that morphotypes require to switch to using the MetH enzyme over MetE. From the ecophysiological perspective, it stands to reason that the smaller, motile solitary morphotype may be able to respond to lower environmental concentrations of $\mathrm{B}_{12}$, while the larger colonial morphotype may require a larger $\mathrm{B}_{12}$ concentration to transition to using MetH.

RNA-seq analysis revealed that the patterns of metE and metH transcript expression broadly match the corresponding protein abundances, with the exception of high MetH transcript expression under the $0 \mathrm{M} \mathrm{B}_{12}$ concentration in low iron, with no corresponding protein. This protein-transcript mismatch is curious, but not surprising given the potential decoupling between protein and transcript abundance in eukaryotic algae (Wurch et al., 2011). Taken together, these analyses reveal that the MetH and MetE proteins and transcripts have coordinated expression across most $\mathrm{B}_{12}$ concentrations and between the two morphotypes in low and high iron conditions. $P$. antarctica maintains MetE proteins and transcripts, even with sufficient $\mathrm{B}_{12}$ and the expression of MetH, suggesting a utility to having both methionine synthase isoforms. Maintaining MetE is advantageous given the scant and variable $\mathrm{B}_{12}$ concentrations 

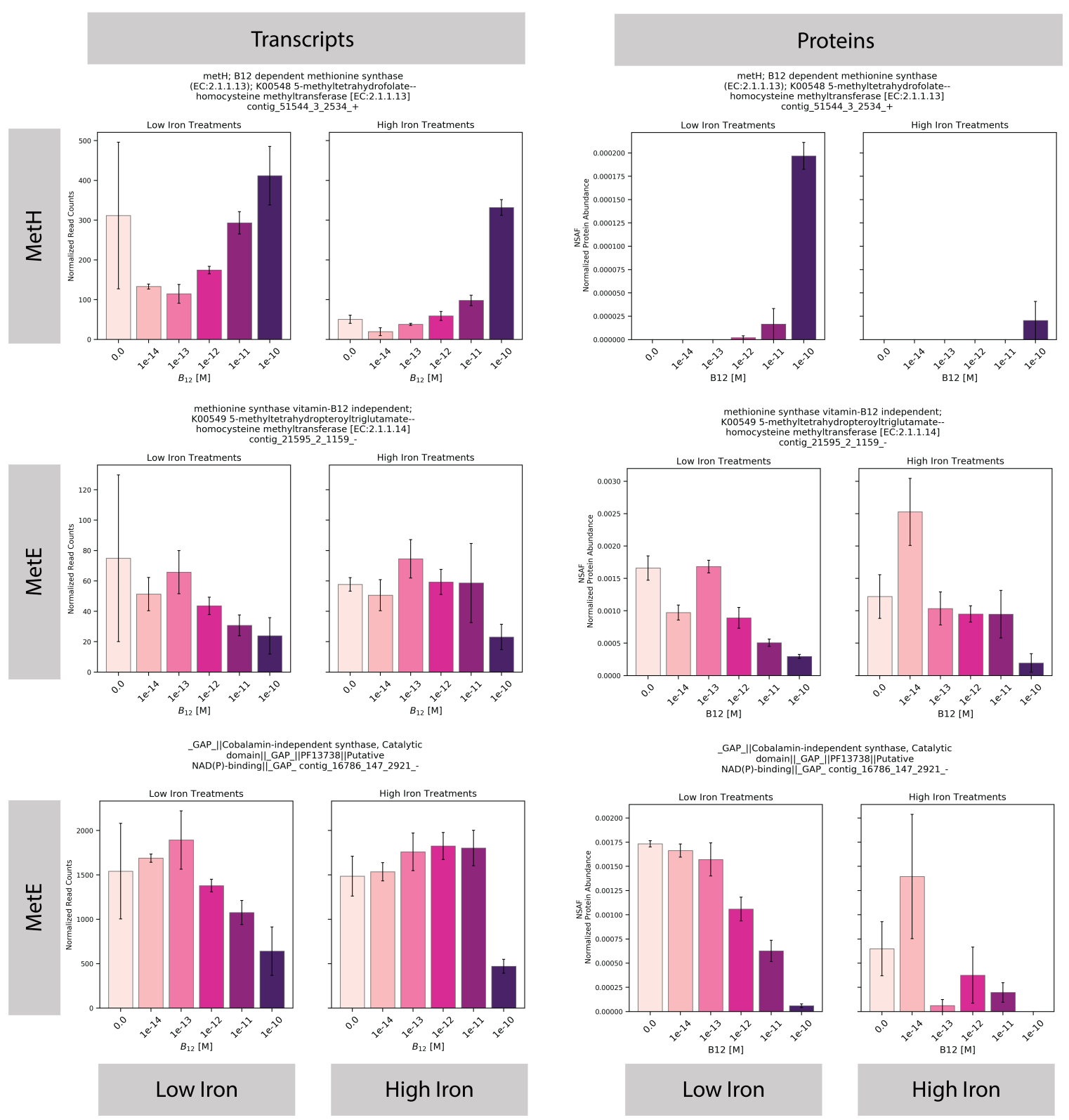

Figure 2-4: Patterns of the normalized transcript expression and normalized peptide abundance of both isoforms of methionine synthase: two contigs for putative $\mathrm{B}_{12}$-independent methionine synthase (MetE) and one contig for $\mathrm{B}_{12}$-dependent methionine synthase (MetH). MetH is much more abundant under high $\mathrm{B}_{12}$ availability, while MetE is much more abundant under low $\mathrm{B}_{12}$ availability. The bars are the mean values of three biological triplicates and the error bars represent the standard error. The two MetE protein sequences are contig_21595_2_1159_- and contig_16786_147_2921_-, and the MetH protein sequence is contig_51544_3_2534_+ from the transcriptomic assembly for P. antarctica CCMP 1871 . The contig identifiers and protein sequences are included in the Supplemental Materials (Section 2.6) to this chapter. The gene and protein annotations from the transcriptomic assembly are included for completeness. 
in natural systems and may be essential for other biological processes related to the methionine cycle (like the synthesis of DMSP, protein, and DNA and methylation reactions) or perhaps for different processes in localized in organelles, like the flagellar MetE in C. reinhardtii (Pazour et al., 2005; Schneider et al., 2008).

\subsection{5 $\mathrm{B}_{12}$-regulated proteomic shifts involve $\mathrm{C} 1$ metabolism and cell cycle regulation}

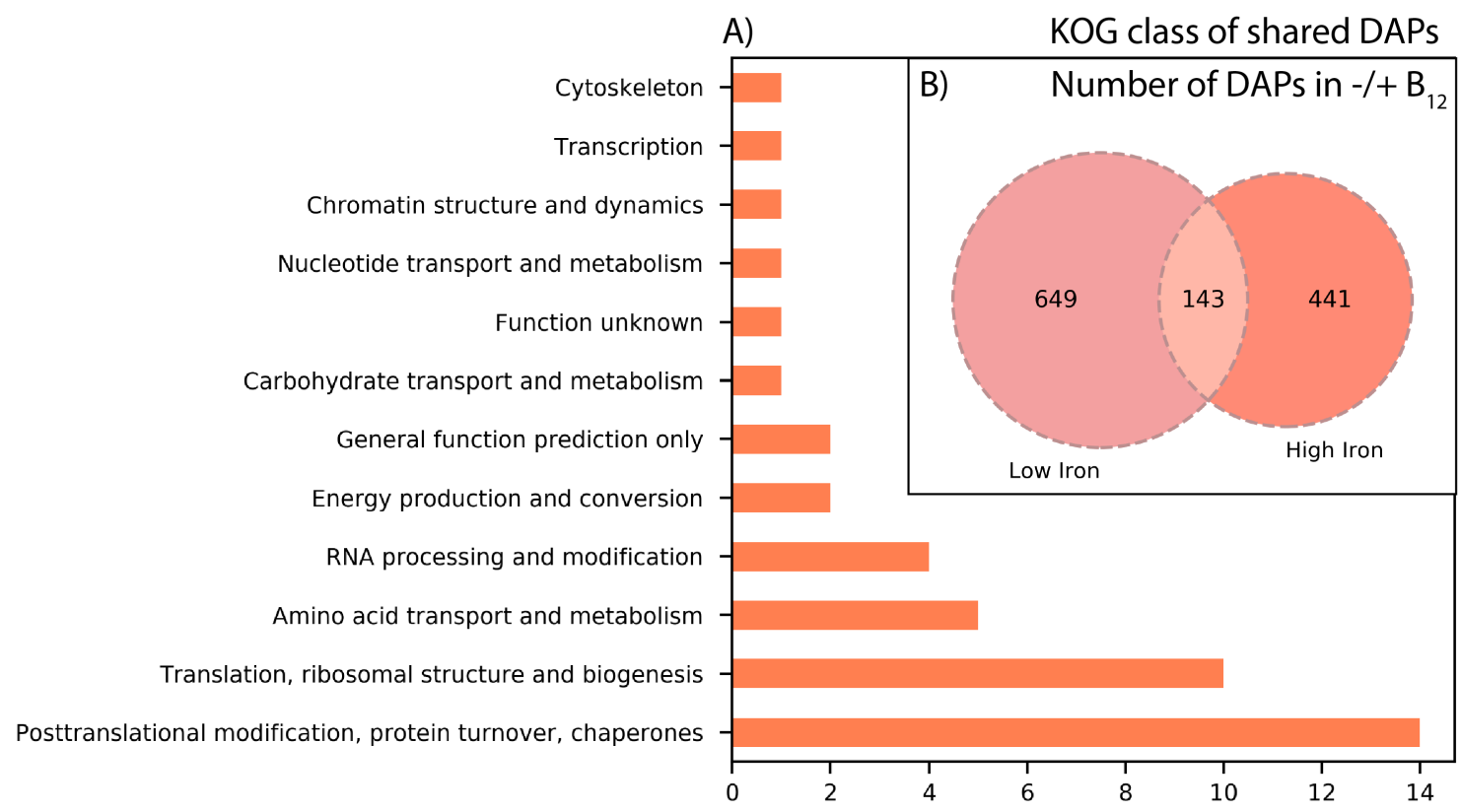

Figure 2-5: (A) A count of the KOG classifications of the shared differentially abundant proteins (DAPs) between low and high iron treatments for $-/+\mathrm{B}_{12}$ comparisons. (B) a Venn Diagram of the unique and shared DAPs between $-/+\mathrm{B}_{12}$ under high and low iron conditions. The DAPs were determined using PLGEM with $P<0.05$.

In this section, the broad proteome changes in response to $-/+\mathrm{B}_{12}$ are examined under the low and high iron conditions, respectively. In order to identify significantly differentially abundant proteins (DAPs) between treatments, the power law global error model (PLGEM) was used with a significance threshold of $P<0.05$. This approach is especially useful to identify low-abundance but significantly variable proteins. The $\mathrm{B}_{12}$-responsiveness under high and low iron treatments reflects morphotype-specific changes that may influence the ecophysiology and community dynamics of $P$. antarctica in nature. This is explored in scatter plots of normalized protein abundances under $-/+\mathrm{B}_{12}$ treatments for low and high iron and also via MA plots to compare the average vs. fold change protein abundances between comparisons. The scatter plots serve to highlight the proteins uniquely expressed under $-/+\mathrm{B}_{12}$ (along the axes), with zero values in one treatment, which are otherwise excluded from the MA-plot as a consequence of fold-change calculations. Finally, the observed DAPs in P. antarctica 
are juxtaposed with a study that used an affinity-based $\mathrm{B}_{12}$-probe to identify proteins to which $\mathrm{B}_{12}$ was directly bound in the bacterium Halomonas (Romine et al., 2017). That study revealed a suite of hitherto unexamined $\mathrm{B}_{12}$-related proteins (e.g., enzymes involved in folate, ubiquinone, and methionine metabolism and also methyltranferases and chemotaxis)(Romine et al., 2017). To our surprise, several DAPs in P. antarctica directly matched to those tagged with the $\mathrm{B}_{12}$-probe, and we expand the list of possibly $\mathrm{B}_{12}$-associated and even $\mathrm{B}_{12}$-regulated proteins with enzymes corresponding to those functional pathways. These proteins are are strong candidates for further study in marine eukaryotes.

$P$. antarctica cultures shared 143 DAPs expressed in $-/+\mathrm{B}_{12}$ conditions, across both low and high iron (Fig.2-5) (PLGEM, $P<0.05$ ). Low iron cultures had more DAPs (649) in response to $-/+\mathrm{B}_{12}$ than high iron cultures (441), in agreement with more variable low-iron treatments found in the PCA analysis (Fig.2-3). Included in the 143 shared DAPs, were several $B_{12}$-related proteins and methyltransferases. For the DAPs that had annotations, KOG class functional categories are summarized in Fig.2-5. The largest categorical changes under $-/+\mathrm{B}_{12}$ treatments are for RNA and protein metabolism, which is expected given the role of $\mathrm{B}_{12}$-related proteins in methylation reactions and protein synthesis. Many proteins were annotated as "hypothetical proteins" or lacked annotations altogether. These proteins are good candidates for future work to examine the possibility of $\mathrm{B}_{12}$-transporter proteins and other $\mathrm{B}_{12}$-related functions. Of the annotated proteins, three broad categories emerged as being responsive to $\mathrm{B}_{12}$ : methionine cycling and methyltransferases, cell cycle related proteins, and iron-related proteins. In this discussion, available $\mathrm{KO}$ numbers are included from the annotated transcriptomic assembly.

\section{$\mathrm{B}_{12}$-starvation causes major shifts in $\mathrm{C} 1$ metabolism}

Overall, the Carbon 1 (C1) metabolic pathway was responsive to $\mathrm{B}_{12}$, as summarized in Fig.2-6. Readers are encouraged to examine Fig.2-6 for this section. Methionine synthase plays a pivotal role in $\mathrm{C} 1$ metabolism and connects the methionine and folate pathways, thereby affecting both protein and nucleotide synthesis and overall cellular methylation capacity. Both MetH and MetE catalyze the parallel synthesis of methionine (Met) and tetrahydrofolate (THF) from homocystein (Hcy) and 5'-methyltetrahydrofolate (5- $\mathrm{CH}_{3}$ - THF). S-adenosylmethionine synthetase (MetK/AMS1) converts methionine to $S$-adenosylmethionine (SAM), a universal cofactor involved in methylation reactions and even the remethylation and reductive repair of cobalamin used in MetH (Drennan et al., 1994). SAM enables the transfer of methyl groups to DNA, proteins, lipids, and secondary metabolites via SAM-dependent methyltransferase proteins. A consequence of methionine cycling is that methylated proteins like histones are indirectly affected by $\mathrm{B}_{12}$-availability. As a result of methyl transfers SAM is converted to $S$-adenosylhomocysteine (SAH). Finally, $S$-adenosyl homocysteine hydrolase (SAH1) hydrolyzes SAH to homocysteine, which can then go through MetE/MetH to become methionine.

In our experiment, all enzymes in the methionine cycle were DAPs, as were many folate cycle proteins. As presented earlier, MetH and putative MetE are responsive to 


\section{A)}

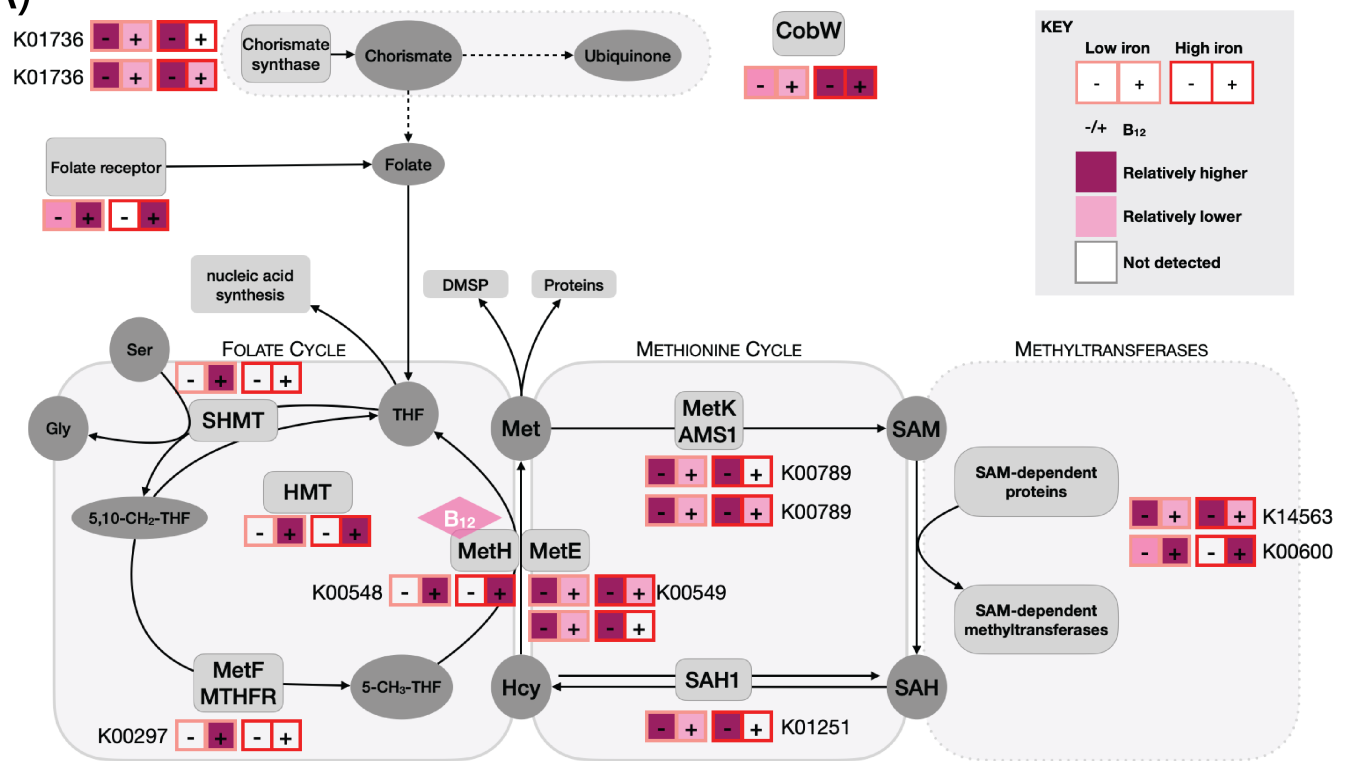

B)

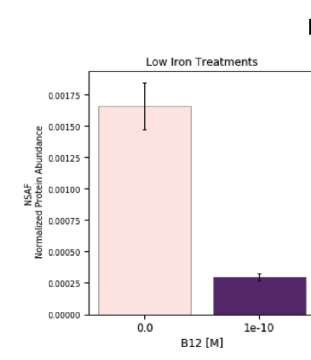

MetE
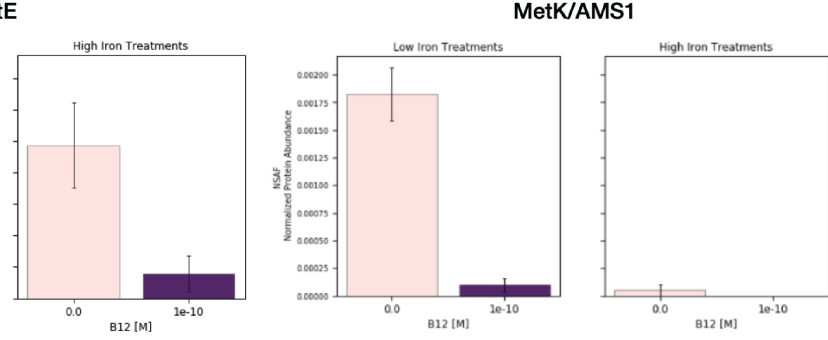

SAH1
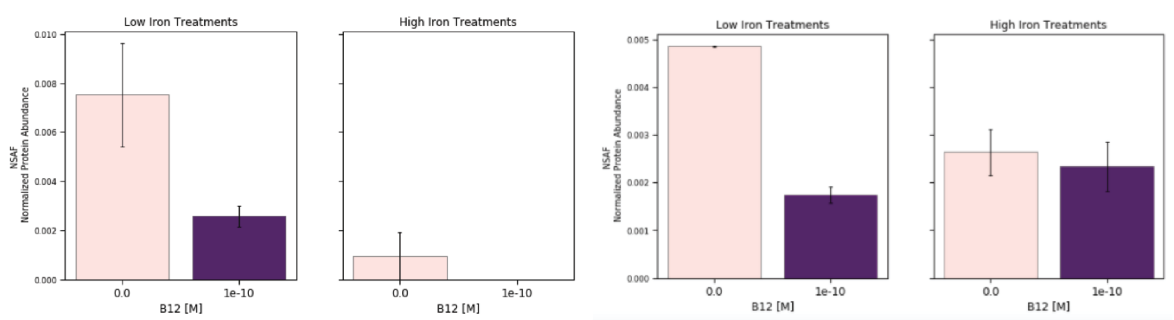

Figure 2-6: The $\mathrm{C} 1$ metabolism had several differential abundant proteins (DAPs) between $-/+\mathrm{B}_{12}$ treatments. (A) the metabolic map of $\mathrm{C} 1$ metabolism, focused on MetE and MetH. Metabolites are in dark grey ovals. Enzymes are in light grey rectangles. Solid arrows indicate enzyme-catalyzed reactions. Dashed arrows indicate pathways. The key is provided on the top right to interpret the protein abundance changes among C1-metabolism DAPs. (B) The normalized protein abundances for some of the methionine cycle DAPs: MetE, MetK/AMS1, and SAH1. The error bars are the standard error of NSAF protein values. Key metabolite and enzyme abbreviations are described in the chapter text. KEGG identifiers are included for proteins that had corresponding annotations.

$\mathrm{B}_{12}$ availability, in an expected way. In - $\mathrm{B}_{12}$ conditions, all methionine-cycle proteins are more abundant. This is likely in an attempt to maintain methionine and SAM production and homocysteine levels, despite reduced efficiency of the MetE methionine 
synthase isoform. Specifically, MetE, MetK/AMS1, and SAH1 are up-regulated under $-\mathrm{B}_{12}$. These proteins were similarly down-regulated under $+\mathrm{B}_{12}$ conditions, likely due to the increased efficiency of MetH (Fig.2-6). If SAM becomes limiting it can hamper overall cellular methylation, so there is a need for cells to maintain produce SAM via MetK regardless of $\mathrm{B}_{12}$ availability; additionally SAH1 activity is needed to regulate $\mathrm{SAH}$ and homocysteine levels. This coordinated changes in the methionine cycle agree with previous studies of $\mathrm{B}_{12}$-flexible marine eukaryotic algae, namely $P$. tricornutum and C. reinhardtii that have both MetE and MetH (Bertrand et al., 2012; Bunbury et al., 2020; Helliwell et al., 2014). Some of these studies also observed methyl folate trapping as a consequence of $\mathrm{B}_{12}$-limitation and methionine stress; specifically up-regulation of increased serine hydroxymethyltransferase (SHMT) in P. tricornutum under - $\mathrm{B}_{12}$ (Bertrand et al., 2012). Although P. antarctica did not express proteomic signatures of folate trapping, several folate-related proteins were differentially abundant: homocysteine S-methyltransferase (HMT), a folate receptor, and chorismate synthases, notable because chorismate is the precursor to folate. Finally, $P$. antarctica had several methyltransferase DAPs that are likely SAM-dependent, including: homocysteine S-methyltransferase [K00547], glycine hydroxymethyltransferase [K00600], arsenite methyltransferase, O-methyltransferase, and a histone-lysine N-methyltransferase [K09187]. These broad $\mathrm{C} 1$ metabolic responses to $\mathrm{B}_{12}$ are further evidence that the putative MetE in $P$. antarctica indeed has the function of a $\mathrm{B}_{12}$-independent methionine synthase.

Interestingly $\mathrm{B}_{12}$ affected the regulation of several iron-related proteins included several TonB-dependent receptors and TonB-related proteins (MotB/ExbB) and an Fe-S related ABC transporter ATPase subunit SufC [K09013]. TonB proteins have been previously observed in $P$. antarctica cultures, though the TonB system is typically associated with prokaryotes (Bender et al., 2018). It is possible that these proteins were acquired via horizontal gene transfer. A study of $E$. coli-which has both MetE and MetH-found mutual inhibition of cobalamin and siderophore uptake systems, suggested competition for TonB-dependent transport proteins as the mechanism (Greene, 1996; Kadner and Heller, 1995). They report that TonBdependent transporters could become saturated in the presence of high levels of iron and cobalamin, limiting the uptake of both. To overcome this limitation, TonBreceptors and TonB-transporters would be expected to increase when $\mathrm{B}_{12}$ and/or Fe are high. In $P$. antarctica, there are signatures of iron-limitation under $+\mathrm{B}_{12}$ conditions, including the up-regulation of TonB system proteins and iron stress biomarkers (ISIP2 and ISIP3). Future studies should examine the nature of these TonB proteins in $P$. antarctica along with their effect of $\mathrm{B}_{12}$ on iron transport, given the potential importance for micronutrient limitation in Antarctic waters.

Finally, a range of cell-cycle related proteins were also significantly differentially abundant. For example, flagellar proteins (FAP162 and FAP173), cell division protease (FtsH [K03798]), and elongation factors G [K02355], Ts [K02357], and Tu [K02358]. SAM has a speculated role in cell division through its utility as a methyl donor. Although examined in prokaryotes, a study involving E. coli MetK/AMS1-knockout mutants observed that when MetK activity was low, cells produced long filaments and had methylated Tu proteins, which deliver aminoacyl-tRNA to the ribosome 
during translation (Newman et al., 1998). Elongation factors (Tu, Ts, and G) and cell division proteases $(\mathrm{FtsH})$ were DAPs with some of the highest fold change values between $-/+\mathrm{B}_{12}$, as seen in Fig.2-9, although with opposite patterns. In low iron, the predominantly flagellate cells appear to have more cell-division proteins under high $\mathrm{B}_{12}$; while high iron colonial cells expressed these proteins more under low $\mathrm{B}_{12}$. These results suggest that $B_{12}$ impacts cell-cycle regulation differently in flagellate versus colonial morphotypes, but this trend warrants more analysis.

\section{Aside: Comparison of $\mathrm{B}_{12}$-responsive differentially abundant proteins to a study of $\mathrm{B}_{12}$-bound proteins highlights novel pathways influenced by the vitamin}

Beyond the expected C1 cycling proteins, this study identifies several other $\mathrm{B}_{12^{-}}$ responsive proteins in $P$. antarctica with lesser known biochemical functions, highlighted in Figs.2-7, 2-8, and 2-9. These DAPs had a strong correspondence to a set of 41 proteins identified with a $\mathrm{B}_{12}$-based chemical probe in a study by Romine et al., 2017 that have regulatory roles in folate metabolism, ubiquinone biosynthesis, methionine metabolism, and chemotaxis (Romine et al., 2017). Although that study focused on a heterotrophic bacterium Halomonas sp. HL-48, the captured $\mathrm{B}_{12}$-bound proteins are found across biological domains and are an important comparison point for this study. The unexpected $\mathrm{B}_{12}$-bound proteins in Halomonas are involved in DNA, RNA, and protein synthesis, revealing a larger metabolic role for $\mathrm{B}_{12}$ in overall cell metabolism. In the following discussion, I highlight a set of DAPs in P. antarctica that correspond to proteins discovered by the $\mathrm{B}_{12}$-probe in Halomonas (Romine et al., 2017).

To begin the comparison, the primary DAPs of interest are proteins in the related cycles of methionine and folate, and also ubiquinone. Methionine synthase proteins MetH and MetE were also found via the $\mathrm{B}_{12}$-probe, though curiously only MetH is confirmed to use $\mathrm{B}_{12}$ as a cofactor. MetE translation is known to be regulated in many bacteria by a cobalamin-binding riboswitch, but these authors also suggest another control mechanism via allosteric interaction (binding of an effector molecule to an enzyme at a site other than the active site) of MetE and $\mathrm{B}_{12}$ (Kazanov et al., 2007; Romine et al., 2017). Proteins involved in the folate cycle were also differentially abundant. Though only one common protein was identified via the $\mathrm{B}_{12}$-probe: methylenetetrahydrofolate reductase (MetF [K00297]). In P. antarctica, other DAPs include a folate receptor family protein and two chorismate synthase contigs (both corresponding to [K01736]), which are of interest because chorismate is a precursor to both folate and ubiquinone.

$P$. antarctica DAPs did not overlap with $\mathrm{B}_{12}$-probe ubiquinone biosynthesis proteins, but the overall pathway is still worth comparing since the $\mathrm{B}_{12}$-responsiveness of ubiquionine processes was not known prior to that study. Ubiquitin is a regulatory protein involved in protein interactions, degradation, localization, and can even alter their enzymatic activity. Ubiquitin and ubiquitin-conjugating enzyme E2 N [K10580] was a shared DAP. Low iron cultures had additional ubiquitin-related DAPs, including ubiquitin-activating enzyme protein (ThiF), ubiquitin [K04523], NADH:ubiquinone 


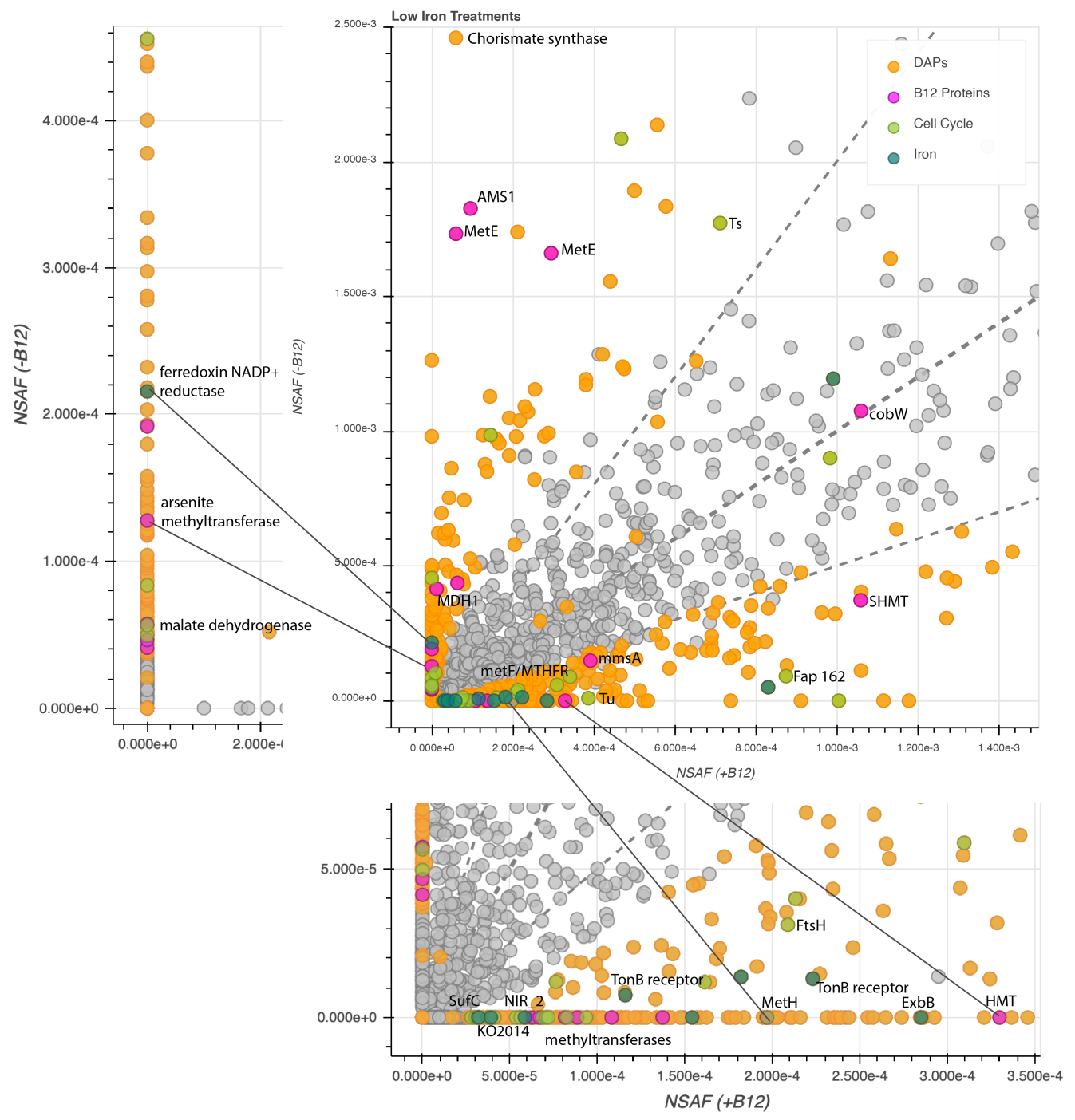

Figure 2-7: Shotgun MS analysis of $P$. antarctica under low iron in $-/+\mathrm{B}_{12}$ treatments $\left(0 \mathrm{M}\right.$ and $\left.10^{-10} \mathrm{M}\right)$ reveals $\mathrm{B}_{12^{-}}$responsive proteins. Each point is an identified protein mean value (biological replicates $n=3$ ) as the normalized spectral abundance (NSAF) values of one treatment plotted against another treatment. The dashed lines demarcate the 2:1, 1:1, and 1:2 abundance ratios. Colored dots are proteins that were found to be significantly differentially abundant (PLGEM, $P<0.05$ ), with $\mathrm{B}_{12}$-related proteins highlighted in pink, cell-cycle related proteins in green, and iron-related proteins in teal, all other DAPs are in yellow. The central scatter plot and side panels are zoomed in to aid in the detection of low abundance DAPs. Select proteins are annotated to facilitate discussion, for example, the methionine synthase isoforms (MetE and MetH). The reader is encouraged to examine the proteins along the axes that are present/absent depending on $\mathrm{B}_{12}$ availability.

oxioreductase [K11352], ubiquitin carboxyl-terminal hydrolase [K11836], and ubiquinol cytochrome c reductase [K00416]. To my knowledge, these findings of this Fe- $\mathrm{B}_{12}$ study 


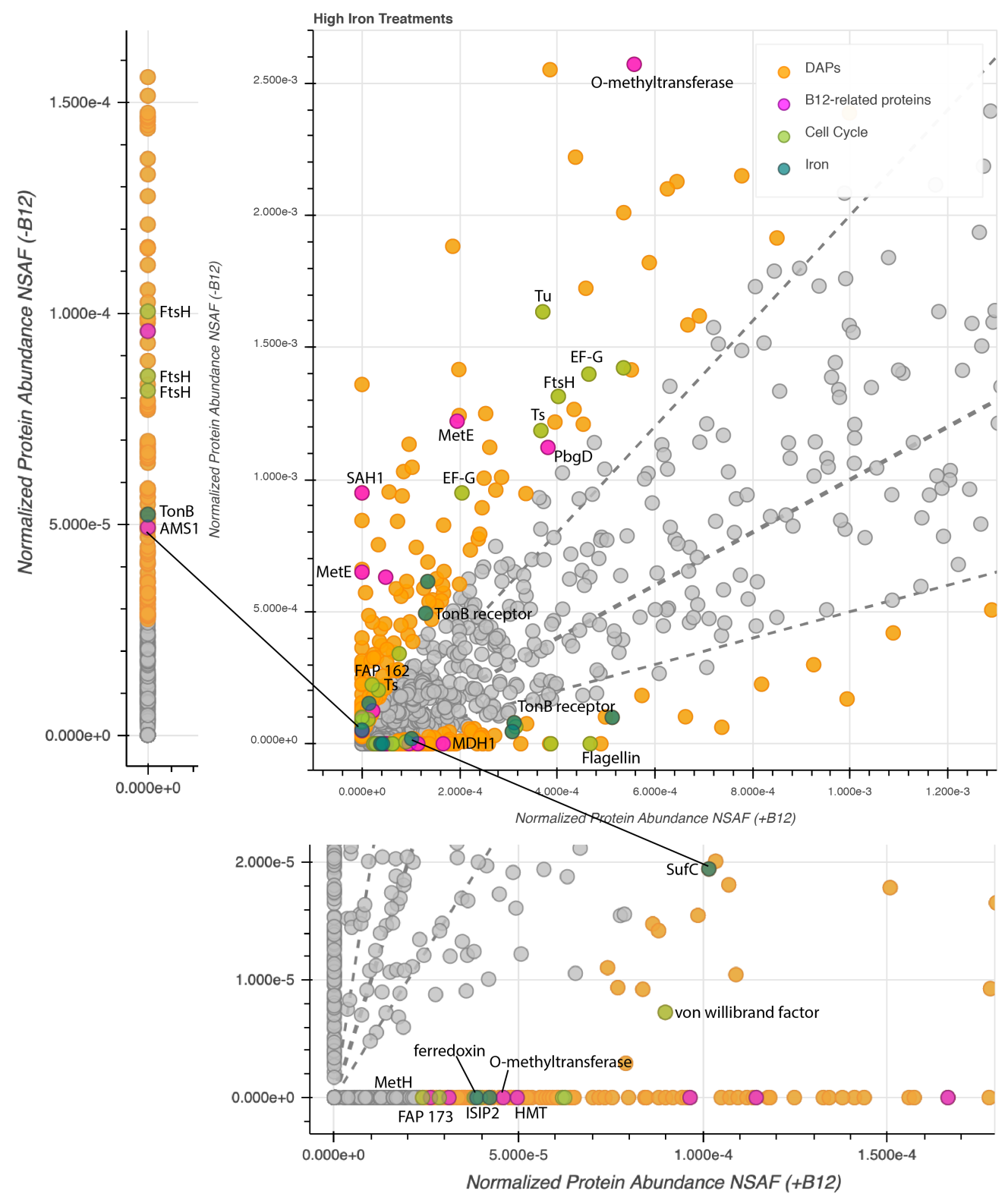

Figure 2-8: Shotgun MS analysis of $P$. antarctica under high iron in $-/+\mathrm{B}_{12}$ treatments

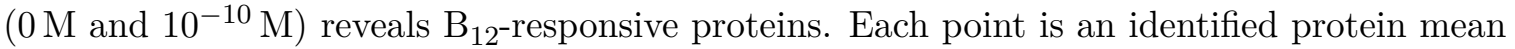
value (biological replicates $n=3$ ) as the normalized spectral abundance (NSAF) values of one treatment plotted against another treatment. The dashed lines demarcate the 2:1, 1:1, and 1:2 abundance ratios. Colored dots are proteins that were found to be significantly differentially abundant (PLGEM, $P<0.05$ ), with $\mathrm{B}_{12}$-related proteins highlighted in pink, cell-cycle related proteins in green, and iron-related proteins in teal, all other DAPs are in yellow. The central scatter plot and side panels are zoomed in to aid in the detection of low abundance DAPs. Select proteins are annotated to facilitate discussion, for example, the methionine synthase isoforms (MetE and MetH). The reader is encouraged to examine the proteins along the axes that are present/absent depending on $\mathrm{B}_{12}$ availability. 


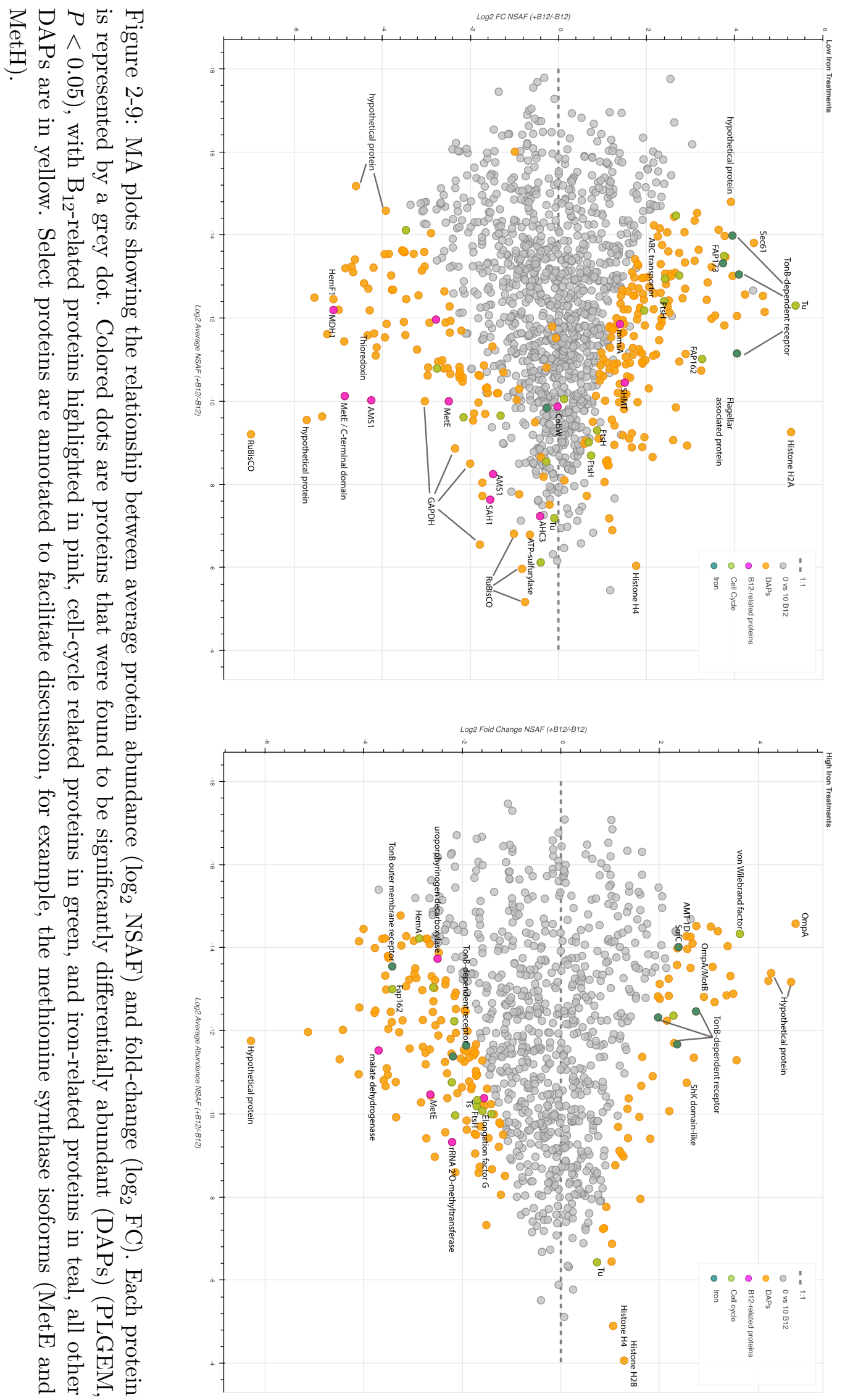


in $P$. antarctica are only the second reported instance of $\mathrm{B}_{12}$ affecting ubiquinone metabolism other than (Romine et al., 2017).

Additionally, nearly the entire Heme biosynthesis pathway was observed to be differentially abundant in response to $\mathrm{B}_{12}$ and those proteins are summarized in Supplemental Fig.2-14. Heme biosynthesis is a fundamental metabolic pathway that leads to the biosynthesis of chlorophylls, phycobilins, cytochromes, and vitamin $\mathrm{B}_{12}$. The $\mathrm{B}_{12}$-probe labeled uroporphyrinogen decarboxylase (HemE), which catalyzes the first reaction in heme biosynthesis from uroporphyrinogen III - notably the metabolite precursor to $\mathrm{B}_{12}$ and chlorophyll biosynthesis. These results hint that $\mathrm{B}_{12}$ has a regulatory role, perhaps as an allosteric controller, in directing biosynthesis of heme and its byproducts like chlorophyll, phycobilins, and cytochromes, as suggested by (Romine et al., 2017).

Finally, some DAPs of unknown function found in $P$. antarctica were and also identified via the $\mathrm{B}_{12}$-probe. Some of the highest fold-change proteins in $+\mathrm{B}_{12}$ under high iron were were OmpA/MotB family proteins, which are of unconfirmed function in $P$. antarctica, but are required for cell-to-cell interactions in E. coli (Movva et al., 1980). Similarly CobW is responsive to $\mathrm{B}_{12}$, but while classified as a $\mathrm{B}_{12}$-biosynthesis protein its function is only hypothesized to be involved in coordination of the cobalt atom in the corrin ring structure of $\mathrm{B}_{12}$. These proteins are highlighted in Figs.2-7, 2-8 and 2-9. The $\mathrm{B}_{12}$-probe labeled a chemotaxis sensor protein that was not detected in this study. However, two flagellar proteins were differentially abundant (FAP 162 and FAP 173), along with the previously described set of cell-cycle related proteins. As a final note, an oxidoreductase protein methylmalonate-semialdehyde dehydrogenase (MmsA) was more abundant in $+\mathrm{B}_{12}$ in P. antarctica and was also found via the $\mathrm{B}_{12^{-}}$ probe method. Many of the proteins reported in the $B_{12}$-probe study were previously not known to be influenced by $\mathrm{B}_{12}$ availability. The DAPs identified in $P$. antarctica as responsive to $\mathrm{B}_{12}$ include many of these "surprising" proteins that were bound with $\mathrm{B}_{12}$. In summary, we identified an expected and novel set of $\mathrm{B}_{12}$-influenced proteins in $P$. antarctica that are involved in the methionine cycle (MetE/MetH/MetK/SAH1), heme biosynthesis, methyltransferase reactions, folate cycling (MetF), ubiquinone metabolism, and other systems (OomA/MotB, CobW, MmsA) that await further exploration.

\subsubsection{Identification of novel putative MetE}

Proteomics approaches enabled a thorough examination of the putative MetE contigs in culture samples using targeted mass spectrometry with precursor and fragment ion analysis, as seen in Fig.2-10. In an earlier experimental replicate, a list of select $\mathrm{B}_{12}$-related proteins was first identified along with charge and mass values, which were then used to target these low-abundance proteins in the present experiment with larger sample volumes, and consequently more biomass and protein. This targeted approach resulted in the detection of these low-abundance MetE and MetH proteins and for comparison between sample conditions. Fig.2-10 highlights the putative MetE protein sequences, peptides found via shotgun proteomics (yellow), and two example spectra and chromatograms for unique peptides (highlighted in grey) from 
these proteins. The finding of these proteins prompted the reexamination of the $P$. antarctica transcriptomic assembly to target the putative MetE sequence and examine its neighboring genes.
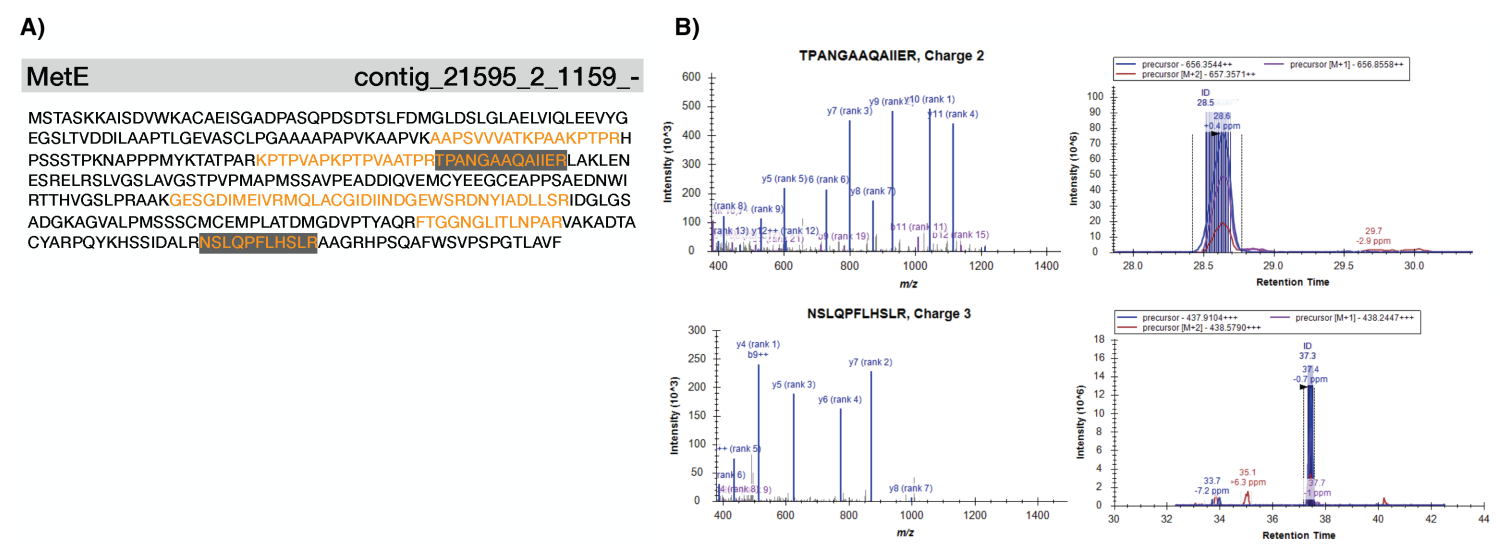

C)

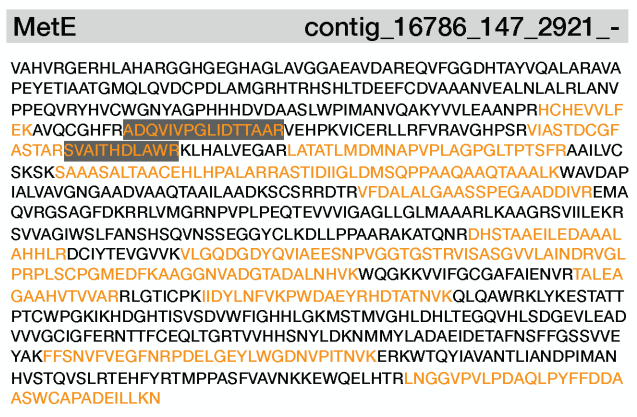

D)

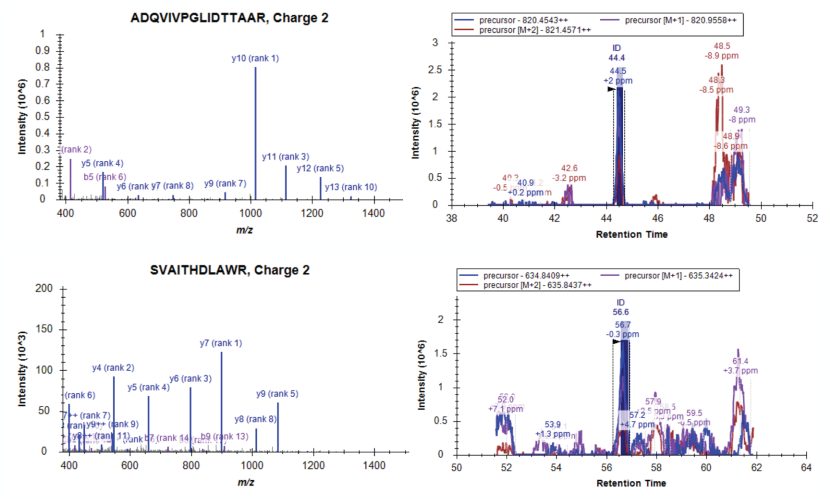

Figure 2-10: Two putative $\mathrm{B}_{12}$-independent methionine synthase (MetE) contigs and their respective amino-acid sequences with highlighted portions in yellow indicating unique, identified tryptic peptides (likely the N-terminal and C-terminal domains) that were observed in the expressed proteome (grey background in panels A and C), example target spectra (left $\mathrm{B}, \mathrm{D}$ ), and chromatograms (right B, D) of fragment ions for two peptides corresponding to putative $P$. antarctica MetE from the experiment cultures. The example chromatograms of MS1 intensities include the +1 and +2 mass addition for isotopic distributions to demonstrate the targets and their utility as potential protein biomarkers of $\mathrm{B}_{12}$-stress in P. antarctica in lab culture and field samples. The two protein sequences are contig_21595_2_1159_- and contig_16786_147_2921_- from the transcriptomic assembly for P. antarctica CCMP 1871.

A novel putative MetE was identified from the transcriptomic assembly and protein abundance data from the culture experiment. Two contigs from the assembly annotated as MetE, were found to be differentially abundant in proteomic samples, and examined further through in silico characterization, leading to the identification of a multi-domain protein construct in the transcriptomic libraries of both $P$. antarctica CCMP 1871 and CCMP 1374. In the Supplemental Material (Section 2.6) to this chapter, the results of a pairwise alignment and percent identity of the two MetE contigs, as well as the percent identity of the MetE contigs and the putative MetE 


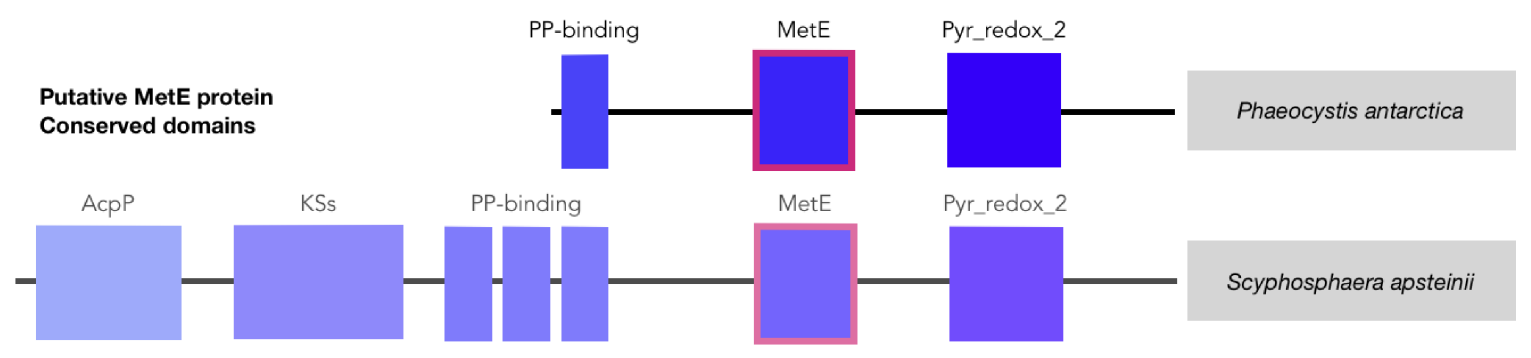

Figure 2-11: Domain arrangement diagram indicating the putative $\mathrm{B}_{12}$-independent methionine synthase (MetE) protein domain in Phaeocystis antarctica species (A) and the closest protein phylogenetic match Scyphosphaera apsteinii (B), for comparison. The query was an in silico construct based on an alignment with the putative MetE contigs identified via proteomics and then examined in the transcriptomic assembly and annotated using InterPro. Conserved domains on the $P$. antarctica in silico sequence include a phosphopantethein attachment site (PP-binding, a prosthetic group that acts as a "swinging arm" for the attachment of amino-acid groups or fatty acids), $\mathrm{B}_{12}$-independent methionine synthase (MetE), and pyridine nucleotide-disulphide oxioreductase (Pyr_redox_2). The query match of $S$. apsteinii also includes further N-terminal conserved domains, acyl transferase (AcpP) and ketoacyl asynthase (KS).

protein are reported. The in silico construct for $P$. antarctica CCMP 1871 was used as the query sequence (Supplemental Fig.2-16) to be searched in NCBI-nr and MMETSP. This constructed sequence, summarized in Figs.2-11 and 2-16 was found intact in P. antarctica strains CCMP 1871, CCMP 1374, and the Caron Lab isolate MMETSP 1100, all with similar sequence length and identity. On this sequence is a UROD/MetE-like domain with a conserved zinc and homocysteine binding residues with a mutated tetrahydrofolate (THF) binding site, another domain further Cterminally has a FAD/NADPH binding function, and the N-terminal domains could bind phosphopantetheine (PP-binding). Most of the protein homologs from eukaryotes (including dinophytes, heterokontophytes, chlorophytes, and labyrinthulids) found in the search are similarly multi-domain proteins consisting of MetE, PP-binding, and FAD/NADPH binding conserved domains. Some matches extend further N-terminally and contain additional domains for ketoacylsynthase and acyltransferase, including the sequence from Scyphosphaera aspteinii- the closest match to the $P$. antarctica sequences. A screenshot of the protein domain arrangement in $S$. aspeinii and $P$. antarctica are included in Supplemental Fig.2-15.

Based on these search results, a phylogenetic tree was constructed for this putative MetE protein (2-12) that demonstrates the following patterns: 1) closely related protein sequences are present in haptophytes - notably P. antarctica, S. apsteinii, E. huxleyi, P. carterae, and C. polylepsis - and also in dinoflagellates, chlorophytes, and hitherto undescribed eukaryotes and 2) bacterial protein homologues are limited to the MetE-like domains, are on a separate deep branch of the phylogenetic tree (with closest bacterial hits to delta-proteobacteria), and include matches to several Antarctic species' proteins (e.g., Granulosicoccus antarcticus and Pseudorhodobacter antarcticus). Notably, the "canonical" MetE of diatoms appears to be a completely different enzyme family that is distantly related from this putative MetE (does not cluster closely); 
but is homologous because it can be aligned on the same tree. That the canonical MetE and this putative MetE have low sequence similarity (and are not a result of convergent evolution) likely explains why prior studies overlooked this putative MetE in haptophytes and concluded that most haptophytes are $\mathrm{B}_{12}$-auxotrophs (Croft et al., 2005; Helliwell et al., 2011; Nef et al., 2019), an important point that will be further considered in the Discussion. The phylogenetic tree including diatoms is presented first to illustrate the homology but distant relation to this putative MetE; the phylogenetic tree without diatoms is in Supplemental Fig.2-17.

These findings of a $\mathrm{B}_{12}$-responsive MetE/MetH trade-off contrast from a recent study that reported that no haptophytes contained MetE (Nef et al., 2019). This previous study was based on a bioinformatic survey of mostly transcriptomic data from the MATOU databse found that all 19 haptophyte species examined only contained MetH and did not contain a canonical MetE or MetE pseudogene (Nef et al., 2019). It should be noted that this in silico analysis searched for the canonical MetE of Chlamydomonas reinhardtii (A8JH37, Uniprot; XP_001702934.1, NCBI) and P. tricornutum (B7G1X4, Uniprot) in transcriptomes that were likely grown under $\mathrm{B}_{12}$-replete conditions, in which cultures may not have expressed MetE. Another study observed that Emiliani huxleyi cultures maintained a consistent growth rate under $-\mathrm{B}_{12}$ and $+\mathrm{B}_{12}$, but these cultures were later confirmed to have associated, antibiotic-resilient bacteria (found both on the calcifying and non-calcifying cells) that were a possible $B_{12}$ source (Helliwell, 2017; Helliwell et al., 2011, 2013). Lastly, another study surveyed 22 haptophytes and concluded that 14 were $\mathrm{B}_{12}$-auxotrophs and 8 did not require $B_{12}$, though only two of these species were grown in the study and the rest were inferred from literature review without confirmation of potential bacterial associates (Croft et al., 2005).

For this putative MetE, the transcript expression, protein abundance, and phylogenetic survey strongly suggest that this protein has the characteristics and expected regulation patterns of functional MetE in response to $-/+\mathrm{B}_{12}$ conditions and is found in eukaryotic taxa that have flagellated life cycle stages, like $P$. antarctica. Our results of a novel putative MetE form are intriguing considering another non-canonical MetE, dubbed core-MetE, was recently identified in bacteria and archaea (Deobald et al., 2020). The core-MetE enzyme appears to be an ancient, minimal isoform that transfers methyl groups from methylcobalamin instead of methyl-tetrahydrofolate (THF) to homocysteine, and therefore does not contain the canonical THF binding domain (Deobald et al., 2020). Future enzyme assays of this putative MetE in $P$. antarctica would confirm the function of this protein.

\section{Phylogenetic tree reveals that the putative MetE is found in flagellated eukaryotes}

There are two key physiological characteristics in common among several protist species on this phylogenetic tree of the putative MetE protein: most have a polymorphic life cycle (multiple life stages) and nearly all have a motile cell stage with flagella. The putative MetE sequences was identified in other eukaryotic marine microbes, including: haptophytes (Phaeocystis, Emiliania, Scyphosphaera, Pleurochrysis, 


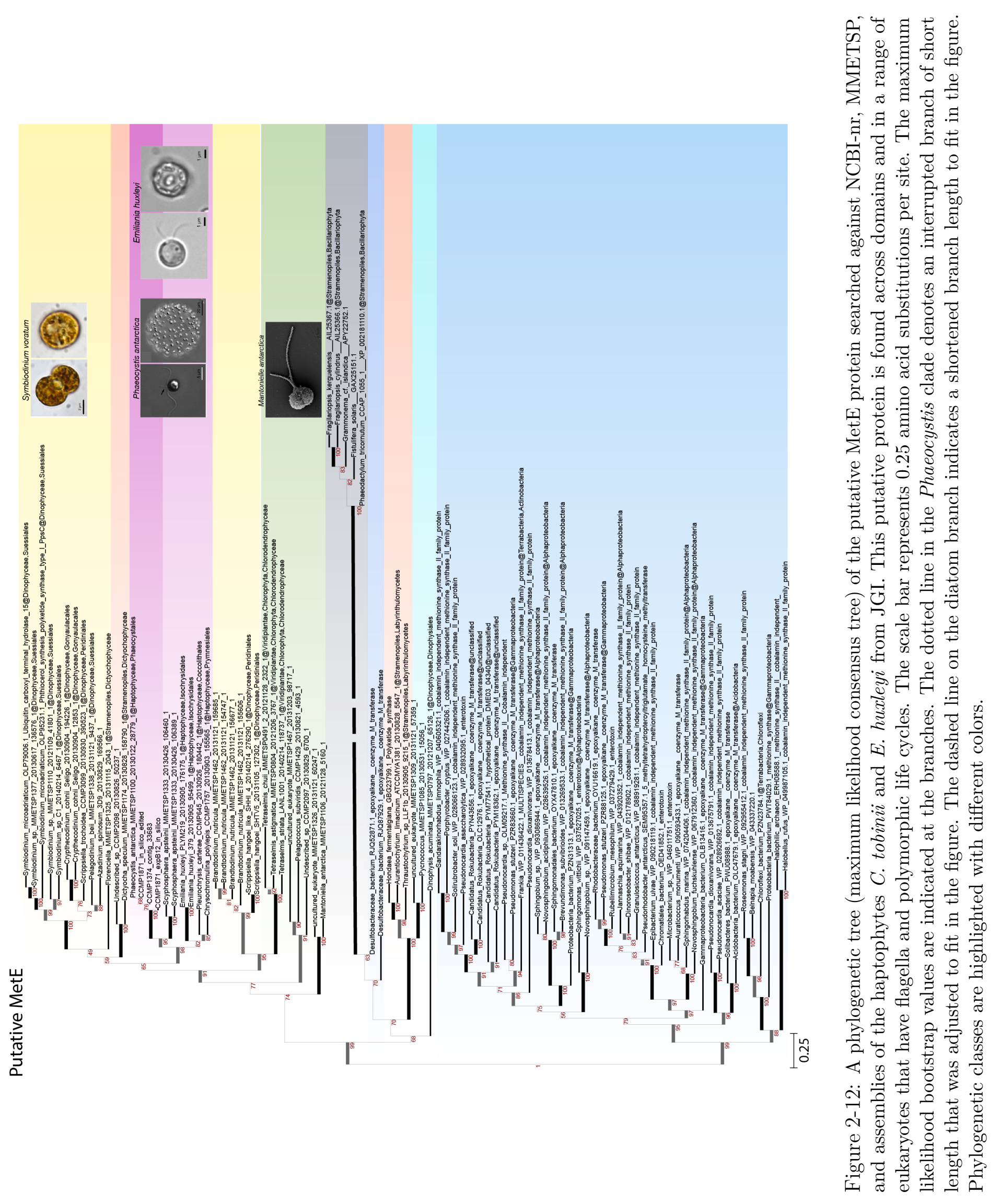


and Chrysochromulina genus), dinoflagellates (Symbiodinium, Crypthecodinium, Scrippsiella, Brandtodinium, Pelagodinium, and Azadinium species), and chlorophytes (Tetraselmis species). More distant matches on a separate branch were found to members of the Labryinthulomycetes of Stramenopiles (a broad group including diatoms and giant kelp), though these proteins they cluster most closely to Dulsulfobacteracaea bacterium epoxyalkane coenzyme M transferase.

There are a few taxonomic groups worth mentioning beyond the close matches to other haptophyte protein sequences. As mentioned, almost all of these protists have a motile, flagellated cell type, and most have a complex, polymorphic lifestyle transitioning between haploid and diploid stages (Phaeocystis and Emiliania) or motile and vegetative cells. Several genuses are known to have colony-forming species (Phaeocystis, Tetraselmis, Pelagococcus, and Aurantiochytrium). And some are known to have symbiotic associations (Symbiodinium microadriacticum, Pelagodinium beii with foraminifera, and Brandtodiniu nuctuiluca with radiolarians). This was a surprising and intriguing pattern amongst the eukaryotic results that warrants closer inspection into the phylogenetic relationships between these groups and possible related metabolic pathways, especially concerning $\mathrm{B}_{12}$, cell cycle regulation, and cell-to-cell communication.

The putative MetE has close matches to several MetE proteins from bacteria and archaea. The tree has a deep branch between eukaryotic and bacteria proteins, with the exception of proteins belonging to Desulfobacteraceae bacteria family, which cluster most closely with the diatom MetEs. Desulfobacteraceae use sulfur compounds as their main energy source and are found in diverse habitats but are mostly observed in saline and hyper saline waters and in polar ice in Antarctica. Additionally, several Antarctic and halophilic prokaryotes also contain this putative MetE sequence (e.g., Granulosicocuss and Pseudorhodobacter species).

\subsection{Discussion}

The potential for $\mathrm{B}_{12}$ to define ecological niches in marine microbial communities is of great importance in seasonally variable environments like Antarctic seas. In Antarctic coastal waters, the marine microbial community has a higher potential for $\mathrm{B}_{12^{-}}$ limitation than other oceanographic regions due to several factors: constant sunlight and increased UV radiation in the summer time that enhances photo-degradation of $\mathrm{B}_{12}$ (Carlucci et al., 1969), and a temporal decoupling between phytoplankton and bacterioplankton growth may lead to $\mathrm{B}_{12}$ co-limitation with iron (Bertrand et al., 2011a, 2015; Panzeca et al., 2006). While iron and $\mathrm{B}_{12}$ co-limitation has been observed in Antarctic seas, it is conceivable that the ecosystem could be tipped into primary $\mathrm{B}_{12}$ limitation if iron-replete growth exceeds bacterioplankton production and $\mathrm{B}_{12}$ supply. For example, this scenario may be possible in an early springtime bloom when there is a winter reserve of iron but seasonally low bacterial production and dissolved organic carbon (Ducklow et al., 2001), or in a summertime phytoplankton community dominated by $P$. antarctica colonies that are not efficiently grazed, reducing organic carbon supply to bacterioplankton, and thereby limiting the potential supply of $\mathrm{B}_{12}$ 
to auxotrophic co-occurring diatoms.

In this environment, the benefit of maintaining both MetH and MetE may outweigh the cost and infer a competitive advantage. A survey of MetE distribution among diatoms found that species from the Southern Ocean were significantly more likely to contain this $\mathrm{B}_{12}$-independent protein isoform, suggesting a biogeographical basis to retain MetE (Ellis et al., 2017). An analysis of Southern Ocean diatom MetE in transcriptomes revealed that $62 \%$ of the surveyed species contain MetE, in contrast to $11 \%$ of diatoms containing MetE in other oceanographic regions (Ellis et al., 2017). For example, the Southern Ocean diatoms Fragilariopsis cylindrus and Fragilariopsis kerguelensis have both MetE and MetH and can grow in the absence of $\mathrm{B}_{12}$ (Ellis et al., 2017). Furthermore, a recent examination of horizontal gene transfer (HGT) in diatoms identified five HGT enzymes from bacteria involved in the final steps of cobalamin synthesis in $F$. cylindrus, potentially inferring a competitive advantage to salvage cobalamin degradation products (Vancaester et al., 2020). Some marine protists, including P. tricornutum and E. huxleyi can remodel cobalamin via modification of the molecule's lower ligand, using CobT, CobS, and CobC (Helliwell, 2017). It is notable that genes and proteins involved in the synthesis of the corrin ring, final $\mathrm{B}_{12}$ synthesis, and activation and synthesis of DMB were not observed in P. antarctica cultures. Only the CobW protein, of hitherto unknown function though possibly involved in coordinating the cobalt atom in the corrin ring, was found to be significantly different between -/ $+\mathrm{B}_{12}$ conditions. Other polar phytoplankton species would be under similar environmental selection pressures to maintain MetE and cobalamin salvage pathways.

This study confirms that $P$. antarctica CCMP 1871 flexibly regulates its metabolism in response to $\mathrm{B}_{12}$ availability via a trade-off between MetH and the putative MetE. In culture, the same phytoplankton strain displayed markedly different morphotype ratios under low and high iron conditions, and also responded differently to the tested range of $\mathrm{B}_{12}$ concentrations. That single cells under low iron conditions respond to lower $\mathrm{B}_{12}$ concentrations and switch to MetH at $1 \mathrm{pM}$, may infer an advantage for using scarce cobalamin supplies in the early Antarctic spring; while high iron conditions and colonies may require a larger concentration of $100 \mathrm{pM} \mathrm{B}_{12}$ from bacterioplankton production to switch to MetH. Even though a higher $\mathrm{B}_{12}$ concentration may be needed to switch to MetH in colonial cells, a benefit could be increased growth rate, as seen in the cultures with replete Fe and $\mathrm{B}_{12}$ (Fig.2-2). In either case, maintaining both MetE and MetH enables a flexible approach to scarce, fluctuating $\mathrm{B}_{12}$ concentrations in the environment - a key advantage for $P$. antarctica over $\mathrm{B}_{12}$-auxotrophic diatoms. Furthermore each morphotype's ecophysiology could be uniquely "tuned" to $\mathrm{B}_{12}$ concentrations expected during their succession (early spring for solitary cells and spring and summer for colonial). This result motivates future work to examine if $P$. antarctica colonies can acquire $100 \mathrm{pM} \mathrm{B} \mathrm{B}_{12}$ from their environment or if this concentration necessitates the direct supply from associated bacterioplankton. Detecting MetH and MetE in field metaproteomes is challenging due to its relatively low abundance in cells, but it still remains an important biomarker for $\mathrm{B}_{12}$-stress and may help us understand the metabolic relationship between $P$. antarctica colonies and their natural microbiomes. 
$P$. antarctica colonies are documented to have bacteria growing within phycosphere and also within the colony, and have been proposed as a potential $\mathrm{B}_{12}$-source for growing colonies. For example, Delmont et al. (2014) report a near 1:1 ratio of $P$. antarctica algal cells and SAR92 bacterial cells coexisting inside colonies. However, SAR92 does not synthesize $B_{12}$, and it was concluded that their interaction does not depend on the exchange of the vitamin (Delmont et al., 2015). Our findings support that $\mathrm{B}_{12}$-based symbioses are not necessary for $P$. antarctica, although perhaps under Fe-replete conditions additional $\mathrm{B}_{12}$ and a switch to MetH may provide a boost to growing colonies.

\section{Why maintain and express MetE even under $B_{12}$-replete conditions?}

A possible underlying biochemical need for MetE in P. antarctica is for the assembly and maintenance of flagella throughout their life cycle stages. Eukaryotic flagella (and closely related cilia) are organelles that play critical roles in motility, sensory perception, and life cycle development. Chlamydomonas reinhardtii is a biflagellate green alga that maintains both MetE and MetH. In C. reinhardtii, MetE is a confirmed flagellar protein (found in the axoneme, outer doublets, and throughout); furthermore, MetE is actively up-regulated in gametes during the conversion of vegetative cells and in regenerating flagella (Kurvari et al., 1995; Pazour et al., 2005; Schneider et al., 2008; Sloboda and Howard, 2009). Curiously MetH is not found in the flagellar proteome (Pazour et al., 2005). C. reinhardtii preferentially expresses MetH over MetE when $B_{12}$ is available. MetE is thought to be needed to produce SAM (regardless of exogenous $\mathrm{B}_{12}$ concentrations) for methylation involved in posttranslational protein modifications, as evidence of MetK also present in the flagellar proteome along with other methyltransferases (Pazour et al., 2005). Due to flagella (and cilia) being resorbed prior to cell division, it has been suggested that MetE, MetK, and SAM are required for protein methylation during the assembly/disassembly of flagella, thus playing an important role in cell cycle regulation (Bloodgood, 1974; Schneider et al., 2008). In the case of limited $B_{12}$, it would be expected that cells increase MetE and MetK to produce methionine and SAM; this is observed in P. antarctica $-\mathrm{B}_{12}$ conditions and a MetK/AMS1 isoform (contig_37275_134_436__) is most highly expressed in the purely flagellate cell cultures. Although the flagellar MetE characterization has only been done in $C$. reinhardtii, this study serves as an appropriate reference point to compare proteomic changes observed here in $P$. antarctica and in other flagellated eukaryotes.

Given the potential use of MetE in the flagellated life cycle stage and for the overall ability to methylate proteins and nuclear histones to regulate metabolism, the ability to maintain and regulate both MetE and MetH would infer a metabolic flexibility to $P$. antarctica under rapidly changing ecological conditions and throughout its complex life cycle. Furthermore, due to the life cycle transitions, both flagellated single cells and non-motile vegetative cells would have a need to tightly regulate MetE and MetH in response to its environment, regardless of ambient $\mathrm{B}_{12}$ supply. Protein methylation is an essential process, especially for histone methylation for chromatin structure and transcriptional control in the nucleus to adjust cellular metabolism to environmental 
conditions and life cycle stages. Indeed, in the MA-plots - which compare the log abundance ratios (M-values) versus log averages (A-values) between two sample treatments - (Figs.2-9) and scatter plots (Figs.2-7 and 2-8) histones were among the most abundant and highest fold-change proteins in this study between $-/+\mathrm{B}_{12}$ conditions, suggesting the fine-tuning of genetic material to control the life cycle and metabolism of $P$. antarctica. The cell division, flagellar proteins, and histone changes suggest that $\mathrm{B}_{12}$ can influence cell cycle regulation. Interestingly, while many marine photosynthetic eukaryotic algae have flagella or cilia, these organelles are absent from red algae (Rhodophyta) and pennate diatoms (Daugbjerg and Guillou, 2001). These two groups are absent in the phylogenetic tree of putative MetE - note that the pennate diatoms with canonical MetE were added to the tree to inspect their relationship. The putative MetE found in P. antarctica is distantly homologous to the canonical MetE from pennate diatoms (Fragilariopsis kerguelensis, Fragilariopsis cylindrus, Grammonema cf. islandica, Fistulifera solaris, and Phaeodactylum tricornutum) which instead is more similar to MetEs from species belonging to Chromalevolata, Rhodohyta, Chlorophyta, and Cyanobacteria (Helliwell et al., 2011). These findings suggest that Phaeocystis antarctica, and perhaps other haptophytes and flagellated eukaryotes, maintain a flexible $\mathrm{B}_{12}$ metabolism to respond to cobalamin scarcity and changes in their polymorphic life cycles.

\subsection{Conclusion}

Examining the micronutrient-sensitive ecophysiology of keystone phytoplankton species is necessary to understand the biogeochemical consequences of changes in Antarctic seasonal seas. However, most eukaryotic phytoplankton have large, complex genomes that have yet to be sequenced due to challenges in genome assembly, annotation, and other bioinformatic analyses. This study used a complementary proteomic and transcriptomic approach to examine the physiological response of cultures of axenic $P$. antarctica strain CCMP 1871 to multifactor iron-B ${ }_{12}$ stress conditions. We confirm that iron plays a key role in the cell cycle of $P$. antarctica, as evidence by the ratio of single cells versus colonial cell in Fig.2-1 (Bender et al., 2018).

This work revealed that $P$. antarctica is not a $\mathrm{B}_{12}$-auxotroph, as recently suggested (Nef et al., 2019). Rather P. antarctica regulates its methionine synthase isoforms MetH and MetE according to $\mathrm{B}_{12}$ availability and has a flexible $\mathrm{B}_{12}$-ecophysiology. Two putative MetE proteins were identified in the protein abundance data and used to reexamine the transcriptomic assembly, ultimately resulting in an in silico construct of an open reading frame (ORF) that contains PP-binding, MetE, and pyridine nucleotide-disulphide oxireductase conserved domains. This construct was then searched in available $P$. antarctica strain transcriptomes, MMETSP, and NCBI-nr databases and was found to be present in a wide range of marine organisms. Notably, this ORF was found in several eukaryotes, including other P. antarctica strains, several haptophytes, dinoflagellates, and chlorophytes. The putative MetE protein was also found to be related to MetE proteins in bacteria and archaea. Furthermore, the putative MetE identified in this study is homologous but distantly related to the 
canonical MetE found in pennate diatoms. The phylogenetic tree of this putative MetE returned matches to eukaryotes that curiously have flagellated cells and many of which have complex, polymorphic life cycles. We suggest that this putative MetE may be involved in the flagellar proteome and may consequently be an important part of the life cycle, as observed in $C$. reinhardtii. This use may highlight a reason for $P$. antarctica to maintain both MetE and MetH, regardless of $\mathrm{B}_{12}$ availability. This flexibility is an advantage in the highly seasonal environments of coastal Antarctic seas, known to be limited by both iron and $\mathrm{B}_{12}$ compared to other oceanographic regions. Indeed, the escape from $\mathrm{B}_{12}$-limitation may enable $P$. antarctica to bloom early in the austral spring when glacial melt rates and irradiance are rising, bacterioplankton populations are low, and $\mathrm{B}_{12}$ is scarce enough to limit $\mathrm{B}_{12}$-auxotrophic diatoms. Furthermore, their multiple morphotypes may infer a fitness advantage whereby solitary cells can out-compete other phytoplankton due to their low iron requirement.

Furthermore, the findings in this study provide a significant contribution to the understanding of $\mathrm{B}_{12}$-specific protein and gene regulation in $P$. antarctica, with wide applicability to other marine phytoplankton species. Although eukaryotic algae primarily use $\mathrm{B}_{12}$ in a core set of enzymes, this study confirms that cobalamin and its metabolic by products via the methionine cycle, influence the overall metabolism of $P$. antarctica via DNA, RNA, and protein regulation. Future work includes further quantification and structural characterization of the putative $\mathrm{B}_{12}$-independent methionine synthase isoform identified in this study. The methionine synthase activity of this putative MetE protein can be tested via complementation in a $\mathrm{B}_{12^{-} \text {auxotrophic }}$ MetE knock-out, perhaps in Phaeodactylum tricornutum (Bertrand et al., 2007). The protein sequence, peptides, and MS spectra and chromatograms of the putative MetE can be potential biomarkers for field-based metaproteomic studies to investigate the $\mathrm{B}_{12}$-condition of $P$. antarctica cells in situ. 


\subsection{Supplemental Materials}

\subsubsection{Growth curves}

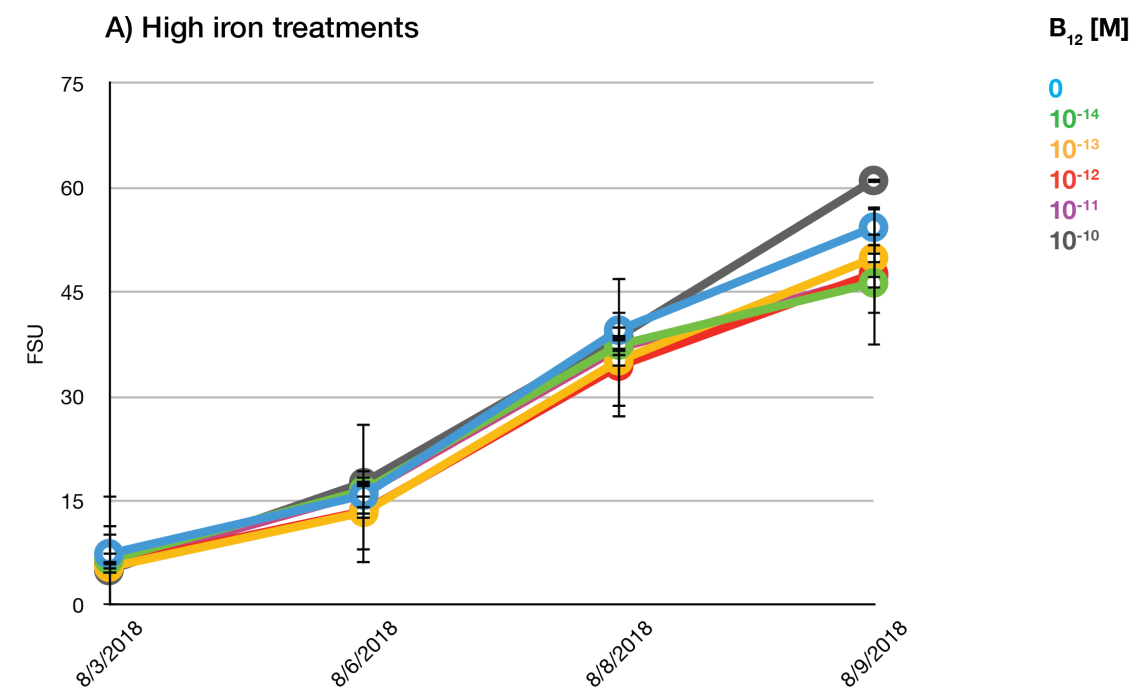

B) Low iron treatments

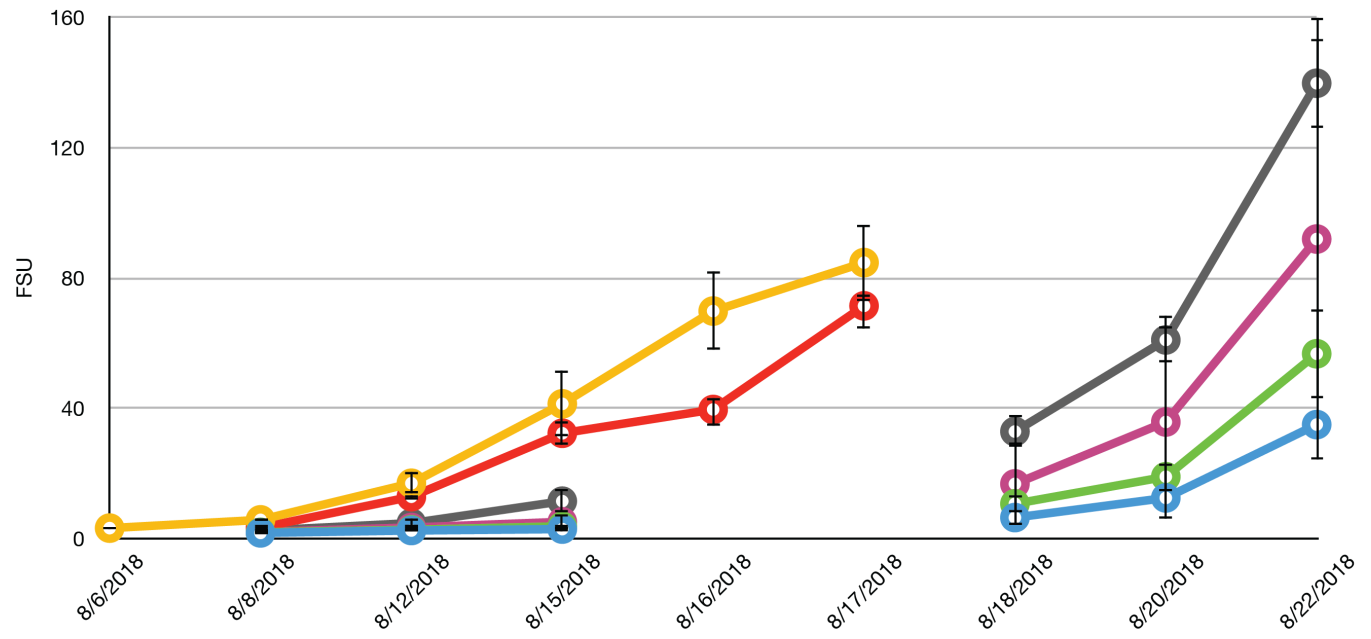

Figure 2-13: Growth curves of $P$. antarctica strain CCMP 1871 in a range of $\mathrm{B}_{12}$ treatments under A) high iron and B) low iron conditions. Fluoresence (FSU) over time is plotted as the mean of biological replicates $(n=3)$ with errorbars representing the standard deviation. Cells were harvested at the last plotted time point, either in mid- or late-exponential growth phase. Cultures were acclimated for several transfers $(>10)$ over a one year period. 


\subsubsection{Protein sequences for MetH and MetE contigs}

The protein sequences and contig number identifiers of the MetH and MetE proteins are presented below from the translated transcriptomic assembly. The putative MetE sequence is in Fig.2-16. The two MetE contigs found in P. antarctica strain CCMP 1871 (contig_21595_2_1159__ and contig_16786_147_2921_-) are 386 and 924 amino acids in length, respectively. A pairwise alignment (using BlastP on NCBI) returns an E-value of 1.5 with $15 \%$ coverage and $29.67 \%$ identity, indicating that these two sequences are not similar. When a multiple sequence alignment is conducted using BlastP on NCBI, with the reference sequence being the putative MetE sequence (CCMP1871_in_silico_edited), then contig_21595_2_1159_- covers $29 \%$ of the query with $100 \%$ identity, and contig_16786_147_2921_- covers $67 \%$ of the query with $96.35 \%$ identity. These results clarify that both MetE contigs, when reexamined in the transcriptomic assembly, were aligned onto a single open reading frame belonging to the putative MetE sequence.

\section{MetH Contig}

$>$ contig_51544_3_2534_+

KFLNMLIPEPDISKLPLMIDSSKFHIVEAGLKCCQGKCIVNSISLKEGEEEFVKKAKLVKRYGAAVVVMAFDEVG QAAGLDDKIRICKRAYDIMVGPRVGFPPHDIIFDPNVLTIATGLSEHDNYGKDFIEASRWITTNLPGAKISGGVS NLSFGFRGLTALREAIHAVFLYHAIQAGMTMGIVNAGAMPIYEDIEQPMRNYVEEVVLNYSEDGGHVERLLKFAE EEKERREAAGPNAPKVVDKKEWRNKPVGERLTHALVKGMAEF IDEDTEEARHLFPTGLEVIEGPLMAGMNVVGDL FGAGKMFLPQVIKSARVMKKAVAYLLPYMEEEKAAAAKLRADAGLPPITAKGRGVVVMATVKGDVHDIGKNIVGV VLGCNNYKVVDVGVMCNARDILQAVKDNDADILGCSGLITPSLDEMVTVAKEMERTGLTVPLLIGGATTSKMHTA VKVFPNYPSGFAMHVLDASRAVSVCESLLNDKKRVDFQADVKEQYEEMREDHYASLASRKFITLEKARAKMLPVE WKANVPMKPKLLGTKTFNDYPLAELLPYIDWNPFFQVWQLRGKYPNRGYPQIFKDETVGVEAKKLHDDALALIDE VVQKKMLTAKGVVGIWPANAVGDDIQVYSADGSGKHEGTFHTLRQQEEREDSVYYALSDFVAPKDSGVADYIGAF AVSCGFGCEAVCAQLRAENDDYKGIMMEAVADRLAEAFAELLHVKMRTELWGYAPDEKLSCEDLLKTKYTGIRPA PGYPTQPDHTEKNLMWKLLDAEKATGIELTDSLAMLPAASVSALVFGNPCSTYFQAGKLCKDQVTDYAKRKGMTI KEVEKWMGPYLGYDDEAQ

\section{MetE Contigs}

$>$ contig_21595_2_1159_-

MSTASKKAISDVWKACAEISGADPASQPDSDTSLFDMGLDSLGLAELVIQLEEVYGEGSLTVDDILAAPTLGEVA SCLPGAAAAPAPVKAAPVKAAPSVVVATKPAAKPTPRHPSSSTPKNAPPPMYKTATPARKPTPVAPKPTPVAATP RTPANGAAQAIIERLAKLENESRELRSLVGSLAVGSTPVPMAPMSSAVPEADDIQVEMCYEEGCEAPPSAEDNWI RTTHVGSLPRAAKGESGDIMEIVRMQLACGIDI INDGEWSRDNYIADLLSRIDGLGSADGKAGVALPMSSSCMCE MPLATDMGDVPTYAQRFTGGNGLITLNPARVAKADTACYARPQYKHSSIDALRNSLQPFLHSLRAAGRHPSQAFW SVPSPGTLAVF

$>$ contig_16786_147_2921__

VAHVRGERHLAHARGGHGEGHAGLAVGGAEAVDAREQVFGGDHTAYVQALARAVAPEYETIAATGMQLQVDCPDL AMGRHTRHSHLTDEEFCDVAAANVEALNLALRLANVPPEQVRYHVCWGNYAGPHHHDVDAASLWPIMANVQAKYV 
VLEAANPRHCHEVVLFEKAVQCGHFRADQVIVPGLIDTTAARVEHPKVICERLLRFVRAVGHPSRVIASTDCGFA STARSVAITHDLAWRKLHALVEGARLATATLMDMNAPVPLAGPGLTPTSFRAAILVCSKSKSAAASALTAACEHL HPALARRASTIDIIGLDMSQPPAAQAAQTAAALKWAVDAPIALVAVGNGAADVAAQTAAILAADKSCSRRDTRVF DALALGAASSPEGAADDIVREMAQVRGSAGFDKRRLVMGRNPVPLPEQTEVVVIGAGLLGLMAAARLKAAGRSVI ILEKRSVVAGIWSLFANSHSQVNSSEGGYCLKDLLPPAARAKATQNRDHSTAAEILEDAAALAHHLRDCIYTEVG VVKVLGQDGDYQVIAEESNPVGGTGSTRVISASGVVLAINDRVGLPRPLSCPGMEDFKAAGGNVADGTADALNHV KWQGKKVVIFGCGAFAIENVRTALEAGAAHVTVVARRLGTICPKI IDYLNFVKPWDAEYRHDTATNVKQLQAWRK LYKESTATTPTCWPGKIKHDGHTISVSDVWFIGHHLGKMSTMVGHLDHLTEGQVHLSDGEVLEADVVVGCIGFER NTTFCEQLTGRTVVHHSNYLDKNMMYLADAEIDETAFNSFFGSSVVEYAKFFSNVFVEGFNRPDELGEYLWGDNV PITNVKERKWTQYIAVANTLIANDPIMANHVSTQVSLRTEHFYRTMPPASFVAVNKKEWQELHTRLNGGVPVLPD AQLPYFFDDAASWCAPADEILLKN

\subsubsection{Heme biosynthesis pathway is differentially abundant in response to $B_{12}$}

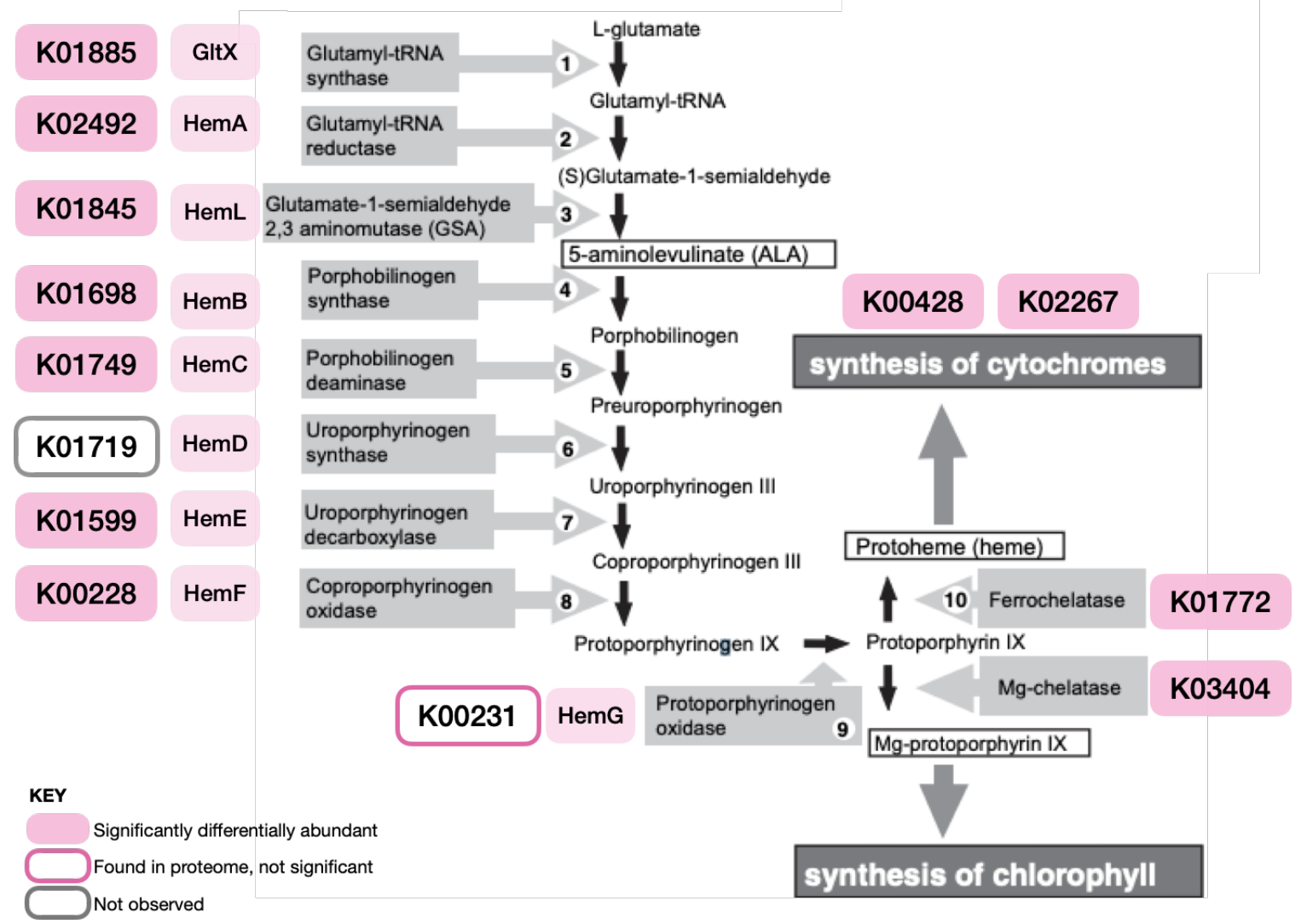

Figure 2-14: Nearly all proteins involved in heme biosynthesis were observed to be significantly differentially abundant in response to $\mathrm{B}_{12}$ (PLGEM, $\left.P<0.05\right)$. Proteins are identified with their KEGG annotation the color key indicates whether or not they were found in the proteome and if they were significantly different. The base figure of heme biosynthesis is from (Oborník and Green, 2005) and modified to represent the data from this study. 


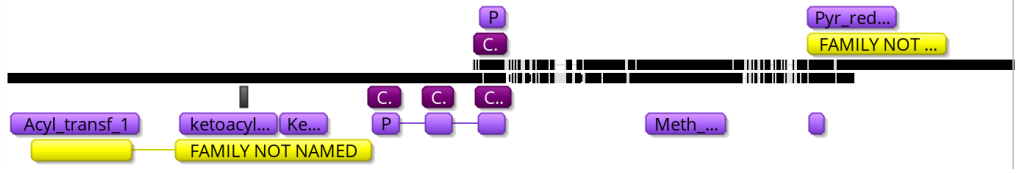

Figure 2-15: Domain arrangement diagram indicating the putative $\mathrm{B}_{12}$-independent methionine synthase (MetE) protein domain in Phaeocystis antarctica species (A) and the closest protein phylogenetic match Scyphosphaera apsteinii (B), for comparison. The query was an in silico construct based on an alignment with the putative MetE contigs identified via proteomics and then examined in the transcriptomic assembly and annotated using InterPro. Conserved domains on the $P$. antarctica in silico sequence include a phosphopantethein attachment site (PP-binding, a prosthetic group that acts as a "swinging arm" for the attachment of amino-acid groups or fatty acids), $\mathrm{B}_{12}$-independent methionine synthase (MetE), and pyridine nucleotide-disulphide oxioreductase (Pyr_redox_2). The query match of $S$. apsteinii also includes further N-terminal conserved domains, acyl transferase (AcpP) and ketoacyl asynthase (KS).

>CCMP1871_in_silico_edited

MSTASKKAISDVWKACAEISGADPASQPDSDTSLFDMGLDSLGLAELVIQLEEVYGEGSLTVDDILAA PTLGEVASCLPGAAAAPAPVKAAPVKAAPSVVVATKPAAKPTPRHPSSSTPKNAPPPMYKTATPARK PTPVAPKPTPVAATPRTPANGAAQAIIERLAKLENESRELRSLVGSLAVGSTPVPMAPMSSAVPEAD DIQVEMCYEEGCEAPPSAEDNWIRTTHVGSLPRAAKGESGDIMEIVRMQLACGIDIINDGEWSRDN YIADLLSRIDGLGSADGKAGVALPMSSSCMCEMPLATDMGDVPTYAQRFTGGNGLITLNPARVAKA DTACYARPQYKHSSIDALRNSLQPFLHSLRAAGRHPSQAFWSVPSPGTLAVFCEDRVFGGDHTAYV QALARAVAPEYETIAATGMQLQVDCPDLAMGRHTRHSHLTDEEFCDVAAANVEALNLALRLANVPP EQVRYHVCWGNYAGPHHHDVDAASLWPIMANVQAKYVVLEAANPRHCHEVVLFEKAVQCGHFRA DQVIVPGLIDTTAARVEHPKVICERLLRFVRAVGHPSRVIASTDCGFASTARSVAITHDLAWRKLHALV EGARLATATLMDMNAPVPLAGPGLTPTSFRAAILVCXXXXXXXXXXX $X X X X X X X X X X X X X X X X R A S T I D I I G$ LDMSQPPAAQAAQTAAALKWAVDAPIALVAVGNGAADVAAQTAAILAADKSCSRRDTRVFDALALG AASSPEGAADDIVREMAQVRGSAGFDKRRLVMGRNPVPLPEQTEVVVIGAGLLGLMAAARLKAAG RSVIILEKRSVVAGIWSLFANSHSQVNSSEGGYCLKDLLPPAARAKATQNRDHSTAAEILEDAAALAH HLRDCIYTEVGVVKVLGQDGDYQVIAEESNPVGGTGSTRVISASGVVLAINDRVGLPRPLSCPGMED FKAAGGNVADGTADALNHVKWQGKKVVIFGCGAFAIENVRTALEAGAAHVTVVARRLGTICPKIIDYL NFVKPWDAEYRHDTATNVKQLQAWRKLYKESTATTPTCWPGKIKHDGHTISVSDVWFIGHHLGKMS TMVGHLDHLTEGQVHLSDGEVLEADVVVGCIGFERNTTFCEQLTGRTVVHHSNYLDKNMMYLADAE IDETAFNSFFGSSVVEYAKFFSNVFVEGFNRPDELGEYLWGDNVPITNVKERKWTQYIAVANTLIAND PIMANHVSTQVSLRTEHFYRTMPPASFVGQQEGVAGAAPPPSTAACPCCPMPSSRTSSTTPRAGA RRRTRSSSRTEQRQSREEQSGPGLLYGGVRWQECHSRWTSK*

Figure 2-16: Protein sequence of in silico putative MetE construct. 


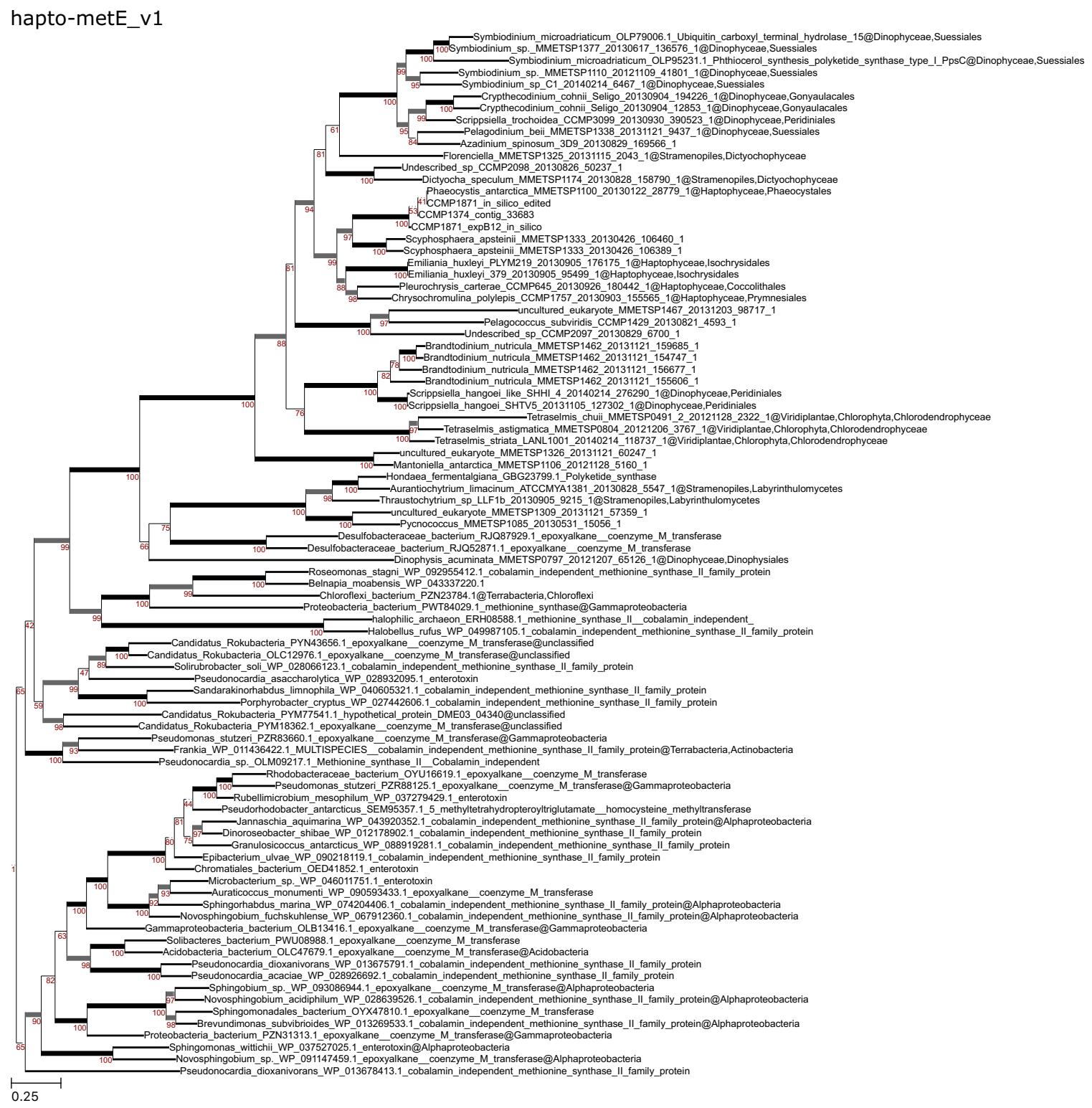

Figure 2-17: A phylogenetic tree (maximum likelihood consensus tree) of the putative MetE protein searched against NCBI-nr, MMETSP, and assemblies of the haptophytes C. tobinii and E. huxleyi from JGI. This putative MetE protein sequence is found across domains and in a range of eukaryotes that have flagella and polymorphic life cycles. The scale bar represents 0.25 amino acid substitutions per site. The maximum likelihood bootstrap values are indicated at the branches. The dotted line in the Phaeocystis clade denotes an interrupted branch of short length that was adjusted to fit in the figure. Tree made in collaboration with Z. Fussy. 


\section{Chapter 3}

\section{Patterns of microbial community driven cobalt and cobalamin uptake in Antarctic seas}

"... and these are unicellular pieces of slime that live by the million in pools on very nearly every ice floe all over this endless sea of ice, which we like to call a place of death! Mother Earth has a strange ability to produce life everywhere. Even thus ice is fertile ground to her."

- Fridtjof Nansen (1893-1896) on the Fram, recording his thoughts after observing protists living in Arctic sea ice (Bell, 2012).

Context: The data presented in this chapter were collected as part of the CICLOPS research cruise (Cobalamin and Iron Co-Limitation of Phytoplankton Species, NBP1801) aboard the U.S. $R / V$ Nathaniel B. Palmer from December 2017 to February 2018. As a participant during the cruise, I helped to plan experiments; collect, filter, and process trace metal clean seawater samples; organize, monitor, and sample the on board amendment-incubation experiments; and measure radiochemical cobalt and cobalamin uptake rates. The chemotaxonomic pigment data discussed here is provided by Nicole Schanke and Peter Lee from the lab of CICLOPS chief scientist Giocommo DiTullio; and the cobalt species concentrations were measured by Rebecca Chimel as part of the Saito Lab team aboard the cruise. These collaborators have kindly shared this data with me for this study. Dr. Mak Saito has given many thoughtful comments on the contents of this chapter and generously welcomed me into his lab to conduct the cobalt and cobalamin uptake measurements both at WHOI and at sea in Antarctica. 


\section{Abstract}

A full understanding of the biogeochemical cycling of cobalt and cobalamin (vitamin $\mathrm{B}_{12}$ ) in Antarctic coastal seas is limited by the lack of in situ measurements of micronutrient uptake into the marine microbial community. In this study, the ${ }^{57} \mathrm{Co}$ radiotracer incubation method was applied to determine the Co and $\mathrm{B}_{12}$ uptake rates across 15 sites during the Cobalamin and Iron Co-Limitation of Phytoplankton Species (CICLOPS) cruise from the Amundsen Sea to Ross Sea and Terra Nova Bay, Antarctica from December 2017 to February 2018. Concurrent uptake rates of ${ }^{57} \mathrm{CoCl}_{2}$ and ${ }^{57} \mathrm{~B}_{12}$ were measured on $0.2 \mu \mathrm{m}$ and $3 \mu \mathrm{m}$ filters to measure the total community and phytoplankton community uptake, respectively. This dataset was integrated with hydrographic, chemical (including total and labile cobalt), and chemotaxonomic phytoplankton pigment data to explain the observed patterns in ${ }^{57} \mathrm{Co}$ and ${ }^{57} \mathrm{~B}_{12}$ uptake in the context of Antarctic phytoplankton bloom dynamics. Data were analyzed in three spatio-temporal scales: (1) a week-long transect from the Amundsen Sea to Terra Nova Bay, (2) by geographic region (Amundsen Sea, Ross Sea, and Terra Nova Bay), and (3) by individual stations. Across all sites, ${ }^{57} \mathrm{Co}$ and ${ }^{57} \mathrm{~B}_{12}$ uptake rates were highest at the surface and decreased with depth and were dominated by the $\geq 3 \mu \mathrm{m}$ size fraction. Minimal ${ }^{57} \mathrm{~B}_{12}$ and ${ }^{57} \mathrm{Co}$ uptake was observed below $200 \mathrm{~m}$; the latter is consistent with the observed lack of manganese oxidizing bacteria that are typically a dominant pathways for dCo removal via co-oxidation and scavenging in other oceanographic regions. Potential uptake rates (also called \% uptake rates), which were the percent of radiotracer added that was assimilated, are reported for ${ }^{57} \mathrm{Co}$ and ${ }^{57} \mathrm{~B}_{12}$. Total community potential ${ }^{57} \mathrm{Co}$ uptake had a mean of $4.42 \%$ and ranged from $0.04 \%$ to $26.46 \%$ ( $n=76$ ), while potential ${ }^{57} \mathrm{~B}_{12}$ uptake had a mean of $17.77 \%$ and ranged from $0.1 \%$ to $94.16 \%(n=107)$. Cobalt uptake rates were calculated for samples where total Co concentration was also measured, revealing a mean of $0.61 \mathrm{pmol} \mathrm{L}^{-1} \mathrm{~d}^{-1}$ and range of $0.01 \mathrm{pmol} \mathrm{L}^{-1} \mathrm{~d}^{-1}$ to $3.06 \mathrm{pmol} \mathrm{L}^{-1} \mathrm{~d}^{-1}(n=53)$. These relatively high potential ${ }^{57} \mathrm{Co}$ uptake rates indicate that both $\mathrm{Co}$ and $\mathrm{B}_{12}$ are important, scarce resources that are rapidly cycled within the ecosystem. Both cobalt and $\mathrm{B}_{12}$ uptake were correlated with fresher, warmer surface waters, suggesting higher uptake and, consequently, greater proposensity for limitation in meltwater regions. Further, while these micronutrients are related, they are cycled differently through members of the marine microbial community. A strong correlation was found between uptake rates and chemotaxonomic pigments that reveal diatom-driven ${ }^{57} \mathrm{Co}$ and ${ }^{57} \mathrm{~B}_{12}$ uptake, even in a thriving Phaeocystis antarctica bloom in the Amundsen Sea. These results confirm the nutrient-like dynamics of Co in Antarctic seas and support prior observations of

diatom-dominated $\mathrm{Co}$ and $\mathrm{B}_{12}$ cycling. The high potential uptake of ${ }^{57} \mathrm{Co}$ and especially ${ }^{57} \mathrm{~B}_{12}$ suggest that Antarctic diatoms (and other phytoplankton groups) are under vitamin stress, and potentially higher cobalt and $\mathrm{B}_{12}$ stress than previously observed in this region. To date, this study presents the largest collection of potential ${ }^{57} \mathrm{Co}$, ${ }^{57} \mathrm{~B}_{12}$, and Co uptake uptake rates from Antarctic coastal waters. These measurements help to constrain the fluxes of $\mathrm{Co}$ and $\mathrm{B}_{12}$ assimilated into biomass and enable a more thorough comparison of the related biogeochemical cycles and their microbial drivers. 


\subsection{Introduction}

The coastal seas of Antarctica are critical components of Earth's climate system. The Ross Sea and Amundsen Sea sustain some of the most expansive phytoplankton blooms in the Southern Ocean and play an important role in the global carbon cycle via the biological pump (Arrigo et al., 1999). The regions' seasonally high rates of primary productivity have direct feedbacks on local ocean biogeochemistry, carbon export, and ecosystem dynamics (Arrigo et al., 2008). The marine environment bears the signature of the phytoplankton community composition, which influences the capacity for carbon export and ecology due to the distinct ecophysiologies of the dominant bloom-forming phytoplankton types: diatoms and the colonial haptophyte Phaeocystis antarctica. A typical seasonal succession is as follows: solitary cells then colonies of P. antarctica bloom in the early spring and summer, followed by an increase in bacterioplankton, and then the growth of a variety of diatoms in the summer through autumn (typically Fragilariopsis and Pseudonitzschia species) (Arrigo et al., 1999; DiTullio and Smith, 1996; Ducklow et al., 2001; Smith et al., 2003). In Antarctic systems, P. antarctica has only been observed to bloom in its colonial life stage and not the unicellular, flagellated morphotype (Schoemann et al., 2005). Recent lab culture experiments provide compelling evidence that natural $P$. antarctica may transition into colonies given sufficient iron supply (Bender et al., 2018) and the results of Chapter2. However, the controls of seasonal succession in the phytoplankton community remain debated.

The extreme seasonality of these Antarctic ecosystems means that primary production is controlled by physical factors like irradiance, with constant sunlight at the peak of austral summer and darkness in the winter. Importantly, when the sun does shine in these high nutrient, low chlorophyll (HNLC) ecosystems, marine primary production is limited by the availability of micronutrients, especially iron (Fe) and cobalamin (vitamin $\mathrm{B}_{12}$ ) (Bertrand et al., 2007; Moore et al., 2013). Trace metal and vitamin concentrations are low and highly variable across the global ocean, but perhaps most variable in extreme Antarctic habitats. It is well established that light and micronutrient limitation can control primary productivity in Antarctic waters (De Baar et al., 2005; Martin, 1990; Sedwick et al., 2000). The more recent insight that "keystone micronutrients" like $\mathrm{B}_{12}$ can control community composition has major implications for local biogeochemistry and ecosystem structure (Bertrand et al., 2007). For example, P. antarctica assimilates twice as much $\mathrm{CO}_{2}$ per mole of phosphate compared to polar diatoms (Arrigo et al., 1999); is a driver of the climatically active gas dimethyl sulfide (DMS) that affects ocean-atmosphere dynamics and sulfur nutrient cycling; and is minimally grazed by microzooplankton in its large colonial morphotype (Caron et al., 2000). A more complete understanding of the potential for $\mathrm{B}_{12}$ limitation, and by association the vitamin's metal co-factor cobalt (Co), and the overall biogeochemical cycling of these micronutrients is limited by the lack of in situ measurements of uptake into the marine microbial community.

In this study, ${ }^{57}$ Co radiotracer incubation experiment data is presented from the Amundsen Sea to the Ross Sea (from December 2017 - February 2018) that is integrated with hydrographic, chemical (total and labile Co), and phytoplankton chemotaxonomic pigment data to examine the microbe-driven flow of $\mathrm{Co}$ and $\mathrm{B}_{12}$ in 
Antarctic coastal ecosystems. The research goal was to gain insight into the concurrent ecological cycles of dissolved cobalt and cobalamin to constrain uptake rates in the context of community composition, hydrographic properties, and nutrient conditions. Using trace-metal clean (TM) techniques, the radio-labeled cyanocobalamin $\left({ }^{57} \mathrm{~B}_{12}\right)$ and cobalt chloride $\left({ }^{57} \mathrm{CoCl}_{2}\right)$ in situ uptake rates were measured for the marine microbial community collected on $\geq 3 \mu \mathrm{m}$ and $\geq 0.2 \mu \mathrm{m}$ pore-size filters, representing the eukaryotic phytoplankton and total plankton community size fraction, respectively. Few oceanographic studies of in situ $\mathrm{B}_{12}$ and Co uptake have been conducted (Bertrand et al., 2007, 2011a; Koch et al., 2012; Saito et al., 2010; Taylor and Sullivan, 2008). To date, this study is the largest set of concurrent cobalamin and cobalt uptake rate measurements, in terms of geographical range, time span, and sample number: with 15 stations through the Amundsen Sea and Ross Sea, 6 of which are full-depth, totaling over 100 samples in each of the two size-fractionated groups.

Over the past fifteen years, field expeditions to the Southern Ocean and Antarctic seas have conducted a series of nutrient amendment incubation experiments to test the micronutrient limitation status of natural seawater microbial communities. In the late austral summer of 1995, ambient iron concentration $(<0.2 \mathrm{nM})$ limited both $P$. antarctica and diatom species, except at the sea ice melt edge where a local diatom bloom had sufficient iron; the same study found that $>1 \mathrm{nM}$ iron was replete for the early summer P. antarctica bloom (Sedwick et al., 2000). Along the Antarctic Peninsula in 2005, the local phytoplankton community was co-limited by iron and $\mathrm{B}_{12}$, as observed by a 3 -fold increase in chlorophyll a with $+\mathrm{Fe}$ and $+\mathrm{B}_{12}$, compared to a 1.5-fold increase with + Fe alone (Panzeca et al., 2006). In the Ross Sea (austral summer 2005), iron addition spurred the growth of $P$. antarctica relative to diatoms; whereas the addition of $\mathrm{Fe}$ and $\mathrm{B}_{12}$ increased diatom growth of Pseudonitzschia subcurvata (Bertrand et al., 2007). The next year (austral spring 2006), a Ross Sea $P$. antarctica-dominated community was limited by $\mathrm{Fe}$ and not $\mathrm{B}_{12}$. This finding was consistent with a prior study in the area, where $\mathrm{B}_{12}$ was only limiting when bacterial abundance was low and vitamin additions did not stimulate the $P$. antarctica population (Bertrand et al., 2011a). In the late summer (mid-January) of 2013 and 2015, the McMurdo Sound microbial diatom-dominated community (which was predominantly Fragilariopsis cylindrus at the study site in the austral summer of 2013) at the sea ice edge was simultaneously limited by both iron and $\mathrm{B}_{12}$ (Bertrand et al., 2015). Advances over the past decade demonstrate that iron addition enhances bacterial growth, bacterioplankton are suppliers and significant sinks of $\mathrm{B}_{12}$, and that iron addition enhances $\mathrm{B}_{12}$ uptake in phytoplankton but not necessarily in bacteria (Bertrand et al., 2011a). Collectively, these field amendment experiments reveal a set of conditions for potential $\mathrm{B}_{12}$ limitation: regions of replete inorganic nutrients, sufficient bioactive trace metals, and low rates of bacterial production. Constraining the ecological cycling of $\mathrm{Co}$ and $\mathrm{B}_{12}$ through the marine microbial community remains challenging due to the high flux through the community and low-concentration in ambient seawater. The following analysis aims to shed light on the complex cycling of these micronutrients in relation to Antarctic microbial ecology. readers are directed to Appendix 3.A for a review of the coastal Antarctic ecological cobalt cycle and Appendix 3.B for the water mass characterization of samples collected during the 
CICLOPS research expedition.

\subsection{Materials \& Methods}

\section{Seawater sample collection}

During the CICLOPS cruise, a large survey of stations were sampled during the NBP-1801 expedition aboard the $R / V$ Nathaniel B. Palmer in the austral summer of December 2017 - February 2018. Seawater samples were collected using trace metal (TM) clean techniques with a TM sampling system that included a CTD (Conductivity, Temperature, and Depth), rosette with twelve Niskin bottles, and SeaBird equipment to measure temperature, salinity, dissolved oxygen, conductivity, and fluorometry. The TM sampling depths were determined based on the down-cast profile to select for interesting ecology- or hydrography-driven features. After the up-cast, Niskin bottles were handled with gloved hands and transported to the TM van, a positive-pressure TM-clean laboratory in a shipboard container for further processing. There, samples from the Niskin bottles were subsequently filtered through an acid-cleaned $0.2 \mu \mathrm{m}$ supor filter for dissolved Co measurements or directly dispensed into acid-washedrinsed with filtered TM-seawater, detergent, and acid $(0.1 \%$ citranox, $10 \% \mathrm{HCL}, \mathrm{pH}$ 2 filtered water) - polycarbonate bottles for radiotracer incubation measurements.

\section{Shipboard incubation}

Incubation bottles were transported in sealed containers from the TM van to the radiochemical van. The bottles were placed in a positive-pressure, TM environment constructed with a laminar flow hood and plastic sheeting to minimize trace-metal contamination. To the sample bottles, a tracer-level concentration of $0.1 \mathrm{pM}$ of both ${ }^{57} \mathrm{CoCl}_{2}$ and ${ }^{57} \mathrm{Co}-\mathrm{B}_{12}$ were added to separate incubation bottles. ${ }^{1}$ The bottles were sealed and placed into a flow-through, on-deck incubator with light screens that shaded the incubator to $20 \%$ percent ambient surface irradiance and allowed to incubate for 24 hours. The samples were then filtered onto $0.2 \mu \mathrm{m}$ and $3 \mu \mathrm{m}$ pore-size filters and preserved in petri dishes prior to counting.

Unlike other studies - e.g., Bertrand et al. (2007, 2011a) — cold (non-radioactive) cobalamin was not added, in an effort to not overestimate the uptake rates measured by this approach (Bertrand et al., 2007) that artificially increases the available $\mathrm{B}_{12}$ (an important consideration because luxury consumption is documented in many algal species (Droop, 1957, 2007). ${ }^{2}$ The limitation of this method is that without measured

\footnotetext{
${ }^{1}$ It is important to note that while $0.1 \mathrm{pM}$ was a best estimate to do a $\leq 10 \%$ tracer addition, it is possible that even this amount was larger than the standing stock of $\mathrm{B}_{12}$ in seawater in some stations.

${ }^{2}$ The method used in these studies to measure $\mathrm{B}_{12}$ uptake involved the addition of large amounts of $\mathrm{B}_{12}(40 \mathrm{pM})$ to facilitate the comparison of uptake rates between experimental samples without the need for measuring ambient $\mathrm{B}_{12}$ concentrations. Because calculated $\mathrm{B}_{12}$ uptake $=$ fraction of ${ }^{57} \mathrm{~B}_{12}$ taken up $\mathrm{x}$ total $\mathrm{B}_{12}$ concentration, these $40 \mathrm{pM}$ auditions significantly increased the calculated uptake rates compared to the standing stock of vitamin in seawater and do not represent in situ
} 
seawater $\mathrm{B}_{12}$ concentrations, only a percent ${ }^{57} \mathrm{~B}_{12}$ uptake rate can be calculated and upper and lower bounds placed on a range of uptake rates.

\section{Cobalamin and cobalt assimilation}

Radiotracer cobalamin and cobalt uptake rate was determined for 15 of stations, some of which included deeper profiles measurements up to $400 \mathrm{~m}$ for $\%{ }^{57} \mathrm{~B}_{12}$ uptake $(n=10)$ and 3 for $\%{ }^{57}$ Co uptake $(n=3)$ (Figs.3-21 and 3-22). ${ }^{3}$ The radiochemical samples were measured on a Canberra Germanium Gamma ( $\gamma$-ray) Detector, on board (GC2518) and on land (GL2020), until they were below $5.0 \%$ error for most samples, with higher errors for a few deeper samples with fewer counts $<10 \%$. The counts per minute at $122 \mathrm{keV}\left({ }^{57} \mathrm{Co}\right.$ peak) were corrected for decay between sample collection and measurements dates, a normalized to a \% uptake per day (accounting for the extra $0.1 \mathrm{pM}$ of radiotracer added), which was calculated by dividing the activity on each filter by the total activity added to each incubation bottle. For samples with measured cobalt concentrations, a Co uptake rate measurement was made, by multiplying the $\%$ uptake per day by the total dissolved cobalt concentration, representing an upper bound on the Co uptake rates, following methods in Saito et al. (2010). Previous studies (Bertrand et al., 2011b; Saito et al., 2010) demonstrated that glutaraldehydekilled control treatments had minuscule abiotic uptake (approximately $2-4 \%$ of live uptake) and that active $\mathrm{B}_{12}$ uptake measured was not significantly impacted by nonspecific adhesion to cells. Additionally, results from an experiment with a control and $\mathrm{NaN}_{3}$-poisoned samples of Antarctic bacterial isolates' uptake of ${ }^{57} \mathrm{Co}$ demonstrated that uptake is driven by actively growing and metabolizing cells (Taylor and Sullivan, 2008). Given that dead cells are a minor component of radiochemical uptake, this measurement was not conducted during this study.

\section{Phytoplankton pigment analysis}

The chemotaxonomic distribution of phytoplankton pigments was determined using HPLC as described in (DiTullio and Geesey, 2003). In the Southern Ocean, xanthophyll pigments are reliable chemotaxonomic indicators of phytoplankton group presence, specifically for diatoms and $P$. antarctica. The pigment analysis was conducted by $\mathrm{N}$. Shanke and G. DiTullio.

\section{Nutrient and micronutrient concentrations and cobalt speciation}

The samples collected for dissolved Co analysis were collected from a TM Niskin rosette and filtered with a $0.2 \mu \mathrm{m}$ Supor membrane filter (polyester sulphone, Pall Corporation, $142 \mathrm{~mm}$ diameter) in a TM van. The concentration and chemical speciation of cobalt

uptake rates, but rather that at a high $\mathrm{B}_{12}$ concentration (Fig. 7A in Bertrand et al. (2011a)).

${ }^{3}$ Actually, there are several profiles that extend even deeper $(600-700 \mathrm{~m})$. I want to include those, but need to find an appropriate place to discuss the very low uptakes at depth. 
in seawater was measured following methods described in Hawco and Saito (2018); Saito and Moffett (2001); Saito et al. (2010), to measure total and labile dissolved cobalt dCo. Briefly, cathod stripping voltammetry was used to measure dCo on the Metrohm $663 \mathrm{VA}$ and $\mu$ AutolabIII systems with a hanging mercury drop working electrode. Prior to voltammetric analysis, samples for total dCo were UV-irradiated for 1 hour on a Metrohm 705 UV Digester in order to liberate Co atoms from natural ligand-bound cobalt complexes; whereas labile dCo samples were not UV-irradiated, in order to preserve the weak-ligand bound Co compounds.

Labile cobalt refers to the cobalt fraction that is free $\left(\mathrm{Co}^{2+}\right)$ and easily exchangeable with weak natural ligands, and thereby measurable via ligand competition with a strong synthetic cobalt ligand, such as dimethylgloxime (DMG). Bound cobalt refers to cobalt fraction that is complexed to a very strong natural ligand of unknown structure. It is hypothesized that the strong natural ligand is likely $\mathrm{B}_{12}$ or one of its degradation products, wherein $\mathrm{Co}^{3+}$ is maintained in the corrin ring structure (Saito et al., 2002). Cobalt concentration and speciation measurements were conducted aboard the $R / V$ Nathaniel B. Palmer in a trace metal clean bubble and at WHOI in a Class- 100 clean room by R. Chimel. Major nutrients (nitrate + nitrite, phosphate, silicate, and ammonia) were analyzed using standard wet chemistry methods.

\section{Statistics, Seawater Properties, Plotting}

All statistical analysis were performed using python3 and stats.model (version 0.10.0). Neutral density was calculated following the PreTEOS-10 software in Matlab 2019a (Jackett and McDougall, 1997). Mixed layer depth was calculated using the python oceans "sw extras" package with the "zmld so" function for Southern Ocean waters developed by Mitchell and Holm-Hansen (1991). Figures were made using matplotlib (version 3.0.2) and Ocean Data View (version 4.7.10) with matplotlib or cmocean (version 2.0) colorschemes (Thyng et al., 2016).

\section{Data accessibility}

The data collected during CICLOPS (NBP-1801) is available via the Biological and Chemical Oceanography Data Management Office (BCO-DMO) on the project page.

\subsection{Results}

Presented next are the results of radiotracer incubation experiments that measured the uptake and assimilation of ${ }^{57} \mathrm{Co}$ into microbial biomass during the CICLOPS cruise (NBP-1801). On the research cruise, a total of 65 standard casts and 28 trace metal (TM) casts were collected. This study focuses on the TM casts and also uses hydrographic and phytoplankton chemotaxonomic data from the standard casts to contextualize observations. ${ }^{4}$ To further contextualize the uptake patterns, a water

\footnotetext{
${ }^{4}$ Upcast data was used from both the standard and TM CTD to correspond with water samples collected in Niskin bottles.
} 
mass characterization of Antarctic shelf waters was done for CICLOPS expedition samples (Fig.3-24a) in Appendix 3.B.

\subsubsection{Geographic context}

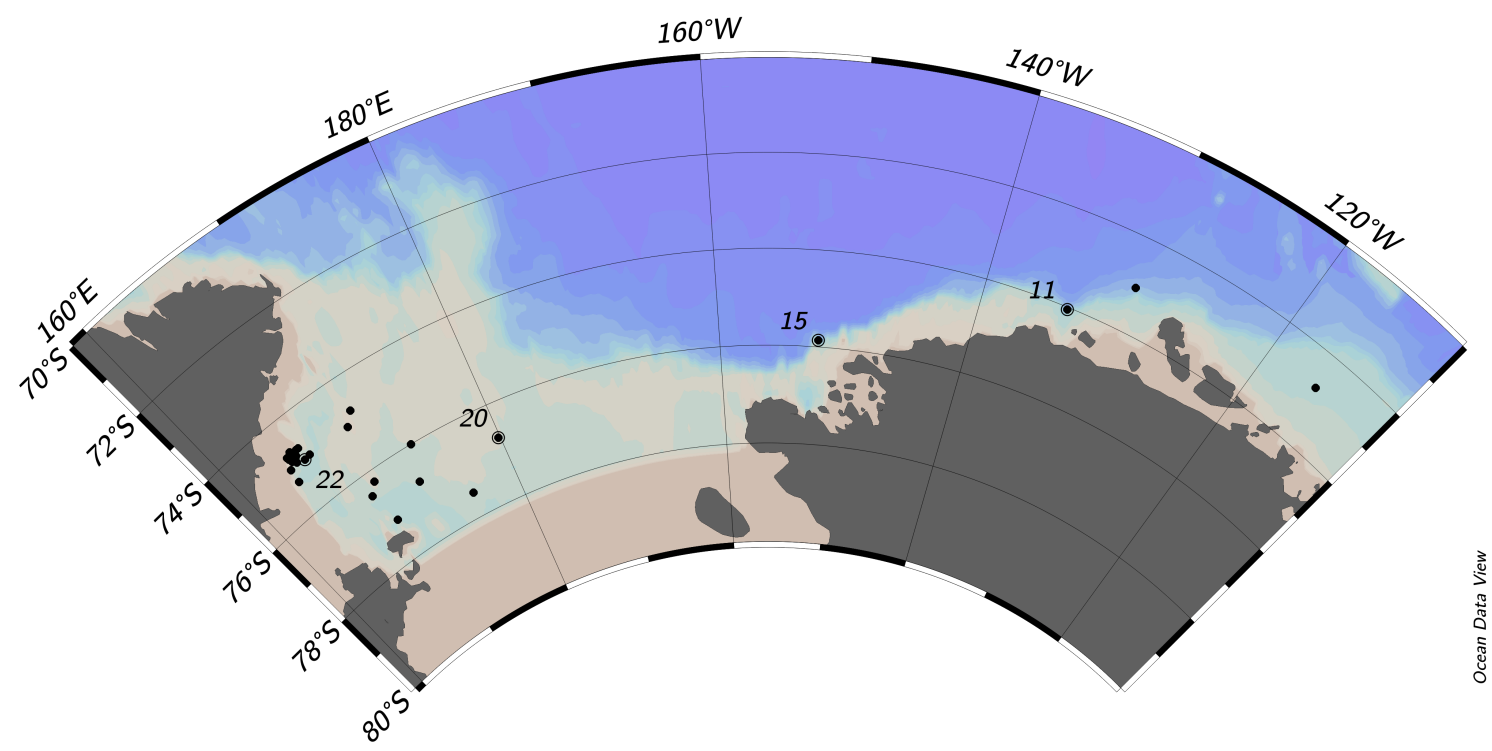

Figure 3-1: A map of the subset of CICLOPS trace metal cast stations where ${ }^{57} \mathrm{Co}$ and ${ }^{57} \mathrm{~B}_{12}$ uptake measurements were conducted. Highlighted with text are sites on the Amundsen-Ross Transect (Stations 11, 15, 20, 22). The P. antarctica bloom was in the Amundsen Sea region (TM Stations 11, 15). A mixed community was present in TM Station 20, and a diatom bloom was present in the Ross Sea (Station 22).

The CICLOPS cruise transited from Punta Arenas, Chile to the Amundsen Sea where coastal samples were collected on a westward path to the Ross Sea and ultimately Terra Nova Bay, Antarctica. Stations 1-18 were in the Amundsen Sea; and Stations 19-81 were in the Ross Sea, with a focus on Terra Nova Bay. Fig.3-20 is a station map of all CICLOPS sample sites. The following analysis is organized at three spatialtemporal scales: 1) a week-long transect from the Amundsen Sea to Terra Nova Bay (Fig.3-2), 2) by geographic region (Amundsen Sea, Ross Sea, and Terra Nova Bay), and 3) by individual stations. The Amundsen-Ross Transect is a unique dataset of four trace metal stations $(11,15,20,22)$ with hydrographic, chemical, phytoplankton pigment, and radiochemical uptake rate measurements (Fig.3-1).

\subsubsection{Hydrographic context: Water masses of the Amundsen Sea, Ross Sea, and Terra Nova Bay}

Figure 3-3 highlights the dominant Antarctic coastal water masses across the Amundsen Sea, Ross Sea, and Terra Nova Bay, following neutral density $\left(\gamma^{N}\right)$, potential temperature $(\theta)$, and salinity $(\mathrm{S})$ criteria highlighted in Fig.3-25 and Table 3-24b 


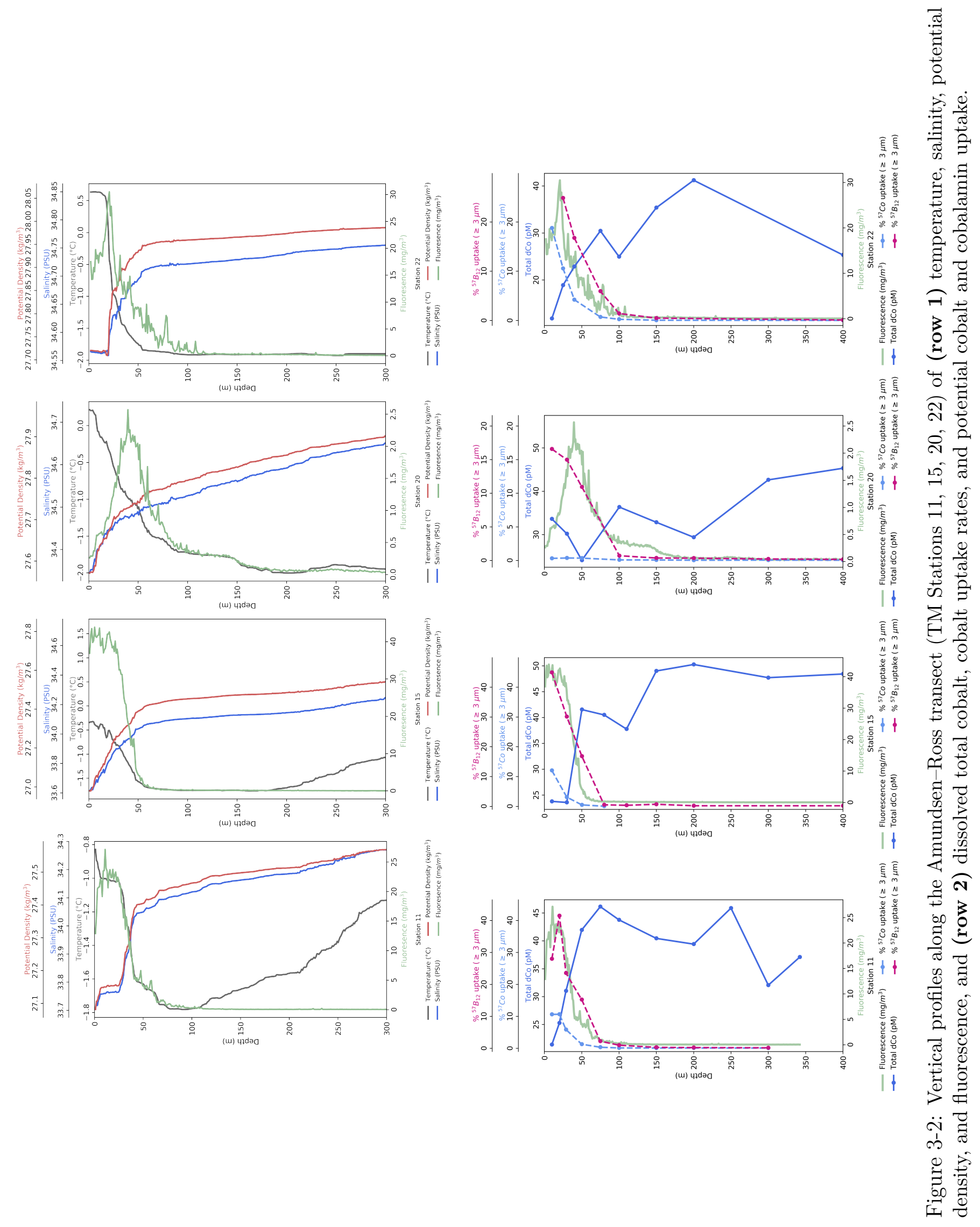


(Castagno et al., 2019; Jackett and McDougall, 1997; Mattson et al., 2012; Orsi and Wiederwohl, 2009). The interaction of these water masses influence the heterogeneous and complex Antarctic coastal surface seascapes in which seasonal phytoplankton blooms develop (Fig.3-26 shows the T-S diagram subset for all surface stations $\leq$ $200 \mathrm{~m}$ ). The characteristics of these water masses reflect the physical and chemical processes that modify nutrient availability for the marine microbial community, notably meltwater inputs from sea ice, glaciers, and ice shelves, which are important sources of bioessential trace metals (Noble et al., 2013; Planquette et al., 2013).

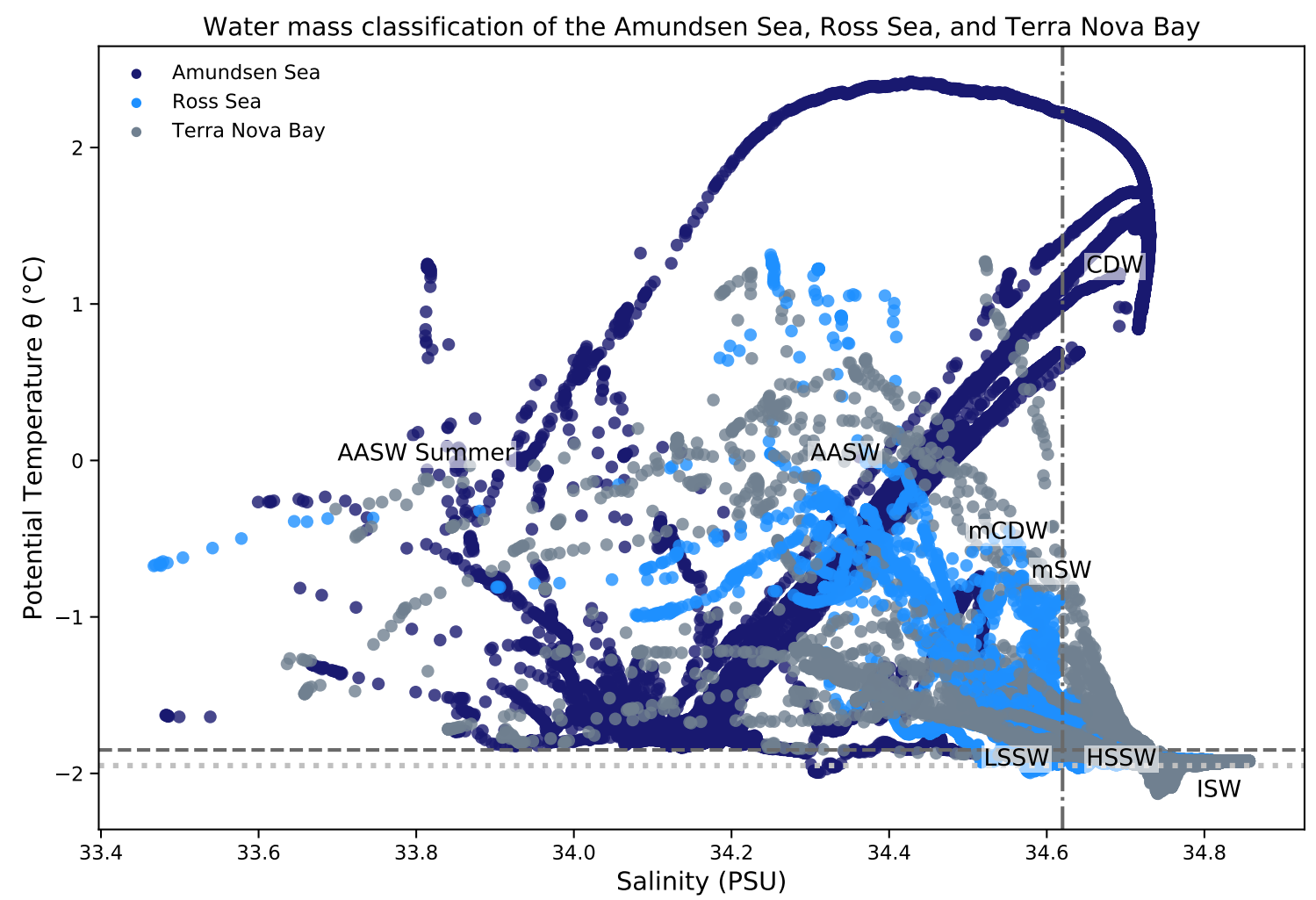

Figure 3-3: The Temperature-Salinity (T-S) relationships for the Amundsen Sea, Ross Sea, and Terra Nova Bay. The labels in the T-S diagram show the major water masses across these coastal Antarctic regions during the CICLOPS cruise (NBP 1801): modified Circumpolar Deep Water (mCDW/CDW), Antarctic Surface Water (AASW), modified Shelf Water (mSW/SW), Low and High Salinity Shelf Water (LSSH/HSSW), and Ice Shelf Water (ISW). The color of the data points correspond to geographic regions: Amundsen Sea (dark blue), Ross Sea (medium blue), and Terra Nova Bay (grey). Water masses are characterized according to neutral density, potential temperature, and salinity, following analysis in Castagno et al. (2019); Jackett and McDougall (1997); Mattson et al. (2012); Orsi and Wiederwohl (2009). The additional lines demarcate water mass properties: grey-dashed horizontal line is $\mathrm{mSW} / \mathrm{SW}$, white-dotted horizontal line is ISW/SW, and the grey-dash-dot vertical line is LSSW/HSSW. Readers are encouraged to examine the T-S diagram with neutral density values $\gamma^{N}$ in Fig.3-25 and Table 3-24b to view the water mass characterization criteria.

The Amundsen Sea is characterized by three primary water masses: Antarctic 
Surface Water (AASW), Circumpolar Deep Water (CDW), and modified Circumpolar Deep Water (mCDW), as defined by their neutral density values (Jackett and McDougall, 1997; Mattson et al., 2012). The surface waters are cold and fresh due to cooling and freezing in the winter and ice melt in the summer. The AASW Summer water has a distinctly lower salinity $(<34$ PSU) from melting sea ice and glacial meltwater inputs (Mattson et al., 2012). Often the AASW Summer mass forms a warmer, fresher surface layer of less than $50 \mathrm{~m}$ that overlies the remaining cold, fresh winter water (Talley, 2011). At depth, the relatively warm, saline CDW circulates over the Antarctic continental shelf, melting and eroding the basal Amundsen ice shelf. The convergence of these surface and deep waters result in a mixed water mass, the mCDW. The majority of samples on the continental slope of the Amundsen Sea had signatures of AASW, AASW Summer, and mCDW waters.

The Ross Sea is characterized by additional distinct water masses: Shelf Water (SW), modified Shelf Water (mSW), and Low and High Salinity Shelf Water (LSSW, HSSW), and Ice Shelf Water (ISW), along with mCDW and AASW (Mattson et al., 2012; Orsi and Wiederwohl, 2009). SW is near the freezing point, and so salinity becomes an important criteria to define water masses in this region (Talley, 2011). $\operatorname{HSSW}\left(\gamma^{N}>28.27 \mathrm{~kg} \mathrm{~m}^{-3}\right.$ and $\left.\theta<-1.85^{\circ} \mathrm{C}\right)$ is generated on the Ross Sea continental shelf by surface cooling, freezing, and brine rejection during sea-ice formation. As this dense, cold, briny seawater sinks down the continental slope, it mixes with CDW to result in Antarctic Bottom Water (AABW). Through a combination of increasing the solubility and biological pumps, HSSW and ISW in the Western Ross Sea mediates oceanic atmospheric $\mathrm{CO}_{2}$ uptake (Castagno et al., 2019). Terra Nova Bay, the "ice factory" of the Ross Sea, has a strong imprint of sea-ice formation and the HSSW and ISW, the latter is colder than $-1.95^{\circ} \mathrm{C}$ and reflects an interaction with the base of a nearby ice shelf (Orsi and Wiederwohl, 2009). The surface samples in the Ross Sea and Terra Nova Bay have signatures of AASW, AASW Summer, mCDW, mSW, and LSSW/HSSW.

\subsubsection{Community Context}

HPLC analysis of xanthophyll pigments are chemotaxonomic indicators of the presence of phytoplankton classes. The two most abundant accessory pigments across all stations - 19'-hexanolyxylfucoxanthin (19-Hex) and fucoxanthin (Fuco) - correspond to the region's dominant phytoplankton types: Phaeocystis antarctica and diatoms, respectively (DiTullio and Smith, 1996; Weber and Wettern, 1980). ${ }^{5}$ P. antarctica dominated surface waters in the coastal Amundsen Sea (TM Stations 10,11,15), while diatoms dominated in the Ross Sea (TM Stations 20,22). Stations 11-18 were dominated by $P$. antarctica $(\geq 80 \%)$ that contained pigment signatures indicative of high iron conditions, according to ChemTax analysis (DiTullio et al., personal communication, Nov 2019), Fig.3-4.It is important to note that fucoxanthin is a pigment found in many phytoplankton types. While it is often indicative of diatoms,

\footnotetext{
${ }^{5}$ Additionally, chlorophyll $c 3$ is a diagnostic pigment for Phaeocystis cell counts, and has been found to correlate to Phaeocystis biomass in other oceanographic regions (Breton et al., 2000; Muylaert et al., 2006).
} 
the chemotaxonomic analysis of Antarctic regions is more challenging to discern because $P$. antarctica also contains this pigment, though in a relatively smaller ratio. The ratio of 19-Hex:Fuco normalizes for the amount of Fuco in haptophytes like $P$. antarctica. In this region, other phytoplankton pigments were present in comparatively low concentrations, including peridinin and alloxanthin (indicative of dinoflagellates and cryptophytes). While Antarctic coastal waters are also home to a range of cryptophytes, dinoflagellates, and other nanoflagellate phytoplankton, the community is predominantly P. antarctica or diatoms (Petrou et al., 2016).
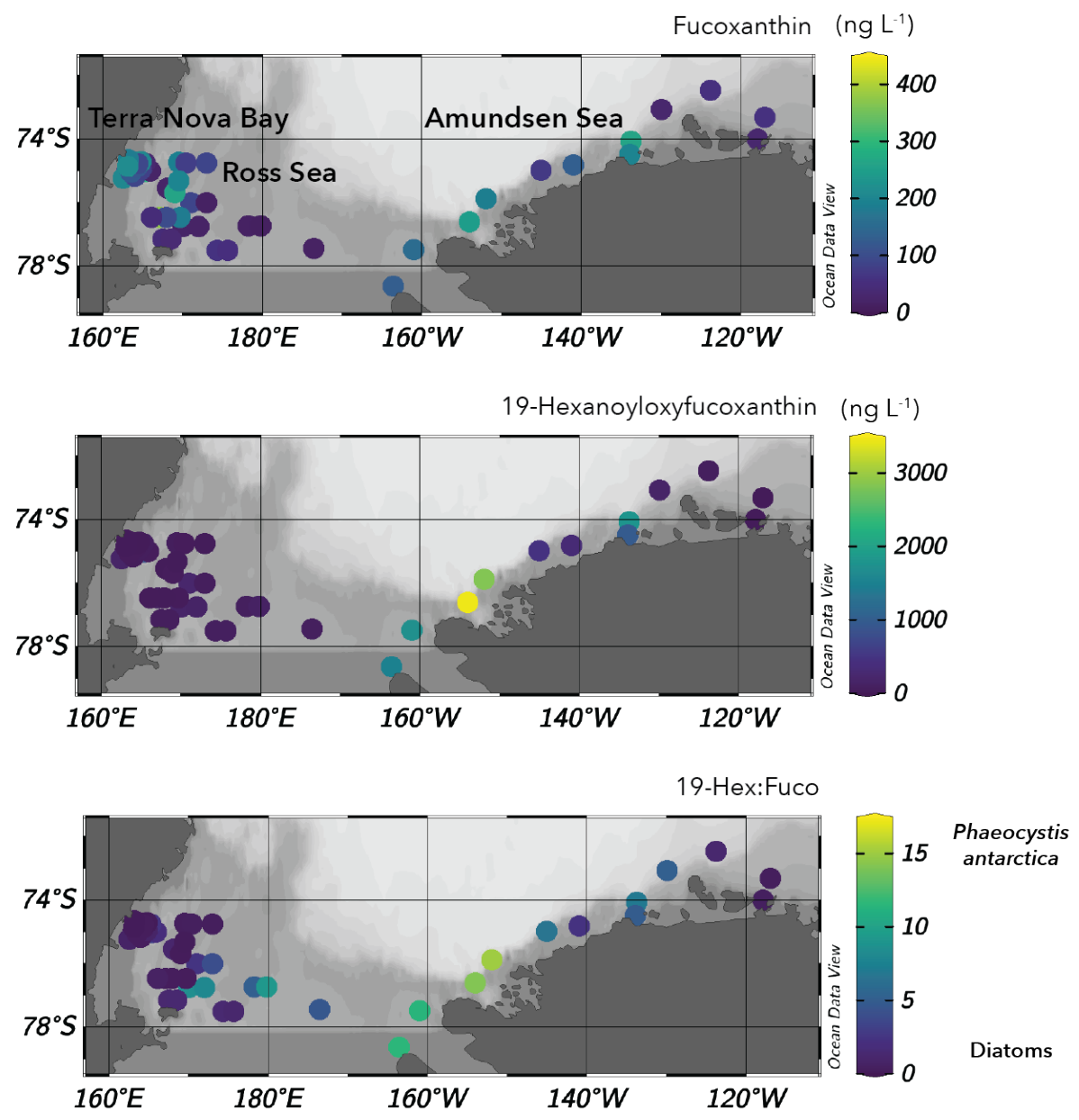

Figure 3-4: Phytoplankton chemotaxonomic pigment concentrations $\left(\mathrm{ng} \mathrm{L}^{-1}\right)$ during CICLOPS. Plotted are fucoxanthin (Fuco), 19'-hexanoyloxyfucoxanthin (19-Hex), and the ratio of 19-Hex:Fuco, which normalizes for the amount of Fuco found in haptophytes and indicates how dominant $P$. antarctica is relative to co-existing diatoms. The data represents pigment concentrations at the first depth, often $10 \mathrm{~m}$. Note the differences in the colorbar scales for the pigment data. P. antarctica pigments were $10 \times$ higher compared to diatom pigment concentrations. The figures were made using Ocean Data View. 


\subsubsection{Cobalt concentration and speciation}

The chemical speciation of dCo affects its bioavailability, with $\mathrm{Co}_{\text {labile }}$ being the most bioavailable form, especially for eukaryotic phytoplankton (Saito et al., 2002, 2004). $\mathrm{Co}_{\text {total }}$ had a nutrient-like profile across most stations, with minimum concentrations at the surface that increased with depth - mirroring patterns of ${ }^{57} \mathrm{Co}$ uptake, fluorescence, and phytoplankton pigments. Total dCo had a mean concentration of $37.4 \mathrm{pmol} \mathrm{L}^{-1}$ (maximum of $111.1 \mathrm{pmol} \mathrm{L}^{-1}, n=147$ ) across the cruise samples. Surface samples $(\leq 200 \mathrm{~m})$ had a lower total dCo mean concentration of $32.8 \mathrm{pmol} \mathrm{L}^{-1}$ (maximum of $52.3 \mathrm{pmol} \mathrm{L}-1, n=83)$, Table 3.3. The regional and surface vs. deep values of $\mathrm{Co}_{\text {total }}$, $\mathrm{Co}_{\text {labile }}$, and labile Co fraction are recorded in Table 3.4. Overall, $\mathrm{Co}_{\text {labile }}$ was a small fraction of $\mathrm{Co}_{\text {total }}$, ranging from $0 \%$ to $51.35 \%$ (mean of $10.9 \%, n=147$ ) across all samples measured in the Amundsen Sea, Ross Sea, and Terra Nova Bay. Furthermore, $\mathrm{Co}_{\text {labile }}$ was undetectable in most surface waters $(\geq 100 \mathrm{~m})$, likely driven by microbial consumption, as observed in the radiotracer incubation experiments. In the Amundsen Sea, labile dCo ranged from $0.0 \mathrm{pM}$ to $15.8 \mathrm{pM}$ (mean of $4.3, n=45$ ). Labile dCo ranged from from $9 \%$ to $41 \%$ of total Co along the AR transect (Stations 5, 10, 11, $15,20,22$, mean $=4.1 \%, n=67$ ). This indicates that the majority of dCo was bound to strong organic ligands of unknown structure and was less bioavailable to marine microbes.

Curiously, the results from CICLOPS are indicative of increased Co cycling and potential for limitation, compared to the CORSACS cruises from 2005-2006 (Saito et al., 2010). The results from all three cruises are summarized in Table 3.1. Comparison to measurements in the Ross Sea from CORSACS-1 (2006) and CORSACS2 (2007) suggests that $\mathrm{Co}_{\text {labile }}$ was previously more abundant in coastal Antarctic surface waters, whereas between December 2017-February 2018, most dCo was predominantly bound to strong organic ligands (Saito et al., 2010). Although CORSACS-2 samples were collected between November and December 2006, slightly earlier than the December to January sampling period, perhaps $\mathrm{Co}_{\text {labile }}$ was not depleted earlier in the austral summer. The natural seawater communities observed during CICLOPS had greater percent ${ }^{57} \mathrm{Co}$ uptake overall and less $\mathrm{Co}_{\text {labile }}$ and $\mathrm{Co}_{\text {total }}$ in the surface ocean. During CORSACS 2, Ross Sea surface waters had relatively high $\mathrm{Co}_{\text {labile }}$ and $\mathrm{Co}_{\text {total }}$ availability, with correspondingly less percent ${ }^{57} \mathrm{Co}$ uptake. Overall, the slight reduction in $\mathrm{Co}_{\text {total }}$ inventory and that most $\mathrm{dCo}$ is bound to strong organic ligands, indicates that it is less bioavailable to marine microbes in the surface ocean during the CICLOPS sampling period. ${ }^{6}$ These results suggest a possible regional change in the amount of total bioavailable Co, likely due to a combination of increased biological assimilation and changing supply to the surface ocean. Other factors may have also resulted in the observed changes between CICLOPS and CORSACS including differences in sampling periods, interannual variability, or the significantly larger sample number of this study; however, it remains important to consider the backdrop of climate change that is especially impacting the coastal Antarctic region when considering possible mechanisms.

\footnotetext{
${ }^{6}$ In the Discussion section, I speculate on the potential processes that could result in such a shift in the regional cobalt concentration.
} 


\begin{tabular}{|c|c|c|c|c|}
\hline Cruise & & CICLOPS & CORSACS $1^{\dagger}$ & CORSACS $2^{\dagger}$ \\
\hline Location & & Amundsen, Ross, TNB & Ross Sea, $n=21$ & Ross Sea, $n=16$ \\
\hline Timeframe & Unit & Dec 2017 - Feb 2018 & Dec 2005 - Jan 2006 & Nov 2006 - Dec 2006 \\
\hline Depth range & $\mathrm{m}$ & $(4-100)$ & $(10-75)$ & $(17-97)$ \\
\hline Labile Co & $\mathrm{pM}$ & $2.53(0.0-29.45), n=106$ & n.d. & 17.27 \\
\hline Total Co & $\mathrm{pM}$ & $29.96(0.0-54.25), n=61$ & 31.6 & 48.4 \\
\hline$\%{ }^{57} \mathrm{Co}$ uptake & $\% d^{-1}$ & $5.45(0.1-26.46), n=63$ & 2.81 & 0.55 \\
\hline Cobalt uptake & $\operatorname{pmol~L} \mathrm{L}^{-1} \mathrm{~d}^{-1}$ & $0.84(0.02-3.06), n=38$ & 0.67 & 0.25 \\
\hline
\end{tabular}

Table 3.1: ${ }^{\dagger}$ Comparison of dissolved cobalt concentrations and $\%{ }^{57} \mathrm{Co}$ from CICLOPS $(\leq 100 \mathrm{~m})$ and CORSACS 1 and 2 (Saito et al., 2010).

\subsection{5 ${ }^{57}$ Co and ${ }^{57} \mathrm{~B}_{12}$ uptake studies}

In Antarctic coastal waters, dCo is observed to have a nutrient-like rather than the typical hybrid-like profile, due to assimilation by the surface marine microbial community and the lack of scavening via co-oxidation of Co with manganese by bacteria at depth (Moffett and Ho, 1996; Saito et al., 2010; Taylor and Sullivan, 2008; Tebo et al., 1984). Active microbial assimilation into phytoplankton and bacterioplankton biomass is the dominant process removing dCo from seawater in Antarctic coastal ecosystems. The following results demonstrate the phytoplankton-driven uptake observed across the Amundsen Sea, Ross Sea, and in Terra Nova Bay, Antarctica.

\section{Co and ${ }^{57}$ Co uptake studies}

Figures 3-5 and 3-6 shows direct measurements of cobalt uptake rates made via ${ }^{57} \mathrm{CoCl}_{2}$ radiotracer incubation experiments on seawater samples from vertical profiles. The data plotted are the percent of radiochemical ${ }^{57} \mathrm{Co}$ added that was taken up onto filters, representing the amount of radiotracer assimilated into biomass, henceforth referred to as percent uptake (\% uptake). In order to infer the cobalt uptake rates ( pmol L ${ }^{-1} \mathrm{~d}^{-1}$ ), the measured uptake $\%{ }^{57} \mathrm{Co}$ uptake per day is multiplied by the total dissolved cobalt concentration $\left(\mathrm{pmol} \mathrm{L}^{-1}\right)$, following published methods (Saito et al., 2010). These cobalt uptake rates should be considered an upper bound, as they were calculated using total cobalt (labile + bound Co), and not labile cobalt alone, due to the extremely low values observed at sea (trace beyond detection limits, and thus unreliable for this uptake rate calculation). It should be noted that while labile cobalt is the most bioavailable, the bound cobalt fraction may be partially bioavailable to some marine microbes. The results discussed here assume that the radiotracers equilibrated with the entire dissolved Co pool over the course of the 24-hour incubation, but that there likely remain $\mathrm{Co}^{3+}$ complexes (e.g., vitamin $\mathrm{B}_{12}$ that did not fully equilibrate). Utilization of these different cobalt chemical species may also be determined by specific modes of transport in to the cell, which remains unknown for many marine microbes. Where cobalt concentrations are unavailable, the percent uptake remains a useful metric to consider the potential uptake of the natural community, which reflects the overall biological need for this trace metal. These uptake rate measurements can be interpreted as good approximations of the 
assimilation rate of $\mathrm{dCo}$ by marine microbes; specifically, the majority of $\geq 0.2 \mu \mathrm{m}$ size-fraction represents the assimilation of phytoplankton and bacterioplankton in Antarctic coastal waters and the $\geq 3 \mu \mathrm{m}$ size-fraction represents the phytoplankton community and any particle-associated bacterioplankton. A summary of $\%{ }^{57} \mathrm{Co}$ and ${ }^{57} \mathrm{~B}_{12}$ uptake and estimated Co uptake rates are presented in Table 3.2, and discussed below.

The spatial pattern of $\%{ }^{57} \mathrm{Co}$ uptake reflects the nutrient-like profile for Co in Antarctic coastal waters. Percent ${ }^{57} \mathrm{Co}$ uptake rates were highest in the upper $50 \mathrm{~m}$ and decreased with depth, indicating that phytoplankton and surface bacterioplankton uptake were more important removal mechanisms of surface Co, relative to bacterioplankton assimilation (or minimal scavenging and co-oxidation by manganese oxidizing bacteria) deeper in the water column, as seen in tropical and subtropical waters (Moffett and Ho, 1996; Saito et al., 2004). ${ }^{7}$ In Antarctic coastal systems, algal pigment concentrations are typically found in shallower depths, where we expect the highest rates of growth and nutrient uptake, as seen in the Co uptake profiles in Fig.3-5. The phytoplankton filter size fraction $(\geq 3 \mu \mathrm{m})$ contributes most to total community uptake $(\geq 0.2 \mu \mathrm{m})$, compared to the bacterioplankton size fraction. Measurements of cobalt uptake from the total community ( $\geq 0.2 \mu \mathrm{m}$ ) had a mean of $0.61 \mathrm{pmol} \mathrm{L} \mathrm{L}^{-1} \mathrm{~d}^{-1}$ and range of $0.01 \mathrm{pmol} \mathrm{L}-1 \mathrm{~d}^{-1}$ to $3.06 \mathrm{pmol} \mathrm{L}^{-1} \mathrm{~d}^{-1}(n=53)$. These uptake rates are comparable to Co uptake rates measured in the Ross Sea (maximum of $2.5 \mathrm{pmol} \mathrm{L}^{-1} \mathrm{~d}^{-1}$ ) during the CORSACS program (Saito et al., 2010). Whereas $\%{ }^{57} \mathrm{Co}$ uptake had a mean of $4.42 \%$ and range of $0.04 \% \mathrm{~d}^{-1}$ to $26.46 \% \mathrm{~d}^{-1}$ of ${ }^{57} \mathrm{CoCl}_{2}$ added $(n=76)$, again comparable to $\%{ }^{57}$ Co uptake $\mathrm{d}^{-1}$ rates measured during CORSACS-1 (mean $=2.8 \%$ ), with the notable difference of having higher mean and maximum $\%{ }^{57} \mathrm{Co}$ rates in this size fraction, especially compared to CORSACS-2 where the uptake rate values $\leq 1.06 \%$ (mean $=0.55 \%$ ), but total dCo concentrations were closer to $50 \mathrm{pM}$ (calculated from Table 3 of Saito et al. (2010)). The spatial trends observed with depth and between stations are similar for the total community $(\geq 0.2 \mu \mathrm{m})$ and phytoplankton community $(\geq 3 \mu \mathrm{m})$ size fractions, since phytoplankton are responsible for most of the uptake.

When all station data were separated by longitudinal bins for the Amundsen Sea, Ross Sea, and Terra Nova Bay, regional differences were found for the correlations between total dCo versus $\%{ }^{57} \mathrm{Co}$ uptake (Fig.3-7). Overall, there is a strong and significant correlation between $\mathrm{Co}_{\text {total }}$ and $\%{ }^{57} \mathrm{Co}$ uptake by the $\geq 0.2 \mu \mathrm{m}$ microbial community, except in the Ross Sea. Notably, $\%{ }^{57}$ Co uptake rates were low $(\leq$ $5 \%$ ) when total dCo was $>30 \mathrm{pM}$ and increased where total Co was minimal. For example, in Terra Nova Bay where total surface cobalt had the smallest average concentration ( mean $=26.3 \mathrm{pM}, n=32$ for samples $\leq 200 \mathrm{~m}$ ), and largest range from $0.0 \mathrm{pM}$ to $52.9 \mathrm{pM}$, the $\%{ }^{57} \mathrm{Co}$ uptake varied most strongly with dCo $\left(R^{2}=0.61\right.$, $p \leq 0.001, n=18)$. Interestingly, in Terra Nova Bay the average surface seawater temperature was lower than in the Amundsen Sea and Ross Sea, and correspondingly

\footnotetext{
${ }^{7}$ V. Oldham et al. (submitted for review May 2020) find undetectable Mn-oxidation at depth in some samples in the Ross Sea and Terra Nova Bay. It is worth noting that any deep uptake observed is then likely assimilation directly into bacteria and archaea, or sinking phytoplankton in this size class.
} 

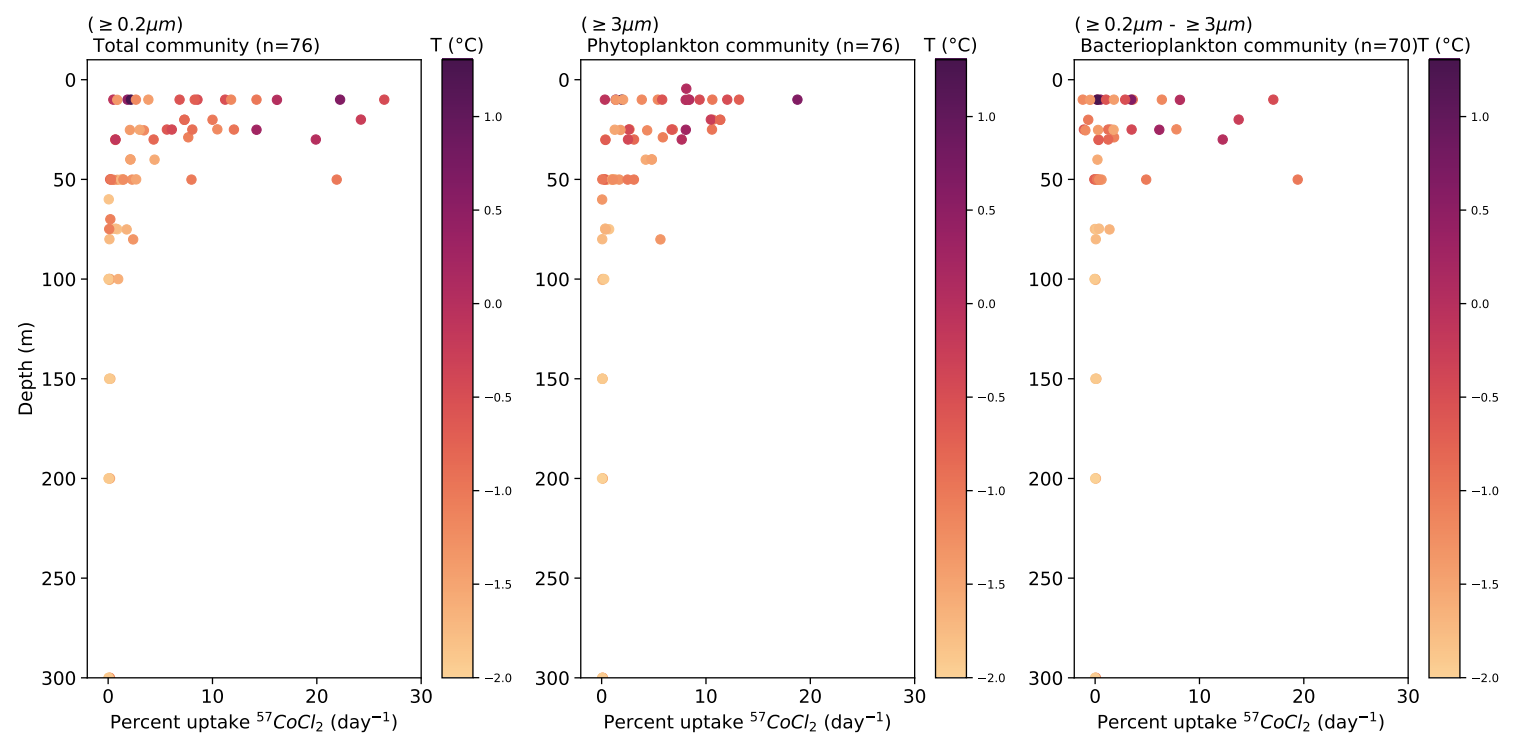

Figure 3-5: Surface percent ${ }^{57}$ Co uptake versus depth by size fraction, colored according to temperature $\left({ }^{\circ} \mathrm{C}\right)$. The bacterioplankton community is a proxy measured by subtracting the phytoplankton community uptake from the total community uptake. All plotted samples were counted to $<5 \%$ error on a Canberra Germanium Gamma Counter, so error bars are not included. Only the top $300 \mathrm{~m}$ of the data are shown.
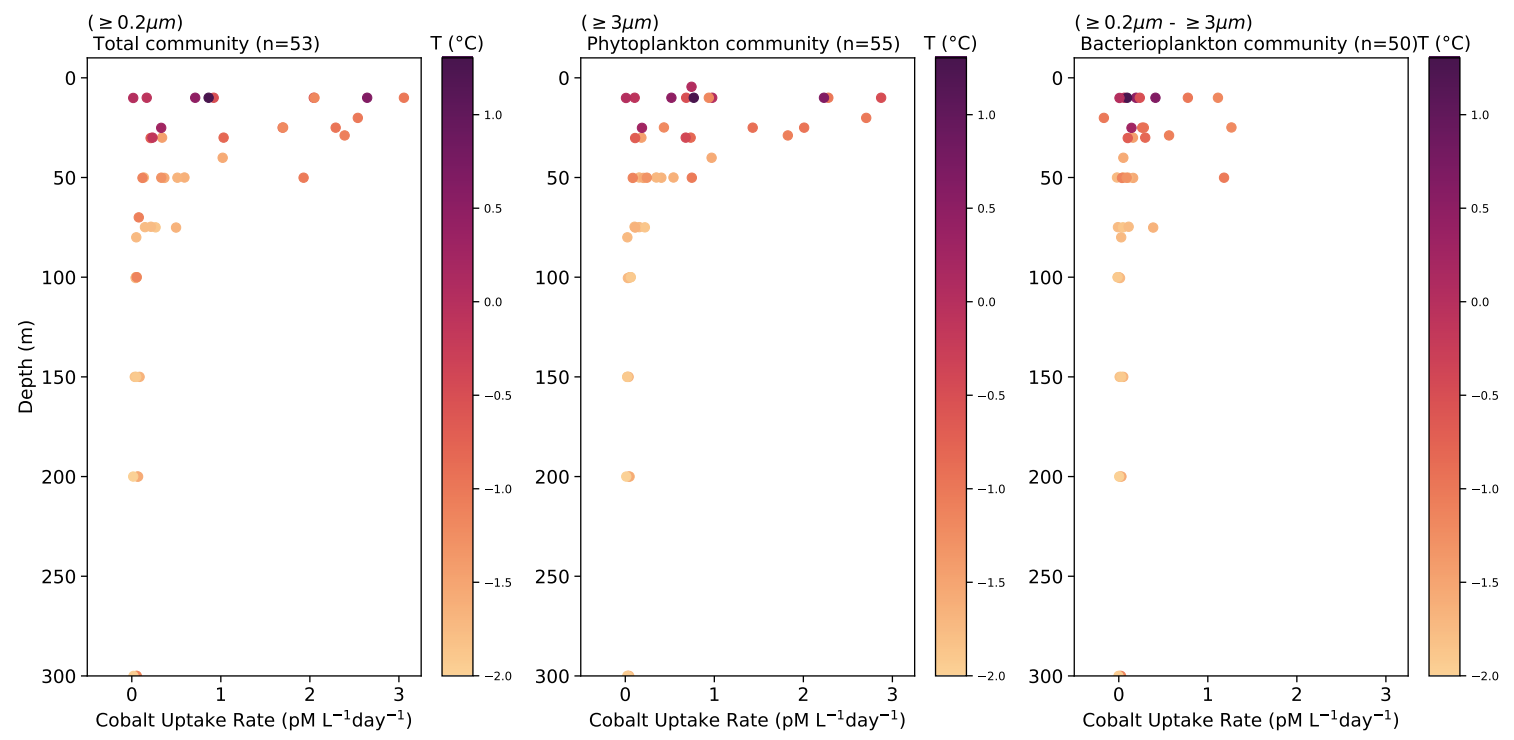

Figure 3-6: Surface cobalt uptake rate $\left(\mathrm{pML}^{-1} \mathrm{~d}^{-1}\right)$ versus depth by size fraction, colored according to temperature $\left({ }^{\circ} \mathrm{C}\right)$. Estimates of cobalt uptake rates are made by multiplying the $\%{ }^{57} \mathrm{Co}$ uptake by the total cobalt measured in these sample locations. The bacterioplankton community is a proxy measured by subtracting the phytoplankton community uptake from the total community uptake. All plotted samples were counted to $<5 \%$ error on a Canberra Germanium Gamma Counter, so error bars are not included. Only the top $300 \mathrm{~m}$ of the data are shown. 

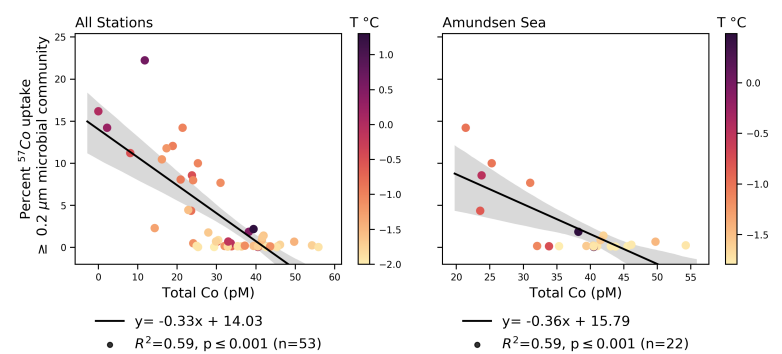

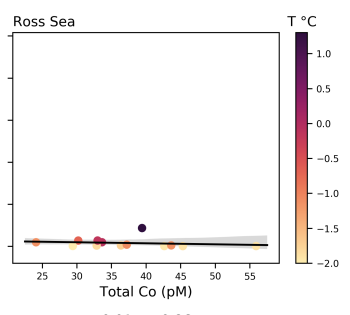

- $y=-0.01 x+0.86$

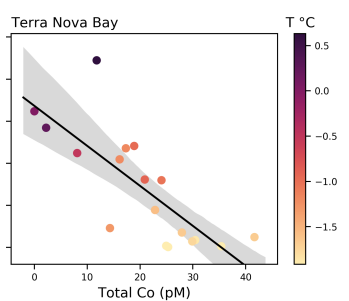

- $y=-0.48 x+16.82$

- $R^{2}=0.61, p \leq 0.001(n=18)$

Figure 3-7: There is a strong and significant correlation between total cobalt versus $\%{ }^{57} \mathrm{Co}$ uptake by the $\geq 0.2 \mu \mathrm{m}$ marine microbial community. This indicates that where ambient total cobalt concentrations are low, there is increased $\%{ }^{57} \mathrm{Co}$ uptake. The relationship is not significant in the Ross Sea region.

there is a temperature dependence with $\%{ }^{57} \mathrm{Co}$. The rates of $\%$ uptake increase with temperature, likely reflecting increased microbial metabolisms and growth in warmer waters.

\section{${ }^{57} \mathrm{~B}_{12}$ uptake studies}

Direct measurements of cobalamin uptake rates were made using radiotracer cyanocobalamin $\left({ }^{57} \mathrm{~B}_{12}\right)$ addition incubation experiments on vertical profiles. Percent ${ }^{57} \mathrm{~B}_{12}$ uptake rates were highest in surface waters (above $75 \mathrm{~m}$ ) and were driven by the phytoplankton community size fraction $(\geq 3 \mu \mathrm{m})$. The summary statistics for ${ }^{57} \mathrm{~B}_{12}$ uptake are in Table 3.2. The average ${ }^{5}{ }^{57} \mathrm{~B}_{12}$ uptake was $17.17 \%$ (0.1\% to $94.16 \%$, $n=107)$ for the $\geq 0.2 \mu \mathrm{m}$ community, $12.84 \%(0.06 \%$ to $75.89 \%, n=108)$ for the $\geq 3 \mu \mathrm{m}$ community, and $6.51 \%(0.0 \%$ to $39.67 \%, n=107)$ for the bacterioplankton community proxy. While phytoplankton dominate the $\%{ }^{57} \mathrm{~B}_{12}$ uptake in these samples, bacterioplankton are also substantial consumers of the radiotracer. Investing in $\mathrm{B}_{12}$ uptake proteins, even if a microbe can synthesize the vitamin, has an evolutionary advantage considering the metabolic and nutritional cost of synthesizing the trace vitamin.

In lieu of in situ cobalamin concentrations, potential uptake rates are estimated based on reasonable estimates of observed $B_{12}$ concentrations to facilitate the comparison across sites. Prior studies have measured $\mathrm{dB}_{12}$ concentrations in the range of $0.4 \mathrm{pM}$ to $4 \mathrm{pM}$ in the Gerlache Straight coastal waters off the Antarctic Peninsula, with surface dCo in the range of $25 \mathrm{pM}$ to $59 \mathrm{pM}$ (Panzeca et al., 2006). A profile of $\mathrm{B}_{12}$ (CN-, Me-, Ads-, and OH-) was measured under sea ice in McMurdo Sound, Antarctica (Tabersky, M.A. Thesis with M. Saito (2010)), and found well-mixed water under the

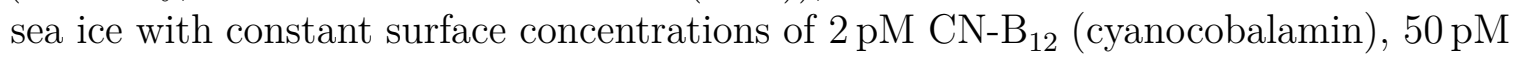

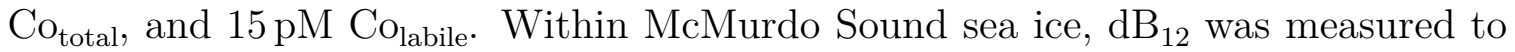
range from $6 \mathrm{pM}$ to $32 \mathrm{pM}$; however, seawater $\mathrm{dB}_{12}$ concentrations were only $0.5 \mathrm{pM}$ (Taylor and Sullivan, 2008). Finally, other $\mathrm{dB}_{12}$ concentrations varied between $\leq$ $2.4 \mathrm{pM}$ in the Indian sector of the Southern Ocean (Carlucci and Cuhel, 1977), 0.13 pM to $5 \mathrm{pM}$ in the North Atlantic (Panzeca et al., 2009), and $5 \mathrm{pM}$ to $87 \mathrm{pM}$ across a range of productive coastal waters (Sañudo-Wilhelmy et al., 2006). 

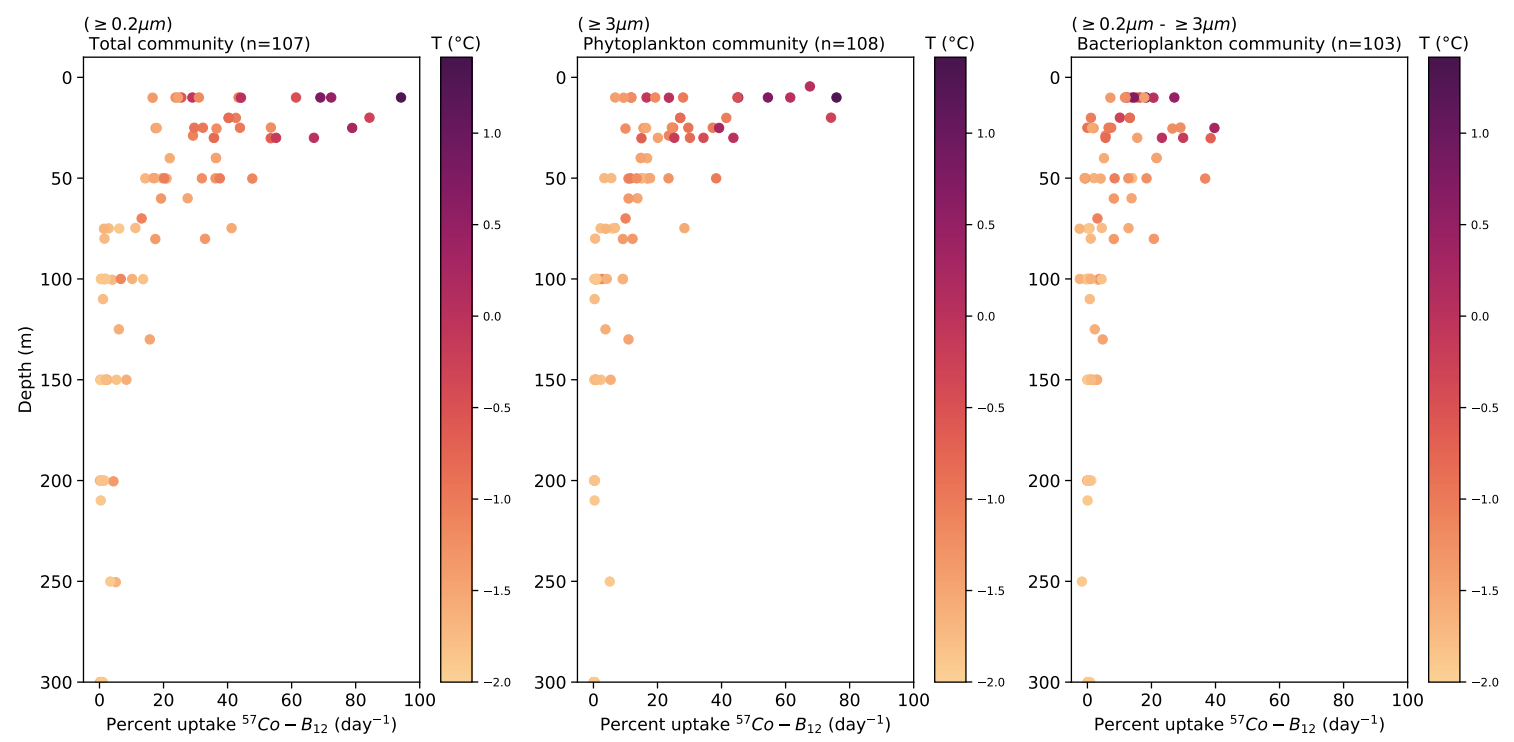

Figure 3-8: Surface percent ${ }^{57} \mathrm{~B}_{12}$ uptake versus depth by size fraction. Data are colored according to temperature $\left({ }^{\circ} \mathrm{C}\right)$. The bacterioplankton community is a proxy measured by subtracting the phytoplankton community uptake from the total community uptake. All plotted samples were counted to $<5 \%$ error on a Canberra Germanium Gamma Counter, consequently error bars are not included.

Assuming a consistent concentration of $1 \mathrm{pmol} \mathrm{L}^{-1} \mathrm{~B}_{12}$ (cyanocobalamin) throughout the water column, estimated $\mathrm{B}_{12}$ uptake rates (in pmol $\mathrm{L}^{-1} \mathrm{~d}^{-1}$ ) would range from 0.001 to 1.04 (mean $=0.195, n=107$ ); with a mean of 0.26 and range of 0.002 to 1.04 in the surface $(n=83, \leq 200 \mathrm{~m})$, and mean of 0.009 and range of 0.001 to 0.056 at depth $(n=26,>200 \mathrm{~m})$. Other studies in the Ross Sea measured 2.08 $\pm 0.05 \mathrm{pmol} \mathrm{L} \mathrm{L}^{-1} \mathrm{~d}^{-1}$ uptake in the $>2 \mu \mathrm{m}$ size fraction, with the smaller $(0.2-2 \mu \mathrm{m})$ size fraction utilizing $0.88 \pm 0.27 \mathrm{pmol} \mathrm{L}^{-1} \mathrm{~d}^{-1}$ (Bertrand et al., 2007). An important caveat is the different radiotracer methods used in the Bertrand et al. (2007) study, where an additional $40 \mathrm{pM}$ of cold $\mathrm{B}_{12}$ was added, and likely overestimated in situ uptake rates compared to the values presented from this cruise. While this method may overestimate in situ uptake rates, it yields comparable measurements across sample locations and depths and is especially useful when $\mathrm{B}_{12}$ concentrations are unknown or not able to be measured. It is possible that using our approach introduced non-tracer level additions of $\mathrm{B}_{12}$ if ambient concentrations were well below pM levels. Each approach has its limitations, especially if $\mathrm{B}_{12}$ cannot be directly measured from field samples. Despite these limitations, the tracer-levels additions were chosen for this study to capture as close to real uptake rates for this trace organic.

\subsubsection{Bacterioplankton size fractionated cobalt and cobalamin uptake}

A proxy for bacterioplankton uptake was derived by calculating the difference between the measured ${ }^{57} \mathrm{Co}$ uptakes for the $\geq 0.2 \mu \mathrm{m}$ size fraction (total community) and the 
$\geq 3 \mu \mathrm{m}$ size fraction (phytoplankton community). The difference is an estimate of the contribution of bacterioplankton that are non-particle associated. The assumption made with this measurement is that there are negligible amounts of bacteria less than $3 \mu$ msize range in this environment. Prior studies observed that upwards of $30 \%$ of a diatom-dominated phytoplankton community's $\mathrm{B}_{12}$ assimilation was by the bacterial size fraction $(0.2-2 \mu \mathrm{m})$ (Bertrand et al., 2007). In McMurdo Sound sea ice, less than $3 \%$ of the diatom-dominated sea ice community could be attributed to the bacterial size fraction $(0.2-1 \mu \mathrm{m})$ (Taylor and Sullivan, 2008). The range of bacterioplankton $\mathrm{B}_{12}$ uptake rates remains to be constrained through controlled laboratory studies.

In this study, bacterioplankton ${ }^{57} \mathrm{Co}$ uptake had a mean of $1.48 \%$ and ranged from $0.0 \%$ to $19.42 \%(n=70)$. Bacterioplankton Co uptake rates were on average $0.15 \mathrm{pmol} \mathrm{L} \mathrm{L}^{-1} \mathrm{~d}^{-1}$ and ranged from $0.0 \mathrm{pmol} \mathrm{L}{ }^{-1} \mathrm{~d}^{-1}$ to $1.27 \mathrm{pmol} \mathrm{L}^{-1} \mathrm{~d}^{-1}(n=50)$. The bacterioplankton proxy also indicates a substantial contribution to $\%{ }^{57} \mathrm{~B}_{12}$ uptake, with a mean of $6.51 \%$ and range from $0.0 \%$ to $39.67 \%$ ( $n=103)$. Lastly, the bacterioplankton fractional contribution to uptake ranged from $2.6 \%$ to $74 \%$ $($ mean $=34 \%, n=40)$ for $\mathrm{Co}$, and from $4 \%$ to $85 \%($ mean $=42 \%, n=40)$ for $\mathrm{B}_{12}$ (across all stations).

An example of the change in fractional contribution to uptake by the phytoplankton size fraction and bacterioplankton size fraction is in Figure 3-9. There is a higher contribution of the smaller-size fraction (bacterioplankton proxy) to $\mathrm{B}_{12}$ uptake at depth; and a larger contribution of the larger phytoplankton size fraction to uptake in the surface euphotic zone, corresponding to fluorescence. A few samples of Co uptake at depth confirm a small uptake rate of $0.036 \mathrm{pmol} \mathrm{L}^{-1} \mathrm{~d}^{-1}(n=9)$, likely a result of lower biomass, slower growth in deep waters, and sufficient dCodue to minimal scavenging. According to genomic sequence analysis, many Antarctic marine bacterial strains are also auxotrophic for $\mathrm{B}_{12}$, requiring an exogenous source of the trace organic vitamin (Bertrand et al., 2011a; Romine et al., 2017). Bacterial ribonucleotide reductase (RNR) enzymes, use either $\mathrm{Fe}$ or $\mathrm{B}_{12}$. Some bacteria have been observed to only transcribe the gene encoding Fe-dependent RNR under low cell quotas of $\mathrm{B}_{12}$ (Borovok et al., 2006).

\subsubsection{Summary of uptake study results}

Across all stations and for all regions, $\%{ }^{57} \mathrm{Co}$ and ${ }^{57} \mathrm{~B}_{12}$ uptake are significantly correlated $(\mathrm{p} \leq 0.001)$ for both the $\geq 0.2 \mu \mathrm{m}$ and $\geq 3 \mu \mathrm{m}$ size fractions, suggesting a strong coupling of $\mathrm{Co}$ and $\mathrm{B}_{12}$ cycling overall (regression not included, $R^{2}$ ranges from 0.38 to 0.87 ). In some sites $\%{ }^{57} \mathrm{Co}$ and ${ }^{57} \mathrm{~B}_{12}$ uptake appear more decoupled; for example Station 20 with minimal chlorophyll and a dinoflagellate community, where there was $\mathrm{B}_{12}$ uptake and nearly no Co uptake (Fig.3-2). The observed percent ${ }^{57} \mathrm{Co}$ and ${ }^{57} \mathrm{~B}_{12}$ uptake rates are highest in the surface photic zone, consistent with its removal as a micronutrient via phytoplankton and bacterioplankton uptake. The size-fractionated $\%{ }^{57} \mathrm{Co}$ and $\%{ }^{57} \mathrm{~B}_{12}$ uptake results confirm that phytoplankton are the largest term in the $\mathrm{Co}$ and $\mathrm{B}_{12}$ uptake flux, but that in certain regions bacterioplankton

also contribute a substantial amount of uptake. Finally, both $\%{ }^{57} \mathrm{Co}$ and ${ }^{57} \mathrm{~B}_{12}$ uptake have strong and significant correlations with salinity, with higher rates in fresher, 
Figure 3-9: An example of the spatially distinct contribution of phytoplankton vs. bacterioplankton to the total community ${ }^{57} \mathrm{~B}_{12}$ uptake. In Station 15, bacteria and archaea were more important for removal of $\mathrm{dB}_{12}$ relative to phytoplankton below the fluorescence maximum and further at depth. Total Co is plotted alongside to show the surface draw down and assimilation into biomass.

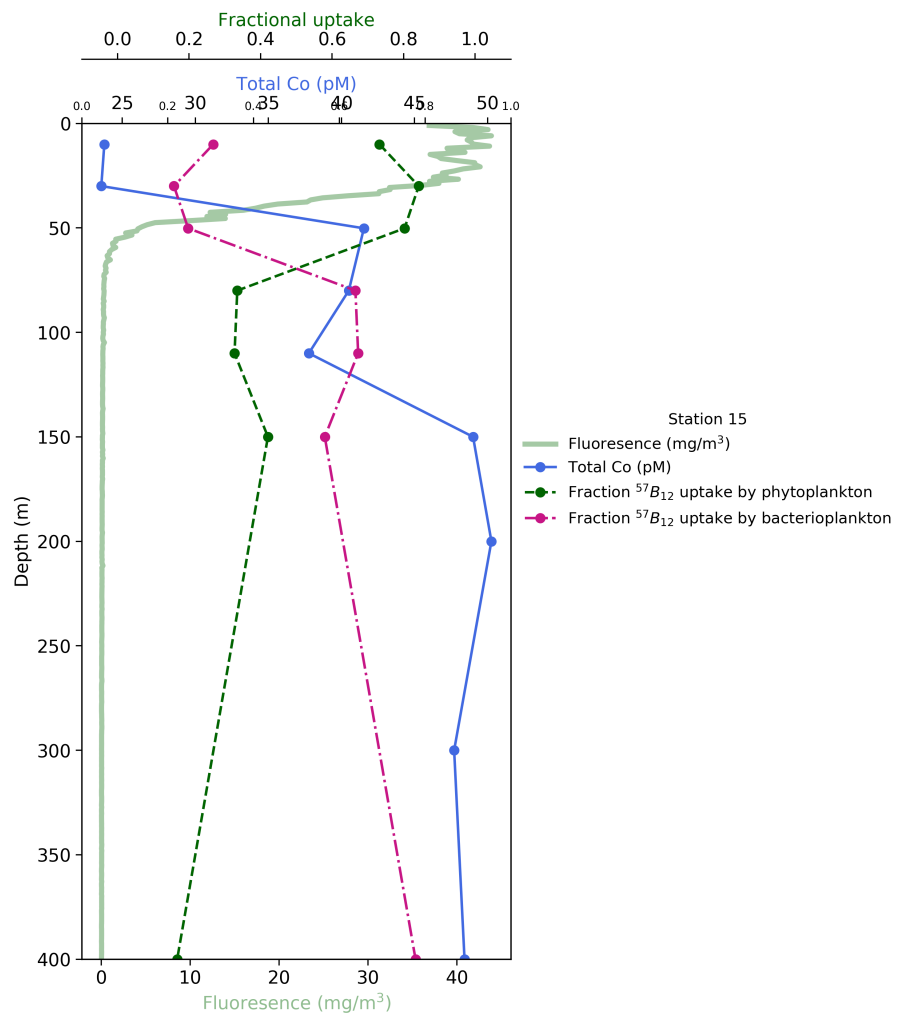

warmer surface waters (mostly AASW Summer, AASW and mCDW water masses) that are likely to have increased input of meltwater Fe that can stimulate growth and nutrient draw down (Fig.3-10). These results indicate a rapid ecological cycling and high cobalamin flux into microbial biomass in Antarctic waters, contributing to the observed low concentration (standing stock) of vitamin $\mathrm{B}_{12}$ and potential for vitamin limitation. Furthermore, given that bacterial cobalt co-oxidation with manganese oxides is a minor contribution to Co removal in this region, these uptake results indicate that cobalt is cycled primarily in the surface mixed layer by the euphotic-zone marine microbial community.

\subsubsection{Community-driven patterns of cobalt uptake}

In this section, the patterns of ${ }^{57} \mathrm{Co}$ and ${ }^{57} \mathrm{~B}_{12}$ uptake are compared to phytoplankton chemotaxonomic pigment distributions to detect correlations between the resident microbial community and micronutrient assimilation. The analysis is focused on trace metal samples from the Amundsen-Ross Transect and a compilation of all sample data, subset by region (Amundsen Sea, Ross Sea, and Terra Nova Bay). The transect is chosen for its geographic coverage over the span of one week (January 3, 2018 January 9, 2018), whereas the regional compilation is over forty days (January 3, 2018 - February 18, 2018). As expected in this area of seasonal extremes, the regional compilation covers a range of ecological dynamics over time. The Pearson's correlation was run to assess the existence and strength of a linear relationship between $\%{ }^{57} \mathrm{Co}$ uptakes, $\mathrm{Co}_{\text {total }}$, and phytoplankton pigment concentrations. Due to the relatively 


\begin{tabular}{|c|c|c|c|c|}
\hline Size Fractions & $\begin{array}{l}{ }_{\%}^{57} \mathrm{Co} \% \text { Uptake } \\
\%\end{array}$ & $\begin{array}{l}\text { Co Uptake } \\
\mathrm{x} \mathrm{Co}_{\text {total }} \mathrm{pMd}^{-1}\end{array}$ & $\begin{array}{l}{ }_{\%}^{57} B_{12} \% \text { Uptake } \\
\%\end{array}$ & $\begin{array}{l}\text { Est. } \mathbf{B}_{12} \text { Uptake } \\
\times 1 \mathrm{pMd}^{-1}\end{array}$ \\
\hline \multicolumn{5}{|c|}{ Total Community } \\
\hline Mean (n) & $4.42(76)$ & $0.61(53)$ & $17.77(107)$ & 0.195 \\
\hline Range & $(0.04-26.46)$ & $(0.01-3.06)$ & $(0.1-94.16)$ & $(0.001-1.04)$ \\
\hline \multicolumn{5}{|l|}{ Phytoplankton } \\
\hline Mean (n) & $3.26(76)$ & $0.5(55)$ & $12.84(108)$ & 0.14 \\
\hline Range & $(0.03-18.77)$ & $(0.007-2.87)$ & $(0.06-75.89)$ & $(0.0007-0.83)$ \\
\hline \multicolumn{5}{|c|}{ Bacterioplankton } \\
\hline Mean (n) & $1.48(70)$ & $0.15(50)$ & $6.51(103)$ & 0.07 \\
\hline Range & $(0.0-19.42)$ & $(0.0-1.27)$ & $(0.0-39.67)$ & $(0.0-0.44)$ \\
\hline
\end{tabular}

Table 3.2: Summary of cobalt and cobalamin uptake (as a percent of radiotracer taken up), estimated uptake rates ( $\mathrm{pmol} \mathrm{L}{ }^{-1}$ day $^{-1}$ ) based on total Co measurements, and estimated ambient $\mathrm{B}_{12}$ concentration of $1 \mathrm{pM}$. The total community uptake is based on $\geq 0.2 \mu \mathrm{m}$ filter samples. Phytoplankton community uptake is assimilation collected on $\geq 3 \mu \mathrm{m}$ filters, capturing most of the larger phytoplankton in Antarctic waters. The bacterioplankton uptake is a proxy calculated by subtracting the phytoplankton assimilation from the total community size-fraction uptake.

small sample size, the non-parametric Spearman rank-order correlation $\left(r_{s}\right)$ was also used to investigate any underlying monotonic relationships between variables. In these coastal Antarctic waters, the chemotaxonomic indicator pigments 19-Hex and Fuco reflect $P$. antarctica and diatom species, respectively.

\section{Comparing stations on the Amundsen Sea to Ross Sea Transect}

A closer examination of the stations along the transect from the Amundsen Sea to Terra Nova Bay enables a comparison between diatom- and P. antarctica-dominated regions, as compiled in Fig.3-2 and Fig.3-12 that contain both hydrographic and biochemical profiles. In all stations with phytoplankton blooms, total dCo was minimal at the surface, following a typical nutrient-like profile, and corresponding to $\%{ }^{57} \mathrm{Co}$ and ${ }^{57} \mathrm{~B}_{12}$ uptake measurements. A notable exception is Station 20 that had lower fluorescence, chlorophyll a, and ${ }^{57} \mathrm{~B}_{12}$ and ${ }^{57} \mathrm{Co}$ uptake that appear decoupled. The Amundsen Sea stations were dominated by massive blooms of $P$. antarctica that influence both $\%{ }^{57} \mathrm{Co}$ and ${ }^{57} \mathrm{~B}_{12}$ uptake, while the Ross Sea sites had mixed or diatom-dominated communities. Next is a closer examination of the stations along the Amundsen-Ross Transect (TM Stations 10, 15, 20, and 22).

Station 11: This site was along the Amundsen Sea continental shelf and had a mixed community of predominantly P. antarctica $(>70 \%$ of $\mathrm{Chl}$ a attributed to high iron haptophytes) and a minor diatom community $(<10 \% \mathrm{Chl}$ a attributed to diatoms), according to ChemTax analysis (personal communication with G. DiTullio and N. Schanke). There was a relatively deep mixed layer of $37 \mathrm{~m}$ with a cap of warmer, fresher AASW Summer water in the surface. In the surface $(10 \mathrm{~m})$ vs. deep $(50 \mathrm{~m})$ waters, phytoplankton pigments were highest in the surface (19-Hex ranged from $1729.6 \mathrm{ng} \mathrm{L}^{-1}$ to $77.1 \mathrm{ng} \mathrm{L}^{-1}$ and Fuco from $268.6 \mathrm{ng} \mathrm{L}^{-1}$ to $41.4 \mathrm{ng} \mathrm{L}^{-1}$ ); and 

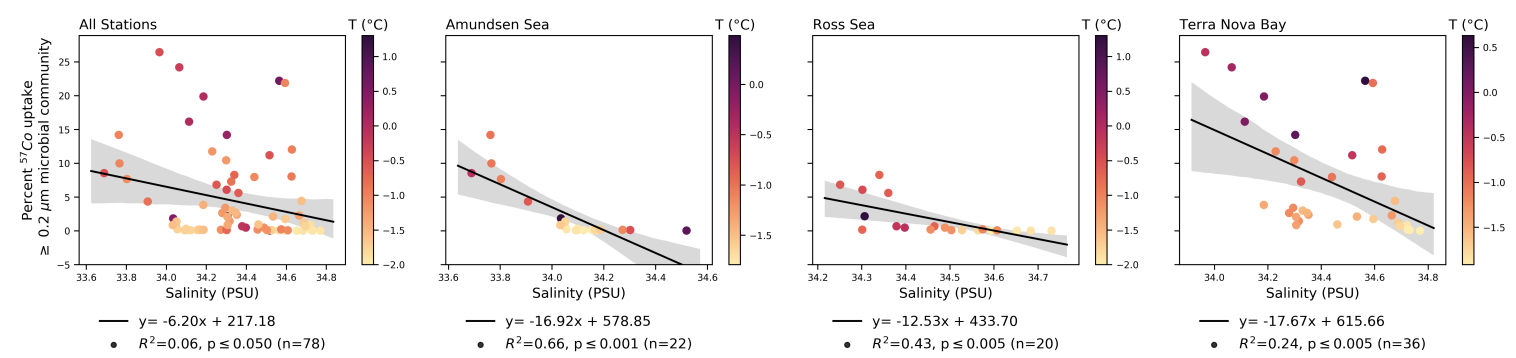

- $R^{2}=0.06, p \leq 0.050(n=78)$

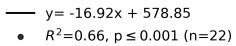

- $y=-12.53 x+433.70$

- $y=-17.67 x+615.66$

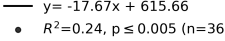
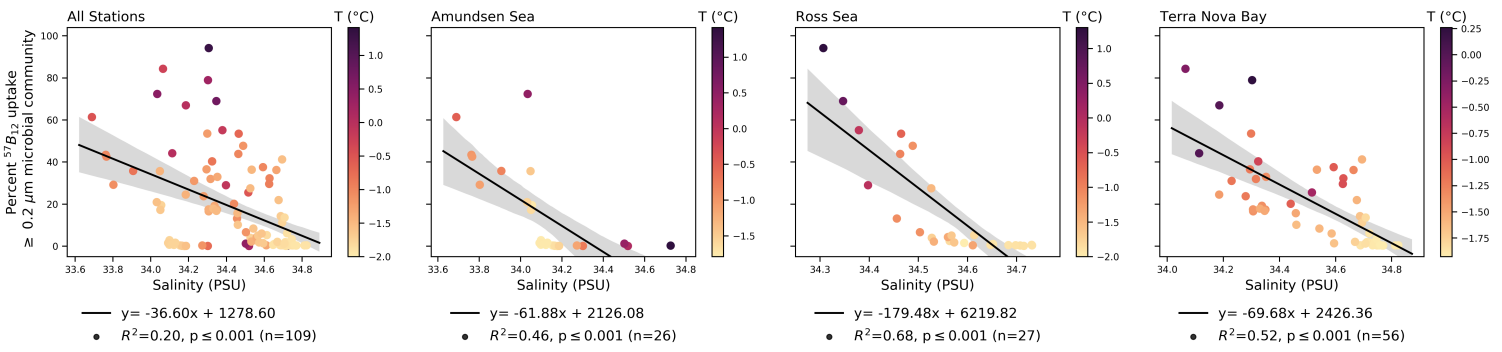

Figure 3-10: Salinity has a strong and significant correlation with $\%$ uptake across all regions. More $\%{ }^{57} \mathrm{Co}$ and ${ }^{57} \mathrm{~B}_{12}$ uptake happens in fresher surface waters (AASW Summer, AASW, and $\mathrm{mSW}$ water masses) where there is likely more meltwater iron input.

biogenic silica follow suit from $6.11 \mu \mathrm{M}$ to $0.59 \mu \mathrm{M}$. Notably, there was only a minor surface reduction in dissolved silicate from $80.5 \mu \mathrm{M}$ to $75.9 \mu \mathrm{M}$ suggesting that the Fuco present was likely in P. antarctica. At this site, $\mathrm{Co}_{\text {total }}$ is reduced from $41.2 \mathrm{pM}$ at $50 \mathrm{~m}$ to $21.4 \mathrm{pM}$ at $10 \mathrm{~m}$. Percent ${ }^{57} \mathrm{~B}_{12}$ uptake extends to $75 \mathrm{~m}$ on the $\geq 3 \mu \mathrm{m}$ filter fractions, past where phytoplankton pigments are present (ending at around $50 \mathrm{~m}$ ), and may reflect particle-associated ${ }^{57} \mathrm{~B}_{12}$ uptake by bacterioplankton on sinking $P$. antarctica colonies.

Station 15: At this site, a massive and productive bloom of colonial $P$. antarctica dominated the marine microbial community (ChemTax analysis indicates that $>80 \%$ of the Chl a can be attributed to $P$. antarctica expressive of high iron conditions, personal communication with G. DiTullio). The 19-Hex concentrations at this station approached $4020.9 \mathrm{ng} \mathrm{\textrm {L } ^ { - 1 }}$ and fluorescence reached a maximum of $43.9 \mathrm{mg} \mathrm{m}^{-3}$. The comparatively high rates of $\%$ uptake suggest that the $P$. antarctica bloom and associated microbiome are drawing down $\mathrm{Co}_{\text {total }}$ in the upper water column, where dCo drops from $42 \mathrm{pM}$ a depth to $<25 \mathrm{pM}$ in the surface where chlorophyll a and fluorescence are highest. Similar to Station 11, the fluorescence signal also extends to the surface, reflecting both an undefined mixed layer and the buoyant nature of $P$. antarctica colonies, corroborating shipboard observations of floating, "frothed" masses of colonies and mucus at this station. ${ }^{57} \mathrm{~B}_{12}$ uptake extends to $75 \mathrm{~m}$ on the $\geq 3 \mu \mathrm{m}$ filter fractions, past where phytoplankton pigments are present (ending at around $50 \mathrm{~m}$ ), and may reflect particle-associated ${ }^{57} \mathrm{~B}_{12}$ uptake by bacterioplankton on sinking $P$. antarctica colonies. 
Station 20: Station 20 had minimal fluorescence $\left(2.5 \mathrm{mg} \mathrm{m}^{-3}\right)$ and minimal \% uptake, though notably higher ${ }^{57} \mathrm{~B}_{12}$ uptake relative to ${ }^{57} \mathrm{Co}$ uptake. Compared to other stations, this site did not have a defined mixed layer and it had low fluorescence extending to $150 \mathrm{~m}$. There was a mixed community, according to pigment analysis, that included Fuco, 19-Hex, and Peridinin, the latter pigment is indicative of dinoflagellates. Total dCo is relatively uniform with depth with a concentration around $35 \mathrm{pM}$, with the dip at $50 \mathrm{~m}$, corresponding to a peak in fluorescence and $\%{ }^{57} \mathrm{~B}_{12}$ uptake, where it is reduced to $26 \mathrm{pM}$. At this station, $\%{ }^{57} \mathrm{~B}_{12}$ uptake and $\%^{57} \mathrm{Co}$ uptake appear decoupled, with the resident microbial community having a higher demand for $\mathrm{B}_{12}$ relative to Co. This preference for $\mathrm{B}_{12}$ over $\mathrm{Co}$ is in line with studies observing that many dinoflagellates are $\mathrm{B}_{12}$-auxotrophs (Croft et al., 2006).

Station 22: Station 22 was in Terra Nova Bay and had a diatom bloom, as indicated by high Fucoxanthin concentrations $\left(287.8 \mathrm{ng} \mathrm{L}^{-1}\right.$ to $\left.31.7 \mathrm{ng} \mathrm{L}^{-1}\right)$ combined with opposing surface $(10 \mathrm{~m})$ vs. deep $(75 \mathrm{~m})$ biogenic silica $(3.57 \mu \mathrm{M}$ to $1.20 \mu \mathrm{M})$ and dissolved silicate $(27.3 \mu \mathrm{M}$ to $74.3 \mu \mathrm{M})$, Fig.3-11. Interestingly, the phytoplankton community $\%{ }^{57} \mathrm{Co}$ and $\mathrm{B}_{12}$ uptake rates follows a similar spatial pattern with depth, compared to Stations 11, 15, and 20 that appear to have more decoupled uptake patterns in the euphotic zone. There was a distinct and shallow mixed layer that extended to $23 \mathrm{~m}$. Total dCo is reduced to $11.8 \mathrm{pM}$ at $10 \mathrm{~m}$, from $30.5 \mathrm{pM}$ at $75 \mathrm{~m}$, with ${ }^{57} \mathrm{Co}$ uptake rates reaching a maximum of $18.76 \%$ at $10 \mathrm{~m}$, and a high corresponding Co uptake rate of $2.23 \mathrm{pmol} \mathrm{L}^{-1} \mathrm{~d}^{-1}$. At this diatom-dominated station, ${ }^{57} \mathrm{Co}$ and ${ }^{57} \mathrm{~B}_{12}$ uptake appear spatially coupled, with uptake extending to $100 \mathrm{~m}$ depth. The $\%$ ${ }^{57} \mathrm{~B}_{12}$ uptake rates ranged from 24.9 to 5.9 for the $>3 \mu \mathrm{m}$ size fraction in the upper $75 \mathrm{~m}$.

When considering all data, there is a relatively weak but significant correlation between Fuco and total community size-fraction $(\geq 0.2 \mu \mathrm{m}) \%{ }^{57} \mathrm{Co}$ and ${ }^{57} \mathrm{~B}_{12}$ uptake (Fig.3-13), $r^{2}=0.18(n=37)$ and $\left.r^{2}=0.28,(n=43)\right)$, but there was no significant correlation between 19-Hex and either $\%{ }^{57} \mathrm{Co}$ or ${ }^{57} \mathrm{~B}_{12}$ uptake. As the Hex:Fuco ratio suggests, the colonial bloom had different uptake behavior compared to regions of lower 19-Hex concentration. However, along the Amundsen-Ross Transect, there was a significant correlation between the phytoplankton size-fraction $(\geq 3 \mu \mathrm{m}){ }^{57} \mathrm{Co}$ uptake and diatom and P. antarctica pigments, Fig.3-14. Notably, the relationship between

Fuco and $\geq 3 \mu \mathrm{m}{ }^{57} \mathrm{Co}$ uptake was much stronger $\left(r^{2}=0.84, p \leq 0.001, n=15\right)$ than with 19-Hex $\left(r^{2}=0.28, p \leq 0.05, n=15\right)$, and even total chlorophyll a $\left(r^{2}=0.44\right.$, $n=15$, not shown). If only the phytoplankton size fraction is considered, then the correlation between Fuco and $\geq 3 \mu \mathrm{m}{ }^{57} \mathrm{~B}_{12}$ potential uptake is similar to 19 -Hex $\left(r^{2}=0.59, r^{2}=0.55, n=14\right.$, respectively), though less than to total chlorophyll a $\left(r^{2}=0.64, n=14\right.$, not shown).

\section{Regional correlations of phytoplankton pigments with Co and uptake}

A regional analysis reveals a strong, significant correlation between $\mathrm{Co}_{\text {total }}$ and Co uptake and diatoms, and not $P$. antarctica; with the interesting exception of $P$. antarctica being significantly correlated with uptake in the Amundsen Sea, where 
Figure 3-11: Station 22 in Terra Nova Bay had a diatom bloom, as indicated by high fluorescence $\left(\mathrm{mg} \mathrm{m}^{-3}\right)$, levels of Fucoxanthin $\left(\mathrm{ng} \mathrm{L}^{-1}\right)$, and biogenic silica $(\mu \mathrm{M})$ with corresponding low surface dissolved silicate $(\mu \mathrm{M})$.

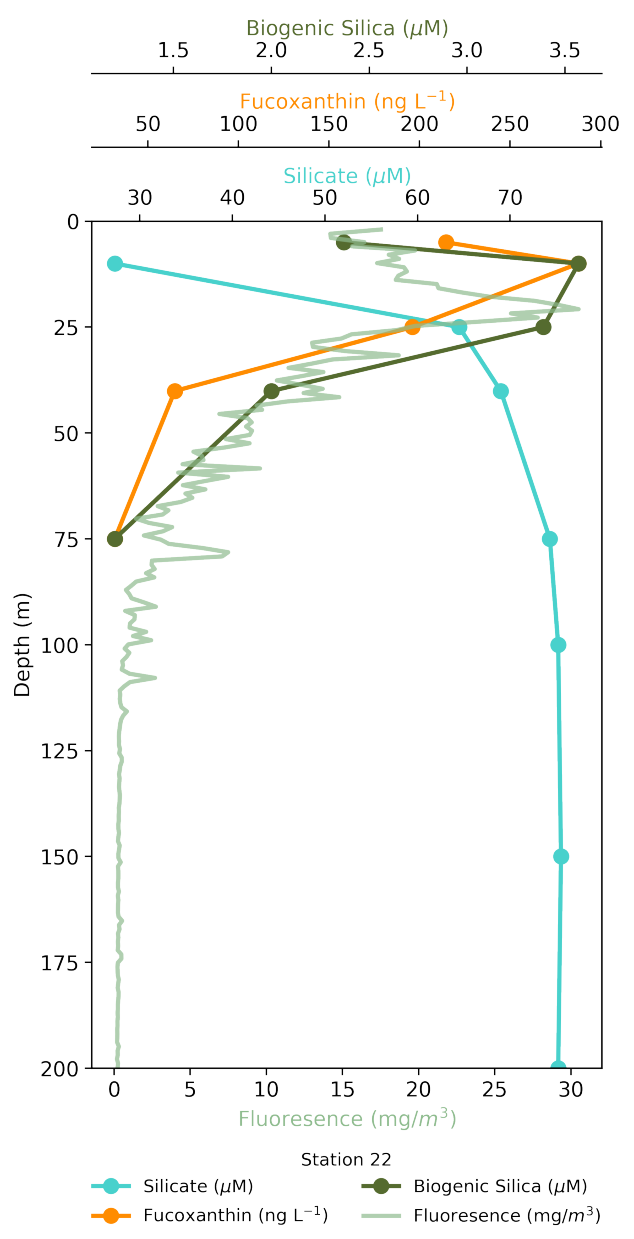



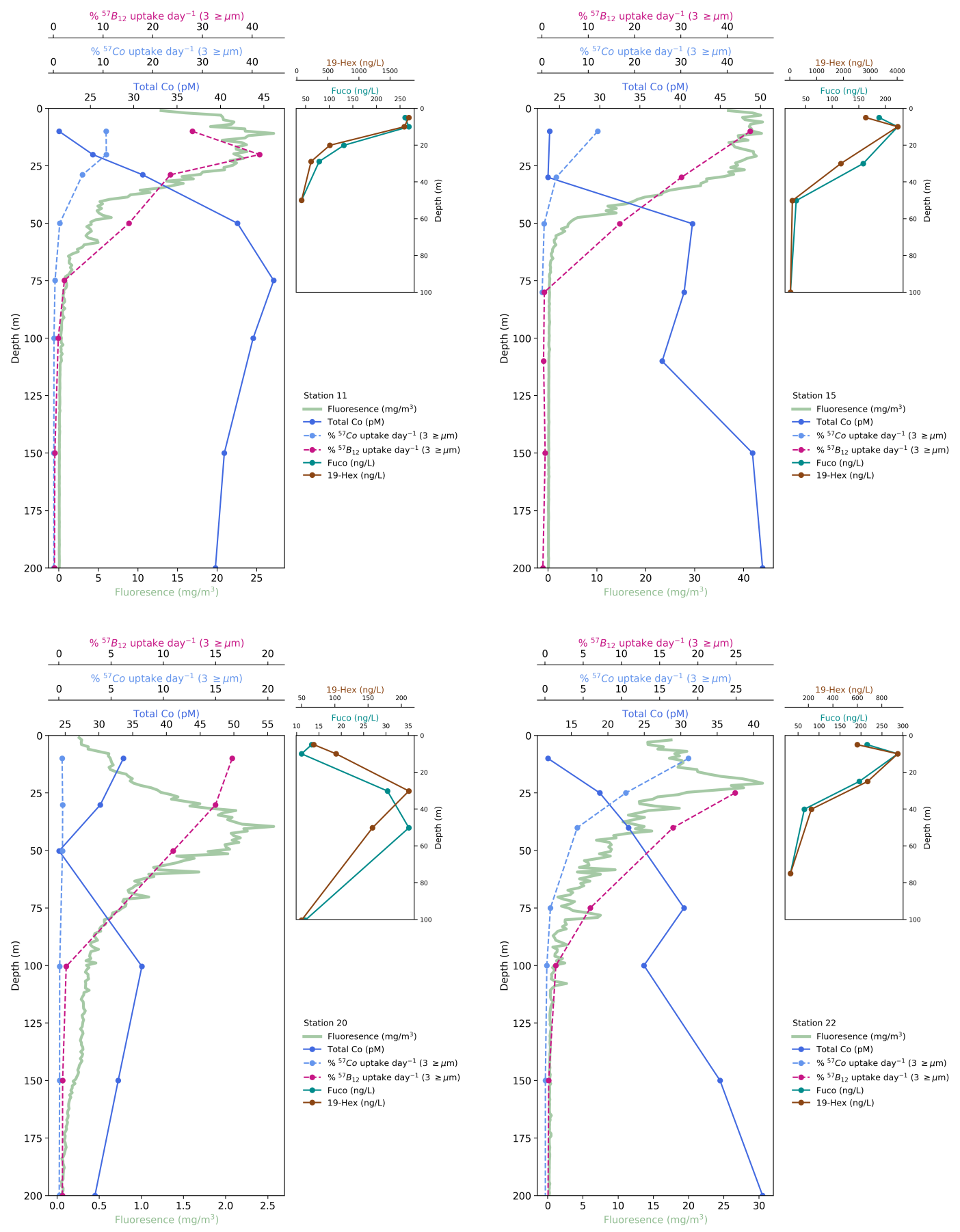

Figure 3-12: Profiles of fluorescence, $\%{ }^{57} \mathrm{Co}$ and ${ }^{57} \mathrm{~B}_{12}$ uptake $(\geq 3 \mu \mathrm{m}), \mathrm{Co}_{\text {total }}(\mathrm{pM})$, and chemotaxonomic phytoplankton pigments Fuco and 19-Hex $\left(\mathrm{ng} \mathrm{L}^{-1}\right)$. 

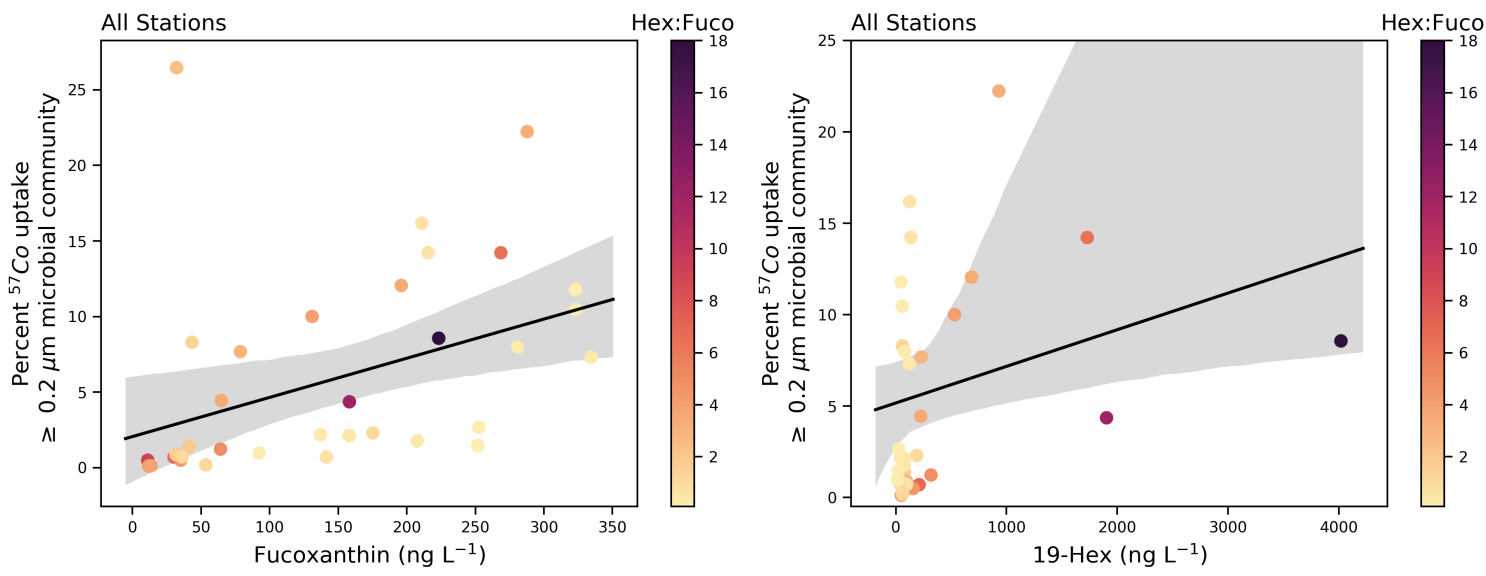

$y=0.03 x+2.06$

- $R^{2}=0.18, \mathrm{p} \leq 0.050(\mathrm{n}=37)$
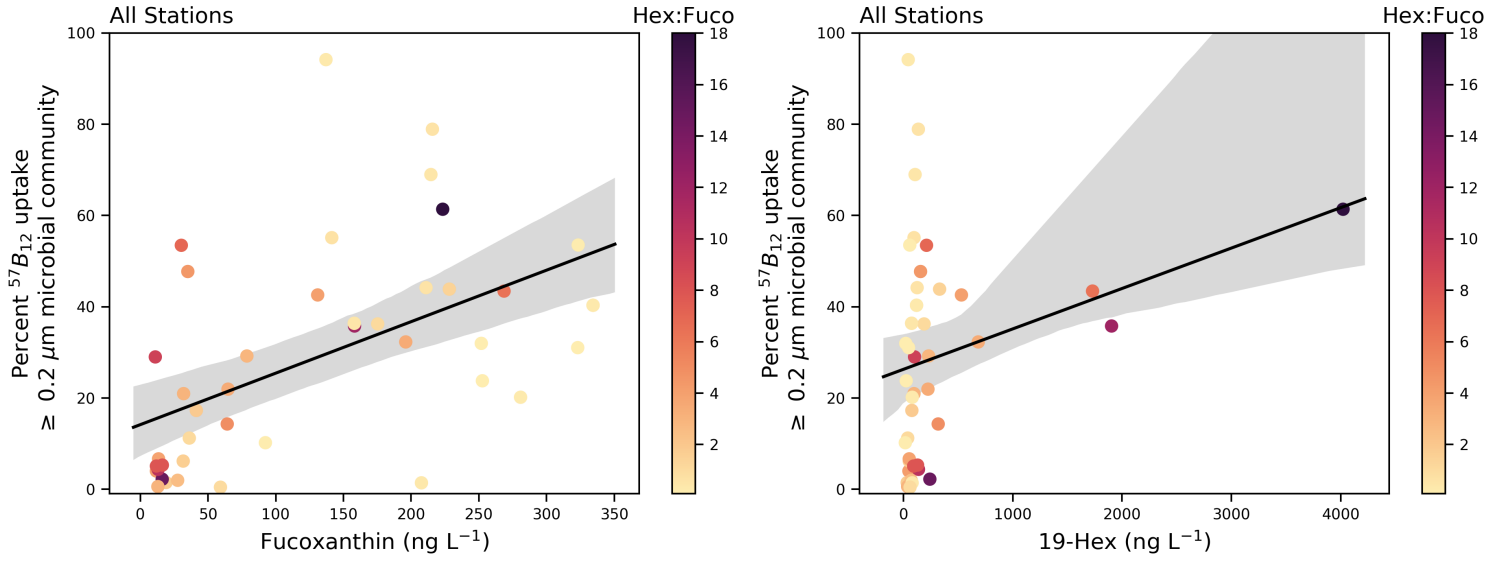

$-\mathrm{y}=0.11 \mathrm{x}+14.15$

$-y=0.01 x+26.28$

- $R^{2}=0.28, \mathrm{p} \leq 0.001(\mathrm{n}=43)$

- $R^{2}=0.07, \mathrm{p} \leq 0.082(\mathrm{n}=43)$

Figure 3-13: Potential Co and $\mathrm{B}_{12}$ uptake rates of the $\geq 0.2 \mu \mathrm{m}$ size fraction filter versus phytoplankton pigment concentration of diatom indicator pigment fucoxanthin (Fuco) (top and bottom left panels), and haptophyte $P$. antarctica pigment 19-hexanolyoxyfucoxanthin (19-Hex) (top and bottom right panels). Color overlay is the ratio of Hex:Fuco pigments to highlight where $P$. antarctica or diatoms were dominant. 

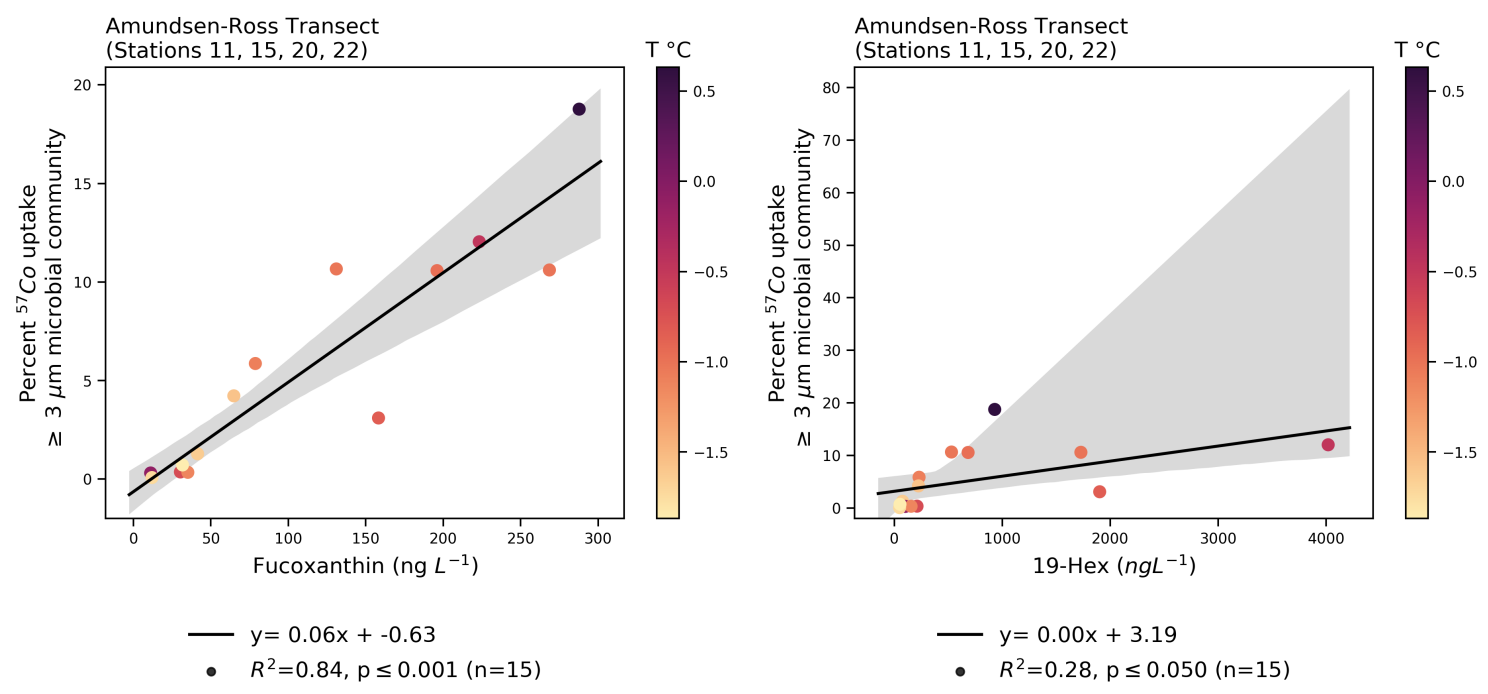

Figure 3-14: Over the Amundsen-Ross Transect, coexisting diatoms and P. antarctica displayed markedly different relationships to $\%{ }^{57}$ Co uptake. Diatom pigment Fuco had a very strong relationship to ${ }^{57}$ Co uptake, whereas $P$. antarctica pigment 19 -Hex had a much weaker correlation, though still significant.

there was a massive bloom in its colonial life stage, as seen in Figs.3-15 and 3-16. In the Amundsen sites, $\mathrm{Co}_{\text {labile }}$ was undetectable in the surface $(50 \mathrm{~m})$, and agrees with other observations from the Peru Upwelling region where type 4 haptophytes (Phaeocystis sp.) dominated where dCo was strongly complexed by organic ligands (Saito et al., 2004). When considering all stations, there is a significant and strong linear correlation between $\mathrm{Co}_{\text {total }}$ and fucoxanthin $\left(R^{2}=0.53, p \leq 0.001, n=43\right)$; this significant correlation holds for the Amundsen Sea, Ross Sea, and Terra Nova Bay (Fig.3-16). In contrast, there was no significant correlation found for the P. antarctica representative pigment $19-\mathrm{Hex}$ and $\mathrm{Co}_{\text {total }}$ in any region; however a strong, positive Spearman correlation was found only in the Amundsen region, which was statistically significant $\left(r_{s}=0.93, p \leq 0.001, n=9\right)$.

Cobalt uptake: Across all stations, there is a significant and moderate linear correlation between Co uptake and Fuco, and a weak but significant correlation with 19-Hex. Despite the expansive bloom of P. antarctica in the Amundsen-Ross Transect, 19-Hex is not significantly correlated to Co uptake. Rather, within this region, the diatom indicator pigment Fuco had a strong and significant positive correlation to Co uptake. These results (Fig.3-15), suggest that even within a predominantly $P$. antarctica-dominated region (Amundsen) and where these phytoplankton types coexist (Terra Nova Bay, Station 22), diatoms are driving the observed Co uptake.

${ }^{57} \mathbf{B}_{12}$ uptake: Only in the Amundsen Sea was P. antarctica 19-Hex strongly and significantly correlated to $\%{ }^{57} \mathrm{~B}_{12}$ uptake in the total community size fraction $\left(R^{2}=0.77, p \leq 0.05\right)$ and $\%{ }^{57}$ Co uptake $\left(R^{2}=0.68, p \leq 0.05\right)$ (Fig.3-17). However, this relationship is not present in the $\geq 3 \mu \mathrm{m}$ size fraction, suggesting that it may be 
$P$. antarctica-bloom associated bacterioplankton that are driving ${ }^{57} \mathrm{~B}_{12}$ uptake and not the colonial cells. It is clear that the microbial community in the colonial $P$. antarctica blooms in the Amundsen Sea has different uptake behavior than the regions of low 19-Hex concentrations, which are most likely in the flagellated single cell stage (in regions with $<1000 \mathrm{ng} \mathrm{\textrm {L } ^ { - 1 }}$ ). Lastly, biogenic silica was significantly correlated with $\%{ }^{57} \mathrm{~B}_{12}$ uptake in the phytoplankton size fraction samples across all regions, with stronger relationships in the Ross Sea and Terra Nova Bay regions (Fig.3-18).

\subsection{Discussion}

Taken together, these data support the argument that diatoms are driving Co and $\mathrm{B}_{12}$ uptake across Antarctica's coastal seas, but that $P$. antarctica colonies may exert their influence on $\mathrm{Co}$ and $\mathrm{B}_{12}$ cycling under bloom conditions. These results support prior findings that diatoms dominate cobalt uptake in the community relative to $P$. antarctica (Saito et al., 2010), and, moreover, that diatoms respond to $\mathrm{B}_{12}$ addition with rapid growth, triggering a community shift in the seawater samples over the longer time frame of the incubation experiment (Bertrand et al., 2007). Figure 3-18 compares the relationships of diatom proxies (fucoxanthin and biogenic silica) to phytoplankton $\%{ }^{57} \mathrm{~B}_{12}$ uptake, and demonstrates a strong relationship between biogenic silica and $\mathrm{B}_{12}$ uptake in all stations, but a lack of relationship with fucoxanthin to uptake in the Amundsen Sea area with predominantly P. antarctica colonies (that also contain fucoxanthin). Additionally, these results agree with findings from Chapter 2, that $P$. antarctica is not auxotrophic for $\mathrm{B}_{12}$, because it contains both MetE and MetH and maintains a similar growth rate across six orders of magnitude $\mathrm{B}_{12}$ concentration, changing its proteomic response to $\mathrm{MetH}$ only under high $\mathrm{B}_{12}$ conditions. It is important to note that not all diatoms are $\mathrm{B}_{12}$-auxotrophs and many contain both MetE and MetH proteins, and would also contribute to $\mathrm{B}_{12}$ uptake.

Throughout this analysis, the Amundsen Sea P. antarctica bloom stood out compared to the sites in the Ross Sea and Terra Nova Bay with lower 19-Hex concentrations, where $P$. antarctica is likely in small colonies or the flagellated, singlecell morphotype. There are key physiological differences between the morphotypes that can influence their competition dynamics with polar diatoms. For example, $P$. antarctica has a lower iron half saturation constant as a single cell $\left(K_{M}=0.005 \mathrm{nM}\right.$ to $0.043 \mathrm{nM}$, with respect to growth (Coale et al., 2003)) compared to the colonial stage $\left(K_{M}=0.45 \mathrm{nM}\right.$, under low irradiance of $\left.20 \mu \mathrm{E} \mathrm{m}^{-2} \mathrm{~s}^{-1}\right)$ (Sedwick et al., 2007). Notably, $P$. antarctica has a lower Co requirement than diatoms $\left(K_{M, \mathrm{Co}}=1.9 \times 10^{-13}\right.$ and $3.6 \times 10^{-12}$ respectively)(Saito and Goepfert, 2008). ${ }^{8}$ Considering the life cycle stages of $P$. antarctica, and their distinct ecophysiologies and niches, is essential to interpreting the field samples in Antarctic waters.

\footnotetext{
${ }^{8}$ N.B. This value is not for a polar diatom.
} 


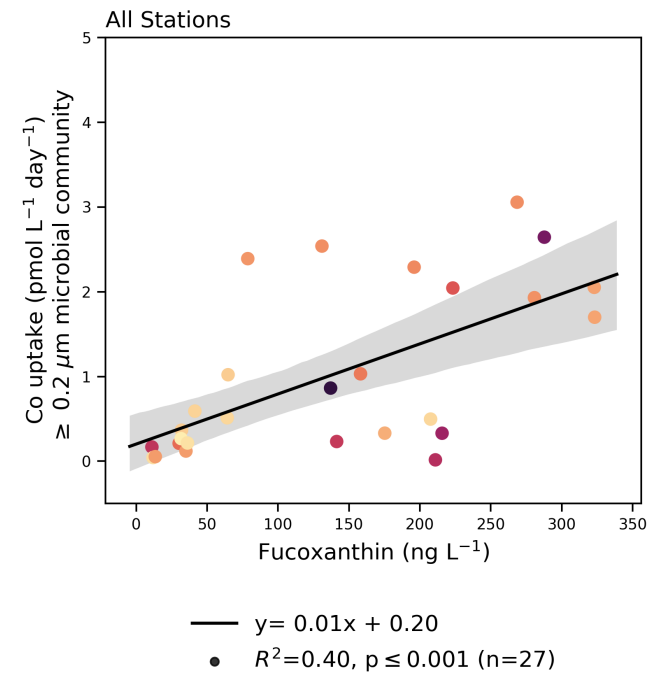

(a) Fuco, All Stations

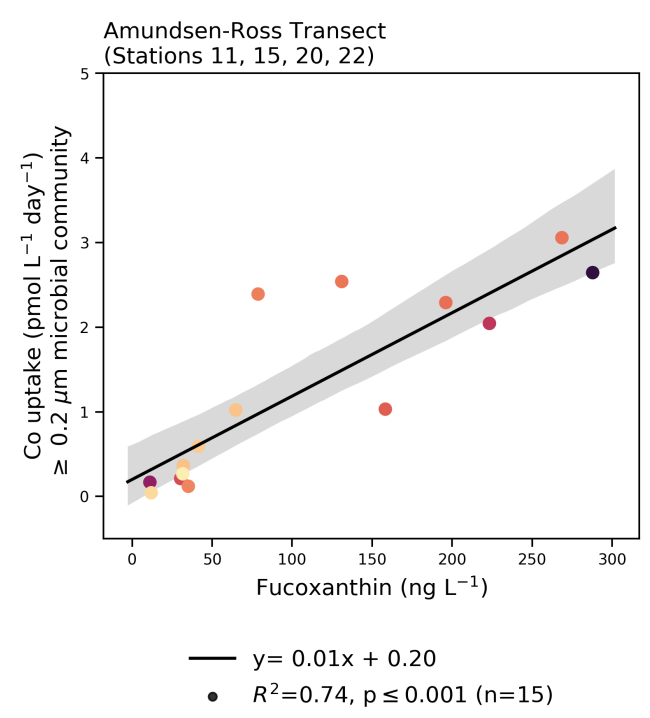

(c) Fuco, AR Transect
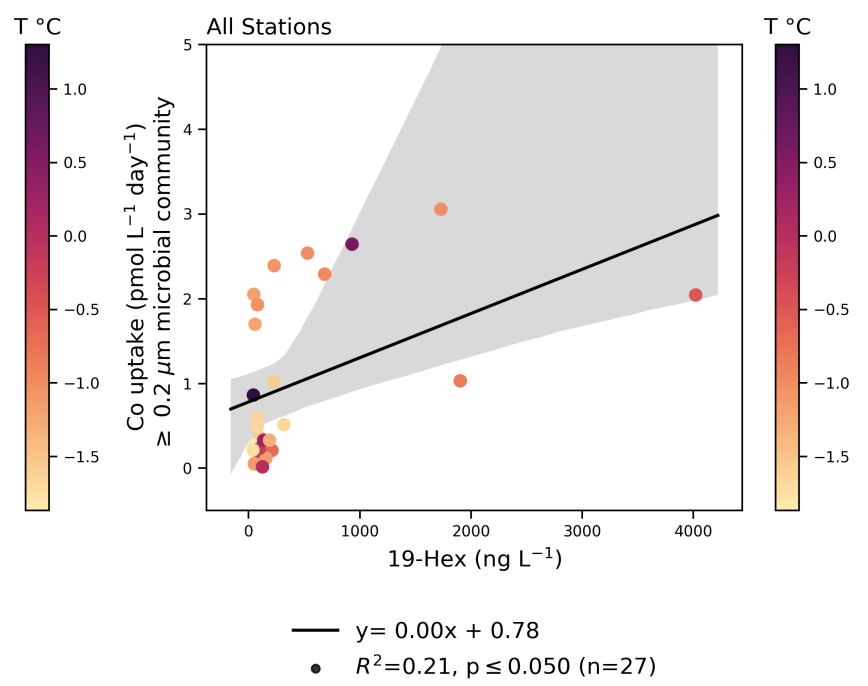

(b) 19-Hex, All Stations
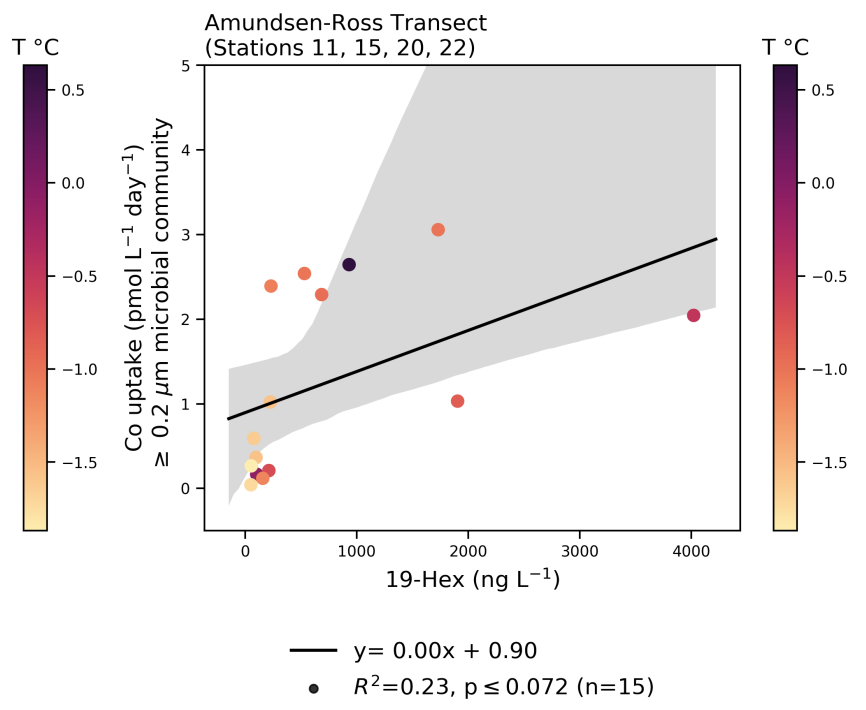

(d) 19-Hex, AR Transect

Figure 3-15: CICLOPS cobalt uptake rates $\mathrm{pmol} \mathrm{L}^{-1} \mathrm{~d}^{-1}$ versus phytoplankton pigment concentration of $(\mathbf{a}, \mathbf{c})$ diatom indicator pigment fucoxanthin (Fuco), and (b,d) haptophyte $P$. antarctica pigment 19-hexanolyoxyfucoxanthin (19-Hex). Cobalt uptake rates were significantly correlated to both pigments for all sample stations $(n=27)$ : Fuco $\left(r^{2}=0.40\right.$, $p \leq 0.001)$ and 19-Hex $\left(r^{2}=0.21, p \leq 0.05\right)$. In the Amundsen-Ross Transect (Stations $10,15,20,22, n=15)$, only a strong and significant correlation was found between cobalt uptake and Fuco $\left(r^{2}=0.74, p \leq 0.001\right)$ and not P. antarctica. 


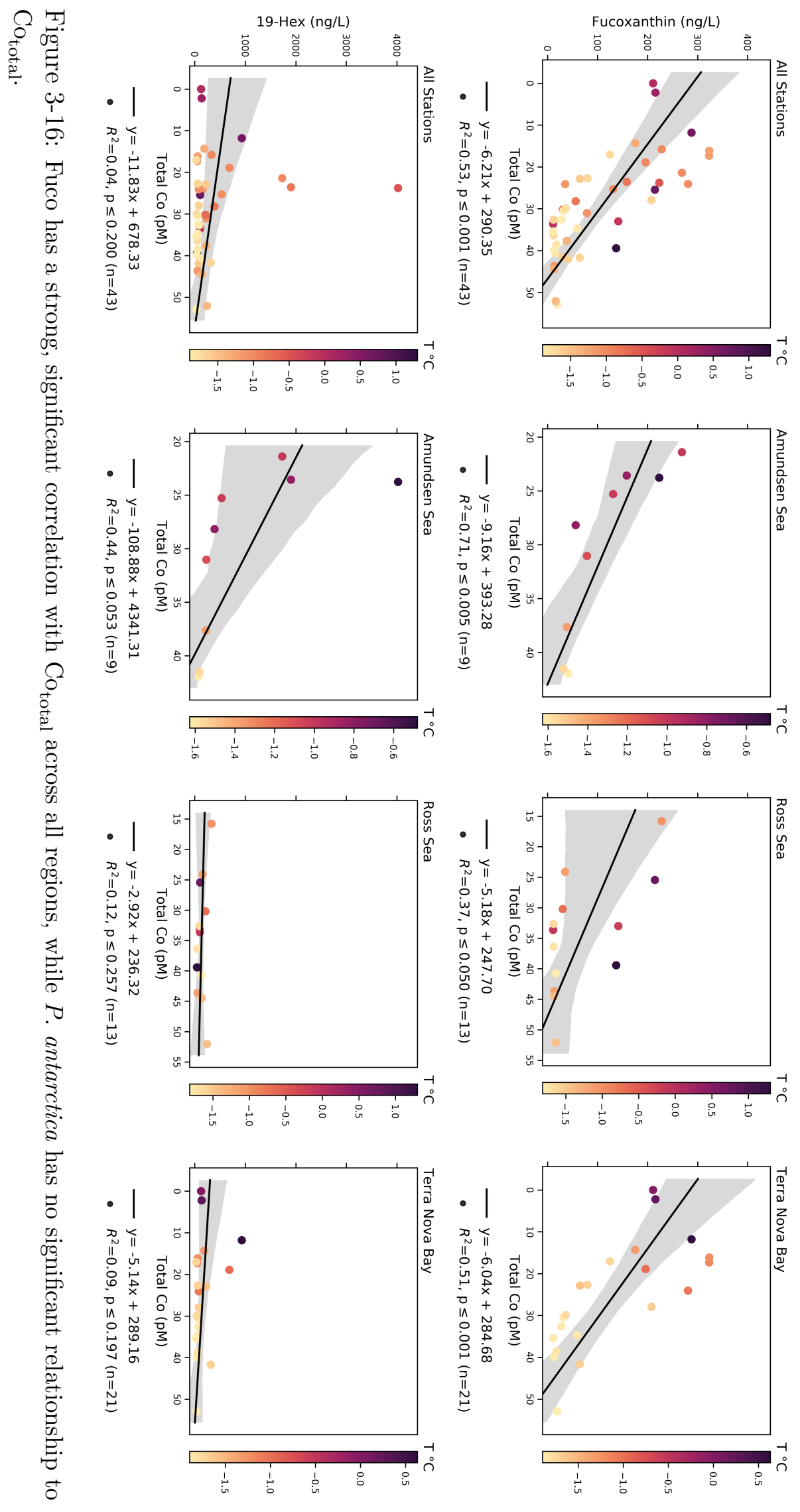



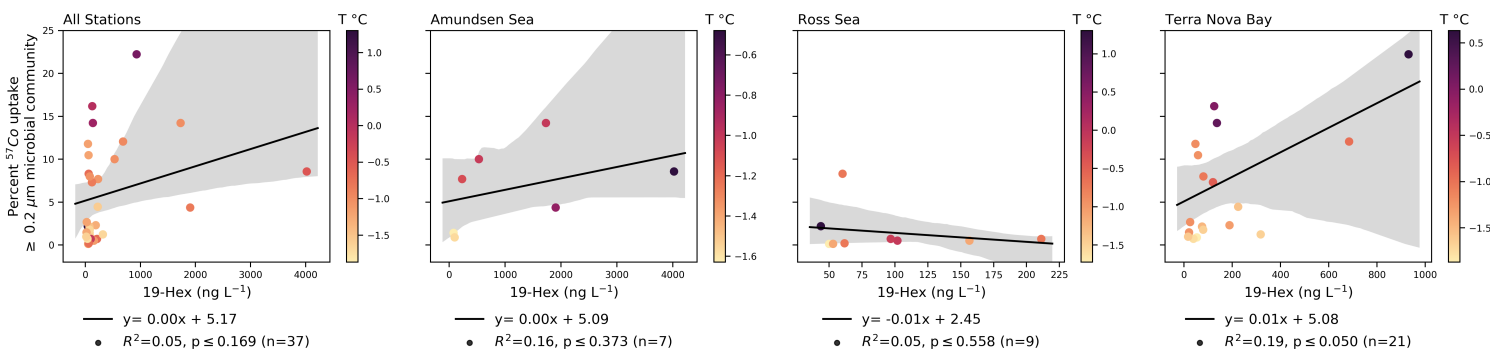

- $\begin{aligned} & y=0.00 x+5.09 \\ & R^{2}=0.16, p \leq 0.373(n=7)\end{aligned}$

- $y=-0.01 x+2.45$

- $R^{2}=0.19, p \leq 0.050(n=21)$
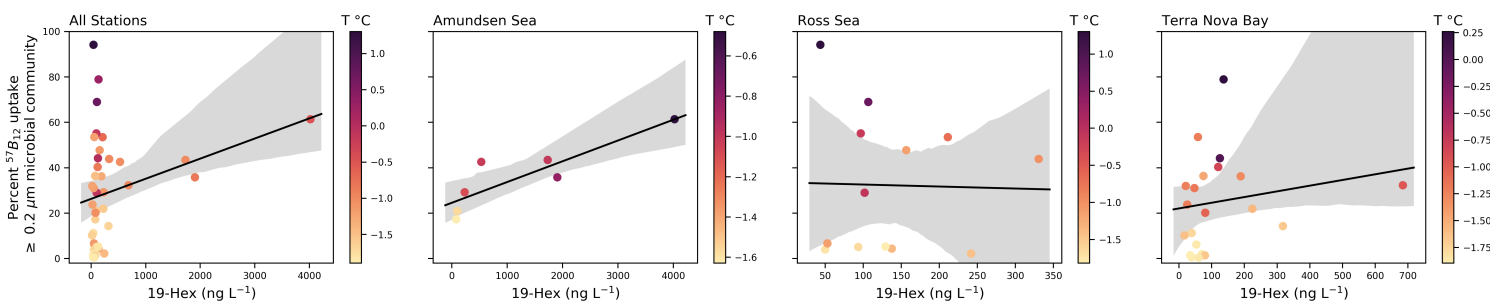

$y=0.01 x+26.28$
- $R^{2}=0.07, p \leq 0.082(n=43)$

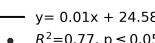

- $R^{2}=0.77, p \leq 0.050(n=7)$

- $y=-0.01 x+33.4$

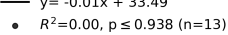
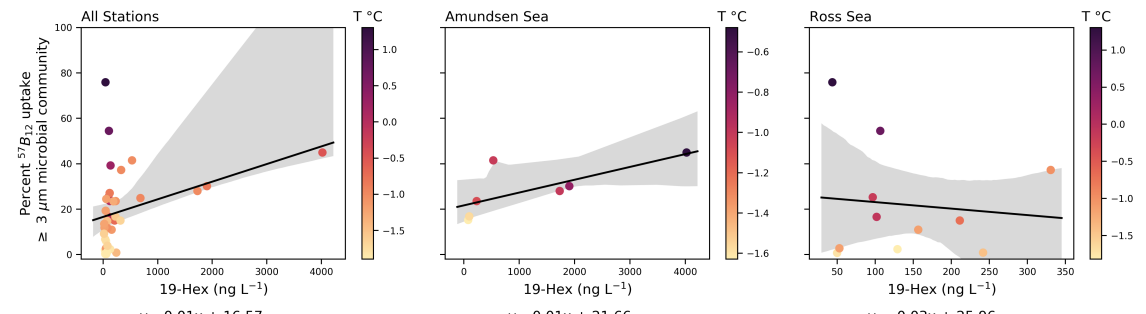

$y=0.03 x+22.07$

- $R^{2}=0.03, p \leq 0.414 \quad(n=23)$

Terra Nova Bay

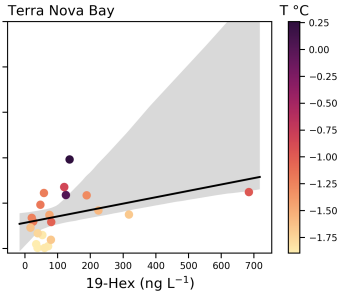

$y=0.01 x+21.66$
- $R^{2}=0.52, p \leq 0.069(n=7)$

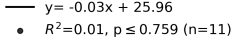

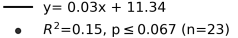

Figure 3-17: 19-Hex pigment has a strong, significant correlation with $\geq 0.2 \mu \mathrm{m} \%{ }^{57} \mathrm{~B}_{12}$ uptake only in the Amundsen Sea (and no relationship with ${ }^{57}$ Co uptake), the region dominated by colonial P. antarctica. 19-Hex was only weakly correlated with ${ }^{57}$ Co uptake in Terra Nova Bay and the relationship is driven by two sites with relatively high pigment concentrations. These patterns suggest that the blooming colonial life stage (associated with high 19-Hex levels) has different nutrient requirements than the single cell morphotype. Alternatively, the lack of relationship in the $\geq 3 \mu \mathrm{m}$ size fraction suggests that it may actually be $P$. antarctica-bloom associated bacterioplankton that are taking up ${ }^{57} \mathrm{~B}_{12}$ in the Amundsen Sea. 


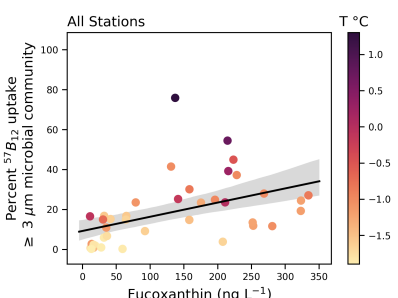

- $y=0.07 x+9.26$

- $R^{2}=0.22, p \leq 0.005(n=41$

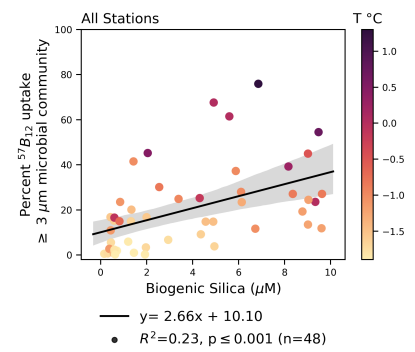

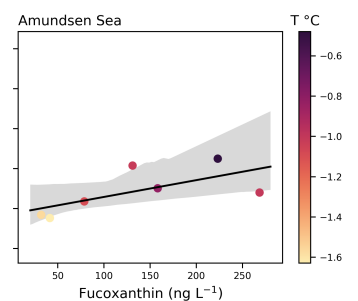

- $y=0.08 x+17.38$

- $R^{2}=0.44, \mathrm{p} \leq 0.103(\mathrm{n}=7)$

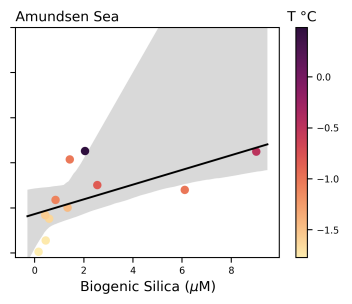

$\mathrm{y}=3.26 \mathrm{x}+17.29$
- $R^{2}=0.36, \mathrm{p} \leq 0.050(\mathrm{n}=11)$

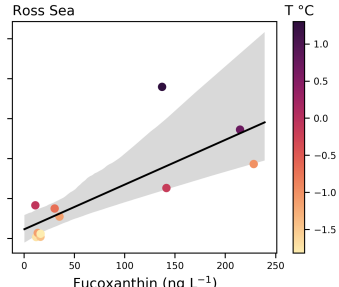

- $y=0.22 x+4.58$

- $R^{2}=0.61, p \leq 0.005$ ( $\left.n=11\right)$

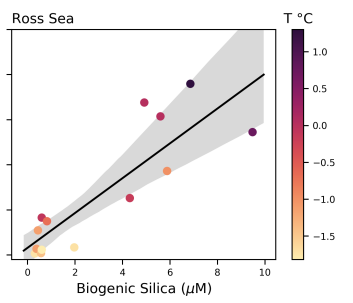

$-y=7.72 x+3.19$
- $R^{2}=0.74, p \leq 0.001(n=14)$

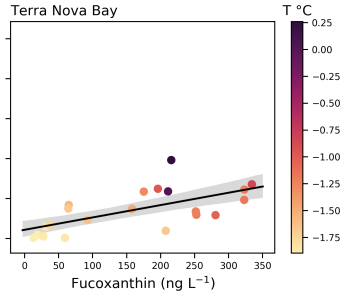

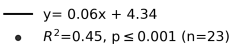

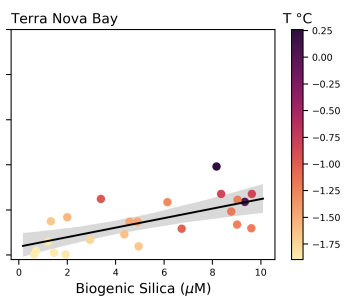

$y=2.11 x+3.71$
- $R^{2}=0.45, p \leq 0.001 \quad(n=23)$

Figure 3-18: Biogenic silica $\mu \mathrm{M}$ and $\%{ }^{57} \mathrm{~B}_{12}$ uptake in the phytoplankton size-fraction community $(\geq 3 \mu \mathrm{m})$ were significantly correlated throughout the water column the Amundsen Sea, Ross Sea, and in Terra Nova Bay. Whereas Fucoxanthin is significantly correlated with $\%{ }^{57} \mathrm{~B}_{12}$ uptake everywhere except the Amundsen Sea. These relationships support the notion of diatom-driven uptake (as indicated by biogenic silica), since $P$. antarctica also contains Fucoxanthin pigments and was not correlated to uptake where colonies were blooming. Linear regressions (black line), 95\% confidence interval (grey shaded area), p-values, and sample size (n) are shown for all sampled stations and the subset of three geographic regions. The Ross Sea sites had the strongest correlation between biogenic silica and ${ }^{57} \mathrm{~B}_{12}$ uptake. 


\section{A hypothesis for micronutrient-driven seasonal succession in Antarctic coastal waters}
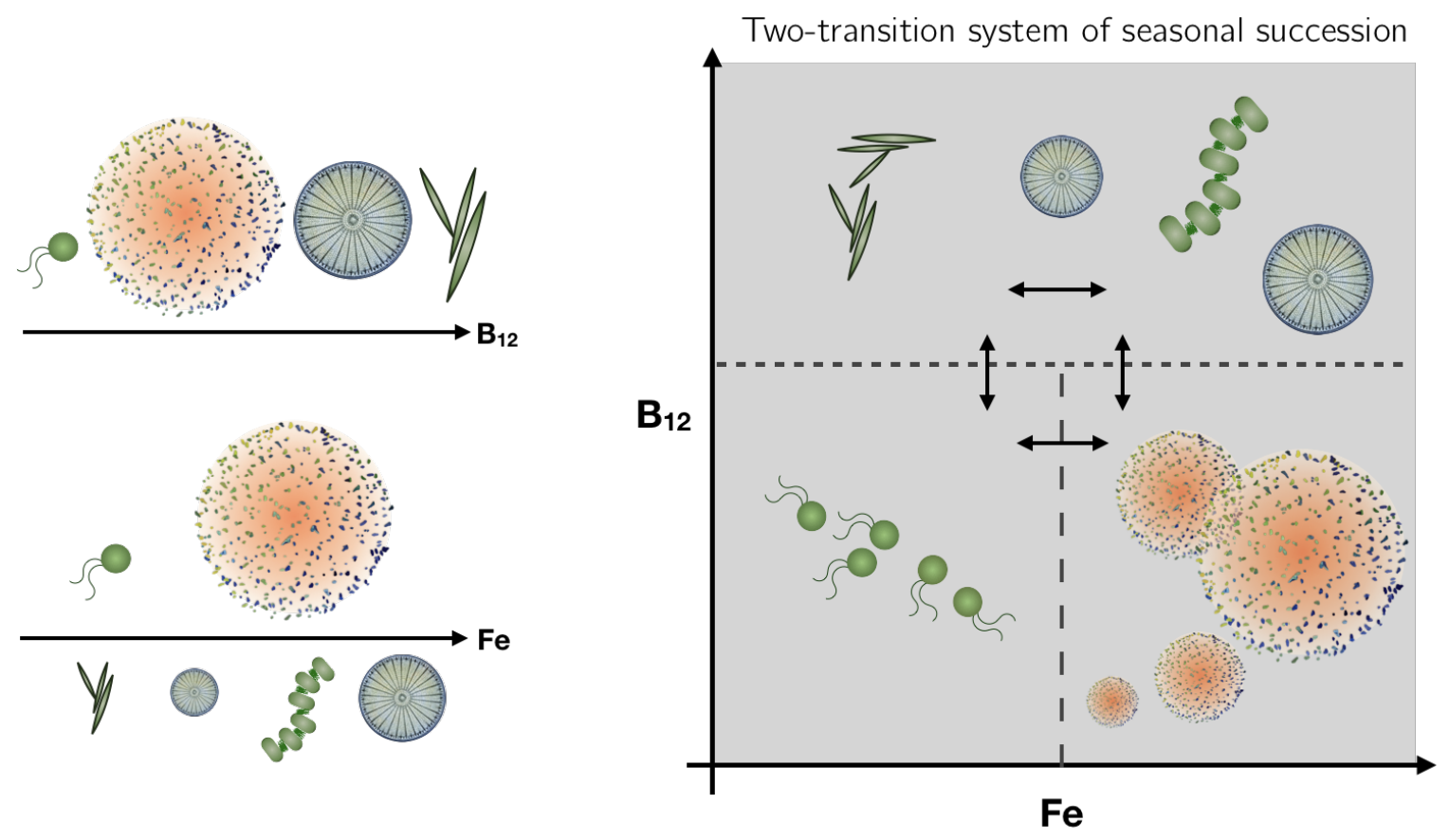

Figure 3-19: A hypothesis for micronutrient-driven seasonal succession in Antarctic coastal marine microbial communities. A two-transition system may explain a shift from early spring single cell P. antarctica into colonies given sufficient iron. The growth and organic carbon produced from $P$. antarctica supports secondary production by bacteria, which then produce $\mathrm{B}_{12}$. Diatoms that are auxotrophic for the vitamin will then be able to grow in the summer due to vitamin-primed waters.

A mechanistic understanding the seasonal succession of phytoplankton species in Antarctic coastal waters remains challenging to substantiate. In the Ross Sea, microbial community succession often starts with $P$. antarctica dominating the spring and early summer in iron-replete conditions $(>1 \mathrm{nM})$, followed by a bloom of bacterioplankton, then a bloom of diatom species. P. antarctica dominates in the early spring blooms, under iron-replete conditions and lower irradiances compared to summer. This has been attributed to $P$. antarctica's neutral buoyancy of the colonies, and its maintaining near-maximal photosynthetic rates under low light (Arrigo et al., 1998, 1999), perhaps owing to the small size of single-cell $P$. antarctica. Recent lab culture experiments demonstrate that $P$. antarctica (strain CCMP 1871) is triggered into colony formation due to higher Fe concentrations (Bender et al., 2018) and Chapter 2.

In the early spring, despite available Fe from an unused winter reserve, vitaminauxotrophic diatoms are $\mathrm{B}_{12}$-limited due to reduced bacterioplankton activity in the winter and thus vitamin production. If $P$. antarctica is not auxotrophic for $\mathrm{B}_{12}$, as the results from Chapter 2 indicate, then it is free from vitamin limitation early in the growing season. $P$. antarctica in its single cell stage has a relatively low iron 
requirement due to its small size. The life cycle transition between two morphotypes with distinct ecophysiologies infers a competitive advantage to $P$. antarctica over diatoms in the early spring, a mechanism discussed more in Chapter 4 and explored in a model. Even if Fe concentrations are higher the early spring, some diatoms may be light-limited and restricted due to their larger size and higher iron quota (Sedwick et al., 2000; Timmermans et al., 2001a). A growing bloom of P. antarctica colonies produces acrylic acid, a bactericide; however, as colonial bloom growth slows to senescence, they are colonized by heterotrophic bacteria that consume the carbon-rich colonial mucus. It has been suggested that bacterioplankton growth on the decaying $P$. antarctica bloom may increase the $\mathrm{B}_{12}$ supply needed for vitamin-auxotrophic diatoms to grow (Bertrand et al., 2007; Ducklow et al., 2001; Sedwick et al., 2000). As field amendment incubation experiments demonstrate, diatoms rapidly respond to $\mathrm{B}_{12}$ additions, even if $P$. antarctica dominates the initial community. Later in the summer and early autumn, low light or iron concentration $(<0.2 \mathrm{nM})$ limit both $P$. antarctica and diatom growth (Goffart et al., 2000). We build on this hypothesis to present a framework of micronutrient limitation by iron and $\mathrm{B}_{12}$ (diagrammed in Fig.3-19), as controls on seasonal succession of $P$. antarctica morphotypes, bacterioplankton, and diatom assemblages.

The seasonal succession of $P$. antarctica in the early spring and summer to diatoms through the end of summer, may be explained by a balance between micronutrient limitation by $\mathrm{Fe}$ and $\mathrm{B}_{12}$ and the life cycle of $P$. antarctica. These lab culture studies in combination with inferences from field studies and observations led to the hypothesis of a two-transition system of micronutrient limitation for the seasonal succession of Antarctic coastal microbial communities. P. antarctica solitary cells dominates early spring blooms in the Amundsen Sea and Ross Sea with increasing sunlight and ample winter reserve of iron, due to its overall smaller size, lower iron requirements, and ability to transition into the colonial life stage with sufficient iron coupled with its flexible $\mathrm{B}_{12}$ requirements, relative to diatoms. That $P$. antarctica can utilize more efficient- $\mathrm{B}_{12}$ metabolic pathways when the vitamin is available further expands is ecological niche and competitiveness due to its metabolic flexibility and multifaceted ecophysiology in its two primary life cycle stages. I extend this line of reasoning to suggest that the seeding of trace metals and initial population from basal sea ice melt may be a more relevant nutrient source for diatoms, due to high concentrations of sea-ice diatom biomass and bioactive trace metals in seasonal sea ice (and potentially high levels of $\mathrm{B}_{12}$ ) (Taylor and Sullivan, 2008), in regions of the Amundsen Sea and Ross Sea polynyas, relative to initial micronutrient input from sediment or ice shelf sources. $\mathrm{B}_{12}$-auxotrophic diatoms may benefit from the $P$. antarctica-bloom terminus due to an increase in $\mathrm{B}_{12}$ concentrations produced by active bacteria feeding on the dying bloom, enabling diatoms to out-compete $P$. antarctica with a vitamin-boosted growth rate. This hypothesis is examined through a numerical ecosystem model in Chapter 4. 


\subsection{Conclusion}

Coastal Antarctic seas are the most productive regions of the Southern Ocean and sustain the entire Antarctic food web, but are also most sensitive to the consequences of climate change (Arrigo et al., 2008, 2012). The Amundsen Sea has some of the highest ice shelf melt rates around Antarctica and documented high seawater particulate iron concentrations originating from basal ice shelves' melting and sediment resuspension, even exceeding 100,000 pmol Fe L ${ }^{-1}$ near the Crosson Ice Shelf in the eastern Amundsen sector and thus an input of dFe as well (Dinniman et al., 2016; Planquette et al., 2013). In particular, the Pine Island and Thwairtes glaciers are rapidly melting into the Amundsen Sea, depositing Fe and (to a lesser extent) Co. The Amundsen region has the highest primary productivity per unit area compared to all other coastal Antarctic regions and is fueled by a large iron supply from upwelling and meltwater inputs that is bioavailable to marine microbes (Thuróczy et al., 2010). This increased iron supply may fuel growth and "tip" communities into limitation by other resources, like $\mathrm{B}_{12}$. In anticipated future conditions due to climate change, the microbial competition and community dynamics should be expected to change (Petrou et al., 2016).

Given the changing dynamics of Antarctic ice shelves in the current and future climate, it is plausible that there is an increased iron supply to surface seawater microbial communities due to warming (basal ice shelf melt, iceberg deposits, basal sea ice melt, and sediment resuspension). In these environmental conditions, $P$. antarctica likely has an ecological advantage, both due to its low iron requirements also to its unique ecophysiology wherein it transitions to its colonial life stage morphotype in the presence of higher available iron (Bender et al., 2018). In its colonial life stage, $P$. antarctica persists as a highly productive seasonal bloom that is minimally grazed. Furthermore, the results from Chapter 2 demonstrate that $P$. antarctica maintains a stable growth rate over a broad range of $\mathrm{B}_{12}$ concentration, even in culture conditions devoid of the vitamin, indicating that it is not auxotrophic for $\mathrm{B}_{12}$, unlike some keystone polar diatom species (Ellis et al., 2017). That P. antarctica does not need $\mathrm{B}_{12}$, but can adjust its metabolism to utilize $\mathrm{B}_{12}$, is consistent with field amendment studies that observe diatoms responding to $\mathrm{B}_{12}$ additions (Bertrand et al., 2011a). However, as Chapter 2 also demonstrated, P. antarctica switches from MetE to MetH in the presence of sufficient $\mathrm{B}_{12}$, and in the case of higher iron and higher $\mathrm{B}_{12}$ concentrations, there is an increased growth rate in the predominantly colonial cultures.

Future studies could address the hypothesis of micronutrient-limited seasonal succession through a seasonal study of iron and cobalamin amendment experiments in natural seawater communities. Additionally, laboratory based culture and co-culture studies can continue to probe the micronutrient requirements and physiological traits of $P$. antarctica and polar diatoms. A useful outcome would be to collect enough data to develop a resource-ratio theory framework to test the seasonal controls on community succession (Tilman, 1981). Lastly, CICLOPS cruise data is still being generated and analyzed, and the information from more trace metal measurements, along with transcriptomic and proteomic data will further enhance the analysis presented in this chapter and can help to elucidate patterns of cobalt and cobalamin stress in natural 
communities. Understanding the drivers of community dynamics in coastal Antarctic waters can enable a predictive framework for not only the microbial community, but impacts that spread across the Antarctic food web from krill, to polar fish, whales, and increasingly humans that are extracting more resources from the Southern Ocean and Antarctic coastal systems.

\section{Author Contributions}

D. Rao and M. Saito designed the experiments and D.R. wrote the manuscript with feedback from co-authors. D.R. conducted the radiotracer incubation experiments and analysis. R. Chimel conducted the cobalt concentration and speciation analysis. N. Schanke and the lab of J. DiTullio conducted the chemotaxonomic pigment analysis and provided guidance on interpretation of these results. M. Follows, S. Dutkiewicz, and S. Saavedra contributed many helpful discussions and feedback on the analysis and manuscript.

\section{Acknowledgements}

The authors are most grateful to the Captain, crew, and ASC support staff of the $R / V$ Nathaniel B. Palmer. A special thanks to Jamee Johnson for her help with the rad van aboard the ship. D.R. thanks Marissa Kellogg, Dawn Moran, and Veronique Oldham for their assistance in sample collection during the CICLOPS cruise. D.R. thanks Pasquale Castagno for feedback on the water mass characterization. D. Rao gratefully acknowledges the funding support of National Science Foundation Office of Polar Program (OPP) grant to G. DiTullio and M. Saito (OPP-1643684, OPP-1643845, and OPP-1644073).

\subsection{Supplemental Materials}

\subsubsection{CICLOPS cruise data access}

CICLOPS cruise data is available via BCO-DMO. Station Maps for the Amundsen, Ross Sea, and Terra Nova Bay sectors can be found under the CTD Hydrography section. 


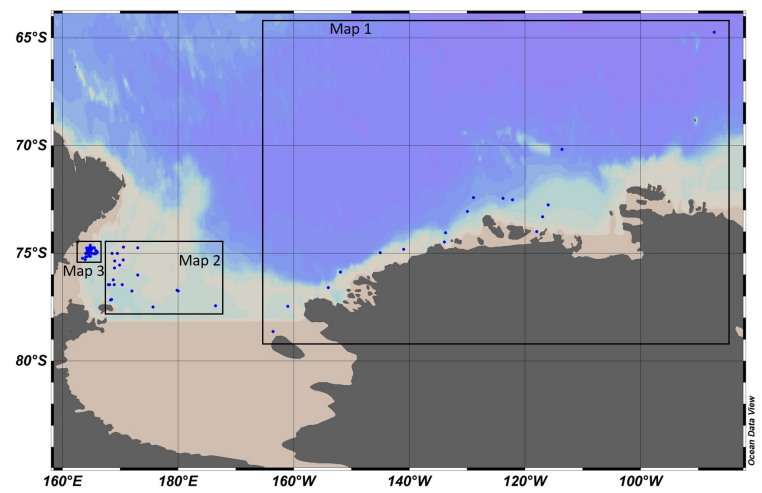

(a) All Stations

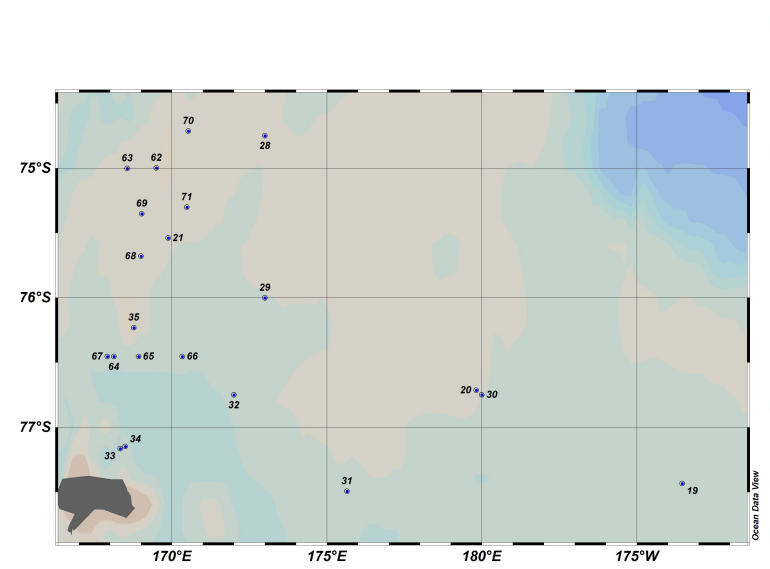

(c) Map 2: Ross Sea

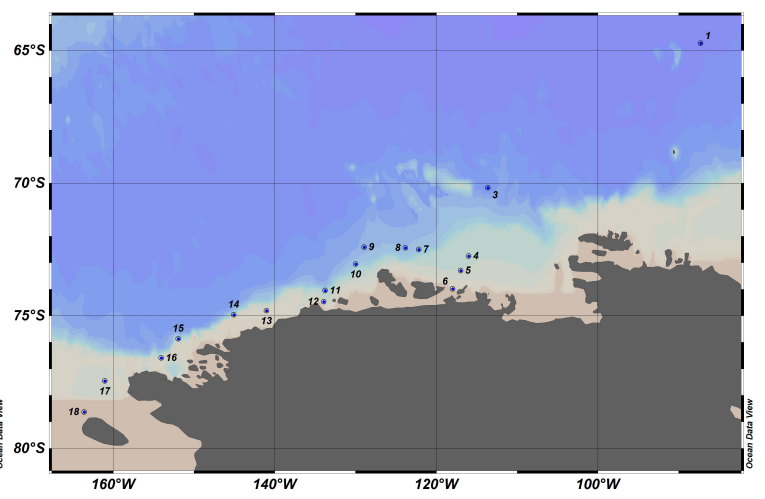

(b) Map 1: Amundsen Sea

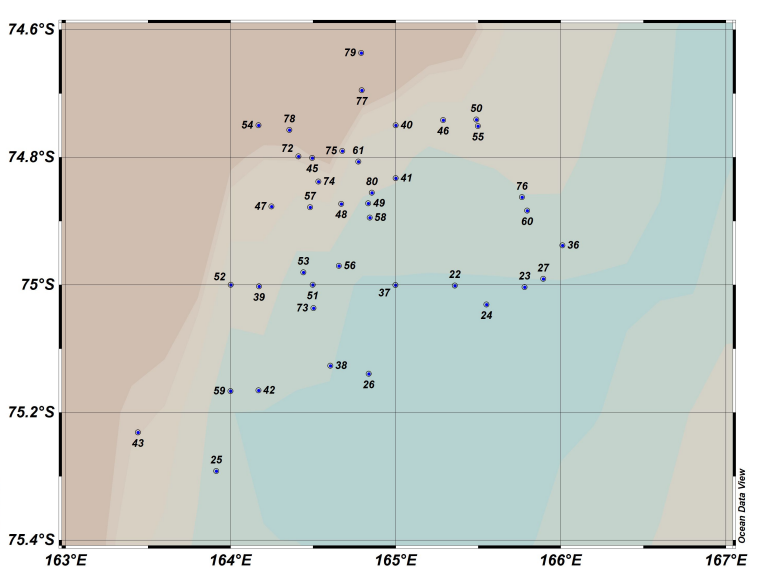

(d) Map 3: Terra Nova Bay

Figure 3-20: CICLOPS stations, grouped by region. Source: BCO-DMO: CTD Hydrography 


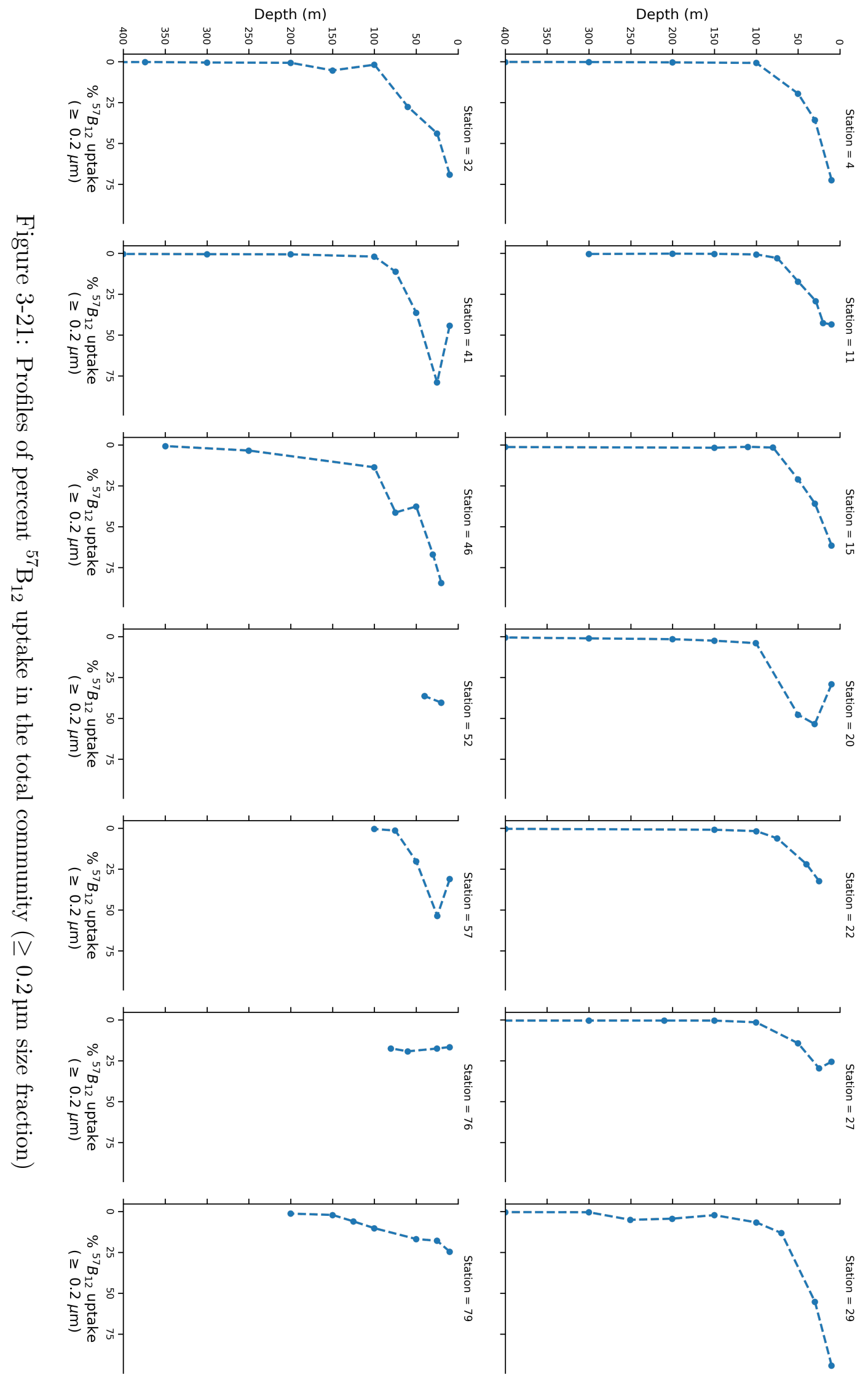




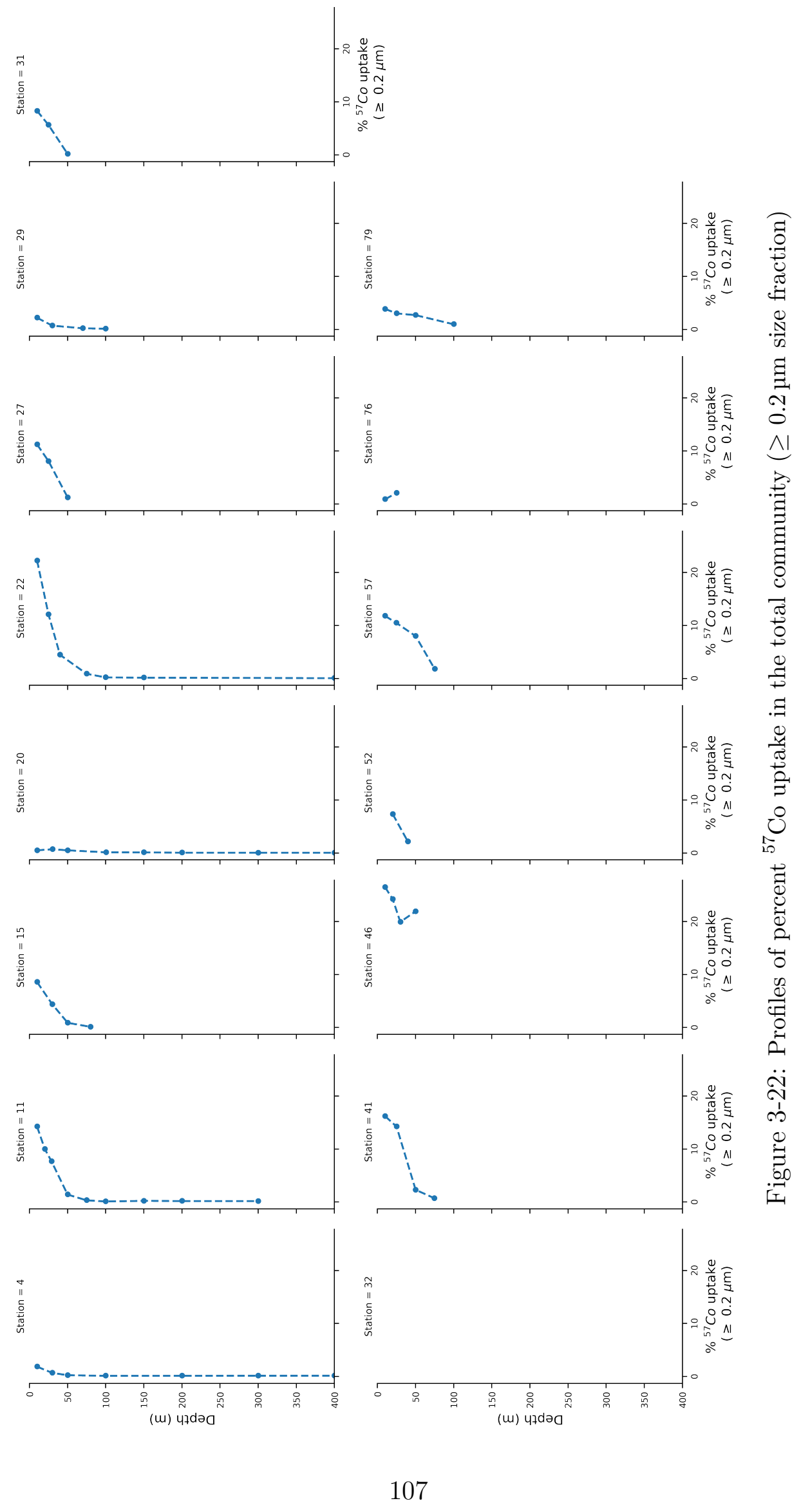




\subsubsection{Cobalt distribution in surface Antarctic coastal waters during CICLOPS}

\begin{tabular}{llll} 
Station Set & All & AR & Surface \\
\hline Total Co, $n=$ & 147 & 67 & 82 \\
Mean(STD) & $36.5(14.2)$ & $37.6(9.43)$ & $31.8(12.2)$ \\
Max & 111.1 & 57.9 & 54.25 \\
\hline Labile Co, $n=$ & 235 & 67 & 119 \\
Mean(STD) & $4.5(6.4)$ & $4.1(3.6)$ & $2.6(4.6)$ \\
Max & 36.1 & 15.8 & 29.5 \\
\hline Labile/Total, $n=$ & 146 & 67 & 81 \\
Mean(STD) & $0.09(0.12)$ & $0.09(0.09)$ & $0.07(0.11)$ \\
Max & 0.51 & 0.41 & 0.48 \\
\hline
\end{tabular}

Table 3.3: Dissolved cobalt values during CICLOPS research cruise. Minimum labile Co values are $0.0 \mathrm{pM}$ or undetectable below instrument precision. STD = standard deviation. AR transect spans TM Stations 10, 11, 15, 20, 22. Surface samples are those $\leq 200 \mathrm{~m}$. The sample number $\mathrm{n}$ is included in parentheses. 


\begin{tabular}{|c|c|c|c|}
\hline Station Set & $\begin{array}{l}\text { Total Co } \\
(\mathrm{pM})\end{array}$ & $\begin{array}{l}\text { Labile Co } \\
(\mathrm{pM})\end{array}$ & $\begin{array}{l}\text { Percent Labile } \\
(\%)\end{array}$ \\
\hline $\begin{array}{l}\text { All stations } \\
\text { mean }(n) \\
\text { range }\end{array}$ & $\begin{array}{l}37.4(n=147) \\
0.0-111.1\end{array}$ & $\begin{array}{l}4.9(n=235) \\
0.0-36.1\end{array}$ & $\begin{array}{l}10.9(n=147) \\
0.0-51.35\end{array}$ \\
\hline $\begin{array}{l}\text { Surface } \\
\text { mean }(n) \\
\text { range }\end{array}$ & $\begin{array}{l}32.8(n=83) \\
0.0-52.3\end{array}$ & $\begin{array}{l}2.77(n=134) \\
0.0-29.4\end{array}$ & $\begin{array}{l}8.0(n=83) \\
0.0-48.1\end{array}$ \\
\hline $\begin{array}{l}\text { Deep } \\
\text { mean }(n) \\
\text { range }\end{array}$ & $\begin{array}{l}43.5(n=64) \\
22.6-111.1\end{array}$ & $\begin{array}{l}7.8(n=64) \\
0.0-36.1\end{array}$ & $\begin{array}{l}14.6(n=64) \\
0.0-51.4\end{array}$ \\
\hline $\begin{array}{l}\text { Amundsen Sea } \\
\text { mean }(n) \\
\text { range }\end{array}$ & $\begin{array}{l}39.8(n=45) \\
21.4-54.3\end{array}$ & $\begin{array}{l}4.3(n=45) \\
0.0-15.8\end{array}$ & $\begin{array}{l}10.6(n=45) \\
0.0-41.2\end{array}$ \\
\hline $\begin{array}{l}\text { Surface } \\
\text { mean }(n) \\
\text { range }\end{array}$ & $\begin{array}{l}39.2(n=26) \\
21.4-54.3\end{array}$ & $\begin{array}{l}5.2(n=26) \\
0.0-15.8\end{array}$ & $\begin{array}{l}12.6(n=26) \\
0.0-41.2\end{array}$ \\
\hline $\begin{array}{l}\text { Deep } \\
\text { mean }(n) \\
\text { range }\end{array}$ & $\begin{array}{l}40.7(n=19) \\
31.9-51.5\end{array}$ & $\begin{array}{l}3.1(n=19) \\
0.0-11.9\end{array}$ & $\begin{array}{l}7.9(n=19) \\
0.0-28.7\end{array}$ \\
\hline $\begin{array}{l}\text { Ross Sea } \\
\text { mean }(n) \\
\text { range }\end{array}$ & $\begin{array}{l}40.6(n=46) \\
9.1-60.1\end{array}$ & $\begin{array}{l}5.8(n=67) \\
0.0-28.3\end{array}$ & $\begin{array}{l}14.5(n=46) \\
0.0-51.4\end{array}$ \\
\hline $\begin{array}{l}\text { Surface } \\
\text { mean }(n) \\
\text { range }\end{array}$ & $\begin{array}{l}34.0(n=26) \\
9.1-52.1\end{array}$ & $\begin{array}{l}2.8(n=39) \\
0.0-15.6\end{array}$ & $\begin{array}{l}9.3(n=26) \\
0.0-35.2\end{array}$ \\
\hline $\begin{array}{l}\text { Deep } \\
\text { mean }(n) \\
\text { range }\end{array}$ & $\begin{array}{l}49.2(n=20) \\
32.6-60.1\end{array}$ & $\begin{array}{l}9.9(n=28) \\
0.3-28.3\end{array}$ & $\begin{array}{l}21.2(n=20) \\
2.8-51.4\end{array}$ \\
\hline $\begin{array}{l}\text { Terra Nova Bay } \\
\text { mean }(n) \\
\text { range }\end{array}$ & $\begin{array}{l}32.9(n=56) \\
0.0-111.1\end{array}$ & $\begin{array}{l}4.7(n=123) \\
0.0-36.1\end{array}$ & $\begin{array}{l}8.2(n=56) \\
0.0-48.1\end{array}$ \\
\hline $\begin{array}{l}\text { Surface } \\
\text { mean }(n) \\
\text { range }\end{array}$ & $\begin{array}{l}26.3(n=31) \\
0.0-52.9\end{array}$ & $\begin{array}{l}1.9(n=69) \\
0.0-29.4\end{array}$ & $\begin{array}{l}3.2(n=31) \\
0.0-48.1\end{array}$ \\
\hline $\begin{array}{l}\text { Deep } \\
\text { mean }(n) \\
\text { range }\end{array}$ & $\begin{array}{l}41.2(n=25) \\
22.6-111.1\end{array}$ & $\begin{array}{l}8.3(n=54) \\
0.0-36.1\end{array}$ & $\begin{array}{l}13.4(n=25) \\
0.0-39.8\end{array}$ \\
\hline
\end{tabular}

Table 3.4: Dissolved cobalt dCo values during CICLOPS cruise. Dissolved cobalt dCo values including total cobalt, labile cobalt, and percent labile cobalt. Minimum labile dCo values are $0.0 \mathrm{pM}$ or undetectable below instrument precision. Surface samples are those $\leq$ $200 \mathrm{~m}$, and deep samples are $>200 \mathrm{~m}$. The sample number $n$ is included in parentheses. 


\section{Appendix 3.A Ecological cobalt and cobalamin cycles in Antarctic marginal seas}

The biogeochemistry of cobalt (Co) in marine systems is driven by environmental inputs and microbial biochemical transformations, and is succinctly reviewed in (Saito et al., 2004). Given that upper continental crust has a Co:Fe of roughly 1:2600 (10 ppm vs. $2.6 \times 10^{4} \mathrm{ppm}$ ) overall cobalt inputs are scant, even relative to trace iron fluxes (Carr and Turekian, 1961; Taylor and McLennan, 1985). In the open ocean, dissolved cobalt (dCo) concentrations are low, typically $\leq 120$ pM (Swanner et al., 2014). In the geochemical cycle, "new" dCo enters seawater through crustal input at the surface (aeolian deposition, riverine input, surface glacial deposits via meltwater input) or at depth (release from anoxic sediments in oxygen-minimum zones (Saito et al., 2004; Sundby et al., 1986), or input via hydrothermal activity). ${ }^{9}$ In the Antarctic coastal seas, other important sources of cobalt include sediment resuspension, basal sea ice melt, and basal ice shelf melt (Noble et al., 2013). Here, I review the main mechanisms driving the biogeochemical cobalt cycling in Antarctic coastal environments.

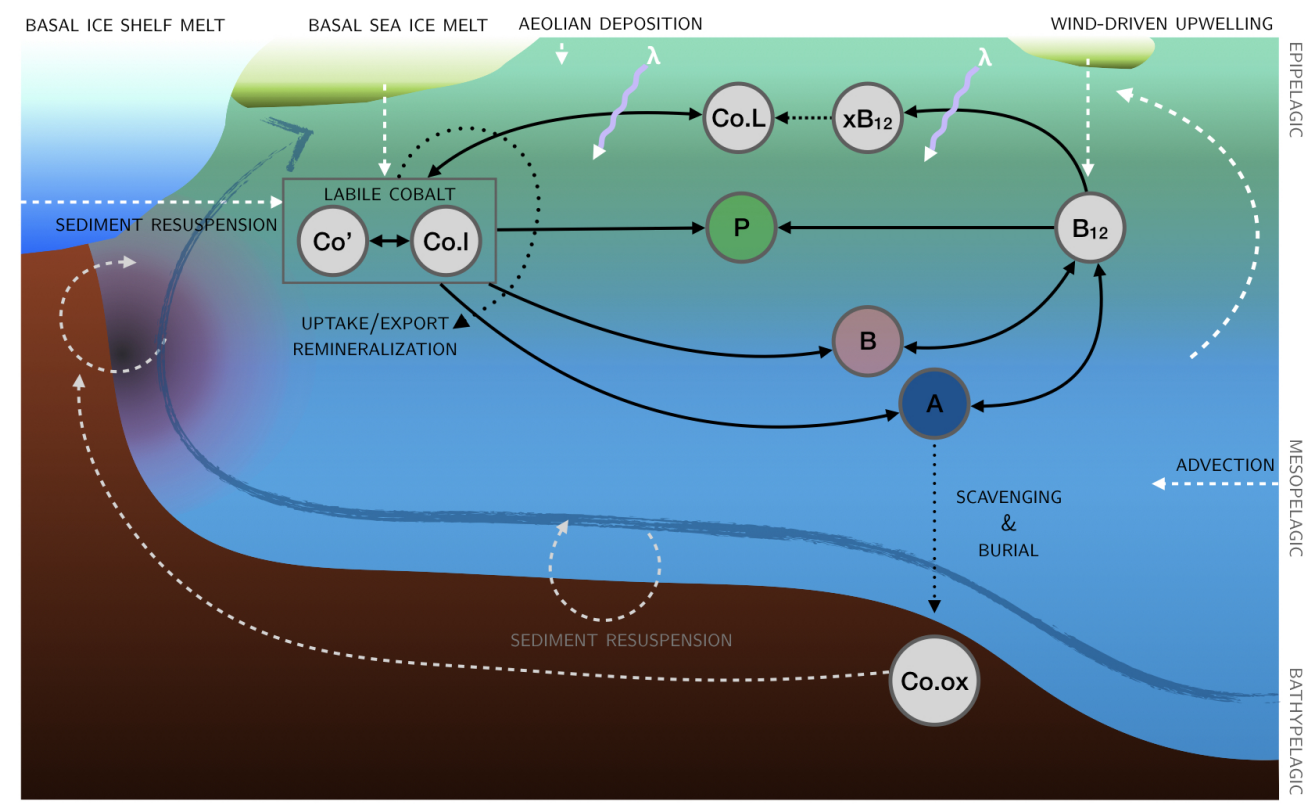

Figure 3-23: The Antarctic marine biogeochemical cobalt cycle with an emphasis on ecological cobalt cycling between cobalt species and cobalamin.

Coastal shelf areas like the Amundsen Sea, Ross Sea, and Terra Nova Bay are $<500 \mathrm{~m}$ in depth, with deeper areas extending to $1,000+\mathrm{m}$ in trenches near the ice shelves and continental shelves (Dinniman et al., 2016; Planquette et al., 2013). The input of trace metals to the upper water column from metal-rich bottom water (e.g.,

\footnotetext{
${ }^{9}$ Aeolian (dry) deposition of Co is considered a minor source, representing $\leq 2 \%$ of concentrations measured in the mixed layer. One study measured the dissolution of cobalt from a natural dust event that contributed $0.01-0.43 \mathrm{pM}$ dCo enrichment to the mixed layer (Thuróczy et al., 2010).
} 
particulate and dissolved iron), sea ice and overlying snow melt, melting icebergs calved from ice shelves, and glacial meltwater are important sources of micronutrients to local microbial communities (Noble et al., 2013; Sedwick et al., 2000). Meltwater from the Amundsen Sea ice shelves forms a westward surface flow along the Antarctica coastal current into the Ross Sea, where it combines with Ross Sea ice shelf melt waters. These meltwater flows are important advective supplies of trace metals and micronutrients (Dinniman et al., 2016). Glacial ice derives its metal content from mineral aerosols (dust) trapped in the ice, lithogenic material, and marine sediments that are scoured by the basal glacial ice as it is advected into the polynyas. These meltwater sources would have a pronounced effect on marine microbial communities during the late spring and early summer when melt rates are highest.

Ecological cycling of dissolved cobalt in marine systems involves the uptake, transformation, and exchange of dCo in various biochemical forms, but predominantly as labile $\mathrm{Co}\left(\mathrm{Co}_{\text {labile }}\right)$ and cobalamin. Cobalt leaves the surface ocean in sinking particles that were accumulated in biomass or other particulate matter. Dissolved cobalt remains in the surface via strong organic ligands of unknown structure, though it is speculated that some of these ligands are likely portions of the $\mathrm{B}_{12}$ molecule (Saito et al., 2004). The bioavailability of Co depends on its chemical speciation, with $\mathrm{Co}_{\text {labile }}$ (operationally defined as Co' and weakly complexed Co) being the most bioavailable. Marine microbes both actively and passively cycle dCo, resulting in cobalt typically having a hybrid-type profile: with marine microbes assimilating $\mathrm{dCo}$ into biomass, resulting in a nutrient-like profile in the upper ocean, and scavenging with depth. Actively, both phytoplankton and bacterioplankton uptake Co for use in metalloenzymes and in the form of cobalamin. Passively, manganese-oxidizing bacteria co-oxidize cobalt, scavenging throughout the water column that often results in the hybrid-type profile with depth in tropical and subtropical waters (Moffett and Ho, 1996; Saito et al., 2004; Tebo et al., 1984). However, in Antarctic waters (specifically the Ross Sea and likely other regions) cobalt scavenging by Mn-oxidizing bacteria is minimal, and a nutrient-like profile is more typically observed (Saito et al., 2010).

Cobalt is a bio-essential micronutrient for marine microbes. Some phytoplankton contain a cambialistic (biochemical substitution of metals without loss of function) $\mathrm{Zn}-\mathrm{Co}$ carbonic anhydrase (DCA1), an adaptation for survival in micronutrientdepleted surface ocean waters (Saito and Goepfert, 2008). Several centric marine diatoms (Thalassiosira weissflogii, T. oceanica, and T. pseudonana) show metal substitution of either Co or Cd for Zn (Price and Morel, 1990; Sunda and Huntsman, 1995). Notably, P. antarctica contains a Zn-Co carbonic anhydrase and preferentially substitutes Zn; whereas the sea-ice diatom Chaetoceros calcitrans cannot substitute zinc for cobalt, but still actively assimilates dCo (Timmermans et al., 2001b). Phaeocystis cultures provide evidence of extracellular carbonic anhydrase that may allow them to maintain high carbon uptake capability in bloom conditions (where $\mathrm{dCO}_{2}$ can be limiting), resulting in elevated $\mathrm{pH}$; growth has even been observed in conditions of $<150$ ppm $\mathrm{CO}_{2}$ (Elzenga et al., 2000; Schoemann et al., 2005; Tortell et al., 2008). Furthermore, P. antarctica has a lower Co requirement than some diatoms (e.g., Thalassiosira pseudonana) (Saito and Goepfert, 2008). These differences in metabolic 
pathways can manifest in consequential competition dynamics in a micronutrient limited ecosystems in Antarctic marginal seas.

Cobalamin is arguably the most important cobalt-containing molecule in marine systems. $\mathrm{B}_{12}$ is a complex metallo-organic compound with a cobalt atom positioned in a corrin ring structure. The biosynthesis of $\mathrm{B}_{12}$ requires acquiring extracellular Co (Martens et al., 2002). Bacterioplankton (pelagic bacteria and archaea) uptake $\mathrm{dCo}$ and some synthesize cobalamin, while arguably all uptake cobalamin even if they have the biosynthetic capability. Only bacterioplankton can synthesize $\mathrm{B}_{12}$ de novo; and most eukaryotic phytoplankton are auxotrophic for $\mathrm{B}_{12}$, requiring an exogenous supply (Croft et al., 2005). Most eukaryotic phytoplankton require $\mathrm{B}_{12}$ for protein and nucleic acid synthesis, as used in $\mathrm{B}_{12}$-dependent methionine synthase $(\mathrm{MetH})$ and ribonucleotide reductase. Algae either contain MetH or maintain both MetH and the $\mathrm{B}_{12}$-independent form (MetE) that is less efficient. The $\mathrm{B}_{12}$ transporter in diatoms has been identified (CBA1), but the uptake mechanisms for most eukaryotes remains elusive (Bertrand et al., 2012). Bacteria assimilate $\mathrm{B}_{12}$ through ABC transporters and TonB-dependent receptor protein systems (Klebba et al., 1993; Rodionov et al., 2003; Vitreschak et al., 2003). In Antarctic systems, $\mathrm{B}_{12}$ supply from the bacterial community is likely lower than other oceanographic regions, due to low and variable rates of secondary production (Ducklow et al., 2001), limited Co inputs from terrestrial sources, lack of cyanobacteria (main producers of pseudocobalamin in non-polar waters), and potentially high UV-degradation due to the austral spring ozone hole (Crutzen, 1992).

Cobalamin is removed from the surface ocean via sinking biomass and photochemical degradation by ultraviolet radiation disrupts the complex structure of $\mathrm{B}_{12}$. The resulting vitamin degradation products may come in a variety of forms, including intact corrin-rings with no lower ligand or labile Co released from the corrin ring structure. It is hypothesized that these cobalamin-degradation products constitute the strong organic ligands that bind cobalt in the surface ocean (Saito et al., 2004). Although less bioavailable, these natural ligands may result in Co retention in surface waters, enabling later $\mathrm{dCo}$ or partial $\mathrm{B}_{12}$ molecules to be taken up and remodeled by microbes that contain the relevant biochemical pathways (Helliwell et al., 2016). Ultimately, it is through ecological cycling within the microbial loop that Co transforms between chemical species of varying bioavailability in coastal Antarctic marine systems. 


\section{Appendix 3.B Water mass characterization of CICLOPS expedition samples}

In order to contextualize the patterns of cobalt and cobalamin uptake, we examine the water mass characteristics of the Antarctic shelf waters that were sampled during CICLOPS. This appendix includes the physio-chemical criteria used to determine water masses encountered during the CICLOPS research expedition at the sample locations for data presented in Chapter 2. Figure 3-26 shows the Temperature-Salinity (TS) diagram subset for all surface stations $(\leq 200 \mathrm{~m})$. Fig.3-3 highlights the dominant Antarctic coastal water masses across the Amundsen Sea, Ross Sea, and Terra Nova Bay, following neutral density $\left(\gamma^{N}\right)$, potential temperature $(\theta)$, and salinity (S) criteria highlighted in Fig.3-25 and Table 3-24 (Castagno et al., 2019; Jackett and McDougall, 1997; Mattson et al., 2012; Orsi and Wiederwohl, 2009). The interaction of these water masses influence the heterogeneous and complex Antarctic coastal surface seascapes in which seasonal phytoplankton blooms develop. The characteristics of these water masses reflect the physical and chemical processes that modify nutrient availability for the marine microbial community, notably meltwater inputs from sea ice, glaciers, and ice shelves, which are important sources of bioessential trace metals (Noble et al., 2013; Planquette et al., 2013).

The Amundsen Sea, Ross Sea, and Terra Nova Bay are distinct hydrographic regions that are influenced by local oceanographic currents and circulation, interactive sea-ice dynamics, meltwater sources, wind-driven processes, and other physical forcings. The Amundsen Sea is characterized by three primary water masses: Antarctic Surface Water (AASW), Circumpolar Deep Water (CDW), and modified Circumpolar Deep Water (mCDW), as defined by their neutral density values (Jackett and McDougall, 1997; Mattson et al., 2012). The majority of samples on the continental slope of the Amundsen Sea had signatures of AASW, AASW Summer, and mCDW waters. The Ross Sea is characterized by additional distinct water masses: Shelf Water (SW), modified Shelf Water (mSW), and Low and High Salinity Shelf Water (LSSW, HSSW), and Ice Shelf Water (ISW), along with mCDW and AASW (Mattson et al., 2012; Orsi and Wiederwohl, 2009). SW is near the freezing point, and so salinity becomes an important criteria to define water masses in this region (Talley, 2011). Terra Nova Bay, the "ice factory" of the Ross Sea, has a strong imprint of sea-ice formation and the HSSW and ISW, the latter is colder than $-1.95^{\circ} \mathrm{C}$ and reflects an interaction with the base of a nearby ice shelf (Orsi and Wiederwohl, 2009). The surface samples in the Ross Sea and Terra Nova Bay have signatures of AASW, AASW Summer, mCDW, $\mathrm{mSW}$, and LSSW/HSSW. Interested readers are encouraged to examine the diagrams in this appendix and read the description of regional hydrography in Chapter 2. 


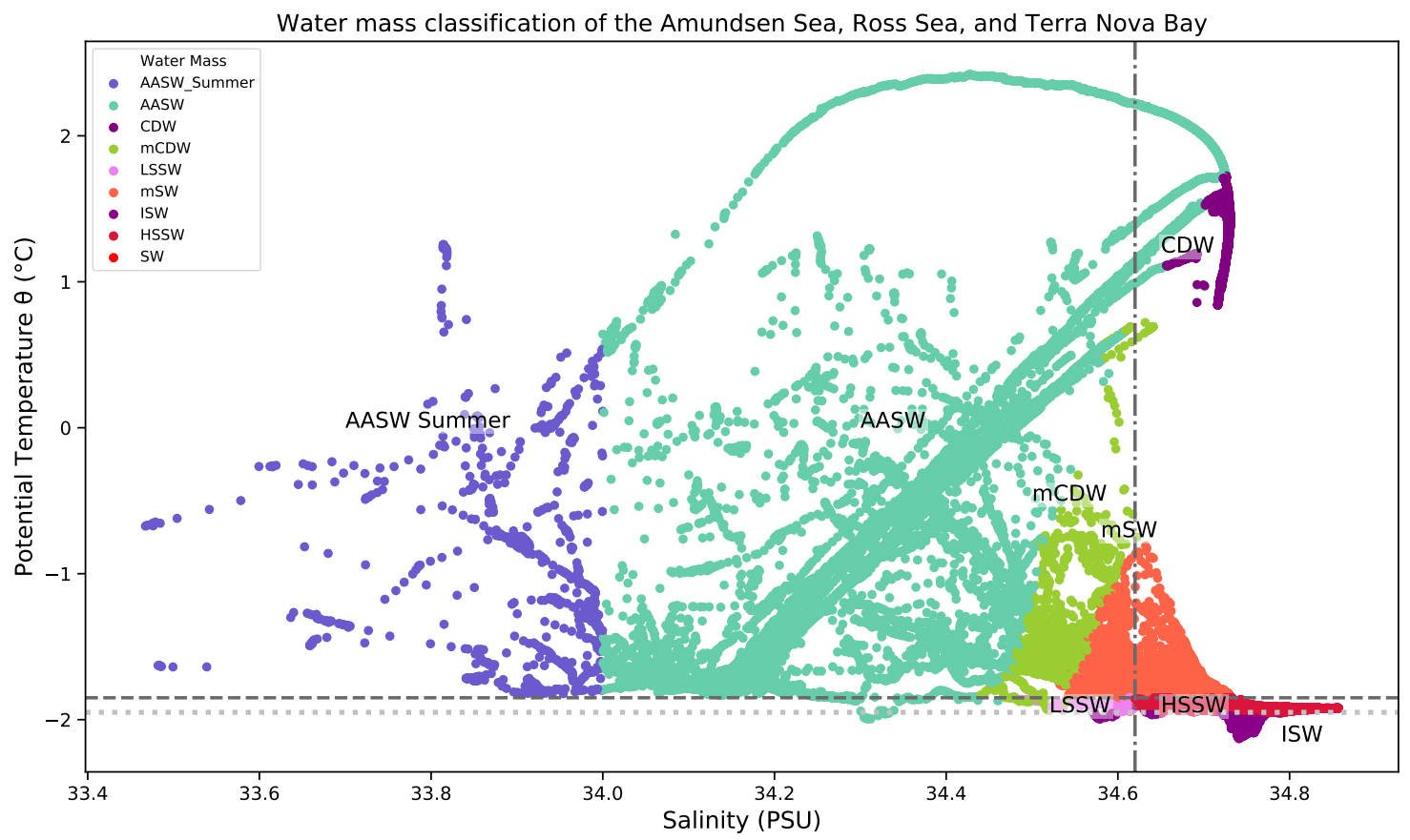

(a) Water masses assigned by criteria in Table 3-24b.

\begin{tabular}{llll}
\hline Water Mass & $\begin{array}{l}\text { Neutral Density } \\
\gamma^{N}\left(\mathrm{~kg} \mathrm{~m}^{-3}\right)\end{array}$ & $\begin{array}{l}\text { Potential Temperature } \\
\theta\left({ }^{\circ} \mathrm{C}\right)\end{array}$ & $\begin{array}{l}\text { Salinity } \\
S(\mathrm{PSU})\end{array}$ \\
\hline CDW & $>28.03$ & $>0.8$ & \\
mCDW & $28.03<\gamma^{N}<28.27$ & & \\
AASW & $<28.03$ & & $<34$ \\
AASW Summer & $<28.03$ & $<-1.85$ & \\
SW & $>28.27$ & $>-1.85$ & $<34.62$ \\
mSW & $>28.27$ & $<-1.85$ & $>34.62$ \\
LSSW & $>28.27$ & $<-1.85$ & \\
HSSW & $>28.27$ & $<-1.95$ & \\
ISW & $>28.28$ & & \\
\hline
\end{tabular}

(b) Antarctic water mass characterization criteria.

Figure 3-24: (a) Water mass characterization criteria applied to CICLOPS CTD data. (b) Criteria for the Antarctic continential slop water mass characterization are summarized from (Castagno et al., 2019; Dinniman et al., 2016; Mattson et al., 2012; Orsi and Wiederwohl, 2009). 


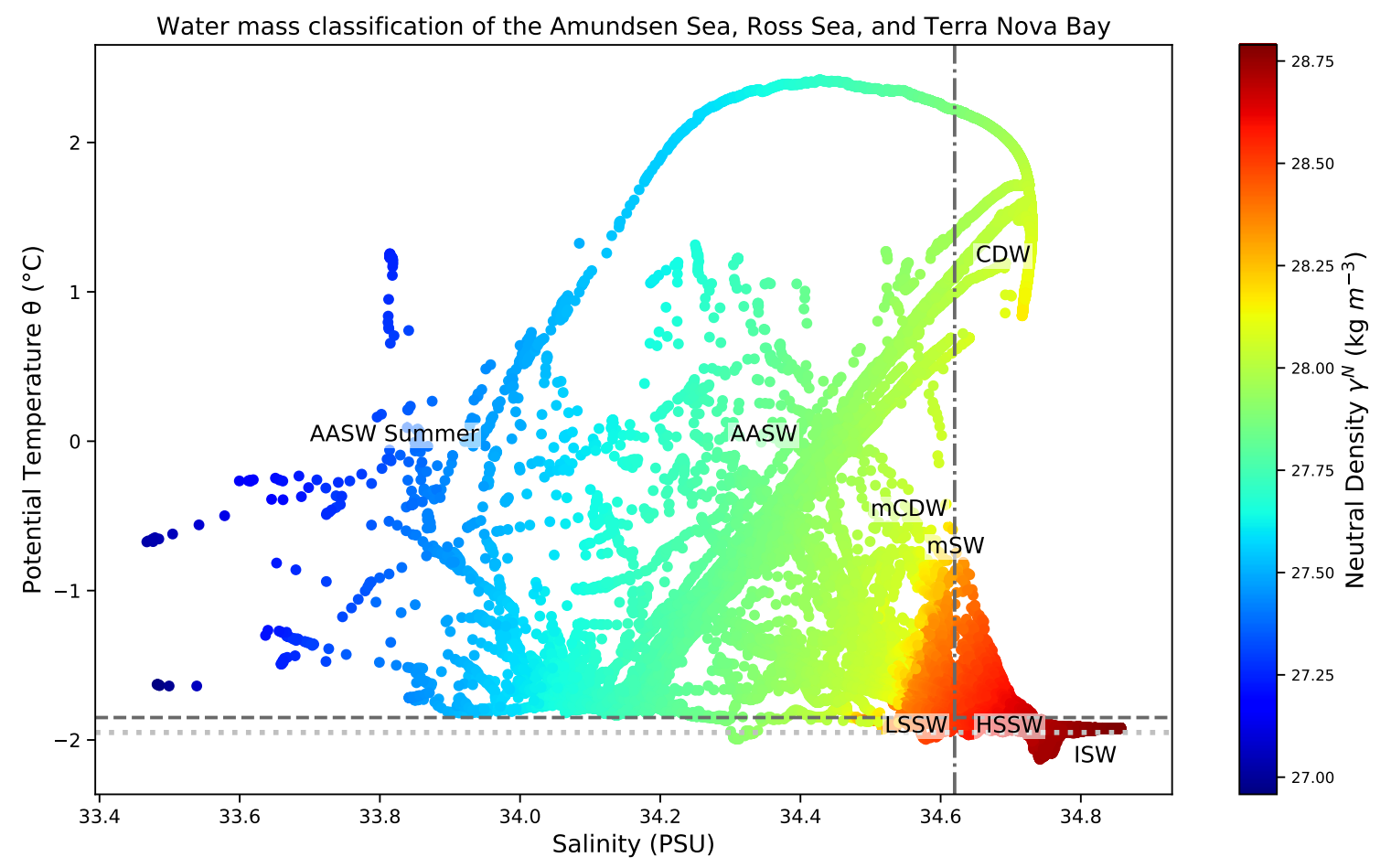

Figure 3-25: The Temperature-Salinity relationship of samples from the Amundsen Sea, Ross Sea, and Terra Nova Bay. The labels in the T-S diagram show the major water masses across these coastal Antarctic regions: modified Circumpolar Deep Water (mCDW/CDW), Antarctic Surface Water (AASW), modified Shelf Water (mSW/SW), Low and High Salinity Shelf Water (LSSH/HSSW), and Ice Shelf Water (ISW). The color of the dots shows neutral density $\left(\gamma^{N}\right)$. The additional lines demarcate water mass properties: grey-dashed horizontal line is between $\mathrm{mSW} / \mathrm{SW}$, silver-dotted horizontal line for ISW/SW, and the grey-dash-dot vertical line for LSSW/HSSW. Water masses are characterized according to neutral density, potential temperature, and salinity (Castagno et al., 2019; Jackett and McDougall, 1997; Mattson et al., 2012; Orsi and Wiederwohl, 2009). 


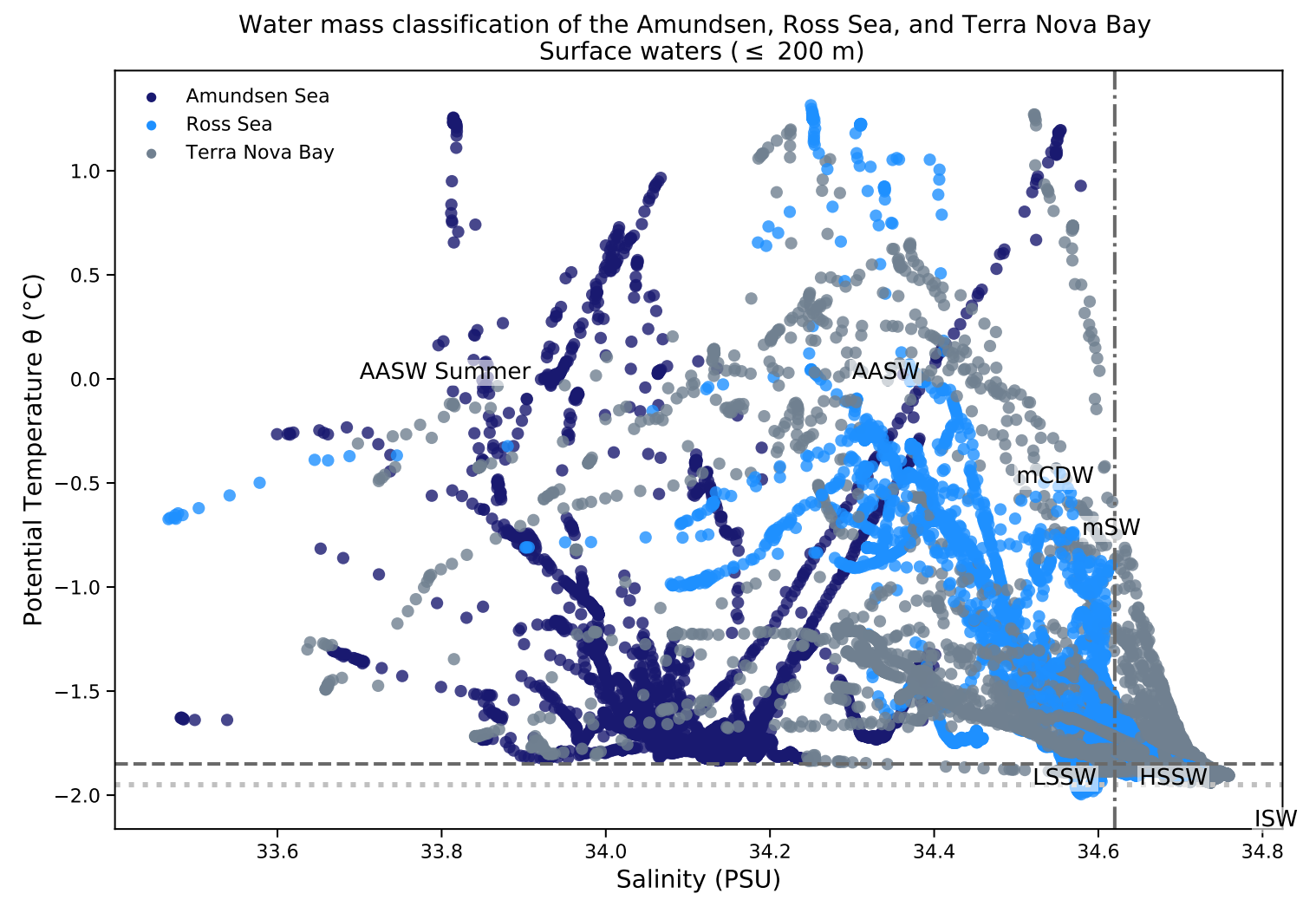

Figure 3-26: The Temperature-Salinity relationships for the surface waters $(\leq 200 \mathrm{~m})$ of the Amundsen Sea, Ross Sea, and Terra Nova Bay. The labels in the T-S diagram show the major water masses across these coastal Antarctic regions: modified Circumpolar Deep Water (mCDW/CDW), Antarctic Surface Water (AASW), modified Shelf Water (mSW/SW), Low and High Salinity Shelf Water (LSSH/HSSW), and Ice Shelf Water (ISW). The data are colored according to region: Amundsen Sea (dark blue), Ross Sea (medium blue), and Terra Nova Bay (grey). The additional lines demarcate water mass properties: grey-dashed horizontal line is between $\mathrm{mSW} / \mathrm{SW}$, silver-dotted horizontal line for ISW/SW, and the grey-dash-dot vertical line for LSSW/HSSW. Water masses are characterized according to neutral density, potential temperature, and salinity (Castagno et al., 2019; Jackett and McDougall, 1997; Mattson et al., 2012; Orsi and Wiederwohl, 2009). 


\section{Chapter 4}

\section{A model of micronutrient-driven seasonal succession of Antarctic marine microbial communities}

"Nothing is less real than realism. Details are confusing. It is only by selection, by elimination, by emphasis, that we get at the real meaning of things." — Georgia O' Keeffe ${ }^{1}$

\section{Context}

The work in this chapter was done in collaboration with Dr. Dutkiewicz and Prof. Follows and consultation with Dr. Saito. I thank them all for their guidance in model development and implementation.

\footnotetext{
${ }^{1}$ Georgia O'Keeffe, "I Can’t Sing So I Paint", New York Sun 5 December 1992. Reproduced in Stremmel, K. (2006), Realism. Köln: Taschen.
} 


\section{Abstract}

Antarctic continental shelf waters are regions of immense primary productivity. Here we ask what drives the seasonal succession of the dominant bloom-forming phytoplankton and bacterioplankton and present a trait-based model of Antarctic marine microbes driven by micronutrients and cross-feeding interactions. Specifically, we ask how a balance between iron and vitamin $\mathrm{B}_{12}$ may drive the observed succession in the Antarctic coastal environments from solitary cells to colonies of Phaeocystis antarctica, followed by bacteria, and then diatoms. In this model, the transition between morphotypes expands the fundamental niche of $P$. antarctica, with each life stage having a unique fitness advantage due to their distinct ecophysiologies. The buildup of organic carbon from an early season $P$. antarctica bloom supports the rise of a bacterial population that synthesizes $\mathrm{B}_{12}$. Larger diatoms may not be competitive against small solitary $P$. antarctica cells for iron, but can have a niche due to $\mathrm{B}_{12}$. We find a dynamic equilibrium between all three model microbial types because each group is primarily limited by a different resource and are interdependent on organic byproducts from the other populations. When driven by a seasonal iron supply, the model can capture the seasonal succession pattern of $P$. antarctica morphotypes, bacteria, and $\mathrm{B}_{12}$-auxotrophic diatoms observed in this region. This model is a proof of concept that organic resources can influence phytoplankton community dynamics, along with a baseline competition for inorganic resources like iron, and enable coexistence and increased diversity. Furthermore, it provides a testable hypothesis for micronutrientdriven seasonal succession of coastal Antarctic marine microbial communities.

\subsection{Introduction}

Antarctic coastal marine ecosystems are regions of immense primary productivity and carbon export from the atmosphere due to the biological pump in combination with the solubility pump. Phytoplankton are the base of the entire Antarctic food web, supporting the growth of marine microbes, krill, fish, penguins, seabirds, seals, whales, and nowadays humans due to increased krill harvest and fishing. In coastal Antarctic waters, massive phytoplankton blooms are a seasonal phenomenon. The blooms often occur along the coastline, ice shelves, and seasonal open-water polynyas. While Antarctic coastal ecosystems are seasonally variable and heterogeneous in nature, there is a regularity observed in the pattern of blooming phytoplankton and heterotrophic bacteria in the spring through summer.

The Amundsen Sea Polynya (ASP) and Ross Sea Polynya (RSP) are two of the beststudied Antarctic coastal ecosystems with recurrent seasonal phytoplankton blooms. These phytoplankton community are dominated by the colony-forming haptophyte Phaeocystis antarctica and an assemblage of diatoms (Arrigo et al., 1999). Typically, $P$. antarctica forms expansive blooms as their colony morphotype. The bloom initiates in the early austral spring (late October through December), when irradiance increases

and seawater is relatively rich in iron $(\mathrm{Fe})$ as a result of the winter reserve and increased glacial and sea-ice melt (Sedwick et al., 2000). These blooms can even persist through 
summer (December to late February). Figure 4-1 describes the seasonal succession of morphotypes, from predominantly solitary, flagellated cells to colonies at the peak of P. antarctica bloom biomass (Smith et al., 2003; Zingone et al., 2011). Following $P$. antarctica is a bacterioplankton bloom, perhaps growing on the vast amounts of organic carbon produced by the colonies (Ducklow et al., 2001). Finally, a variety of diatoms grow through the summer (December through late February), for example $P$. subcurvata and Fragilariopsis curta are two diatom species documented to bloom after P. antarctica (Arrigo et al., 1999; Leventer and Dunbar, 1996; Smith Jr et al., 2000b). Phytoplankton in this system have been demonstrated to be primarily limited by light and iron (Coale et al., 2003; Martin, 1990; Sedwick et al., 2000), but can also be coand secondarily-limited by cobalamin (vitamin $\mathrm{B}_{12}$ ), a trace organic only synthesized by bacterioplankton (Bertrand et al., 2007). Antarctic coastal ecosystems are hot spots of seasonal primary production and biological carbon export from the atmosphere, and are greatly impacted by the composition of the phytoplankton community.

In Antarctic coastal seas, iron and irradiance (and vertical mixing) are assumed to be the primary bottom-up controls on phytoplankton primary productivity, community composition, and biogeography (Arrigo et al., 1998b, 2003; Coale et al., 2003; Sedwick et al., 2007, 2000; Smith et al., 2003; Smith Jr et al., 2000a; Tagliabue et al., 2012). These seas are on the continental shelf in relatively shallow areas, mostly less than $500 \mathrm{~m}$ deep. The local hydrography and sources of dissolved iron are important to consider when trying to understand regional bloom dynamics. Although modeling studies have examined the relative importance of these difference iron sources on primary production (Death et al., 2014; Lancelot et al., 2009; Laufkötter et al., 2018; Tagliabue et al., 2009; Wadley et al., 2014; Wang et al., 2014), large uncertainties remain regarding the timing, magnitude, and variation of iron supply (De Jong et al., 2012; Tagliabue et al., 2016). Dissolved iron is supplied to surface phytoplankton communities from several sources: vertical mixing of deep, iron-rich waters into the euphotic zone (McGillicuddy et al., 2015), melting from ice shelves (subglacial melting) (Gerringa et al., 2012; St-Laurent et al., 2019), icebergs (Raiswell et al., 2016; Smith et al., 2007), surface runoff (Hodson et al., 2017), and iron-enriched sea ice (Lancelot et al., 2009; Lannuzel et al., 2010; Sedwick and DiTullio, 1997), dust deposition (Ito and Kok, 2017; Ito et al., 2019; Tagliabue et al., 2009; Wagener et al., 2008), and sediment resuspension and dissolution (Ardelan et al., 2009; St-Laurent et al., 2019; Tagliabue et al., 2009). Models and observations agree that sediment-derived iron via upwelling is an important deep-water source that can be transported onto the continental shelf area, and that glacial sources dominate surface iron inputs during spring and summer. These two main iron sources have different timescales and relative importance with the seasons. Upwelling of subsurface waters near the slope front is most important during the winter and less so during the stratified summer (Tagliabue et al., 2014). Meltwater iron supply is likely most important in the spring and summer, which can relate dust-aerosol iron in the sea ice, sediment-derived iron from coastal areas, and basal glacial ice that contains lithogenic iron. Iron input to the surface is expected to be highest in the winter and early spring due to deep vertical mixing and a buildup of iron, and reduction in the the summer due to shallowed mixed layers (approximately $100 \mathrm{~m}$ to $25 \mathrm{~m}$ ) combined with increased microbial uptake, export, and 
scavenging (Sedwick et al., 2000). Proximity to meltwater sources is also a crucial factor, where mid-polynya waters may be depleted of dissolved iron, but diatom blooms can persist near the melting ice edge that is iron-enriched (Sedwick and DiTullio, 1997). Both the glacial and deep-water iron source are projected to increase in the future due to climate change. In particular, glacial iron input may be much greater than previously accounted for and is on the rise (Annett et al., 2015; Hawkings et al., 2014, 2018; Shaw et al., 2011).

The "meltwater pump" of iron from glacial sources and buoyancy-driven circulation is a potentially key, yet hitherto unconstrained iron supply mechanism in the highly productive coastal regions and polynyas (Annett et al., 2017; Death et al., 2014; Hodson et al., 2017; St-Laurent et al., 2017, 2019). Iron-rich meltwater from upwelling and meltwater in the coastal and continental slope systems, along with water column stabilization due to the warm cap of meltwater, may drive the productive seasonal blooms in coastal Antarctic regions (Dinniman et al., 2012; Gerringa et al., 2012; Mitchell and Holm-Hansen, 1991; Mitchell et al., 1991). Regions of glacial meltwater often correspond to increased primary production, as seen the fjords and bays of the Antarctic Peninsula, in the Arctic, and the ASP (Ducklow et al., 2007; St-Laurent et al., 2017). The ASP is of particular interest to this dissertation, given the fieldwork conducted in Chapter 3 and encounter of an expansive $P$. antarctica bloom in mid austral summer.

The ASP region is typically dominated by P. antarctica (Alderkamp et al., 2012; Yager et al., 2016) and is also connected to the Pine Island Glacier source of glacial meltwater. This is one of Antarctica's most rapidly melting glacial systems, from which meltwater flows westward and freshens the Ross Sea (Nakayama et al., 2014). Prior studies have demonstrated that glacial iron from the Amundsen Sea can support the massive $P$. antarctica blooms and high primary productivity in this region (Gerringa et al., 2012; Planquette et al., 2013; Sherrell et al., 2015). Observations of iron sources in the ASP include: intrusions of modified Circumpolar Deep Water (mCDW) onto the continental shelf (Sherrell et al., 2015), sediment resuspension (Planquette et al., 2013; Sherrell et al., 2018), ice shelves (Alderkamp et al., 2012; Gerringa et al., 2012), and sea ice (Lannuzel et al., 2016). For example, the Pine Island Glacier is measured to be the largest source of dissolved iron and total dissolvable iron to phytoplankton up to $150 \mathrm{~km}$ from the glacier (Gerringa et al., 2012). Finally, prior nutrient amendment experiments in the ASP have shown that the phytoplankton can be iron-replete (no iron-limitation), suggesting that at times there is sufficient bioavailable iron (Mills et al., 2012).

Pattern of seasonal succession A critical gap in our understanding of this region's microbial ecology is the lack of a temporally-resolved dataset of in situ (micro)nutrient concentrations and microbial community composition. A literature review of available cruise observations allows us to develop a model of seasonal succession in this region and the possible controlling mechanisms. The current paradigm of seasonal marine microbial succession in coastal Antarctic seas is as follows: In the early spring and summer there is sufficient dissolved iron for phytoplankton growth when $P$. antarctica 
dominates the algal community (Arrigo et al., 1999; Petrou et al., 2016; Sedwick et al., 2000; Smith et al., 2003), resulting in a buildup of dissolved organic carbon (DOC) that supports a bacterioplankton bloom (Ducklow et al., 2001), followed by lower iron concentrations in the later summer except near the more iron-enriched, strategies waters near the melting sea ice edge where diatoms blooms. This pattern is speculated to result from bottom-up controls of iron availability, irradiance, and vertical mixing (Sedwick et al., 2000). Some frameworks posit that the wintertime reserve of dissolved iron, combined with increasing meltwater supply, will support large phytoplankton at the start of the spring bloom and then transition to small phytoplankton types in the summer and autumn (Tagliabue et al., 2016). Pigment analyses have shown that after the spring bloom of $P$. antarctica, diatoms can reach bloom concentrations that are comparable or higher than $P$. antarctica blooms in the late spring and summer (Smith Jr et al., 2006, 2012). Although these observations are disparate, together they form a consistent pattern of seasonal succession. The driving mechanisms, however, are still being determined.

The dominant bloom-forming phytoplankton- $-P$. antarctica and diatoms - vastly differ in their cellular stoichiometry, with direct consequences on carbon sequestration, regional biogeochemistry via carbon and sulfur cycling, and ecosystem structure due to their different sizes and ecophysiologies (Arrigo et al., 1999). Polar phytoplankton can be large, with diatom radii on the order of $100 \mu \mathrm{m}$, some of which are chain-forming. Though individual $P$. antarctica cells range in radius from $3 \mu \mathrm{m}$ to $8 \mu \mathrm{m}$, colonies can grow to be on the order of $10-1000 \mu \mathrm{m}$ in radius, with some being several millimeter large (Schoemann et al., 2005). Millimeter-size colonies are often observed during $P$. antarctica blooms. They affect the size structure of the entire community, due to reduced grazing pressure and also bacterioplankton consuming the $P$. antarctica colonial matrix during senescence. $P$. antarctica is the dominant producer of DMSP, an organosulfur compound that is metabolized to DMS, a climatically active gas that induces cloud formation and has a key role in the sulfur biogeochemical cycle. Finally, $P$. antarctica and diatoms greatly differ in their stoichiometry of $\mathrm{C}: \mathrm{P}$ and $\mathrm{N}: \mathrm{P}$, and consequently their capacity to draw down both atmospheric carbon and nutrients (Arrigo et al., 2002; Dunbar et al., 2003). Both bloom-formers have non-Redfield ratios of $\mathrm{C}: \mathrm{N}: \mathrm{P}$, with $P$. antarctica having higher ratios and more potential carbon draw down. To illustrate, $P$. antarctica can remove roughly $100 \%$ more $\mathrm{CO}_{2}$ per mole of assimilated $\mathrm{PO}_{4}$ compared to diatoms, highlighting the higher carbon export potential of $P$. antarctica colonies (Arrigo et al., 2002). This is due to P. antarctica's colony matrix, which is made up of a carbohydrate-protein mix and increases the carbon quota for $P$. antarctica that can be rapidly exported to depth (DiTullio et al., 2000). Any environmental or ecosystem changes that favor the growth of diatoms over $P$. antarctica (or vice versa) have important consequences for the efficiency of the biological carbon pump and community dynamics that are utterly dependent on phytoplankton primary productivity. It is especially important to improve our understanding of the controls on phytoplankton dynamics in the time of increased anthropogenic climate change, when iron supplies from sea-ice and glacial melt are already changing rapidly. 

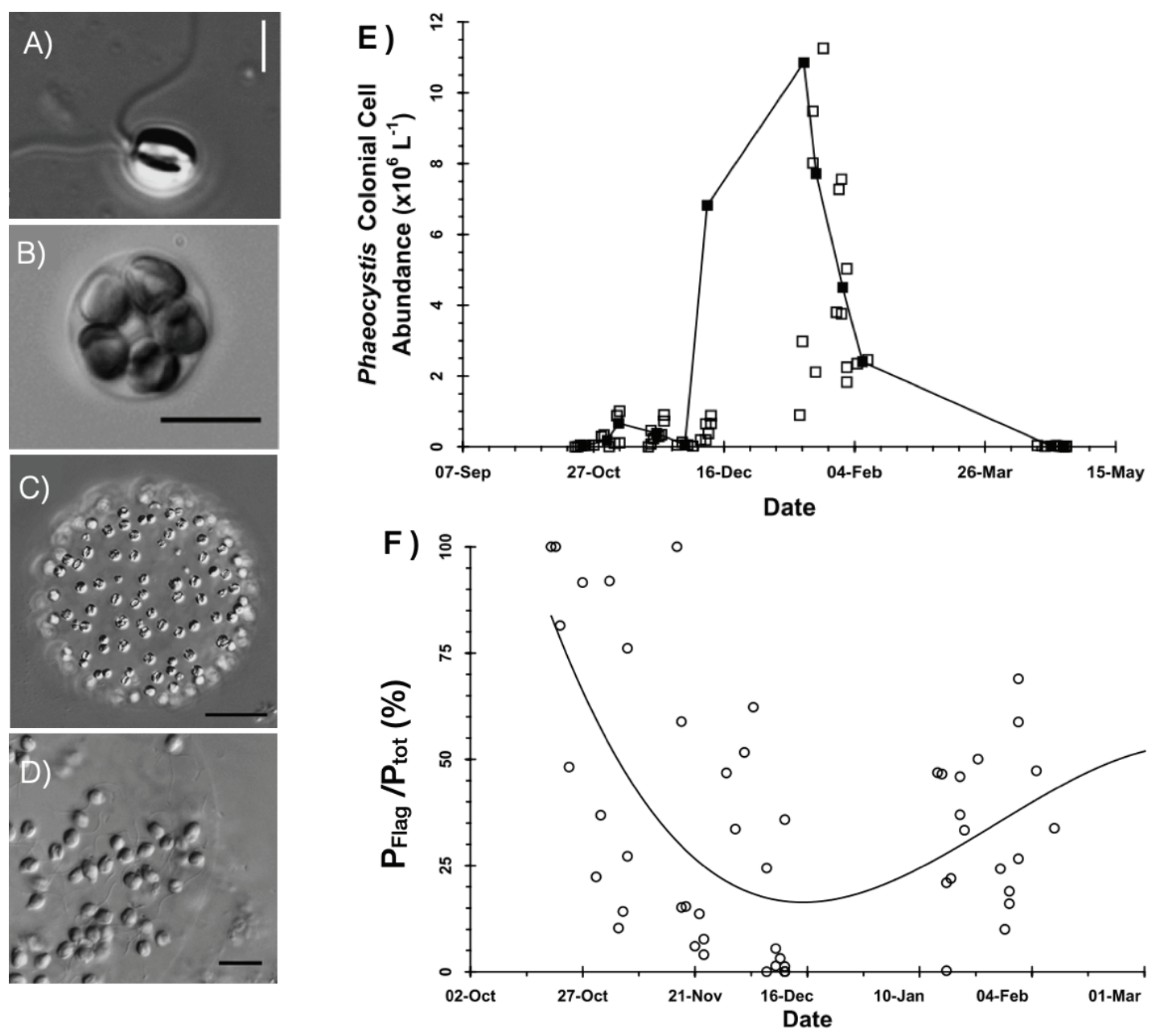

Figure 4-1: P. antarctica morphotypes have different ecophysiology and blooms are primarily a life cycle event, wherein colonies dominate the community at the peak of the austral spring-summer bloom. Images A-D are SEM photos of a subset of $P$. antarctica life cycle stages from (A) flagellated, motile unicells, to (B) small colonies of larger, non-motile cells, and $(\mathrm{C})$ mature, large colonies, and finally (D) a colony containing flagellates cells that will leave the colony structure. Figures E-F show a time series measurement of (E) P. antarctica colonial cell abundance and $(\mathrm{F})$ the ratio of flagellated, solitary cells to total cells. This image is a composite of results from Zingone et al. (2011) (A-D) and Smith et al. (2003) $(\mathrm{E}-\mathrm{F})$.

Further details on Phaeocystis antarctica $P$. antarctica is a keystone phytoplankton species in Antarctic systems due to blooms, which are primarily a life cycle event for this species (Schoemann et al., 2005; Verity et al., 2007). A brief discussion of the morphology and ecology of $P$. antarctica solitary cells colonies is needed for context for the model and to explain the succession of P. antarctica morphotypes. Interested readers are referred to an excellent review of Phaeocystis by Schoemann et al. (2005) and Rousseau et al. (2007) for more background on the global distribution of the genus, traits and ecology of its main species, and overview 
of its polymorphic life cycle.

$P$. antarctica has a complex life cycle, which is not fully understood. In the environment, it is most often observed in its two main morphotypes: flagellated solitary cells and spherical colonies composed of colonial cells, as seen in Fig.4-1 (though other stages like cysts and reproductive cells exist) (Rousseau et al., 1994). Phaeocystis colonies are a distinct life cycle stage with cells growing together in a mucilaginous matrix. $P$. antarctica can metamorphose between the smaller motile solitary cells and larger, non-motile colonial cells that live in a collective colony unit within a matrix. The colonies range in size from tens of microns to millimeters in diameter, with anywhere from two to thousands of colonial cells. Colonial cells produce polysaccharide exopolymers (with calcium ions and manganese ions) and glycoproteins that form a gel in seawater (Janse et al., 1996; Van Boekel, 1992). This mucilaginous matrix acts as a semipermeable "skin" that protects colonial cells, while the center of the colony is aqueous (mostly seawater and exudates of the cells) (Schoemann et al., 2001). P. antarctica dominates in the early spring blooms, under iron-replete conditions and lower irradiances compared to summer. This has been attributed to $P$. antarctica's neutral buoyancy of the colonies, and its maintaining near-maximal photosynthetic rates under low light (Arrigo et al., 1999), perhaps owing to the small size of single-cell $P$. antarctica. Recent lab culture experiments demonstrate that $P$. antarctica (strain CCMP 1871) is triggered into colony formation due to higher Fe concentrations, reported in Becquevort et al. (2007) and Bender et al. (2018) and the results in Chapter 2.

The colonial life stage of Phaeocystis likely serves several functions with a range of fitness advantages. The cells are located at the periphery of the colony matrix, about $15 \mu \mathrm{m}$ to $20 \mu \mathrm{m}$ below the matrix surface. As colony size increases, the number of cells per surface area decreases significantly, in other words individual cells are relatively more spread apart in the colony (Rousseau et al., 1994). This spatial arrangement of isometrically spread apart cells along the colony periphery protects individual cells from diffusion-limited nutrient acquisition and self-shading (Ploug et al., 1999). Large $P$. antarctica colonies are neutrally buoyant or sink at low velocities, since the majority of their volume is seawater in the center (Ploug et al., 1999; Van Boekel, 1992). The colony mucus is also a defense against viruses and other microzooplankton grazers (Nejstgaard et al., 2007). Studies vary in their reports of bacteria and protists colonizing $P$. antarctica colonies, including a dinoflagellates that lives off $P$. antarctica cells via kpletoplasty and bacteria that live on and within the colony itself. Some reports mention that colonies are only colonized during the senescent/stationary phase of blooms and is often reported to not be colonized during active growth (Becquevort et al., 1998; Davidson and Marchant, 1987; Putt et al., 1994); however, this is inconsistent with other anecdotal accounts of bacteria-rich colonies in the midst of a $P$. antarctica bloom. Finally, laboratory studies and field studies produce still debatable results on grazing pressure between morphotypes, but there is general agreement that the colony matrix is a deterrent to microzooplankton grazers. The colonies have a distinct micro-environment associated with photosynthesizing cells that have strong oxygen and $\mathrm{pH}$ gradients that can affect trace metal reactivity (Ploug et al., 1996, 1999; Schoemann et al., 2001). It has been demonstrated with radiotracer 
culture experiments that $P$. antarctica colonies can accumulate both $\mathrm{Mn}$ and $\mathrm{Fe}$ in the colony matrix, as a result of complexation, adsorption, oxidation, precipitation; and furthermore, assimilate these trace metals intracellularly (Schoemann et al., 2001). It has been speculated that this results in a significant "extracellular reservoir" of accumulated Mn, and to a lesser extent Fe. Even if assimilation accounts for only $10 \%$ of the total uptake of these trace metals into the colonial cells (as observed by Schoemann et al. (2001)), the competitive advantage of storing Fe in the colony matrix has a potentially huge effect on microbial community composition and dynamics.

What drives the seasonal succession these ecosystems? The existing paradigm for the spring blooms of $P$. antarctica and summer blooms of diatoms is based on bottom-up controls, but recent studies challenge this paradigm and suggest that other micronutrients may play a key role in driving observed community dynamics. Arrigo et al. (1999) and Arrigo and van Dijken (2004) proposed that irradiance, Fe supply, and mixed layer depth controls phytoplankton bloom dynamics in this region, wherein $P$. antarctica thrives in weakly-stratified waters, while diatoms dominate in stratified waters. However, $P$. antarctica also forms massive blooms in areas with deep and shallow mixed layer depths (southern Ross Sea and Amundsen Sea) in the dim light of spring and constant sun of summer (Delmont et al., 2014; Williams et al., 2016). Furthermore, Sedwick et al. (2007) nicely reviews several studies that demonstrate $P$. antarctica's ability to acclimate to a wide range of light conditions, predominantly by modulating the balance of its photosynthetic/accessory pigments (DiTullio et al., 2000). Some of these studies suggest that the irradiance characteristics of diatoms and colonial P. antarctica are similar (van Hilst and Smith Jr, 2002). Although irradiance and mixed layer depth have been invoked to describe the seasonal succession of both $P$. antarctica morphotypes and diatoms, studies find evidence to support and refute this hypothesis, making it inconclusive (Arrigo et al., 1998a; Boyd, 2002; Petrou et al., 2016; Smith et al., 2003; Tagliabue and Arrigo, 2005).

Instead, a combination of micronutrient limitation and grazing may better explain the spatial-temporal distributions of the two dominant phytoplankton groups in this region and also the observed bacterioplankton bloom after $P$. antarctica. Specifically, Fe is known to limit $P$. antarctica growth when ambient concentrations are low and affect its transition from single cells to colonies (Bender et al., 2018; Sedwick et al., 2000). Grazing studies also agree that the colony matrix/mucus is a deterrent for some grazers and field observations of blooming colonies show minimal grazing during growth (Ducklow et al., 2006; Nejstgaard et al., 2007). Field and laboratory experiments both confirm that $\mathrm{B}_{12}$ can have an affect on primary production and community composition. A combination of iron and $\mathrm{B}_{12}$ limitation has been proposed to drive the seasonal pattern of $P$. antarctica to bacteria to diatoms (Bertrand et al., 2012). We expand on this idea through the observations in the lab and field chapters of this thesis, which showed that $P$. antarctica (strain CCMP 1871) is not dependent on $\mathrm{B}_{12}$ (but can use it if available) and that diatoms are strongly correlated to both cobalt and $\mathrm{B}_{12}$ uptake across a wide swath of Antarctic surface waters from the Amundsen to the Ross Sea. Now, we continue to explore the potential for an Fe-B 12 limited system 
through the development of this computational model.

\subsubsection{Micronutrient-limited growth in Antarctic marine microbial communities}

In the high-nutrient, low-chlorophyll (HNLC) waters of the Southern Ocean, the marine phytoplankton are typically primarily limited by iron $(\mathrm{Fe})$ and co-limited or secondarily-limited by cobalamin (vitamin $\mathrm{B}_{12}$ ) (Bertrand et al., 2007; Moore et al., 2013; Sedwick et al., 2000). Through shipboard nutrient-amendment experiments, $P$. antarctica has only been shown to be Fe-limited; while diatoms have been shown to be Fe-limited and co-limited by Fe and $\mathrm{B}_{12}$ (Bertrand et al., 2007, 2011b; Coale et al., 2003; Sedwick et al., 2000). In one study, Fe addition resulted in P. antarctica increasing in relative abundance from $<5 \%$ to $20 \%$ of the phytoplankton community (Coale et al., 2003). P. antarctica dominates the phytoplankton community in the southern coastal region of the Ross Ice Shelf and along the coast of the Amundsen Sea, close to glacial shelf water input. In another study Fe addition increased $P$. antarctica abundance, but a combination of $\mathrm{Fe}$ and $\mathrm{B}_{12}$ additions increased overall phytoplankton growth, especially of Pseudonitzchia subcurvata (Bertrand et al., 2007), which was later found to be a $\mathrm{B}_{12}$-dependent (Ellis et al., 2017). This same study also found that sites with low bacterial abundances were stimulated by $\mathrm{B}_{12}$ additions, solidifying that bacterioplankton production is an important factor influencing phytoplankton community composition (Bertrand et al., 2007). In this region, heterotrophic bacteria are primarily limited by organic carbon, due to seasonally low primary productivity, and can be secondarily limited by iron (Bertrand et al., 2011b; Church et al., 2000; Kirchman et al., 2000).

In general, both dissolved $\mathrm{Fe}$ and $\mathrm{B}_{12}$ are scarce in Antarctic surface waters compared to other oceanographic regions, increasing the potential for micronutrient limitation. Fe is generally lower due to decreased inputs from land and aeolian sources. $\mathrm{B}_{12}$ is low due to reduced bacterial production, no marine cyanobacteria, and higher UV-degradation, despite a generally high reservoir of cobalt needed to synthesize the vitamin. Field observations report a range of dissolved iron from $<0.1$ to $0.5 \mathrm{nM}$ between the spring and late summer (Coale et al., 2005; Fitzwater et al., 2000; Sedwick and DiTullio, 1997; Sedwick et al., 2000). Based on shipboard ironamendment experiments, $P$. antarctica is Fe-limited at $0.22 \mathrm{nM}$ but not at $0.38 \mathrm{nM}$ ambient concentrations (Sedwick et al., 2007). $\mathrm{B}_{12}$ is very scarce, on the order of $<0.1$ to 1 pM (Panzeca et al., 2009).

\subsubsection{Conceptual Model}

First, we ask: What drives bloom formation and seasonal succession in coastal Antarctic marine microbial communities?

We propose an alternative hypothesis for the seasonal succession of $P$. antarctica morphotypes to diatoms based on micronutrient limitation, summarized in Fig.4-2. The conceptual model is as follows. With the onset of spring, low irradiances enable photosynthesis that is supported by a winter reserve of iron and input of dissolved 
Fe from sea ice and glacial melt. At these initial low light and Fe conditions, $P$. antarctica solitary cells are able to grow due to their small size. At a sufficient iron concentration, the solitary cells metamorphose into colonial cells, which are non-motile and larger in size, and start to form colonies. These larger colonial cells living within the colony also require more $\mathrm{Fe}$, but have the benefit of very low grazing rates due to their mucilaginous exterior. The biomass of $P$. antarctica accumulates in the ecosystem and dead cells contribute to the detritus and labile dissolved organic carbon pool. This increase in dissolved organic carbon enables the growth of heterotrophic bacteria, which bloom after $P$. antarctica. Some portion of the growing bacterial population synthesizes $B_{12}$, which can be either excreted or released from lysed cells. This renewed supply of $\mathrm{B}_{12}$ enables the growth of $\mathrm{B}_{12}$-auxotrophic diatoms, which respond quickly due to their relatively high growth rates and increased $\mathrm{B}_{12}$ transporter (CBA1) expression under limited conditions (Bertrand et al., 2011a). Depending on community activity, diatoms can be either primarily limited by $\mathrm{Fe}$ or $\mathrm{B}_{12}$, which determines the timing of diatom blooms.

\subsection{Methods: Model Development}

Here, we present a model ecosystem consisting of $P$. antarctica solitary cells and colonial cells in fixed-size colony, diatoms, and heterotrophic bacteria to examine seasonal succession in Antarctic coastal ecosystems. The model framework is presented in Fig.42 (described in the figure caption), and the model equations are in the following section. We assume that light and macronutrients are abundant and that iron is the primary limiting nutrient, as would be expected in the high-nutrient, low-chlorophyll (HNLC) Southern Ocean. The model is first explored with just $P$. antarctica morphotypes under iron limitation to examine the dynamics of a population composed of two interacting morphotypes. Field and lab observations agree that $P$. antarctica can be Fe-limited in situ and that iron concentrations can affect morphotype distributions (Bender et al., 2018; Bertrand et al., 2007; Sedwick et al., 2000). Accordingly, we model the two dominant morphotypes of $P$. antarctica with an Fe-dependent transition rate. Given sufficient iron, solitary cell biomass will transition into colonial biomass at an iron-dependent rate $T_{\mathrm{ic}}$, and colonial biomass returns into solitary cells at a rate $T_{\mathrm{ci}}$ that is a fixed fraction transition rate of solitary to colonial cells, which we take to be $10 \%$. To account for multiple cells living inside of a colony matrix, we use a diffusive boundary layer (DBL) model to represent the resource concentration $C_{0}$ that individual cells would experience due to the size of the entire colony (Ploug et al., 1999). The DBL is modeled for solitary cells, colonial cells, and diatoms. In this model, diatoms are $\mathrm{B}_{12}$-auxotrophs and can be limited by either $\mathrm{Fe}$ or $\mathrm{B}_{12}$, following Liebig's Law of the Minimum. The dead cells of the phytoplankton and bacteria contribute to the pool of labile/semi-labile dissolved organic matter (DOC), which is the limiting resource for heterotrophic bacteria. As bacteria grow, a fraction of the community is able to synthesize $\mathrm{B}_{12}$ at a constant rate (Rodionov et al., 2003). Thus, as bacteria grow $\mathrm{B}_{12}$ availability increases, which can then be acquired by diatoms. Using this simplified set of microbial functional types, we examine the ecosystem at 


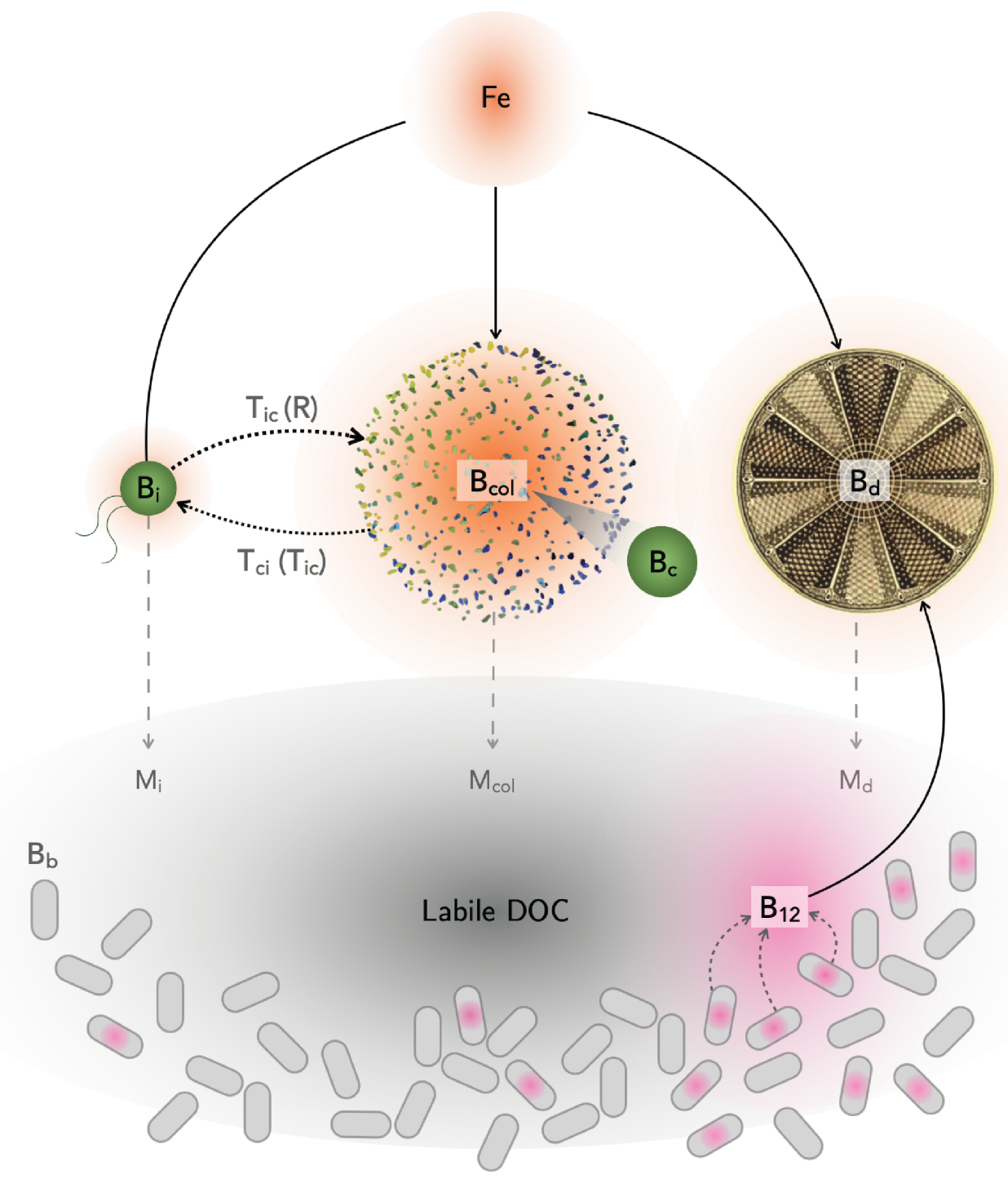

Figure 4-2: Model framework: P. antarctica solitary cells, colonial cells, and diatoms all compete for iron $(\mathrm{Fe})$, represented by resource concentration $R$. In the model, we characterize these populations in terms of carbon biomass $B_{\mathrm{i}}, B_{\mathrm{col}}$, and $B_{\mathrm{d}}$, of the $P$. antarctica individuals, colonies, and diatoms respectively. P. antarctica colonies are composed of colonial cells, with biomass $B_{\mathrm{c}}$, which are larger than solitary cells and have a higher iron half-saturation constant $K_{R}$. With sufficient iron, solitary cells transition into colonial cells at a rate $T_{\text {ic }}$ that depends on iron concentration; colonial cells transition back into solitary cells at a smaller relative rate $T_{\mathrm{ci}}\left(T_{\mathrm{ic}}\right)$. The sum of all phytoplankton and bacterioplankton mortality contributes to a pool of detritus with a concentration of labile dissolved organic carbon DOC that is the food source for heterotrophic bacteria, with biomass $B_{\mathrm{b}}$. A fraction of the heterotrophic bacterial community synthesizes vitamin $B_{12}$, which is released into ambient seawater. The diatoms in this model are $\mathrm{B}_{12}$-auxotrophs, and are dependent on the overall ecosystem productivity to fuel bacterial growth and $\mathrm{B}_{12}$ production.

steady state and a seasonally-forced model (with a sinusoidal iron supply) to examine seasonal succession. 


\subsubsection{Allometric scaling of traits}

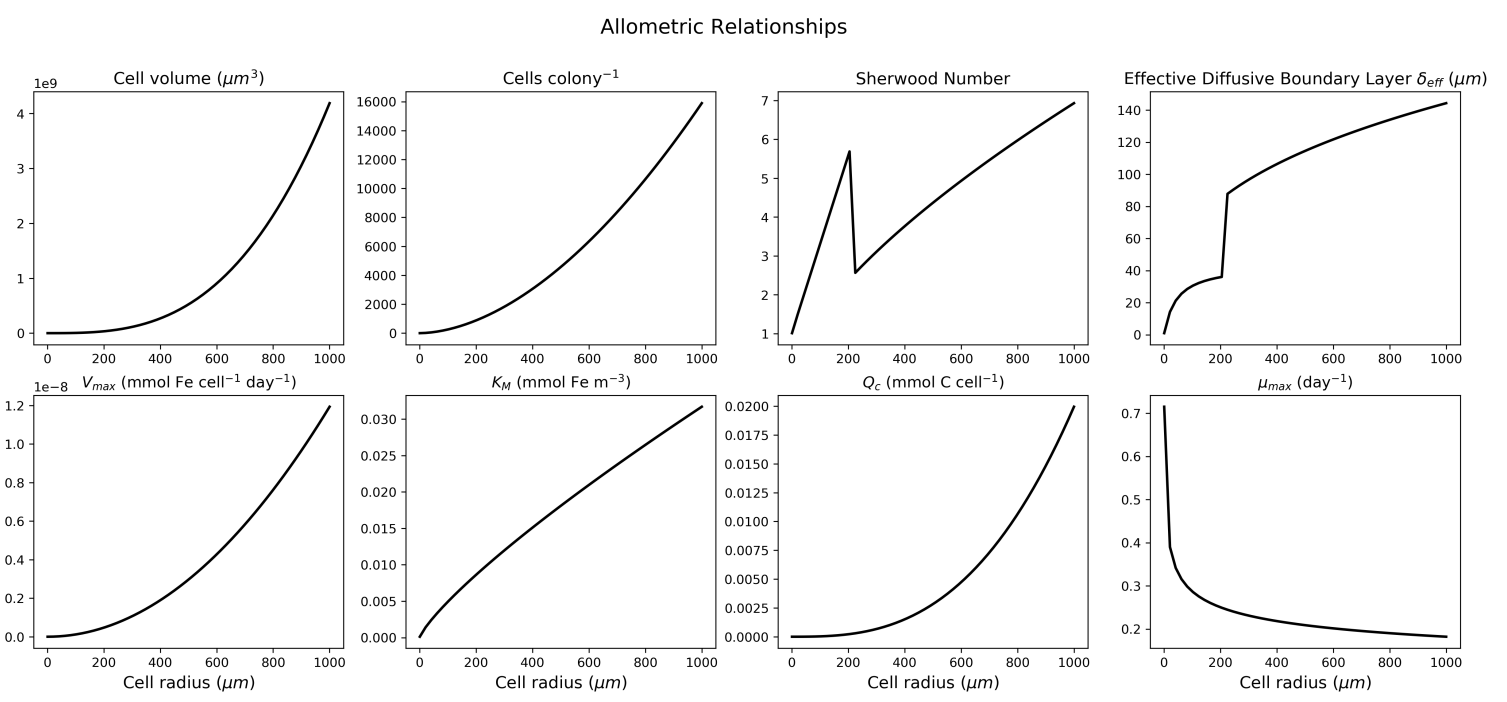

Figure 4-3: Allometric scaling relationships applied in the model. There are discontinuities in the scaling relationships of cell volume with Sherwood Number and the effective diffusive boundary layer due to piece-wise calculations in different Renyolds number and Peclet number regimes (Ploug et al., 1999).

Phytoplankton functional types are modeled with size-scaled traits for several parameters. In phytoplankton communities, size structure is an important factor that influences food-web dynamics (especially grazing pressure), biogeochemical cycling, and carbon-climate feedbacks (Finkel et al., 2010; Marañón, 2015). Following allometric theory, a diverse set of phytoplankton traits scale with body size with empiricallyderived relationships of the form $T=a v^{b}$, where $v$ is cell volume, $T$ is a specific trait (e.g., cells colony ${ }^{-1}$, maximum resource uptake rate $V_{\max }$, half-saturation constant $K_{R}$, and $a$ and $b$ are constants. The exponent $b$ is relatively constant for a range of phytoplankton types, but the factor $a$ varies by broad phytoplankton groups (Litchman et al., 2007; Ward et al., 2012). The following traits are size-scaled: the maximum nutrient uptake rate $V_{\max }$, nutrient half-saturation constant $K_{R}$, cellular carbon quota $Q_{\mathrm{C}}$, colonial cell count (cells colony ${ }^{-1}$ ), as seen in Fig.4-3. Maximum growth rates $\mu_{\max }$ and mortality rates $m$ are assigned to account for the high growth rates of diatoms and low mortality of colonies, which diverge from typical scaling rules.

In general, growth rate decreases with cell size (Tang et al., 2010); however, there are always exceptions to the rule. Notably, some large diatoms can have comparatively high growth rates (Dutkiewicz et al., 2020; Verdy et al., 2009). Larger colonial cells of both Phaeocystis globosa and Phaeocystis pouchetii have higher growth rates than single cells when grown under identical conditions, as measured using DNA-cell-cycle methods, with gross growth rates being 1.5 to 3.8 times greater than solitary cells (Veldhuis et al., 2005). We observe higher colonial cell growth rates compared to solitary cells in nutrient-replete conditions $\left(+\mathrm{Fe}\right.$ and $+\mathrm{B}_{12}$ in the lab study presented in Chapter 2, Fig.2-2). It is important to note that maximum growth rates of polar 
phytoplankton tend to be lower than their temperate-water counterparts due to slowed metabolisms in cooler temperatures.

Nutrient uptake with a diffusive boundary layer: To account for colony nutrient uptake as a function of the collective activity of all colonial cells, a modified Michaelis-Mention equation was used to describe the maximum nutrient uptake rate $V_{\max }$, which is equal to the area-integrated diffusive flux of iron $Q\left(\mu \mathrm{mol} \mathrm{Fe} \mathrm{cell}{ }^{-1} \mathrm{~d}^{-1}\right)$ through the colony's diffusive boundary layer, in a turbulent environment with shear (Ploug et al., 1999):

$$
\operatorname{Sh} 4 \pi r_{0} D\left(R-C_{0}\right)=\frac{n_{\mathrm{col}} V_{\mathrm{max}} C_{0}}{K_{R}+C_{0}}
$$

The left-hand side of the equation is $Q$, with the Sherwood number Sh the ratio of convective to diffusive mass transport), colony radius $r_{0}$, the resource diffusion coefficient $D$, the bulk water resource concentration $R$, and the resource concentration $C_{0}$ at the colony surface or cell surface. The right-hand side of the equation is the uptake rate $V$, where $n_{\text {col }}$ is the number of colonial cells, $V_{\max }$ is the maximum uptake rate per colonial cell, and $K_{R}$ is the half-saturation constant for a cell (solitary, colonial, or diatom), following Ploug et al. (1999). If this model is applied to a single cell, then $n_{\text {col }}=1$ for solitary cells and diatoms. In this model, $P$. antarctica cells within a colony have traits $\left(K_{R}, V_{\max }\right.$, and $\left.Q_{\mathrm{C}}\right)$ that are scaled according to their cell size $\left(r_{0}=3 \mu \mathrm{m}\right)$, not the colony size $\left(r_{0}=25 \mu \mathrm{m}\right)$. The important exception is that colonial cells experience the resource concentration $C_{0}$ at the colony boundary, following the equations in Fig.4-4.

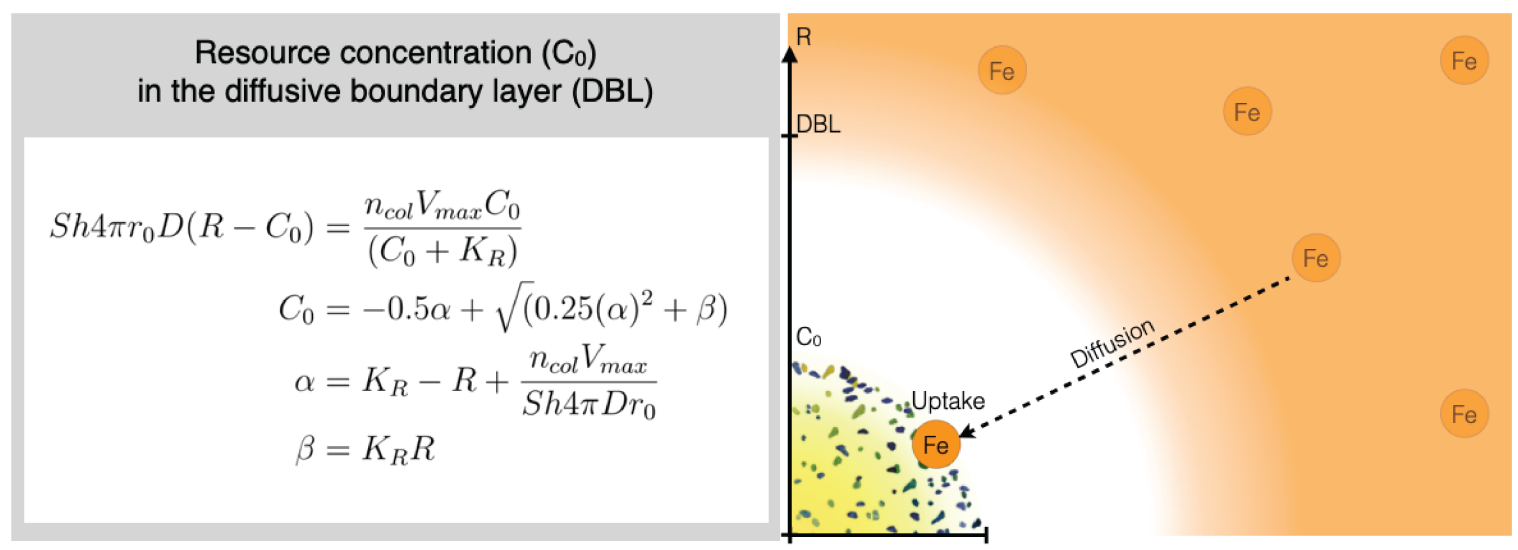

Figure 4-4: Resource concentration in the diffusive boundary layer (DBL). The resource $R$ in this model is iron $(\mathrm{Fe})$, and the concentration at the DBL is $C_{0}$.

The effective diffusive boundary layer thickness $\delta_{\text {eff }}$ is determined by the Sherwood number and radius: $\delta_{\text {eff }}=r_{0} /$ Sh. In this model, a colony $\left(r_{0}=25 \mu \mathrm{m}\right)$ at a fixed shear rate $\left(E=0.1 \mathrm{~s}^{-1}\right)$ has a $\delta_{\text {eff }}$ of $15.8 \mu \mathrm{m}$, while a solitary cell $\left(r_{0}=1.5 \mu \mathrm{m}\right)$ has a $\delta_{\text {eff }}$ of $1.47 \mu \mathrm{m}$. Due to the DBL of the colony, the colonial cells experience a significantly different resource concentration $C_{0}$ compared to ambient concentration 
of iron $R$, especially at low nutrient concentrations (Ploug et al., 1999). Specifically, compared to a free living cell of the same size, a colonial cell living inside the colony would experience a lower resource concentration, due to the thickness of the DBL and competition from other colonial cells. Solitary cells are always more competitive for low-concentration resources compared to the colonial cell, but as discussed the colony offers other advantages. At high shear rates, it is possible for a larger colony to have a reduced DBL and slightly increased diffusive flux into the colony matrix; although colonial cells are still at a disadvantage. For a more in-depth discussion on how to evaluate the Sherwood number, the reader is encouraged to review Ploug et al. (1999).

In this model, the resource is specifically dissolved iron $\left(\mathrm{Fe}_{\text {diss }}\right)$, which is the sum of all available iron species in seawater, with a diffusion coefficient of $D=$ $0.5 \times 10^{-6} \mathrm{~cm}^{2} \mathrm{~s}^{-1}$. This approach was taken because of the unknowns regarding which form(s) of iron are used by $P$. antarctica and diatoms (Timmermans et al., 2001a). The size-scaled half-saturation constants for $\mathrm{Fe}_{\text {diss }}\left(K_{R}\right)$ for P. antarctica is $0.16 \mathrm{nM}$ for solitary cells and $0.29 \mathrm{nM}$ for colonial cells, within range of measured values in laboratory experiments (Garcia et al., 2009; Oliver et al., 2019). Measured iron half-saturation constants for $P$. antarctica growth are $0.005 \mathrm{nM}$ to $0.042 \mathrm{nM}$ in (most likely) solitary cells via shipboard iron-amendment experiments (Coale et al., 2003 ), and $0.45 \mathrm{nM}$ in colonies via laboratory dose-response experiments grown under relatively low irradiance $\left(20 \mu \mathrm{E} \mathrm{m}^{-2} \mathrm{~s}^{-1}\right)$, though this experiment was done without EDTA to buffer the iron concentration (Sedwick et al., 2007). It has been suggested that the $K_{R}$ for $P$. antarctica for $\mathrm{Fe}_{\text {diss }}$ is on the order of $0.1 \mathrm{nM}$, with colonies requiring more iron, on par with values measured for some Southern Ocean diatoms (Sedwick et al., 2007; Timmermans et al., 2001a, 2004). However, based on allometric scaling,

the $K_{R, \mathrm{~d}}$ used for the model diatom is $1.6 \mathrm{nM}$, an order of magnitude higher, within a biologically reasonable range for Southern Ocean diatoms $0.19 \mathrm{nM}$ to $1.14 \mathrm{nM}$ grown under $60 \mu \mathrm{E} \mathrm{m}^{-2} \mathrm{~s}^{-1}$ irradiance (Timmermans et al., 2004).

\subsubsection{Model Formulation}

Micronutrient-driven community dynamics are explored in the ecosystem model is defined by the following set of equations for microbial functional type biomass $(B)$ and concentrations of inorganic $\left(\mathrm{Fe}_{\text {diss }}\right)$ and organic resources (DOC and $\mathrm{B}_{12}$ ). The model is based on Michaelis-Menten kinetics and uses linear mortality rates. The variables and parameters are documented in Tables 4.1 and 4.2. 


\begin{tabular}{|c|c|c|}
\hline Variable & Definition & Units \\
\hline$B_{\mathrm{i}}$ & Biomass solitary cells (i) & $\mathrm{mmol} \mathrm{C} \mathrm{m}{ }^{-3}$ \\
\hline$B_{\mathrm{c}}$ & Biomass colonial cells (c) & $\mathrm{mmol} \mathrm{C} \mathrm{m}{ }^{-3}$ \\
\hline$B_{\mathrm{col}}$ & Biomass colony (col) & $\mathrm{mmol} \mathrm{C} \mathrm{m}{ }^{-3}$ \\
\hline$B_{\mathrm{d}}$ & Biomass diatom cells $(\mathrm{d})$ & $\mathrm{mmol} \mathrm{C} \mathrm{m} \mathrm{m}^{-3}$ \\
\hline$B_{\mathrm{b}}$ & Biomass bacterial cells (b) & $\mathrm{mmol} \mathrm{C} \mathrm{m}{ }^{-3}$ \\
\hline$R$ & Concentration of iron $(\mathrm{Fe})$ & $\mathrm{mmol} \mathrm{Fe} \mathrm{m}^{-3}$ \\
\hline$C_{0}$ & Concentration of iron in the diffusive boundary layer & $\mathrm{mmol} \mathrm{Fe} \mathrm{m}{ }^{-3}$ \\
\hline $\mathrm{B}_{12}$ & Concentration of cobalamin (vitamin $\mathrm{B}_{12}$ ) & $\operatorname{pmol~B}_{12} \mathrm{~m}^{-3}$ \\
\hline DOC & Concentration of labile dissolved organic carbon (DOC) & $\mathrm{mmol} \mathrm{C} \mathrm{m}{ }^{-3}$ \\
\hline Parameter & Definition & Units \\
\hline$r_{0}$ & Cell radius $(\mathrm{i}, \mathrm{c}, \mathrm{col}, \mathrm{d}, \mathrm{b})$ & $\mu \mathrm{m}$ \\
\hline$v$ & Cell volume (i, c, col, d, b) & $\mu \mathrm{m}^{3}$ \\
\hline$\mu_{\max }$ & Maximum growth rate & $\mathrm{d}^{-1}$ \\
\hline$m$ & Mortality rate (via grazing and other processes) & $\mathrm{d}^{-1}$ \\
\hline$n_{\text {col }}$ & Number of cells per colony & cells colony $^{-1}$ \\
\hline$V_{\max }^{*}$ & Maximum nutrient uptake rate & umol Fe cell ${ }^{-1} \mathrm{~d}^{-1}$ \\
\hline$K_{R}$ & Half-saturation constant for Fe for growth, scaled from $\mathrm{N}$ & $\mu \mathrm{molN}$ \\
\hline$K_{\mathrm{DOC}}$ & Half-saturation constant $\mathrm{DOC}_{\text {labile }}$ for uptake & $\mathrm{mmol} \mathrm{C} \mathrm{m}{ }^{-3}$ \\
\hline$K_{\mathrm{B}_{12}}{ }^{* *}$ & Half-saturation constant $\mathrm{B}_{12}$ for growth & $\mathrm{nmol} \mathrm{m}^{-3}$ \\
\hline$S^{-12}$ & Supply rate of dissolved iron & $\mu \mathrm{mol} \mathrm{Fe} \mathrm{m}^{-3} \mathrm{~s}^{-1}$ \\
\hline$T_{\text {ic,max }}$ & Maxmimum transition rate of solitary cells to colonial cells & $d^{-1}$ \\
\hline$T_{\mathrm{ci}}$ & Transition rate of colonial cells to solitary cells & $\mathrm{d}^{-1}$ \\
\hline$R_{\text {crit }}$ & Critical iron concentration for morphotype transition & $\mu \mathrm{mol} \mathrm{Fe} \mathrm{m}^{-3}$ \\
\hline$w$ & Width of tanh function describing Fe-dependent morphotype transition rate & \\
\hline$Q_{\mathrm{C}}$ & Cellular carbon quota & mmol C cell ${ }^{-1}$ \\
\hline$Q_{\mathrm{B}_{12}}{ }^{* * *}$ & Cellular $\mathrm{B}_{12}$ quota & pmol B $_{12}$ cell $^{-1}$ \\
\hline$R_{\mathrm{C}: \mathrm{Fe}}$ & Redfield ratio for C:Fe & $\mu \mathrm{mol} \mathrm{C} \mu \mathrm{mol}^{-1} \mathrm{Fe}$ \\
\hline$S_{\mathrm{B}_{12}}^{\dagger}$ & Production/exudation rate of $\mathrm{B}_{12}$ & pmol cell ${ }^{-1} \mathrm{~d}^{-1}$ \\
\hline$y_{\mathrm{b}}$ & Biomass yield for bacteria & $\mathrm{mmol} \mathrm{C} \mathrm{mmol}^{-1} \mathrm{DOC}$ \\
\hline$V_{\max , \mathrm{DOC}}$ & Maximum specific resource uptake rate & $\mathrm{mmol}$ DOC $\mathrm{mmol}^{-1} \mathrm{C} \mathrm{d}^{-1}$ \\
\hline$D^{\ddagger}$ & Diffusion coefficient of dissolved iron $\left(\mathrm{Fe}_{\text {diss }}\right)$ & $\mathrm{cm}^{2} \mathrm{~s}^{-1}$ \\
\hline$E$ & Shear rate & $\mathrm{s}^{-1}$ \\
\hline Sh & Sherwood number $\mathrm{B}_{12}$ & \\
\hline$F_{\mathrm{DOC}}$ & Fraction of detritus that becomes labile DOC & \\
\hline$F_{\mathrm{B}_{12}}$ & Fraction of bacterial community that synthesizes $B_{12}$ & \\
\hline
\end{tabular}

Table 4.1: Model variables and parameters with units. For specific values and allometric scaling relationships, see Table 4.2.

${ }^{*} V_{\text {max }}$ was converted from nmol $\mathrm{N}_{\text {cell }}{ }^{-1} \mathrm{~d}^{-1}$ from Ho et al. (2003); Litchman et al. (2007); Verdy et al. (2009).

${ }^{* *} K_{\mathrm{B}_{12}}$ for growth range from $<0.1$ to $10 \mathrm{pM}$ (Droop, 2007; Tang et al., 2010; Taylor and Sullivan, 2008).

${ }^{* * *} Q_{\mathrm{B}_{12}}$ is from Bertrand et al. (2013); Tang et al. (2010).

$\dagger S_{\mathrm{B}_{12}}$ was estimated based on data from Croft et al. (2005) and Table 1 from Bonnet et al. (2010) and Taylor and McLennan (1985).

${ }^{\ddagger} D$ is derived from Völker and Wolf-Gladrow (1999); Yuan-Hui and Gregory (1974) and extrapolated to $0^{\circ} \mathrm{C}$, and converted from $\mathrm{dm}^{2} \mathrm{~s}^{-1}$ (Timmermans et al., 2001a). 


\section{Model equations}

$$
\begin{aligned}
& \underset{\substack{\text { solitary } \\
\text { cells }}}{\frac{d B_{\mathrm{i}}}{d t}}=\mu_{\text {max }, \mathrm{i}} \frac{C_{0, i}}{C_{0, i}+K_{R, i}} B_{\mathrm{i}}-\underset{\text { growth }}{C_{\mathrm{i}} B_{\mathrm{i}}}-\underset{\substack{\mathrm{ic} \\
\text { transition } \\
\mathrm{i} \rightarrow \mathrm{c}}}{T_{\mathrm{i}}(R) B_{\mathrm{i}}}+\underset{\substack{\text { transition } \\
\mathrm{c} \rightarrow \mathrm{i}}}{T_{\mathrm{c}} B_{\mathrm{c}}}
\end{aligned}
$$

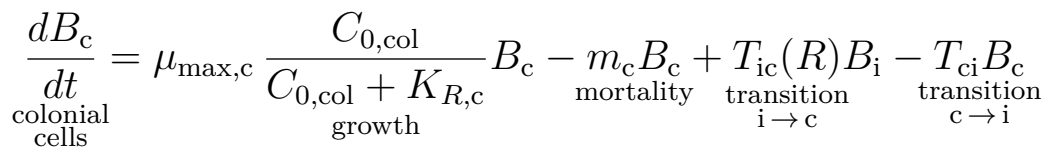

$$
\begin{aligned}
& \frac{d B_{\mathrm{d}}}{d t}=\mu_{\text {max }, \mathrm{d}} \lim _{\text {growth }} B_{\mathrm{d}}-\underset{\text { mortality }}{m_{\mathrm{d}} B_{\mathrm{d}}} \\
& \text { where } \lim _{\mathrm{d}}=\min \left[\frac{C_{0, \mathrm{~d}}}{C_{0, \mathrm{~d}}+K_{R, \mathrm{~d}}}, \frac{\mathrm{B}_{12}}{\mathrm{~B}_{12}+K_{\mathrm{B}_{12}, \mathrm{~d}}}\right] \\
& \frac{d B_{\mathrm{b}}}{d t}=y_{\mathrm{b}} V_{\text {max }, \mathrm{DOC}} \frac{\mathrm{DOC}}{\text { bacteria }} \underset{\text { growth }}{\mathrm{DOC}+K_{\mathrm{DOC}}} B_{\mathrm{b}}-\underset{\text { mortality }}{m_{\mathrm{b}} B_{\mathrm{b}}} \\
& \frac{d R}{d t}=\underset{\substack{\text { external } \\
\text { resource } \\
\text { supply }}}{S}-\mu_{\max , \mathrm{i}} \frac{C_{0, \mathrm{i}}}{\substack{\left(C_{0, \mathrm{i}}+K_{R, \mathrm{i}}\right) \\
\text { solitary uptake }}} \frac{B_{\mathrm{i}}}{R_{\mathrm{C}: \mathrm{Fe}}} \\
& -\mu_{\text {max }, \mathrm{c}} \frac{C_{0, \mathrm{col}}}{\begin{array}{c}
\left(C_{0, \mathrm{col}}+K_{R, \mathrm{c}}\right) \\
\text { colonial uptake }
\end{array}} \frac{B_{\mathrm{c}}}{R_{\mathrm{C}: \mathrm{Fe}}}-\mu_{\text {max }, \mathrm{d}} \lim _{\mathrm{d}} \frac{B_{\mathrm{d}}}{R_{\mathrm{C}: \mathrm{Fe}}} \\
& \underset{\text { labile DOC }}{\frac{d \mathrm{DOC}}{d t}}=F_{\mathrm{DOC}}\left(m_{\mathrm{i}} B_{\mathrm{i}}+m_{\text {col }} B_{\text {col }}+m_{\mathrm{d}} B_{\mathrm{d}}+m_{\mathrm{b}} B_{\mathrm{b}}\right) \\
& -V_{\max , \mathrm{DOC}} \frac{\mathrm{DOC}}{\underset{\text { bacteria uptake }}{\mathrm{DOC}}+K_{\mathrm{DOC}}} B_{\mathrm{b}}
\end{aligned}
$$

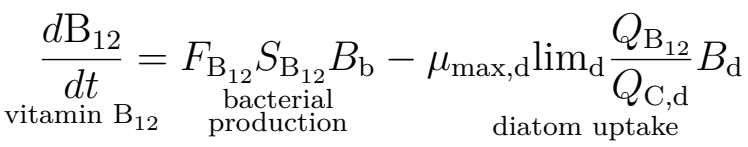

The limiting resource $R$ is dissolved iron with an external supply rate $S$. It is consumed by $P$. antarctica solitary cells, colonial cells, and diatoms, according to an extended Redfield ratio for iron Eq. (4.7) (Ho et al., 2003). P. antarctica growth-Eqs. (4.2) and (4.3) - is only limited by iron availability, which consequently affects its transition in and out of colonies. Diatoms require both $\mathrm{Fe}$ and $\mathrm{B}_{12}$, and their growth rate depends on the balance between resource concentration and halfsaturation constants via a Michaelis-Menten relationship and Leibig's Law of the Minimum: Eqs. (4.4) and (4.5) respectively. Bacterial growth, Eq. (4.6), depends on the availability of dissolved organic carbon (DOC), which, per Eq. (4.8), comes from a fraction $F_{\mathrm{DOC}}$ of the dead biomass from all groups. Both $P$. antarctica and diatoms have a diffusive boundary layer that affects their resource availability for iron from $R$ to $C_{0}$. Bacteria have an assimilation efficiency $y_{\mathrm{b}}$ to convert DOC into biomass - the rest is used in respiration - so bacterial growth is governed by Eq. (4.6). 
Finally, Eq. (4.9) models that $\mathrm{B}_{12}$ is only synthesized by a subset $F_{\mathrm{B}_{12}} \approx 30 \%$ of the bacteria (Raux et al., 2000) at a fixed excretion rate per cell $S_{\mathrm{B}_{12}}$ (Taylor and Sullivan, $2008)$, and is consumed only by diatoms at a fixed cellular quota of C:B $\mathrm{B}_{12}\left(Q_{\mathrm{C}, \mathrm{d}} / Q_{\mathrm{B}_{12}}\right)$ (Tang et al., 2010). The subscripts i, c, and col refer to $P$. antarctica solitary cells, colonial cells, and colonies, and the subscripts $\mathrm{d}$ and $\mathrm{b}$ refer to diatoms and bacteria.

$P$. antarctica life cycle transitions: In this model, $P$. antarctica transitions between solitary and colonial cells. The transition rate $T_{\text {ic }}$ is modeled as a saturating function of the iron concentration $R$, with a small transition width and a critical iron concentration $R_{\text {crit }}$ that sets when solitary cells will transition (Fig.4-5). The critical iron concentration is based on a dose-response study of $P$. antarctica, for which higher iron concentrations resulted in the formation of colonies (Bender et al., 2018). For this analysis, $R_{\text {crit }}$ was chosen as $0.1 \mathrm{nM}$, and in the aforementioned lab study colony formation began within the range of $0.12 \mathrm{pM}$ to $0.74 \mathrm{pM}$. The transition rate $T_{\mathrm{ci}}$ from colonies back into single cells is set at a fixed rate of $10 \%$ of $T_{\text {ic }}$. Note that these rates are not well constrained, but life-cycle analysis in the lab and field observations do report senescence of colonial cells back into flagellated solitary cells (see Fig.4-1). Colonial cells have a slightly higher $\mu_{\max }$ compared to solitary cells $\left(1.5 \mathrm{~d}^{-1}\right.$ vs. $\left.1.3 \mathrm{~d}^{-1}\right)$ to represent observations that cells living within colonies have higher gross growth grates compared to free-living, co-existing solitary cells. But solitary cells have a higher affinity (lower $R^{*}$ ) and a much higher mortality rate compared to colonies, to represent the low grazing pressure on larger, mucilaginous colonies compared to the smaller, flagellate cells. An important caveat is that grazers are not included in this iteration of the model, and could change the timing and duration of $P$. antarctica morphotype bloom dynamics if there are different size-class grazers on each life stage. Finally, the iron half-saturation constant and uptake rate is size-scaled for solitary cells $\left(r_{0}=1.5 \mu \mathrm{m}\right)$ and colonial cells $\left(r_{0}=3.0 \mu \mathrm{m}\right)$.

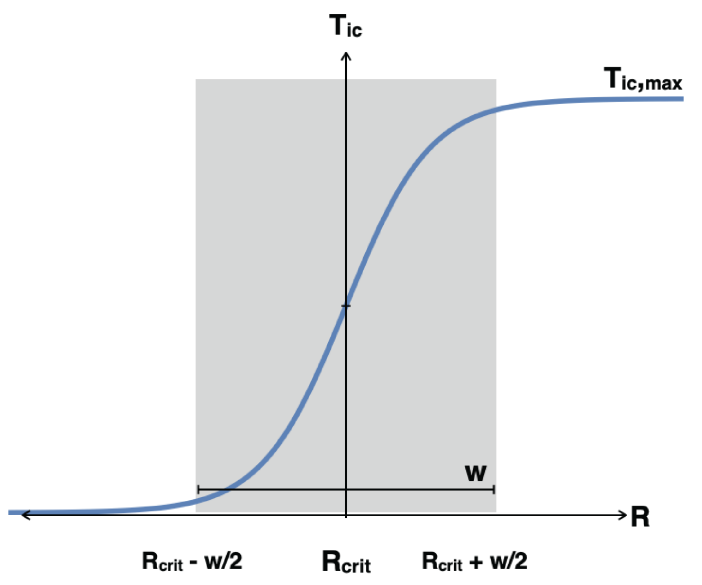

Figure 4-5: The transition function that determines the rate $T_{\text {ic }}$ of solitary to colonial cell transition, based on a critical iron concentration $R_{\text {crit }}$ and maximum transition rate $T_{\mathrm{ic}, \max }$. Flexible parameters are $T_{\mathrm{ic}, \max }, R_{\text {crit }}$, and the transition width $w$.

Diatom analogue: For this analysis, the model diatom is a $\mathrm{B}_{12}$-auxotroph that is the same size as a $P$. antarctica colony $\left(r_{0}=25 \mu \mathrm{m}\right)$. The diatom has a high $\mu_{\max }$ relative to $P$. antarctica. Its growth rate is limited by either $\mathrm{Fe}$ or $\mathrm{B}_{12}$, based on 
Monod kinetics. The micronutrient limitation status of the diatom population is calculated via Eq. (4.5). Because this diatom is a $\mathrm{B}_{12}$-auxotroph, even if there is plenty of iron, it will only grow if there is enough $\mathrm{B}_{12}$ available. Although $\mathrm{B}_{12}$-flexible polar diatoms (containing MetE and $\mathrm{MetH}$ ) exist, the model only includes an auxotroph at this stage.

We test the model at steady state and dynamic equilibrium, first focusing on $P$. antarctica morphotypes and iron, then a more complex model ecosystem which includes diatoms, heterotrophic bacteria, dissolved organic carbon, and vitamin $\mathrm{B}_{12}$. The seasonal model involves a sinusoidal iron supply rate to represent the glacial meltwater input of dissolved iron in the spring and summer, with a period of 300 days (St-Laurent et al., 2019). The iron supply rates were chosen based on 3-D global numerical model simulations from the MITgcm Darwin Ecosystem Model and personal communication with S. Dutkiewicz. The model was integrated forward in time using a Runge-Kutta 4th-order scheme to solve the system of ordinary differential equations until equilibrium. Finally, we do a sensitivity analysis of the system to test its response to iron supply rate, colony mortality rate, and diatom maximum growth rate.

\subsubsection{Prior models of coastal Antarctic marine microbial community succession}

Models of the marine microbial seasonal succession and controlling mechanisms have been developed to examine the dynamics in coastal Antarctic waters. However, to my knowledge, this is the first model to explicitly include iron and $\mathrm{B}_{12}$ dynamics as micronutrient controls on the community. A one-dimensional NPDZ model focusing on $P$. antarctica dynamics in the Amundsen Sea Polynya (ASP) under light and Fe control found that the model-data fit was most sensitive to phytoplankton growth and mortality parameters, rather than biogeochemical cycling terms for iron, and, moreover, that light was the primary limiting factor for bloom initiation, while a combination of self-shading and iron limitation caused the bloom's decline (Oliver et al., 2019). Other models of $P$. antarctica and diatoms in the Ross Sea region find that shallower mixed layer depths favor diatom growth, while earlier light availability due to reduced sea ice favors $P$. antarctica growth (Kaufman, 2017). This model, which also follows Popova et al. (2007), resolved both colonial and solitary P. antarctica, and represented the morphotype transitions (both of colony formation and solitary cell liberation) based on a threshold iron concentration and Monod-like nutrient limitation factors along with a non dimensional switch, which allows colony formation in the euphotic zone and liberation in the aphotic zone. This model resulted in an initial increase in solitary-cell $P$. antarctica which transitions to colonies under sufficient iron availability, but with the subsequent shoaling mixed layer and reduced iron supply leading to an increase in diatoms. A 3-D study of the ASP examining the meltwater pump and iron supplies was able to resolve the westward flow of organic matter due to the Antarctic coastal current flowing from the ASP to the Ross Sea, and show that highly-seasonal iron supply controls productivity along with light (St-Laurent et al., 2019). An important note is that all these prior models resolve the seasonal light 
supply and 1-D integrated light fields which are primary controlling mechanisms in their model solutions. The model presented in this chapter focuses on micronutrient dynamics and inherently assumes equal access to light for all model phytoplankton types.

\subsection{Results \& Analysis}

\subsubsection{The system at steady state}

In the case of $P$. antarctica morphotypes, the growth of solitary cells will always precede colony growth at low iron supply because smaller cells have an advantage for nutrient acquisition. The dynamics of a $P$. antarctica bloom depend on $P$. antarctica traits relative to the Fe supply rate, specifically the transition rate $T_{\text {ic }}$ from solitary to colonial cells and the critical iron threshold $R_{\text {crit }}$ that triggers colony formation. If the iron supply rate or ambient iron concentration $R$ is much less than $R_{\text {crit }}$, then solitary cells may persist but colonies will never form. If iron is enough to trigger formation at a low rate, but not enough to sustain colonies, then colonies will form but the population of colonial cells will fluctuate, as single cells continue to transition into colonies that subsequently die off. With higher iron supply, solitary cells can transition into colonial cells and colonial cells can continue to grow and coexist. At this higher iron availability, other traits like mortality and transition rates can shape the dynamics of $P$. antarctica blooms, such as the magnitude and duration of a colonial population, and hence affect the bacterial growth and a later diatom bloom. Although it has been observed that colonial cells can differentiate into swarmer cells or solitary, flagellated $P$. antarctica, the mechanisms remain unknown.

Community at steady state: In the steady-state model (Fig.4-6), the iron supply rate $S$ is fixed and the model is run until equilibrium. There is no light dependency in this model, so all dynamics are ultimately a result of the iron supply rate. A curious result is that across a range of iron supply rates, dynamics vary at equilibrium but all four model populations are able to survive. Given a sufficient (high enough) iron supply, all four populations co-exist at equilibrium because each is ultimately limited by a different resource, following Tilman's " $R^{*}$ hypothesis" (Tilman, 1981). Indeed, $P$. antarctica can be considered a single population, so there are effectively three microbial functional types ( $P$. antarctica, bacteria, diatoms) and three limiting resources $\left(\mathrm{Fe}, \mathrm{DOC}, \mathrm{B}_{12}\right)$ in the model.

Under a high iron supply (top panel of Fig.4-6), there is enough iron to support the entire ecosystem. Solitary P. antarctica cells are the best competitors for Fe due to their small size. If solitary cells persist and if there is sufficient iron to trigger the transition to colonial cells, colonial biomass can be supported. Due to low mortality and the consistent "supply" of colonial cells from the more competitive solitary cells, both morphotypes co-exist at equilibrium. The buildup of biomass results in DOC that can accumulate over time to support a bacteria population that will survive and synthesize $\mathrm{B}_{12}$. At equilibrium, diatoms are consistently $\mathrm{B}_{12}$ limited, because solitary 

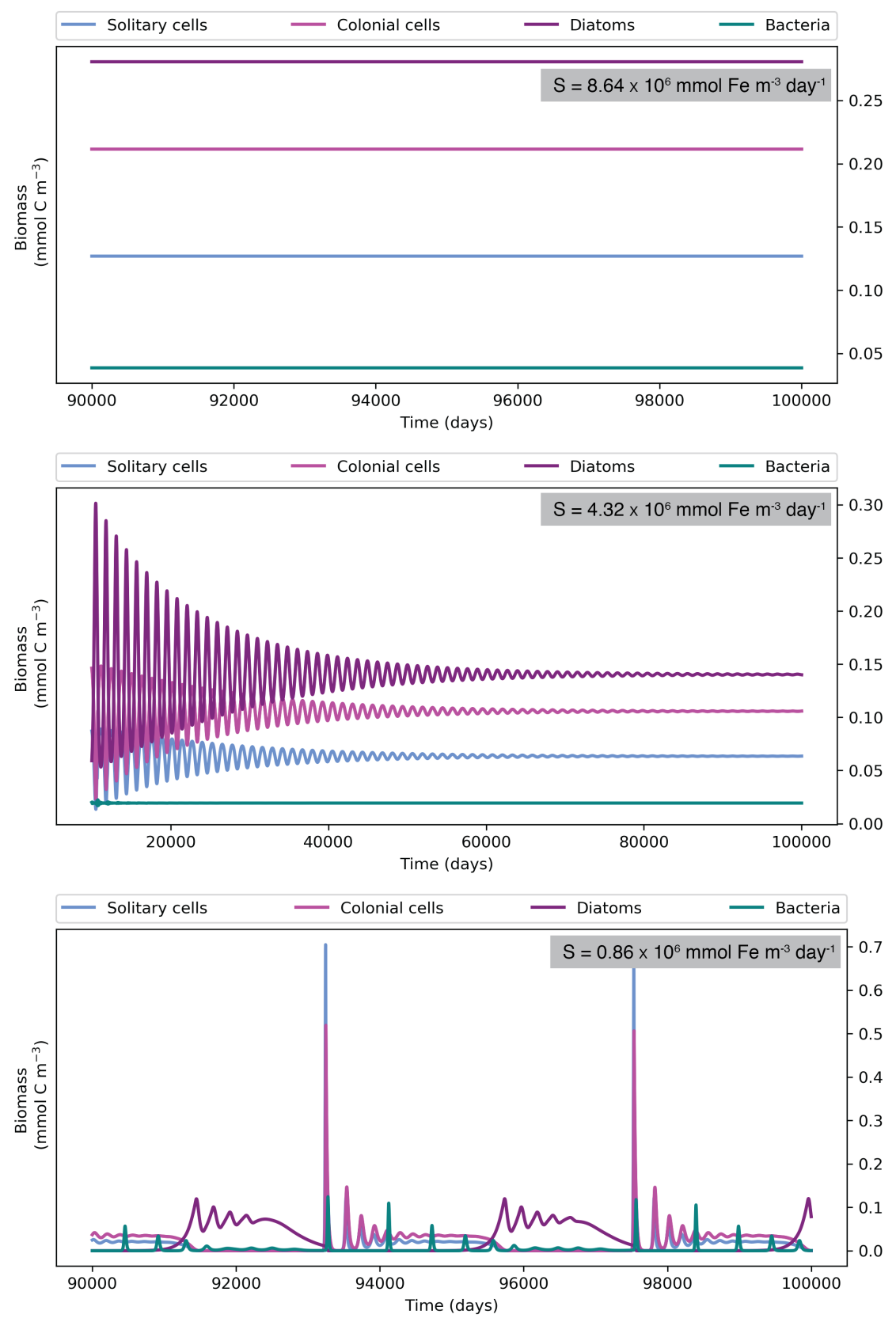

Figure 4-6: The steady-state solutions for the model Antarctic ecosystem with a fixed iron supply rate $S$. The three panels are the equilibrium solutions under a low, medium, and high iron supply rate (bottom panel to top panel). Across the range of iron supply rates, co-existence is supported at equilibrium. The top panel with the highest iron supply rate supports all microbial types with fixed biomass at equilibrium. With a lower supply rate (middle panel), the populations' biomasses are dynamically stable around an equilibrium value, with decaying oscillations. A longer time period of the model run is shown to illustrate that the oscillations dampen to a mean value. The lowest iron supply rate (bottom panel) has inherently different dynamics at steady state, with an internal complex cycle between the microbial types. There is coexistence at equilibrium at this low iron supply rates because each microbial functional type ( $P$. antarctica, bacteria, and diatom) is effectively limited by a different resource $\left(\mathrm{Fe}, \mathrm{DOC}, \mathrm{B}_{12}\right)$, and there is enough iron to support the phytoplankton populations. 
P. antarctica cells have drawn down iron to their own (lower) subsistence levels, but diatoms have a minimum $\mathrm{B}_{12}$ supply that enables them to grow off this resource and survive.

As iron supply decreases (middle panel of Fig.4-6), the coupling between populations becomes more apparent, as the biomass of each oscillates around an equilibrium value. This is the result of nutritional cross-feeding. P. antarctica Fe-limited growth allows solitary cells and colonial cells in tandem to accumulate. Their biomass is the source of detrital DOC for bacteria, which produce $\mathrm{B}_{12}$ and thereby enable the auxotrophic diatoms to persist. If the iron supply is reduced further (lower panel) a dynamic cross-feeding cycle and succession emerges, as long as there is enough iron to support solitary cell growth. This result shows how cross-feeding can lead to periodic succession even at a fixed iron supply. It is important to note that this is the quasi-steady state solution and there is not chaotic behavior, rather a complex progression between model microbial types under low iron supply rates. The equilibrium values and oscillations are specific to the parameter choices of this model. However, the inherent feedbacks result in the progression from solitary $P$. antarctica cells to colonies, then bacteria, and diatoms, even with a steady iron supply as seen in the bottom panel of Fig.4-6.

\subsubsection{Dynamic Seasonal Model}

Iron Supply: Modeling iron supply and demand in this region is hampered by a dearth of cruise data on iron concentrations and fluxes, with only discrete points in time, mostly in the spring and summer. Available data reveal that dissolved iron concentration is relatively uniform between $150 \mathrm{~m}$ to $300 \mathrm{~m}$, increasing near the sea floor due to sediment resuspension and intrusion of deep iron-rich water (Sherrell et al., 2015), and depleted near the surface due to microbial uptake. The summertime observations of dissolved iron are often complex due to heterogeneous iron inputs and biogeochemical feedbacks, particularly as a result of sea-ice melt. The conceptual model of the iron inventory in the coastal environments is that there is a winter reserve of iron due to upwelling in the pre-bloom period, then biological uptake and surface iron drawdown in the spring through summer, after which biological uptake subsides and the iron is replenished by mixing and upwelling processes (St-Laurent et al., 2019). Model studies often include iron supplies from glacial meltwater, sediments, and upwelling from deep water, and corroborate an annual pattern for iron flux to the euphotic zone, which is maximal October-December, reduces January-March, and increases again April-November (see Fig. 10, panel d from St-Laurent et al. (2019)). In this study, a sinusoidal iron supply rate (Fig.4-7) was used to represent this varied seasonal flux of iron to the euphotic zone (roughly the top $100 \mathrm{~m}$ ) in the coastal water system based on model results from St-Laurent et al. (2019).

The seasonally-forced model can reach a dynamic equilibrium with sufficient iron supply (Figs.4-8, 4-9, and 4-10). P. antarctica is Fe-limited and transitions between morphotypes depending on Fe availability. As iron supply rate increases, diatoms are initially able to grow, but are out-competed for Fe by solitary P. antarctica cells, which transition into colonies and persist due to their low mortality rates. Following the bloom of $P$. antarctica, there is a buildup of dissolved organic matter that supports 


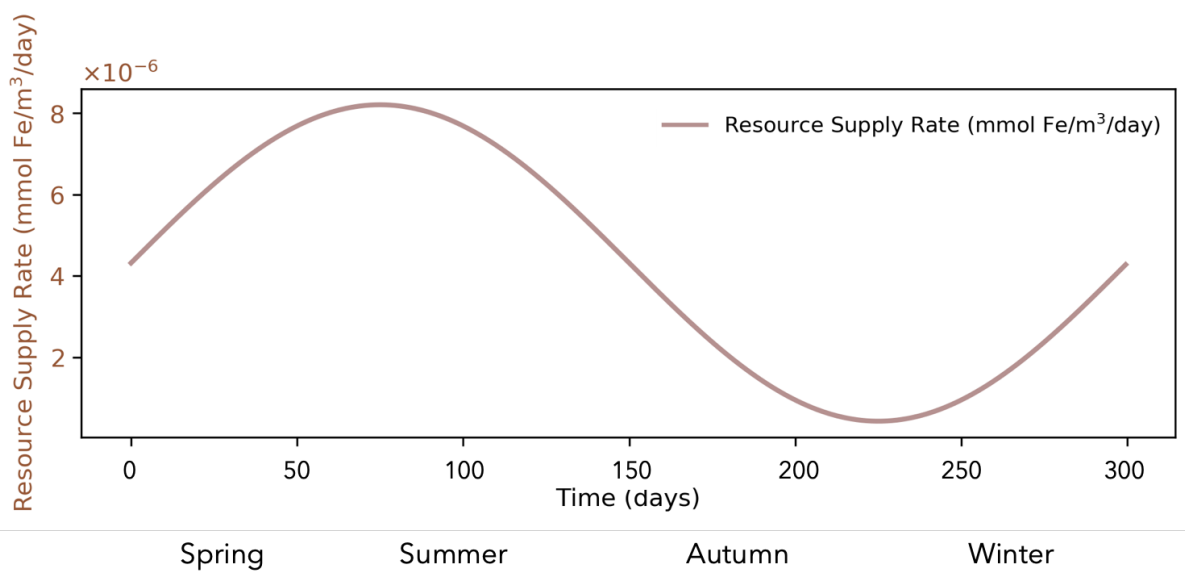

Figure 4-7: A sinusoidal iron supply represents the seasonal variation in dissolved iron supply from upwelling and meltwater sources in coastal Antarctic seas. The cycle is modeled over a 300 day period.

the bacteria bloom. The bacterial population produces $\mathrm{B}_{12}$ which then builds up in the ecosystem until it is consumed by growing diatoms. Diatom growth rate vacillate between being Fe-limited and $\mathrm{B}_{12}$-limited, as a result of their competition with $P$. antarctica for iron and dependence on bacteria for $\mathrm{B}_{12}$. The diatom population increases when iron and $\mathrm{B}_{12}$ are sufficient. Solitary cells of $P$. antarctica, however, are better competitors for iron due to their small size. The diatom populations is therefore only able to rise under $\mathrm{B}_{12}$-limited growth because they are otherwise out-competed for Fe by solitary $P$. antarctica cells. The growth dependence on a different limiting nutrient (i.e., $\mathrm{B}_{12}$ ) allows for diatoms to persist and eventually bloom, once $P$. antarctica populations wean. The high $\mu_{\max }$ of diatoms also enables a rapid response when resource concentrations are conducive to growth. Without enough primary productivity under low iron conditions, organic carbon cannot accumulate in the system to support bacterioplankton growth and $\mathrm{B}_{12}$ production, and subsequent diatom growth.

In the dynamic equilibrium presented in Fig.4-10, $\mathrm{B}_{12}$ is in the $1 \mathrm{pM}$ range, Fe is $0.1 \mathrm{nM}$ to $0.4 \mathrm{nM}$, and labile DOC ranges from 1 to $5 \mathrm{mmol} \mathrm{C} \mathrm{m}^{-3}$. Though much weight should not be placed on the exact values, the order of magnitude ranges for $\mathrm{B}_{12}$ and $\mathrm{Fe}$ are reasonable summertime values for an Antarctic coastal ecosystem like the Ross Sea. The organic carbon in this model represents labile or semi-labile DOC, and is consequently a small fraction (roughly $10 \%$ ) of measured total organic carbon in the system (Ducklow et al., 2001).

The iron supply rate was also varied to examine its effect on community dynamics. At the lowest Fe supply rate solitary cells can initially grow and transition into colonies; however, colonial cells cannot survive, and effectively become a sink for single cells. Without competition for Fe, diatoms are able to grow. But the slower buildup of DOC results in a delayed bacterial bloom that comes after diatoms instead of $P$. antarctica. With a higher iron supply rate, solitary and colonial $P$. antarctica coexist and support bacteria biomass accumulation earlier. Higher iron supply rates can 


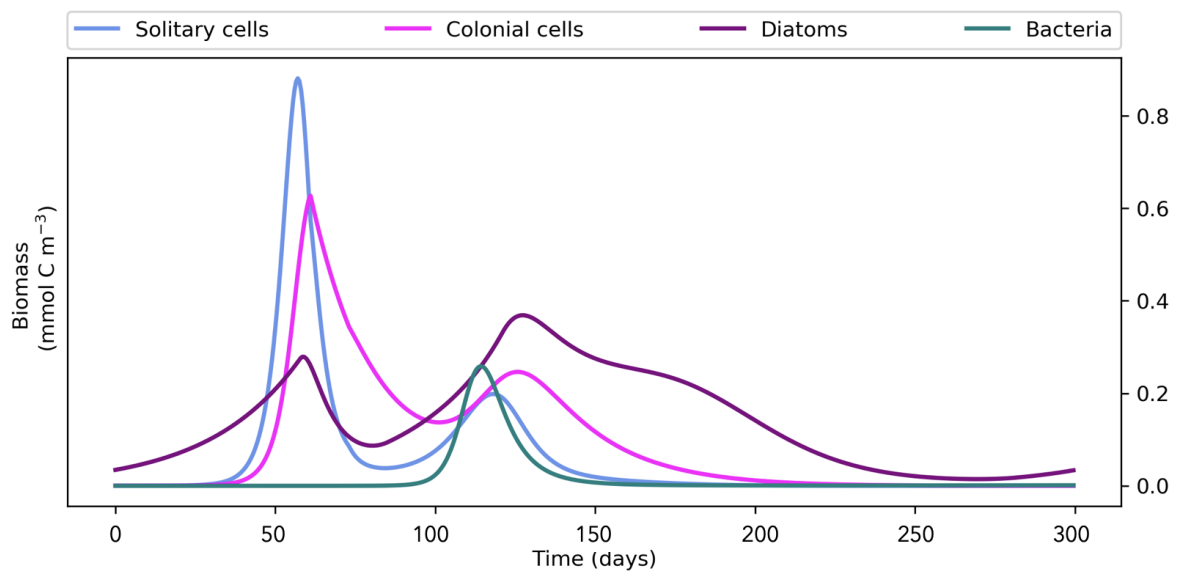

Figure 4-8: The seasonal succession of $P$. antarctica solitary cells to colonies, bacterioplankton, and $\mathrm{B}_{12}$-dependent diatoms, modeled over a single cycle of a 300 day period at quasi-equilibrium.

support the biomass of all model microbial types (see Supplemental Fig.4-12).

\subsubsection{Vitamin $\mathrm{B}_{12}$ dynamics}

This study aims to represent plausible seasonal $\mathrm{B}_{12}$ dynamics in a coastal Antarctic environment. Compared to the open ocean, productive coastal waters tends to have higher $\mathrm{B}_{12}$ concentrations, ranging from $0.5 \mathrm{pM}$ to $20 \mathrm{pM}$ vitamin $\mathrm{B}_{12}$ (Gobler et al., 2007; Panzeca et al., 2009). The low standing stock of vitamin $B_{12}$ in seawater is the result of local production, consumption, and degradation, undergoing rapid cycling that can be seasonally variable. A study that measured the $\mathrm{B}_{12}$ quota of harmful

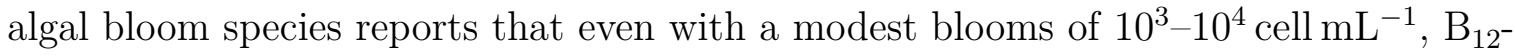
auxotrophic phytoplankton can deplete the standing stock of $\mathrm{B}_{12}$ on the order of hours to days (Tang et al., 2010). In the model, we use a high cellular $\mathrm{B}_{12}$ quota $\left(Q_{\mathrm{B}_{12}}\right)$ of $2.5 \times 10^{-6} \mathrm{pmol} \mathrm{B}_{12}$ cell $^{-1}$ (Tang et al., 2010). Taylor and Sullivan (2008) compiled halfsaturation values and found a wide range for $K_{\mathrm{B}_{12}}$ between $3 \mathrm{pM}$ to $26 \mathrm{pM}$ in temperate diatom species. A single literature value was found for the extracellular $\mathrm{B}_{12}$ produced by a heterotrophic Antarctic bacteria, with a rate of $0.02 \mathrm{nmol} \mathrm{B}_{12} \mathrm{~mol}^{-1} \mathrm{Cd}^{-1}$ from Taylor and Sullivan (2008). In marine systems, several factors contribute to the low and changing $\mathrm{B}_{12}$ concentrations, including a combination of variable $\mathrm{B}_{12}$ production and exudation from bacterioplankton, coupled with the flexible internal $\mathrm{B}_{12}$ quota between species (and luxury consumption), and photodegradation in sunlit waters. These processes make $\mathrm{B}_{12}$ a high-flux, low-concentration micronutrient across the global ocean and especially in the Southern Ocean. For $\mathrm{B}_{12}$-auxotrophic species, there may be a tight coupling between producers and consumers that modulates community composition.

Bacterioplankton: In polar surface ocean environments, the seasonal succession of bacterioplankton affects the supply of trace organics that may limit some key 


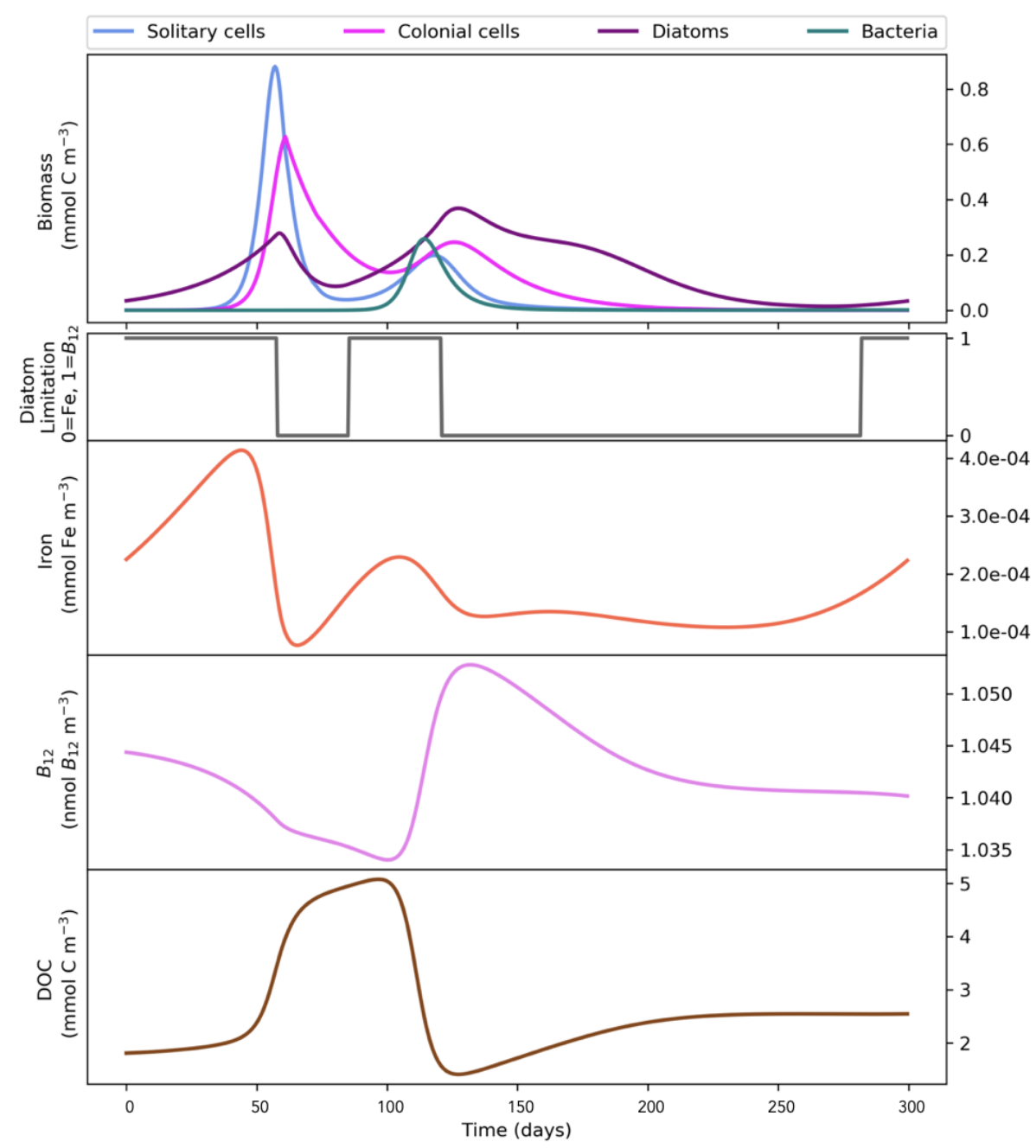

Figure 4-9: The seasonal succession of $P$. antarctica solitary cells to colonies, bacterioplankton, and $\mathrm{B}_{12}$-dependent diatoms is a result of changing iron supply rate, and the cycling of dissolved organic carbon and $\mathrm{B}_{12}$. The coexistence and pattern of succession is due to the model microbial types' limitation by distinct resources and their cross-feeding relationships. The second panel is a unit step function depicting when diatom growth rate is limited by either $\mathrm{Fe}(0)$ or $\mathrm{B}_{12}(1)$. Diatoms alternate between Fe and $\mathrm{B}_{12}$ limitation, as a result of community primary production, and bacterial growth and $\mathrm{B}_{12}$ production. The iron supply rate is sinusoidal over a 300 day period. Only one cycle at equilibrium is plotted here. 


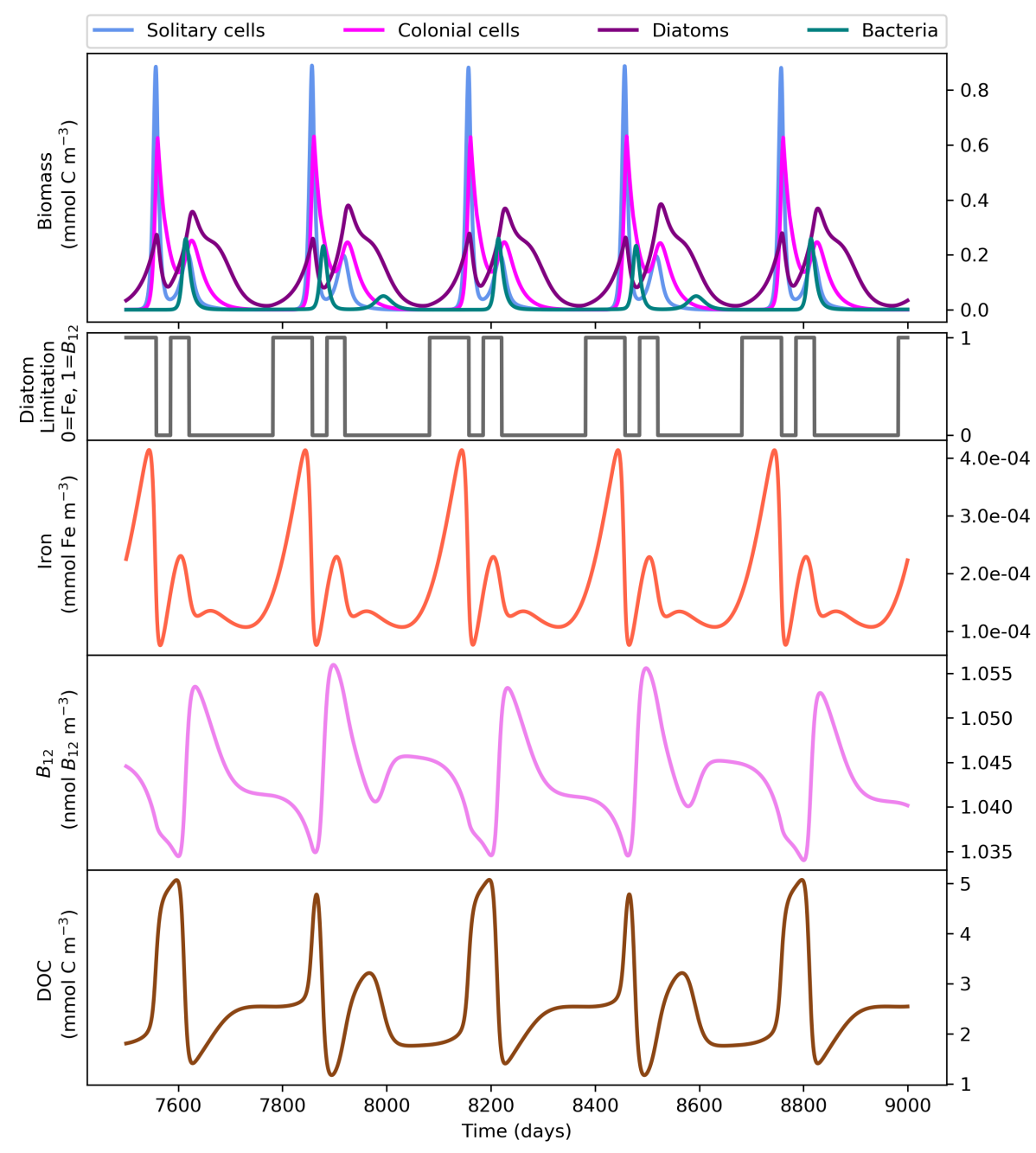

Figure 4-10: A multi-panel figure the seasonal community model results. Model results are for five years of the cycle at quasi-equilibrium. A sinusoidal iron supply rate was applied with a period of 300 days. Resource cross-feeding enables co-existence among microbial populations. The second panel depicts when diatom growth rate is limited by either $\mathrm{Fe}$ (0) or $\mathrm{B}_{12}(1)$. Diatoms alternate between $\mathrm{Fe}$ and $\mathrm{B}_{12}$ limitation, as a result of community primary production, bacterial growth, and $\mathrm{B}_{12}$ production. 
phytoplankton species. Seasonal extremes in light, temperature, and sea-ice habitat also affect the diversity and function of bacterioplankton (bacteria and archaea). Described in broad strokes, the prokaryotic community shifts from archaea-dominated in the winter to bacteria-dominated in the spring and summer, with a concurrent significant increase in total bacterioplankton cell density between winter and summer (Church et al., 2003; Murray et al., 1998). In the prolonged darkness, cold temperature, and sea-ice cover of winter, a relatively functionally diverse chemotrophic-based microbial food web dominates with archaea (Crenarchaeota and ammonia-oxidizing Archaea) (Bunse and Pinhassi, 2017; Ghiglione et al., 2012; Kalanetra et al., 2009). In the transition to spring and summer, the austral spring bloom of phytoplankton releases dissolved organic matter (DOM) that fuels heterotrophic bacterioplankton growth and a microbial loop that sustains a summertime phototrophic-based food web (Bunse and Pinhassi, 2017). In addition, phytoplankton bloom-associated bacterial communities are distinct, like Oceanospirillacaea, SAR92, and Cryomorphaceae with Phaeocystis antarctica colonies, and a range of other associations with diatom blooms or sea-ice algae communities (Delmont et al., 2014). Of relevance to this study, is the increase in $\mathrm{B}_{12}$-synthesizing bacteria and archaea that supply surface phytoplankton communities with this essential vitamin. In a blooming sea-ice edge community in McMurdo Sound, Antarctica, researchers identified bacterial species with cobalamin biosynthesis genes, with some species like Oceanospirillaceae ASP10-02a dominating the $\mathrm{B}_{12}$-biosynthesis gene abundances, having the full pathway. This species can have a preferential association with $P$. antarctica colonies and may be involved in degrading $P$. antarctica colony carbon and releasing $\mathrm{B}_{12}$ into seawater, which $\mathrm{B}_{12}$-auxotrophs or even $P$. antarctica may utilize (because it does also have $\mathrm{B}_{12}$-dependent methionine synthase enzyme) (Bertrand et al., 2011b, 2015). While much remains to be studied about the ecophysiological niches of these bacterioplankton, their seasonal shifts are evidence of a varying role in trophodynamic and biogeochemical processes. The major shifts in bacterioplankton function and abundance ultimately affect phytoplankton and carbon export, since the majority of primary production is grazed by microheterotrophs or flows through the microbial food web via consumption and remineralization by heterotrophic prokaryotes (Christaki et al., 2014; Lochte et al., 1997).

Sensitivity Analysis The model is most sensitive to phytoplankton mortality and maximum growth rate parameter values. The mortality rate of $P$. antarctica colonies must be very low in order for the colonies to persist and be competitive for iron versus diatoms. The maximum growth rate of diatoms must be greater than that of $P$. antarctica cells in order for diatoms to persist at all in the model, let alone bloom. The high maximum growth rate of diatoms $\left(2.5 \mathrm{~d}^{-1}\right)$ enables them to rapidly respond to iron and $\mathrm{B}_{12}$ availability. In the model runs presented here, the $\mu_{\max }$ of the diatoms and $P$. antarctica are relatively high compared to observations from polar regions. However, testing a range of parameter values for the sensitivity analysis shows that it is the relative differences in $\mu_{\max }$ that allow diatoms to bloom instead of being completely out-competed. Finally, the shape of the iron supply rate will also affect the timing and magnitude of the model microbial populations, although given a 
sufficient iron supply to support all the populations, the overall succession pattern is relatively stable. Finally, in the open ocean areas of the Southern Ocean that are further removed from the coastal meltwater inputs, the seasonal differences in mixed layer depth and upwelling can be important mechanisms to explore in this microbial succession context.

\subsection{Conclusion}

Here, we developed a minimal model ecosystem with mechanisms that capture the seasonal succession in Antarctic coastal marine microbial communities. We show how $\mathrm{B}_{12}$-auxotrophic diatoms can switch between primary iron and vitamin $\mathrm{B}_{12}$ limitation. The switch between Fe or $\mathrm{B}_{12}$ limitation depends on the overall system primary production and secondary production that spurs the synthesis of $\mathrm{B}_{12}$. When environmental iron supply is sufficient, diatoms can be temporarily primarily limited by $\mathrm{B}_{12}$ during the peak of iron availability. Furthermore, this model captures the overall transition of $P$. antarctica morphotypes and the persistence of colonies due to their low mortality. In the model, colonies have a very low mortality rate, which represents the combined low sinking and grazing rates. The peak in bacterial biomass lags the initial bloom of $P$. antarctica solitary and colonial cells because bacteria require an accumulation of dissolved organic carbon to support their growth. Subsequently, growing bacteria produce $\mathrm{B}_{12}$ that is excreted into the water column, and accumulates until it is consumed by auxotrophic diatoms. Without $\mathrm{B}_{12}$ as a limiting resource for these diatoms, they would be consistently out-competed by P. antarctica in this model due to its duel-phenotype nature and relatively low iron half-saturation constants. The coexistence of all microbial types at equilibrium and with dynamic forcing is possible due to each type being limited by a distinct resource (Fe for P antarctica, DOC for bacteria, and $\mathrm{B}_{12}$ for diatoms), and the supply of organic resources in a crossfeeding system. This model presents how micronutrient limitation can drive seasonal succession in Antarctic marine ecosystems, with applicability to other regions with the potential to be limited by trace organics like $\mathrm{B}_{12}$. This model also demonstrates the need to incorporate non-trophic interactions and cross-feeding/syntrophic organic resource cycling into marine microbial ecosystem models.

\subsubsection{A hypothesis for micronutrient-driven seasonal succession in Antarctic coastal waters}

A mechanistic understanding the seasonal succession of phytoplankton species in Antarctic coastal waters remains challenging to substantiate. In the Ross Sea, microbial community succession often starts with $P$. antarctica dominating the spring and early summer in iron-replete conditions $(>1 \mathrm{nM})$, followed by a bloom of bacterioplankton, then a bloom of diatom species. In this study, we present a hypothesis to explain the patterns of seasonal succession and observed micronutrient limitation based on Fe and $\mathrm{B}_{12}$ dynamics, depicted in Fig.4-11. This hypothesis could be tested both in the lab and field, using micronutrient dose-response experiments in the lab with 

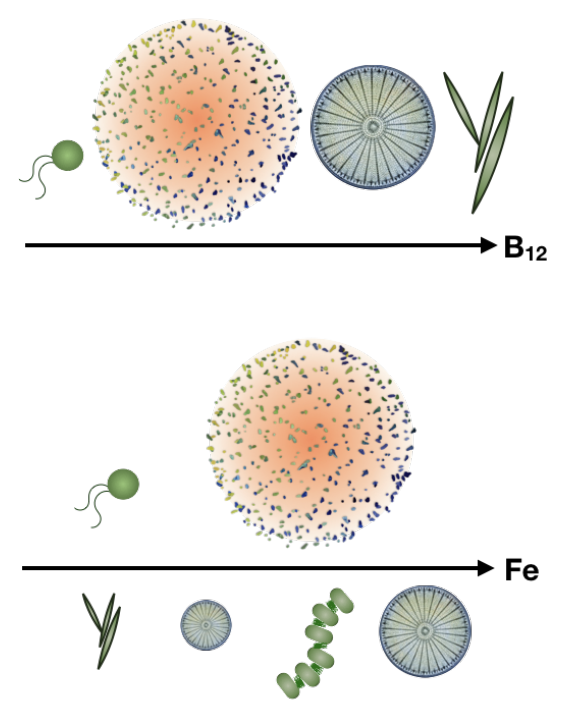

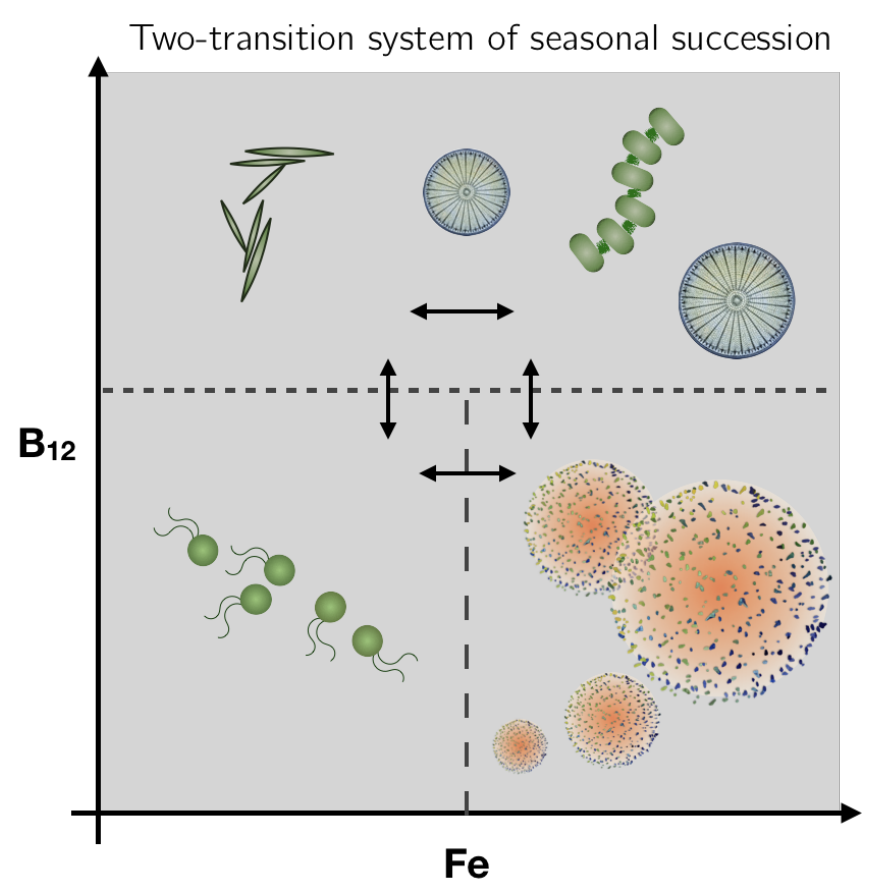

Figure 4-11: A hypothesis for micronutrient-driven seasonal succession in Antarctic coastal marine microbial communities. A two-transition system may explain a shift from early spring solitary $P$. antarctica into colonies, given sufficient iron. The growth and organic carbon produced from $P$. antarctica supports secondary production by bacteria that synthesis vitamin $\mathrm{B}_{12}$. Diatoms that are auxotrophic for $\mathrm{B}_{12}$ will then be able to grow in the summer due to vitamin-primed waters. Depending on inorganic resource supply rates and local organic resource production of $\mathrm{B}_{12}$, the system can be primarily limited by $\mathrm{Fe}$, co-limited for both micronutrients, or be primarily limited by $\mathrm{B}_{12}$ in the case of sufficient iron supply.

unialgal cultures and co-cultures of both axenic diatoms and P. antarctica and nutrient amendment experiments at sea during spring and summer conditions in a range of stations with variable Fe inputs and concentrations.

\subsection{Next steps in model development}

This model has demonstrated a plausible cross-feeding dynamic as the controlling mechanism of the observed seasonal succession pattern of $P$. antarctica, bacteria, and diatoms observed in some coastal regions of the Southern Ocean. As always, there are more details and mechanisms to examine at different scales. This discussion would be remiss if it did not include other factors that can influence community dynamics in Antarctic coastal systems. Here, I will outline the immediate next steps planned to extend this model and directions for future studies.

To start, future iterations of this model should account for a set of $\mathrm{B}_{12^{-}}$ ecophysiologies in diatoms, changes in maximum growth rate depending on use of MetE or MetH, increased competition for $\mathrm{B}_{12}$ due to uptake by bacteria and other phytoplankton. Co-existing phytoplankton with $\mathrm{B}_{12}$-dependent and $\mathrm{B}_{12}$-independent 
metabolisms are found in coastal Antarctic ecosystems. For example, two bloomforming genre, Psudonitzschia and Fragilariopsis, represent the two algal $\mathrm{B}_{12}$-strategies. $F$. cylindrus has both isoforms of methionine synthase (MetE and MetH) and is able to grow without $\mathrm{B}_{12}$, although its growth rate is likely enhanced by using the $\mathrm{B}_{12}$-dependent protein. Pseudonitzschia only has MetH and is $\mathrm{B}_{12}$-dependent, and would rely on bacterioplankton vitamin production (Ellis et al., 2017). This vitamin dependence is demonstrated in the Ross Sea $\mathrm{B}_{12}$-amendment experiments by Bertrand et al. (2007), where Fe increased $P$. antarctica growth and Fe and $\mathrm{B}_{12}$ stimulated total phytoplankton growth and especially Pseudonitzschia subcurvata, which increased from $70 \%$ to $90 \%$ of the population. In the Southern Ocean, the succession of different diatom species may be driven by differing iron and $\mathrm{B}_{12}$ needs. It is well documented that at times diatoms can also precede a $P$. antarctica bloom, perhaps by accessing an increasing meltwater iron supply or upwelled iron, having a "seed" population from the base of melting sea ice, or growing because they are also $\mathrm{B}_{12}$-flexible species. Dinoflagellates are often $\mathrm{B}_{12}$-dependent and could compete with diatoms for the vitamin. Bacteria are other important $\mathrm{B}_{12}$ consumers that compete with phytoplankton (Bertrand et al., 2015). Most bacteria also assimilate $\mathrm{B}_{12}$, even if have the capability to synthesize the vitamin. The results from Chapter 3 demonstrated that bacterioplankton also assimilate $\mathrm{B}_{12}$ and can constitute a significant portion of the $\mathrm{B}_{12}$ uptake observed in situ. It is important to also highlight that the results of Chapter 2 include multiple lines of evidence that $P$. antarctica also contains both MetE and MetH, and similar $\mathrm{B}_{12}$-related changes in $\mu_{\max }$ can be explored in this phytoplankton functional type as well. The context-dependent fitness of these $\mathrm{B}_{12}$-strategies can be explored in a seasonal model.

An important dynamic to be explored in future iterations of the model is the influence of iron on heterotrophic bacteria. In this model, we assume that iron is not limiting to bacteria, and that they are purely limited by organic carbon. However, heterotrophic bacteria may be directly and indirectly affected by iron availability in the following ways. P. antarctica morphotypes respond to iron, affecting the quality of both dissolved and particulate organic matter by $P$. antarctica solitary and colonial cells varies based on iron concentration. Organic matter from P. antarctica in highiron conditions is degraded faster than that from low-iron conditions, agreeing with other studies examining organic matter degradation from $P$. antarcticaunder nutrient stress (Becquevort et al., 2007; Verity et al., 1988). Heterotrophic bacteria compete with phytoplankton for the scarce iron in HNLC environments, and may experience Fe-limitation and access iron via different means due to siderophore production and uptake in situ. Heterotrophic bacteria require iron for respiration and ectoenzymes used to hydrolyze organic matter. Field data support that iron may limit heterotrophic bacteria in this region, with bottle Fe-amendment experiments finding an increase in both bacterial abundance and growth rates (Pakulski et al., 1996). Although heterotrophic bacteria are primarily carbon limited in the HNLC Southern Ocean, iron is secondarily limiting if there is sufficient carbon, as demonstrated by other nutrient amendment experiments (Church et al., 2000; Kirchman et al., 2000).

A slightly more complex model microbial populations that account for differences in light physiology, maximum growth rates (using MetE and MetH), a resource quota 
model, and specific grazers, can help to address other biological mechanisms that may account for the seasonal succession pattern. Of immediate interest is using a Droop model rather than Monod model to describe the iron and $\mathrm{B}_{12}$ utilization by the mode phytoplankton. The traits used in this model are fixed, but can be highly variable in natural communities depending on nutrient and light conditions. In terms of carbon flux, $\mathrm{B}_{12}$ availability, and phytoplankton dynamics, grazing is an important top-down control mechanism that has intentionally excluded from this iteration of the model, but will be explored in future work. It is well established that seasonal succession patterns in phytoplankton may be driven by predator-prey interactions and grazer specificity and is important to consider given the range of diatom sizes and the unique characteristics of $P$. antarctica colonies in this ecosystem (Sterner, 1989).

Constraining the parameters the $\mathrm{Fe}$ and $\mathrm{B}_{12}$ traits in polar $P$. antarctica morphotypes, diatoms, and bacterioplankton remains a methodological challenge, both in the lab and in models. In the case of $P$. antarctica, Fe-physiology studies have revealed that colonies have higher iron requirements and iron availability is a trigger for colony formation. (Bender et al., 2018). However, more studies are needed to understand the partitioning of iron in cells and the colony matrix. It has been suggested that the colony mucus can serve as an "external reservoir" of iron that colonial cells can access (Schoemann et al., 2005). Possibly retaining Fe in the colony matrix, like Phaeocystis does for manganese, could provide a competitive advantage in an Fe-limited system. Constraining the $\mathrm{C}$ :Fe ratio is also challenging given the partitioning between cellular and colonial carbon. Additionally, there exists a large range of interspecies variability in $\mathrm{B}_{12}$ uptake rates, half-saturation constants, and cellular quotas, especially due to luxury uptake (Droop, 2007). The maximal transition rate from solitary cells to colonial cells and back is not well constrained. These parameter values can be further examined in the model through sensitivity analysis and in the lab through controlled experiments. The variability in these trait values and functional forms can be better considered in a model with more diatom species to examine how $\mathrm{B}_{12}$ may shape community composition. The $\mathrm{B}_{12}$ quotas, uptake rates, and excretion rates of bacterioplankton require laboratory studies in order to constrain this values. In lieu of measurements, examination of these parameters in models may lend insight into a range of possible values.

The success of these large phytoplankton (diatoms and P. antarctica colonies) remains challenging to explain from the basis of nutrient competition dynamics. Scaling arguments suggest that phytoplankton with a minimal cell size and maximal surface-to-volume ratio are the most competitive in terms of nutrient uptake kinetics and reduced sinking rates. However, other mechanisms beyond steady-state nutrient competition dynamics may drive the size and competitiveness of phytoplankton in such highly seasonally variable and heterogenous environments as the Southern Ocean. For example, diatoms use non-limiting nutrients like silica to produce their frustules and increase their size and simultaneously reduce grazing pressure; and often have high growth rates, for which a trade-off with nutrient competitive ability may be advantageous in variable environments (Grover, 1990, 1991; Thingstad et al., 2005). Other strategies $P$. antarctica forms large colonies that can evade grazing pressure, remain at the surface by being near neutrally-buoyant, and potentially even store 
iron in the colony matrix (Butterfield, 1997; Ploug et al., 1999). Polar diatoms and $P$. antarctica colonies may even have larger storage capacity and internal quotas of limiting nutrients that provide an advantage (Verdy et al., 2009). Future work on this model can include examining the impact of various $P$. antarctica colony advantages beyond a reduced mortality rate.

Seasonal patterns of iron supply from upwelling, glacial melting, and circulation and currents in the coastal seas are important to resolve in order to more accurately capture microbial biomass, iron concentrations, and temporal dynamics. As a start, modeling the mixed layer dynamics is important to capture ambient light levels, upwelled iron supply, and address the existing paradigm that mixed layer depth influences community succession due to the sinking of dense diatoms and floating of $P$. antarctica colonies. It is challenging to represent all these key processes in a $0-\mathrm{D}$ or even 1-D model of this ecosystem. For one, the glacial melt rates around the continent vary, with the Amundsen Sea Polynya experiencing much higher melt rates compared to the Ross Sea Polynya (Rignot et al., 2013). In addition, iron-ligand chemistry and the potential flux from particulate iron have not been included in this model, but are important to consider for the overall trace metal biogeochemical cycling. Finally, recycling of iron in the summer mixed-layer is an important mechanism that may help sustain the microbial productivity during period of reduced iron input (Tagliabue et al., 2014). Implementing these model marine microbes in a spatially resolved regional or global biogeochemical model can help to provide a more complete iron cycling system. A major unknown is the seasonal iron supply across coastal Antarctic regions due to a lack of a resolved time series measurement in this expansive systems. While broad iron supply patterns can be inferred from existing studies and known mechanisms, the variability in iron supply and even which sources are most important to local communities is poorly constrained. Further work is needed to extend this model and further constrain the dissolved iron and vitamin $\mathrm{B}_{12}$ dynamics in coastal Antarctic regions beyond the scope of this model, since most biogeochemical models do not include cobalt as a limiting resource and also do not resolve all the potential iron supply routes to coastal Antarctic marine systems.

The model presented in this chapter is a starting point for investigations into a more complex set of community dynamics. By demonstrating that cross-feeding interactions and nutritional dependencies can enable coexistence and drive a seasonal succession, this study hopes to inspire future work into micronutrient and trace organic controls on Antarctic marine microbial communities in a climate-critical region of our planet. Given the challenges to sample this location and the lack of highly resolved time series, biogeochemical models are a useful way to test our understanding of the controlling mechanisms of seasonal succession. Models also enable us to test hypotheses and key mechanisms, integrate our understanding of complex systems, and help guide field expeditions and laboratory experiments. 


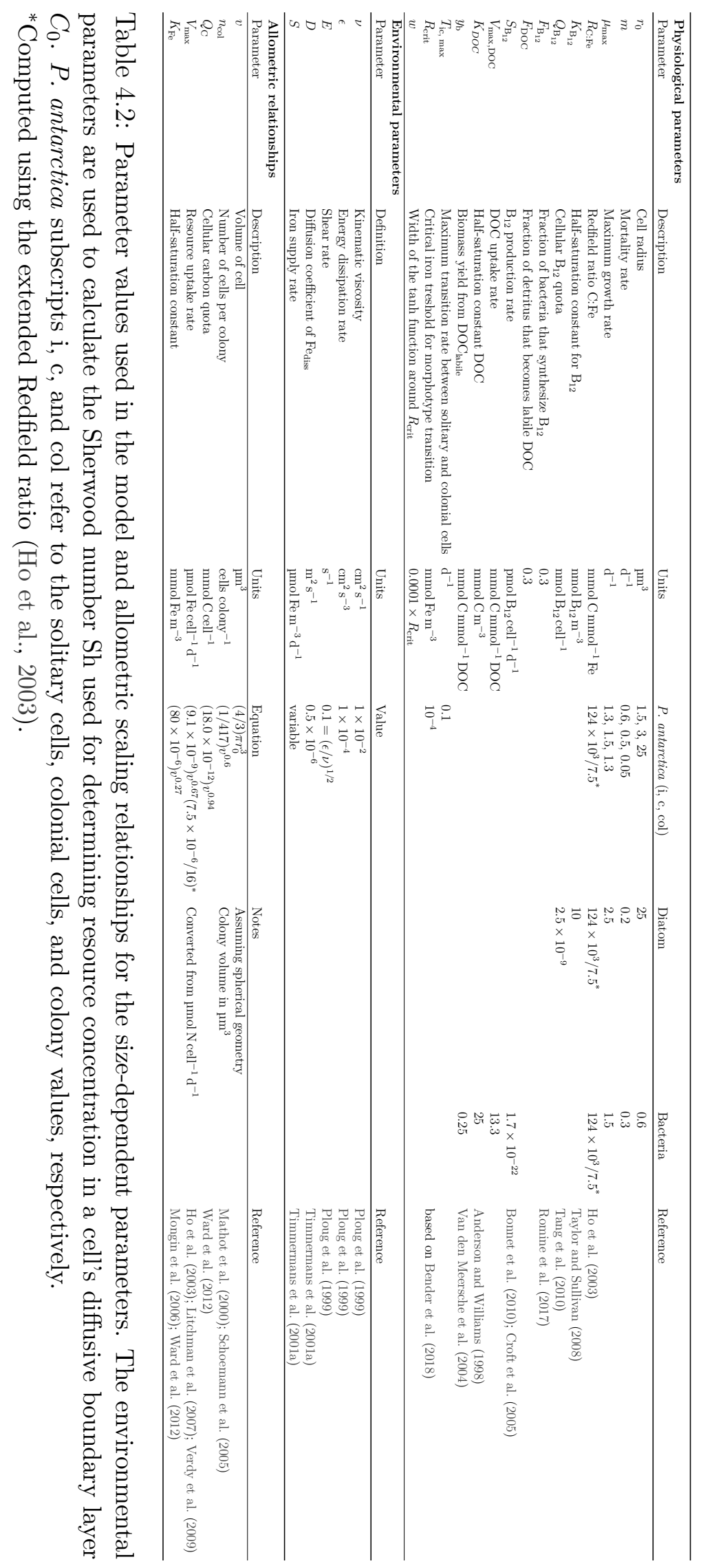




\subsection{Supplemental Figures}
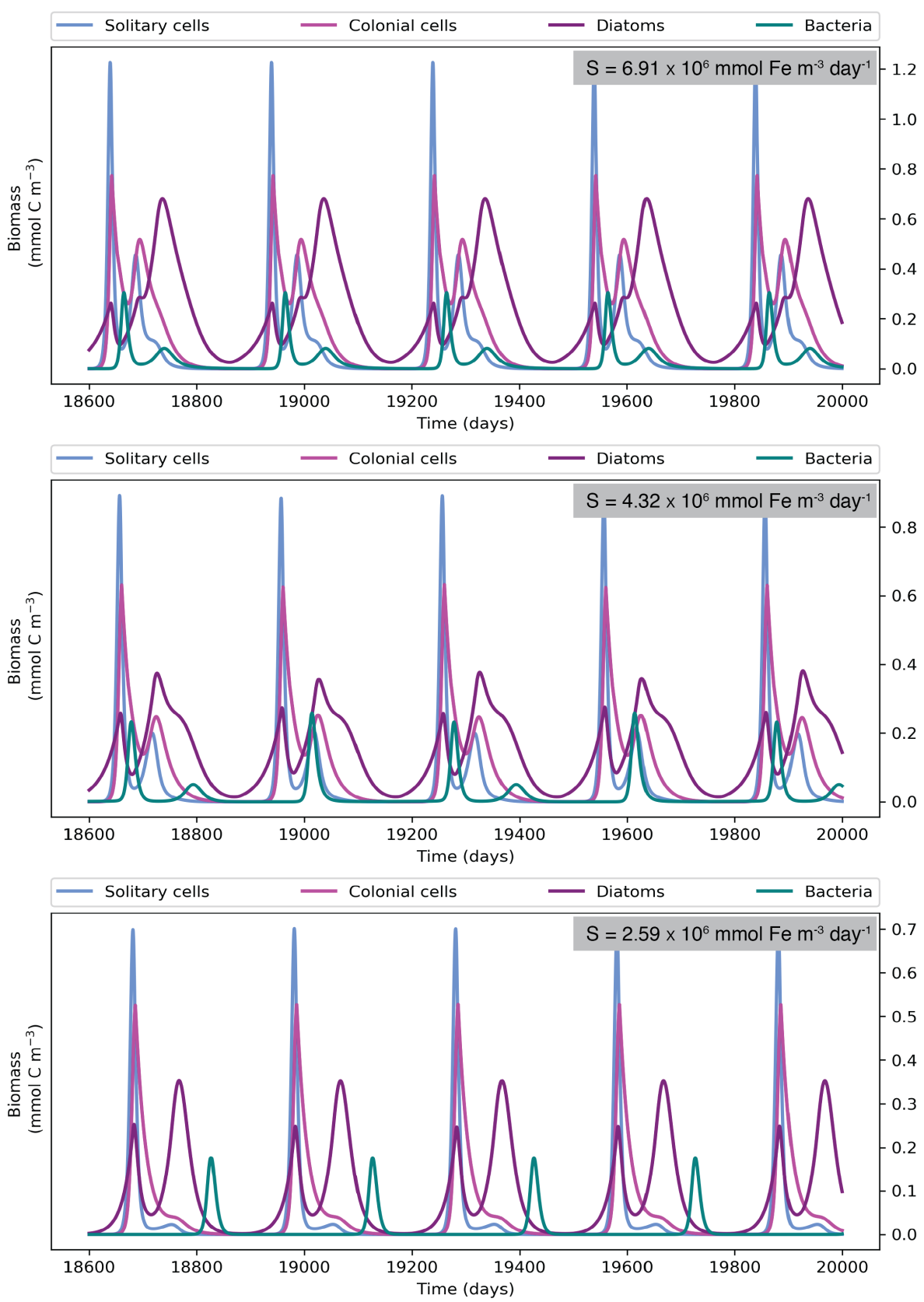

Figure 4-12: Iron supply rate modulates both the magnitude and shape of $P$. antarctica and diatom blooms, as well as the timing of the bacteria bloom. Results are discussed in the text. 


\section{Chapter 5}

\section{Conclusion and Future Directions}

This dissertation has provided insights into key aspects of microbe-driven cobalamin cycling in Antarctic coastal marine ecosystems. A multi-scale research approach enabled the characterization of the $\mathrm{B}_{12}$-ecophysiology of a strain of $P$. antarctica, the measurement of community cobalt and cobalamin uptake rates in Antarctic marginal seas, and the development of a numerical ecosystem model to test a hypothesis of micronutrient-driven seasonal succession in coastal Antarctic marine microbial ecosystems. Each of these methods addressed the system at a different scale, but complemented one another to build towards a more comprehensive understanding of this important ecosystem. Here, the main conclusions found in each study and their broad implications are summarized along with a discussion of future research directions.

\subsection{Thesis Overview}

Antarctica and the Southern Ocean that encircles this frozen continent are critical to the homeostasis of Earth's current climate. The Antarctic region buffers against the effects of human-driven climate change due its ability to sequester atmospheric carbon dioxide $\left(\mathrm{CO}_{2}\right)$ in the deep ocean via the solubility pump and biological pump. Frigid temperatures result in the formation of sea ice and the sinking of cool, briny, dense seawater that removes $\mathrm{CO}_{2}$ from the atmosphere and drives Antarctic thermohaline circulation. In addition, seasonally high primary production by phytoplankton draws down atmospheric $\mathrm{CO}_{2}$ via photosynthesis and sequesters this carbon in the deep ocean via vertical transport of sinking dead cells. Despite its vital role in maintaining climate, this region is particularly sensitive to the impacts of global warming. The predicted (and ongoing) changes of increased warming, ocean acidification, shallowing mixed layer depths, stronger winds, increased light and UV exposure, declining sea ice, freshening surface waters, and alterations to patterns of upwelling and costal nutrient supplies, will conspire to reduce the capacity of the Southern Ocean to absorb atmospheric $\mathrm{CO}_{2}$, offset human impacts, transport heat, and drive the global ocean conveyor belt (Deppeler and Davidson, 2017).

This suite of environmental changes directly affects the microbial base of the 
Antarctic food web that has evolved for millions of years to cope with and even thrive in sea ice habitats, cold waters, and the large seasonal swings in temperature and light. It is particularly important to study, understand, and predict the response of keystone coastal Southern Ocean marine microbes. Due to their proximity to the Antarctic coastline and shelf waters, these communities will be the bellwethers of climate change in the Antarctic region because they will respond to increasing meltwater inputs, shoaling mixed layer depths, and warming surface waters, among all the other expected environmental changes. The primary production of phytoplankton and the nutrient cycling of bacterioplankton in the microbial loop form the foundation that supports microscopic zooplankton, krill, fish, seabirds, penguins, seals, whales, and increasingly humans. Human activity in the Antarctic region has increased due to ecotourism and commercial fishing, especially of Antarctic krill that is harvested in massive quantities. Although this biome may be less biodiverse than its temperate and tropical counterparts, it makes up for its lack of diversity in sheer number of individuals and their metabolic flexibility.

Knowing how Antarctica's endemic microfauna will likely respond to climate change is necessary to evaluating ecosystem and biogeochemical changes to this region that influence its ability to buffer against continued human greenhouse gas emissions. However, constraining carbon export and investigating marine microbial communities is challenging in this region due to its remote location, extreme climate, heterogeneous environments, and logistical difficulties that limit high-resolution sampling. Antarctic waters are challenging to access and study, thus an interdisciplinary approach is needed to combine methods at discrete scales in combination with mechanistic detail and ecological theory to arrive at a more holistic understanding of the ecosystem.

A major unknown in Antarctic microbial ecology is how phytoplankton will respond to warming waters and changing nutrient supplies, especially of the region's primary limiting resources: iron and vitamin $\mathrm{B}_{12}$. The timing, location, and dominant species of phytoplankton blooms affects the region's potential to sequester atmospheric carbon via the biological pump; notably, $P$. antarctica is significantly more carbon rich relative to diatoms and thus has a higher potential to export carbon to the deep ocean (Arrigo et al., 1999). Therefore, an understanding the underlying environmental and ecological controls on phytoplankton community composition is essential to evaluate and predict ecosystem-scale processes like primary production, carbon export, and nutrient cycling. Furthermore, given the region's propensity for $\mathrm{B}_{12}$ limitation, characterizing the nutritional inter-dependencies between phytoplankton and bacterioplankton is of interest because prokaryotes depend on dissolved organic matter (DOM) from phytoplankton photosynthate, exudate, and detritus, and some phytoplankton have an absolutely requirement for vitamin $\mathrm{B}_{12}$ for growth, which ultimately comes from bacteria and archaea.

Several key questions remain to be addressed to improve our baseline knowledge of Antarctic coastal marine ecosystems. Which environmental or ecological factors are the dominant controls on community composition? Are sea ice microbial communities necessary to "seed" primary production in the spring? How will bacterioplankton and phytoplankton activity change with warming temperatures? Will increased glacial and sea ice melt significantly change the iron supply to surface marine microbial 
communities? At what concentrations will the community be primarily limited by iron, co-limited by iron and $\mathrm{B}_{12}$, or potentially primarily limited by $\mathrm{B}_{12}$ ? Do phytoplankton require a direct symbiosis with bacteria to acquire sufficient concentrations of $\mathrm{B}_{12}$ or is enough available in the environmental for their metabolic needs? What are the $\mathrm{B}_{12}$ requirements of key polar microbial species? What are the rates of $\mathrm{B}_{12}$ synthesis and exudation by polar bacteria and archaea? How do we best represent phytoplanktonbacterioplankton interactions in marine microbial ecosystem models? These questions are all exciting areas for curious scientific minds to investigate.

The overarching goal of this dissertation was to contribute to the characterization of microbe-driven cycling of cobalamin in Antarctic coastal seas with targeted laboratory studies, field measurements, and a numerical ecosystem model, to elucidate how the exchange of organic resources between bacterioplankton and phytoplankton underlie primary production and community dynamics. I used three different approaches to tackle the complex question of how trace metals and trace organics affect the marine microbial ecology of Antarctic coastal ecosystems. Each of these three approaches enables us to understand microbial ecophysiology, nutrient-exchange interactions, and feedbacks on biogeochemistry at a different spatio-temporal scales; and consequently, each approach also has its limitations. What follows next is a summary of the guiding research questions for each study, the scientific findings, broader implications, and directions for future research.

\subsection{Dissertation Findings}

What is the $\mathrm{B}_{12}$ ecophysiology of they keystone Antarctic phytoplankton species Phaeocystis antarctica? Furthermore, do its distinct morphotypes have different $\mathbf{B}_{\mathbf{1 2}}$ needs? Chapter 2 focused on describing the ecophysiology of $P$. antarctica morphotypes under a wide range of $\mathrm{B}_{12}$ conditions and low and high iron concentrations to induce predominantly solitary cells or colonial cells in culture. This study was motivated by a dearth of laboratory studies of $P$. antarctica morphotypes to multiple, simultaneous nutrient stressors and a lack of experiments to asses the micronutrient requirements of this critical Antarctic species. A recent study by Nef et al. (2019) used bioinformatics analysis to asses the $\mathrm{B}_{12}$ requirements of $P$. antarctica. However, transcriptomic databases of algal cultures reflect the gene expression patterns of alga grown under specific conditions. Most algal cultures using either artificial or supplemented seawater have media that is replete in $\mathrm{B}_{12}$, perhaps even in excess since the typical F/2 media uses 100 pM of cobalamin, which is roughly 100 times more than the standing stock in seawater. Under replete $\mathrm{B}_{12}$ conditions, it is expected that $P$. antarctica would express the $\mathrm{B}_{12}$-dependent methionine synthase.

Global proteomic and key pathway changes are discussed in detail in the chapter, while this conclusion focuses on the significance of the methionine synthase isoforms measured by transcriptomics and proteomics. $P$. antarctica morphotypes have a flexible metabolism that allows them to adjust to variable iron and $\mathrm{B}_{12}$ conditions. $\mathrm{A}$ larger proportion of colonial cells were present under the higher iron treatment. Growth rates were maximal for predominantly solitary cells under low iron conditions in the 
range of environmental standing stocks of $\mathrm{B}_{12}$; whereas, growth rate was stable for the predominantly colonial cells under high iron conditions, except a notable increase in growth rate in the highest $\mathrm{B}_{12}$ treatment. Mass spectrometry derived protein data showed that at the highest $\mathrm{B}_{12}$ concentrations, the $\mathrm{B}_{12}$-dependent methionine synthase isoform (MetH) was utilized in the cultures, while the $\mathrm{B}_{12}$-independent isoform (MetE) was present in all treatments but significantly differentially abundant under lower $\mathrm{B}_{12}$ conditions. This study clearly demonstrated that both solitary and colonial $P$. antarctica cells are not $\mathrm{B}_{12}$-dependent and indeed regulate their methionine synthase isoforms according to $\mathrm{B}_{12}$ availability. While the MetH protein has been previously observed, we identified a putative MetE protein that may be a novel isoform that had been overlooked by other bioinformatics-based methods because it differs from the canonical MetE in E. coli and model marine diatoms. A recent study demonstrated a new "core-MetE", suggesting that it is plausible that multiple MetE proteins exist in nature (Deobald et al., 2020).

This discovery raises several questions that are ripe areas for future work. First, the protein structure and functional characterization of this putative MetE should be determined in the lab, versus inferences in silico. The phylogeny of this putative MetE protein revealed that sequence is found in the transcriptomes of other $P$. antarctica strains, members of the haptophyte lineage, dinoflagellates, cryptophytes, stramenopiles/heterokonts, and bacteria. The curious similarity of these eukaryotic algae having polymorphic life cycles or flagellated life stages requires further investigation into the role of MetE (particularly this putative MetE) in the life history of these species. Future studies are directed to examine the putative MetE protein in more polar phytoplankton species, even at the strain-level resolution. The putative MetE protein was identified via proteogenomics (Nesvizhskii, 2014), whereby proteomic data from mass spectrometry results provided protein-level evidence of gene expression; and that sequence was used to investigate the sample transcriptomic assembly in silico to refine the gene model. Characterizing this putative MetE protein is possible through over-expression studies in model algal taxa or in E. coli to study the function of this putative MetE (primarily to assess if it can overcome $\mathrm{B}_{12}$ limitation in MetE knockouts) and identify its protein structure. Strain-level differences exist in the $\mathrm{B}_{12}$-auxotrophy of phytoplankton species, so due diligence is needed to examine the $\mathrm{B}_{12}$-ecophysiology of $P$. antarctica strains as well as other Phaeocystis species before broad claims on phylogenetic relationships can be made. Finally, this putative MetE sequence can be searched for in existing metaproteomic and metatranscriptomic databases of samples from across the global ocean to see if it is detectable and differentially abundant in certain regions or environmental conditions. An immediate next step would be to examine the metaproteomics samples collected during the CICLOPS research expedition to examine its presence in Antarctic seawater communities.

The results of this chapter demonstrate the flexible $B_{12}$-ecophysiology of the keystone polar phytoplankton $P$. antarctica, through a combination of growth assays, transcriptomics, targeted and global proteomics, proteogenomics identification of a putative MetE protein and phylogenetic analysis of its presence in a range of eukaryotic algae and bacterioplankton. These findings are an important advancement 
to understanding the micronutrient limitation of Antarctic coastal ecosystems and add necessary species-level understanding of the vitamin ecophysiology of $P$. antarctica. These results corroborate the observations of incubation amendment experiments and the regional and seasonal patterns of $P$. antarctica blooms in this area. This study also highlights the need for experimental validation of bioinformatics analyses and the continued improvement of references databases. Finally, the knowledge that $P$. antarctica is not auxotrophic for $\mathrm{B}_{12}$ was essential to interpreting prior field experiments and the measurements in Chapter 3.

What environmental or ecological factors control the rates of $B_{12}$ and cobalt uptake in natural seawater communities? Chapter 3 moved from the lab-based examination of $P$. antarctica physiology towards investigating the in situ community uptake rates of cobalt and cobalamin. In this field-based study, which sampled Antarctic coastal and polynya waters from the Amundsen Sea, Ross Sea, and Terra Nova Bay, I used ${ }^{57}$ Co radiotracer incubation experiments to measure the concurrent uptake of $\mathrm{Co}$ and $\mathrm{B}_{12}$ into natural seawater microbes. These samples were collected on $0.2 \mu$ mand $3 \mu$ msized filters to capture the uptake on the total community and eukaryotic phytoplankton, respectively. The radiochemical uptake rate measurements constitute the largest dataset of its kind in the Antarctic, indeed in any oceanographic region. A total of fifteen stations were sampled from the surface to depth, with hundreds of uptake rate measurements between the stations, depths, and filter size fractions. Coupled with hydrographic data, chemotaxonomic phytoplankton pigment data, and trace metal concentrations, this dataset enables the comparison of concurrent cobalt and $\mathrm{B}_{12}$ uptake rates.

The overall findings of this study are that $\mathrm{Co}$ and $\mathrm{B}_{12}$ uptake rates are highest in the top $100 \mathrm{~m}$ of the water column, where the bulk of the phytoplankton community is concentrated. The highest rates of uptake of $\mathrm{B}_{12}$ were in of warmer, fresher water masses, indicating regions of sea ice or glacial meltwater input. Furthermore, the regionally separated uptake rates of both cobalt and $\mathrm{B}_{12}$ were significantly correlated with diatoms (with biogenic silica and fucoxanthin as the proxies) and were not correlated with $P$. antarctica (with 19-hexanoyloxyfucoxanthin). Again, these findings were consistent with prior nutrient amendment experiments in the Ross Sea, wherein diatoms responded to the addition of $\mathrm{B}_{12}$, whereas $P$. antarctica responded to the addition of iron when it was limiting in situ (Bertrand et al., 2007; Sedwick et al., 2000). This dataset validates our understanding that phytoplankton drive the uptake of $\mathrm{Co}$ and $\mathrm{B}_{12}$ and the diatoms are likely primarily responsible for the bulk of this uptake. Bacterioplankton also contribute substantially to total uptake and are important competitors for these micronutrients as well. The ecological and environmental correlations identified in this chapter lay the groundwork for more in-depth investigations of which diatom species are driving the observed uptake rates in different environmental contexts.

Though these field measurements cover a wide range of geographic locations and microbial communities are still limited in their temporal and spatial scope. Furthermore, although concurrent water samples were collected to measure $\mathrm{B}_{12}$ 
concentrations, these measurements are yet to be determined. In Chapter 3, I calculate estimated in situ $\mathrm{B}_{12}$ uptake rates based on a presumed concentration of $1 \mathrm{pM}$. As both total cobalt and iron concentrations are measured from CICLOPS data, we will be able to compare the uptake rates to regions of potential iron limitation. Finally pending cruise metatranscriptomes and metaproteomes will allow for a richer examination of cobalt- and $\mathrm{B}_{12}$-related genes and proteins to draw connections regarding which species are responsible for the bulk of the radiochemical uptake and under what influencing environmental conditions. These uptake rates serve as a baseline dataset that can be use for future comparisons and benchmarking parameter values and uptake rates in cobalt biogeochemical models, particularly of the Antarctic region. Future investigations can build on this dataset to collect a timeseries of more highly-resolved cobalt and cobalamin uptake measurements in select locations in the Amundsen Sea and Ross Sea. The significant correlations between uptake rates (of Co and $\mathrm{B}_{12}$ ) lend support to the development of a $\mathrm{B}_{12}$-auxotrophic model diatom for use in ecosystem models exploring Anatrctic marine microbial communities, like that presented in Chapter 4 .

Do micronutrients drive the observed seasonal succession in Antarctic seas? The findings in Chapters 2 and 3 motivated a reexamination of the existing paradigm of microbial seasonal succession in Antarctic coastal environments in Chapter 4. In the chapter, I present a hypothesis for micronutrient-driven seasonal succession and a numerical model to test the underlying assumptions of iron and $\mathrm{B}_{12}$ affecting the phytoplankton community. Prior to this dissertation, the paradigm of microbial community development was based on iron supply, light levels, and mixed layer depth (Arrigo et al., 1999). The hypothesis and model results presented here posit that the primary limiting resource for phytoplankton is iron and for bacterioplankton is dissolved organic matter (DOM), and with the onset of springtime melting the dissolved iron supply increases and fuels the initial growth of $P$. antarctica and transition from solitary to colonial cells, which allows for the buildup of detritus and DOM that spurs bacterioplankton growth and $\mathrm{B}_{12}$ production, which ultimately allows $\mathrm{B}_{12}$-depependent diatoms to grow instead of being outcompeted by $P$. antarctica for iron alone. The model results demonstrate that competition for inorganic resources, loose cross-feeding interactions, and co-limitation for $\mathrm{B}_{12}$ can enable the stable coexistence of multiple microbial types and may result in the observed seasonal succession patterns in this region.

This hypothesis challenges the existing paradigm of inorganic resource (iron) and environmental controls on Antarctic marine microbial community progression. Researchers have questioned the validity of this paradigm with recent work describing the rapid photo-acclimation and similar photosynthetic efficiencies of $P$. antarctica and diatoms, the formation of expansive $P$. antarctica blooms along the Amundsen Sea coast in shallow mixed layers presumed to select for diatoms, bacterioplankton blooms that follow $P$. antarctica blooms, and the seasonal changes in trace metal input and bacterioplankton activity, beyond physical forcings alone (Delmont et al., 2014; DiTullio et al., 2000; Ducklow et al., 2001; Noble et al., 2013; Sedwick et al., 2000). 
Although there is a lack of a singular time series observational dataset, a thorough review of the literature yields studies collected at different times that paint a consistent picture of the seasonal succession pattern, as described in Chapter 4. While other studies have examined an iron-threshold transition between solitary and colonial $P$. antarctica (Kaufman, 2017; Wang and Moore, 2011), this is the first ecosystem model to my knowledge that includes the production of $\mathrm{B}_{12}$ by bacteria and consumption by a $\mathrm{B}_{12}$-auxotrophic diatom model type as other controlling mechanisms. Other dynamics can be explored in future iterations of the model including: competition for iron and $\mathrm{B}_{12}$ by bacteria, inclusion of zooplankton grazers, a diversity of $\mathrm{B}_{12}$-strategies in model diatoms, a switch from Monod kinetics to a Droop (quota) model for $\mathrm{B}_{12}$, and the inclusion of other mechanisms like iron storage in the colony matrix and bacterial symbionts associated with both phytoplankton types that offer a "direct" $\mathrm{B}_{12}$ source. Finally, the changing iron supply rate can be provided by model output for a more realistic and variable iron supply through the year.

An immediate and feasible next step is to examine the consequences of including $\mathrm{B}_{12}$ limitation in marine microbial ecosystem models to further develop hypotheses that can be tested in the field and through laboratory experiments. An important next step is the design of a phytoplankton functional type model of the Phaeocystis genus for the MITgcm Darwin ecosystem model, because currently this genus is not represented. Once included, it will be possible to examine the competition dynamics between $P$. antarctica solitary cells, colonies, and diatoms for dissolved iron. Further steps include integrating bacterioplankton DOM and $\mathrm{B}_{12}$ dynamics and ultimately a range of diatoms with differing $B_{12}$ strategies (dependent and flexible) to examine how feedbacks inherent the model will result in the emergent community structure and seasonal dynamics in the simulated coastal Antarctic seas. The expansion of the model presented in Chapter 4 to a global ecosystem model will allow for a spatio-temporal analysis that is needed to examine the possibly underlying drivers of phytoplankton blooms in the heterogenous environment of the coastal Southern Ocean.

This chapter has a broader significance in the field of marine microbial ecology and modeling of microbial oceanography, especially of polar microbes, because it illustrates that non-trophic interactions can result in complex and ecologically relevant dynamics in model marine microbial communities. The inclusion of trace organics and loose cross-feeding interactions in large-scale models is a challenging endeavor. It is my hope that through this study, I have engendered more interest and shown the relevance of considering and carefully including a range of phytoplankton-bacteria interactions in large scale ecosystem models.

\subsection{Broader Implications and Future Outlook}

Altogether, this dissertation provides important insights into the microbe-driven cycling of cobalamin in Antarctic seas. The multi-scale approach used in this dissertations allows for a more holistic understanding of the $\mathrm{B}_{12}$-related metabolism of keystone Antarctic phytoplankton species and how trace organics can influence the composition and dynamics of seawater microorganisms in varied Antarctic marine environments. 
The results of these studies will inform future work on the micronutrient drivers of Antarctic marine microbes and the biogeochemical cycling of cobalt and cobalamin. Vitamin $\mathrm{B}_{12}$, with its uniquely prokaryotic origins and complex pathways through microbial communities can be a model trace organic to examine the role of non-trophic phytoplankton-bacterioplankton interactions in the global ocean. Novel approaches to modeling $\mathrm{B}_{12}$ dynamics in microbes are needed because the standard toolbox of Redfield stoichiometry, Monod kinetics, and environmental resource supply are insufficient to capture the physiological details and mechanisms involved in the uptake, excretion, associations, and use of this essential metabolite.

Scientists face the exacting challenge of studying this formidable environment, understanding its underlying drivers, and predicting the net effect of multiple, concurrent climate-induced stressors on the complex Southern Ocean biome. Based on the findings in this dissertation, microbial oceanographers should continue expanding research into the role of trace organics as limiting resources and influencers of community composition and the non-trophic microbial interactions that are fundamental to marine microbial systems. Nutritional inter-dependencies affect not only the structure and function of microbial communities, but also their stability and resilience to climate change. The ultimate goal for future work should be to connect cobalamin cycling at the microbial scale to ecosystem and biogeochemical scale processes and global climate.

\section{Final Thoughts}

The research conducted in this dissertation was pivotal to my growth as a scientist. Thanks to the MIT and WHOI community, especially the members of the Follows Lab and Saito Lab, I was able to gain the tacit knowledge and experience necessary to tackle problems in this complex and fascinating area of research. From the members of the Saito Lab at WHOI, I learned how to design and implement trace metal clean algal culture experiments, grow and monitor P. antarctica solitary cells and colonies, prepare culture samples for transcriptomics and proteomics analyses, and delve into the bioinformatics analysis pipelines to uncover key metabolic changes between treatments. While aboard the R/V Nathaniel B. Palmer during the CICLOPS research expedition, my fellow scientists along with the crew and Antarctic support staff were excellent colleagues and our collective work allowed for the results presented in Chapter 3. The fieldwork conducted across Antarctic seas over a roughly 2.5-month period was instrumental to my professional and personal development. This experience allowed me to take part in an international collaboration to study the coastal Antarctica region and experience the dynamic Southern Ocean firsthand. Being able to witness the complex, pristine, and thriving Antarctic biome inspired me in a fundamental way to continue studying the role of microbes in our biosphere. This experience also watered the seeds already present in me to expand my pursuit of helping to conserve, protect, and support Earth's natural habitats. From the Follows Lab, I learned how to code, 
develop numerical models, integrate my understand of the fundamentals of Ecology, and approach large-scale ecological questions from a molder's perspective, all of which were invaluable to designing this dissertation and identifying gaps in the literature that needed to be addressed first through lab and field work in order to best inform model development. Finally, the modeling study is in many ways the culmination of several goals defined when I started this dissertation - to learn numerical modeling methods, expand upon ecological models to include mutualistic interactions, and test hypotheses of how these interactions affect community dynamics and biogeochemical cycles. This dissertation is the start of future research on the impact of non-trophic interactions in marine microbial communities. I am grateful for these opportunities, access to cutting edge scientific resources, and the creative license to design and pursue this research with world class mentors, colleagues, and friends. 


\section{Bibliography}

A.-C. Alderkamp, M. M. Mills, G. L. van Dijken, P. Laan, C.-E. Thuróczy, L. J. Gerringa, H. J. de Baar, C. D. Payne, R. J. Visser, A. G. Buma, et al. Iron from melting glaciers fuels phytoplankton blooms in the Amundsen Sea (Southern Ocean): Phytoplankton characteristics and productivity. Deep Sea Research Part II: Topical Studies in Oceanography, 71:32-48, 2012.

S. Anders and W. Huber. Differential expression analysis for sequence count data. Nature Precedings, pages 1-1, 2010.

T. Anderson and P. l. B. Williams. Modelling the seasonal cycle of dissolved organic carbon at Station E1in the English Channel. Estuarine, Coastal and Shelf Science, 46(1):93-109, 1998.

A. L. Annett, M. Skiba, S. F. Henley, H. J. Venables, M. P. Meredith, P. J. Statham, and R. S. Ganeshram. Comparative roles of upwelling and glacial iron sources in Ryder Bay, coastal western Antarctic Peninsula. Marine Chemistry, 176:21-33, 2015.

A. L. Annett, J. N. Fitzsimmons, M. J. Séguret, M. Lagerström, M. P. Meredith, O. Schofield, and R. M. Sherrell. Controls on dissolved and particulate iron distributions in surface waters of the Western Antarctic Peninsula shelf. Marine Chemistry, 196:81-97, 2017.

M. Ardelan, O. Holm-Hansen, C. Hewes, C. Reiss, N. Silva, H. Dulaiova, E. Steinnes, and E. Sakshaug. Natural iron enrichment around the Antarctic Peninsula in the Southern Ocean. Biogeosciences Discussions, 6(4), 2009.

J. R. Aretakis, A. Gega, and J. M. Schrader. Absolute Measurements of mRNA Translation in Caulobacter crescentus Reveal Important Fitness Costs of Vitamin $\mathrm{B}_{12}$ Scavenging. MSystems, 4(4):e00170-19, 2019.

K. R. Arrigo and G. L. van Dijken. Annual changes in sea-ice, chlorophyll a, and primary production in the Ross Sea, Antarctica. Deep Sea Research Part II: Topical Studies in Oceanography, 51(1-3):117-138, 2004.

K. R. Arrigo, A. M. Weiss, and W. O. Smith Jr. Physical forcing of phytoplankton dynamics in the southwestern Ross Sea. Journal of Geophysical Research: Oceans, 103(C1):1007-1021, 1998a. 
K. R. Arrigo, D. Worthen, A. Schnell, and M. P. Lizotte. Primary production in Southern Ocean waters. Journal of Geophysical Research: Oceans, 103(C8): 15587-15600, 1998b.

K. R. Arrigo, D. H. Robinson, D. L. Worthen, R. B. Dunbar, G. R. DiTullio, M. VanWoert, and M. P. Lizotte. Phytoplankton community structure and the drawdown of nutrients and $\mathrm{CO}_{2}$ in the Southern Ocean. Science, 283(5400):365-367, 1999.

K. R. Arrigo, R. B. Dunbar, M. P. Lizotte, and D. Robinson. Taxon-specific differences in C:P and N:P drawdown for phytoplankton in the Ross Sea, Antarctica. Geophysical Research Letters, 29(19):44-1, 2002.

K. R. Arrigo, D. H. Robinson, R. B. Dunbar, A. R. Leventer, and M. P. Lizotte. Physical control of chlorophyll a, POC, and TPN distributions in the pack ice of the Ross Sea, Antarctica. Journal of Geophysical Research: Oceans, 108(C10), 2003.

K. R. Arrigo, G. van Dijken, and M. Long. Coastal Southern Ocean: A strong anthropogenic $\mathrm{CO}_{2}$ sink. Geophysical Research Letters, 35(21), 2008.

K. R. Arrigo, K. E. Lowry, and G. L. van Dijken. Annual changes in sea ice and phytoplankton in polynyas of the Amundsen Sea, Antarctica. Deep Sea Research Part II: Topical Studies in Oceanography, 71:5-15, 2012.

R. V. Banerjee and R. G. Matthews. Cobalamin-dependent methionine synthase. The FASEB journal, 4(5):1450-1459, 1990.

S. Becquevort, V. Rousseau, and C. Lancelot. Major and comparable roles for freeliving and attached bacteria in the degradation of Phaeocystis-derived organic matter in Belgian coastal waters of the North Sea. Aquatic Microbial Ecology, 14 (1):39-48, 1998.

S. Becquevort, C. Lancelot, and V. Schoemann. The role of iron in the bacterial degradation of organic matter derived from Phaeocystis antarctica. In Phaeocystis, major link in the biogeochemical cycling of climate-relevant elements, pages 119-135. Springer, 2007.

E. Bell. Life at extremes: environments, organisms, and strategies for survival, volume 1. Cabi, 2012.

S. J. Bender, D. M. Moran, M. R. McIlvin, H. Zheng, J. P. McCrow, J. Badger, G. R. DiTullio, A. E. Allen, and M. A. Saito. Colony formation in phaeocystis antarctica: connecting molecular mechanisms with iron biogeochemistry. Biogeosciences, 15 (16), 2018.

E. M. Bertrand, M. A. Saito, J. M. Rose, C. R. Riesselman, M. C. Lohan, A. E. Noble, P. A. Lee, and G. R. DiTullio. Vitamin $\mathrm{B}_{12}$ and iron colimitation of phytoplankton growth in the Ross Sea. Limnology and Oceanography, 52(3):1079-1093, 2007. 
E. M. Bertrand, M. A. Saito, Y. J. Jeon, and B. A. Neilan. Vitamin B 12 biosynthesis gene diversity in the ross sea: the identification of a new group of putative polar $\mathrm{B}_{12}$ biosynthesizers. Environmental microbiology, 13(5):1285-1298, 2011a.

E. M. Bertrand, M. A. Saito, P. A. Lee, R. B. Dunbar, P. N. Sedwick, and G. R. DiTullio. Iron limitation of a springtime bacterial and phytoplankton community in the Ross Sea: Implications for vitamin $\mathrm{B}_{12}$ nutrition. Frontiers in microbiology, 2 : $160,2011 b$.

E. M. Bertrand, A. E. Allen, C. L. Dupont, T. M. Norden-Krichmar, J. Bai, R. E. Valas, and M. A. Saito. Influence of cobalamin scarcity on diatom molecular physiology and identification of a cobalamin acquisition protein. Proceedings of the National Academy of Sciences, 109(26):E1762-E1771, 2012.

E. M. Bertrand, D. M. Moran, M. R. McIlvin, J. M. Hoffman, A. E. Allen, and M. A. Saito. Methionine synthase interreplacement in diatom cultures and communities: Implications for the persistence of $\mathrm{B}_{12}$ use by eukaryotic phytoplankton. Limnology and oceanography, 58(4):1431-1450, 2013.

E. M. Bertrand, J. P. McCrow, A. Moustafa, H. Zheng, J. B. McQuaid, T. O. Delmont, A. F. Post, R. E. Sipler, J. L. Spackeen, K. Xu, et al. Phytoplankton-bacterial interactions mediate micronutrient colimitation at the coastal Antarctic sea ice edge. Proceedings of the National Academy of Sciences, 112(32):9938-9943, 2015.

R. A. Bloodgood. Resorption of organelles containing microtubules. Cytobios, 9: 142-61, 031974.

S. Bonnet, E. A. Webb, C. Panzeca, D. M. Karl, D. G. Capone, and S. A. S. Wilhelmy. Vitamin $\mathrm{B}_{12}$ excretion by cultures of the marine cyanobacteria Crocosphaera and Synechococcus. Limnology and oceanography, 55(5):1959-1964, 2010.

I. Borovok, B. Gorovitz, R. Schreiber, Y. Aharonowitz, and G. Cohen. Coenzyme B 12 controls transcription of the Streptomyces class Ia ribonucleotide reductase nrdABS operon via a riboswitch mechanism. Journal of bacteriology, 188(7):2512-2520, 2006.

P. W. Boyd. The role of iron in the biogeochemistry of the southern ocean and equatorial pacific: a comparison of in situ iron enrichments. Deep Sea Research Part II: Topical Studies in Oceanography, 49(9-10):1803-1821, 2002.

E. Breton, C. Brunet, B. Sautour, and J.-M. Brylinski. Annual variations of phytoplankton biomass in the Eastern English Channel: comparison by pigment signatures and microscopic counts. Journal of Plankton Research, 22(8):1423-1440, 2000 .

B. Buchfink, C. Xie, and D. H. Huson. Fast and sensitive protein alignment using DIAMOND. Nature methods, 12(1):59, 2015. 
F. Bunbury, K. E. Helliwell, P. Mehrshahi, M. P. Davey, D. L. Salmon, A. Holzer, N. Smirnoff, and A. G. Smith. Physiological and molecular responses of a newly evolved auxotroph of Chlamydomonas to $\mathrm{B}_{12}$ deprivation. Plant Physiology, 2020.

C. Bunse and J. Pinhassi. Marine bacterioplankton seasonal succession dynamics. Trends in microbiology, 25(6):494-505, 2017.

N. J. Butterfield. Plankton ecology and the Proterozoic-Phanerozoic transition. Paleobiology, 23(2):247-262, 1997.

S. Capella-Gutiérrez, J. M. Silla-Martínez, and T. Gabaldón. trimAl: A tool for automated alignment trimming in large-scale phylogenetic analyses. Bioinformatics, 25(15):1972-1973, 2009.

A. Carlucci, S. Silbernagel, and P. McNally. Influence of temperature and solar radiation on persistence of vitamin $\mathrm{B}_{12}$, thiamine, and biotin in seawater. Journal of phycology, 5(4):302-305, 1969.

D. A. Caron, M. R. Dennett, D. J. Lonsdale, D. M. Moran, and L. Shalapyonok. Microzooplankton herbivory in the Ross sea, Antarctica. Deep Sea Research Part II: Topical Studies in Oceanography, 47(15-16):3249-3272, 2000.

M. H. Carr and K. Turekian. The geochemistry of cobalt. Geochimica et Cosmochimica Acta, 23(1-2):9-60, 1961.

P. Castagno, V. Capozzi, G. R. DiTullio, P. Falco, G. Fusco, S. R. Rintoul, G. Spezie, and G. Budillon. Rebound of shelf water salinity in the ross sea. Nature communications, 10(1):1-6, 2019.

S. W. Chisholm. Phytoplankton size. In Primary productivity and biogeochemical cycles in the sea, pages 213-237. Springer, 1992.

U. Christaki, D. Lefèvre, C. Georges, J. Colombet, P. Catala, C. Courties, T. SimeNgando, S. Blain, and I. Obernosterer. Microbial food web dynamics during spring phytoplankton blooms in the naturally iron-fertilized Kerguelen area (Southern Ocean). Biogeosciences, 11(23):6739, 2014.

M. J. Church, D. A. Hutchins, and H. W. Ducklow. Limitation of bacterial growth by dissolved organic matter and iron in the Southern Ocean. Appl. Environ. Microbiol., 66(2):455-466, 2000.

M. J. Church, E. F. DeLong, H. W. Ducklow, M. B. Karner, C. M. Preston, and D. M. Karl. Abundance and distribution of planktonic Archaea and Bacteria in the waters west of the Antarctic Peninsula. Limnology and Oceanography, 48(5):1893-1902, 2003 .

K. H. Coale, X. Wang, S. J. Tanner, and K. S. Johnson. Phytoplankton growth and biological response to iron and zinc addition in the Ross Sea and Antarctic Circumpolar Current along $170^{\circ} \mathrm{W}$. Deep Sea Research Part II: Topical Studies in Oceanography, 50(3-4):635-653, 2003. 
K. H. Coale, R. M. Gordon, and X. Wang. The distribution and behavior of dissolved and particulate iron and zinc in the Ross Sea and Antarctic circumpolar current along $170^{\circ}$ W. Deep Sea Research Part I: Oceanographic Research Papers, 52(2): 295-318, 2005.

N. R. Cohen, W. Gong, D. M. Moran, M. R. McIlvin, M. A. Saito, and A. Marchetti. Transcriptomic and proteomic responses of the oceanic diatom Pseudo-nitzschia granii to iron limitation. Environmental microbiology, 20(8):3109-3126, 2018.

M. T. Croft, A. D. Lawrence, E. Raux-Deery, M. J. Warren, and A. G. Smith. Algae acquire vitamin $\mathrm{B}_{12}$ through a symbiotic relationship with bacteria. Nature, 438 (7064):90-93, 2005.

M. T. Croft, M. J. Warren, and A. G. Smith. Algae need their vitamins. Eukaryotic cell, 5(8):1175-1183, 2006.

P. J. Crutzen. Ultraviolet on the increase. Nature, 356(6365):104-105, 1992.

N. Daugbjerg and L. Guillou. Phylogenetic analyses of Bolidophyceae (Heterokontophyta) using $r b c \mathrm{~L}$ gene sequences support their sister group relationship to diatoms. Phycologia, 40(2):153-161, 2001.

A. Davidson and H. Marchant. Binding of manganese by Antarctic Phaeocystis pouchetii and the role of bacteria in its release. Marine biology, 95(3):481-487, 1987.

H. J. De Baar, P. W. Boyd, K. H. Coale, M. R. Landry, A. Tsuda, P. Assmy, D. C. Bakker, Y. Bozec, R. T. Barber, M. A. Brzezinski, et al. Synthesis of iron fertilization experiments: from the iron age in the age of enlightenment. Journal of Geophysical Research: Oceans, 110(C9), 2005.

J. De Jong, V. Schoemann, D. Lannuzel, P. Croot, H. de Baar, and J.-L. Tison. Natural iron fertilization of the Atlantic sector of the Southern Ocean by continental shelf sources of the Antarctic Peninsula. Journal of Geophysical Research: Biogeosciences, 117(G1), 2012.

R. Death, J. Wadham, F. Monteiro, A. Le Brocq, M. Tranter, A. Ridgwell, S. Dutkiewicz, and R. Raiswell. Antarctic ice sheet fertilises the Southern Ocean. Biogeosciences, 11(10):2635-2643, 2014.

P. H. Degnan, M. E. Taga, and A. L. Goodman. Vitamin $\mathrm{B}_{12}$ as a modulator of gut microbial ecology. Cell metabolism, 20(5):769-778, 2014.

T. O. Delmont, K. M. Hammar, H. W. Ducklow, P. L. Yager, and A. F. Post. Phaeocystis antarctica blooms strongly influence bacterial community structures in the Amundsen Sea polynya. Frontiers in microbiology, 5:646, 2014.

T. O. Delmont, A. M. Eren, J. H. Vineis, and A. F. Post. Genome reconstructions indicate the partitioning of ecological functions inside a phytoplankton bloom in the Amundsen Sea, Antarctica. Frontiers in microbiology, 6:1090, 2015. 
D. Deobald, R. Hanna, S. Shahryari, G. Layer, and L. Adrian. Identification and characterization of a bacterial core methionine synthase. Scientific Reports, 10(1): $1-13,2020$.

S. L. Deppeler and A. T. Davidson. Southern Ocean phytoplankton in a changing climate. Frontiers in Marine Science, 4:40, 2017.

M. S. Dinniman, J. M. Klinck, and E. E. Hofmann. Sensitivity of circumpolar deep water transport and ice shelf basal melt along the West Antarctic Peninsula to changes in the winds. Journal of Climate, 25(14):4799-4816, 2012.

M. S. Dinniman, X. S. Asay-Davis, B. K. Galton-Fenzi, P. R. Holland, A. Jenkins, and R. Timmermann. Modeling ice shelf/ocean interaction in Antarctica: A review. Oceanography, 29(4):144-153, 2016. ISSN 10428275. doi: 10.5670/oceanog.2016.106.

G. DiTullio and M. E. Geesey. Photosynthetic pigments in marine algae and bacteria. Encyclopedia of environmental microbiology, 2003.

G. DiTullio, J. Grebmeier, K. Arrigo, M. Lizotte, D. Robinson, A. Leventer, J. Barry, M. VanWoert, and R. Dunbar. Rapid and early export of Phaeocystis antarctica blooms in the Ross Sea, Antarctica. Nature, 404(6778):595-598, 2000.

G. R. DiTullio and W. O. Smith. Spatial patterns in phytoplankton biomass and pigment distributions in the ross sea. Journal of Geophysical Research: Oceans, 101 (C8):18467-18477, 1996.

J. Dolfing. Syntrophy in microbial fuel cells. The ISME journal, 8(1):4-5, 2014.

A. C. Doxey, D. A. Kurtz, M. D. Lynch, L. A. Sauder, and J. D. Neufeld. Aquatic metagenomes implicate Thaumarchaeota in global cobalamin production. The ISME journal, 9(2):461-471, 2015.

C. L. Drennan, R. G. Matthews, and M. L. Ludwig. Cobalamin-dependent methionine synthase: the structure of a methylcobalamin-binding fragment and implications for other $\mathrm{B}_{12}$-dependent enzymes. Current opinion in structural biology, 4(6):919-929, 1994.

M. Droop. Auxotrophy and organic compounds in the nutrition of marine phytoplankton. Microbiology, 16(1):286-293, 1957.

M. Droop. Vitamins, phytoplankton and bacteria: symbiosis or scavenging? Journal of plankton research, 29(2):107-113, 2007.

H. Ducklow, C. Carlson, M. Church, D. Kirchman, D. Smith, and G. Steward. The seasonal development of the bacterioplankton bloom in the Ross Sea, Antarctica, 1994-1997. Deep Sea Research Part II: Topical Studies in Oceanography, 48(19-20): 4199-4221, 2001. 
H. W. Ducklow, W. Fraser, D. M. Karl, L. B. Quetin, R. M. Ross, R. C. Smith, S. E. Stammerjohn, M. Vernet, and R. M. Daniels. Water-column processes in the West Antarctic Peninsula and the Ross Sea: interannual variations and foodweb structure. Deep Sea Research Part II: Topical Studies in Oceanography, 53(8-10): 834-852, 2006.

H. W. Ducklow, K. Baker, D. G. Martinson, L. B. Quetin, R. M. Ross, R. C. Smith, S. E. Stammerjohn, M. Vernet, and W. Fraser. Marine pelagic ecosystems: the west Antarctic Peninsula. Philosophical Transactions of the Royal Society B: Biological Sciences, 362(1477):67-94, 2007.

R. B. Dunbar, K. R. Arrigo, M. Lutz, G. R. Ditullio, A. R. Leventer, M. P. Lizotte, M. P. Van Woert, and D. H. Robinson. Non-Redfield production and export of marine organic matter: A recurrent part of the annual cycle in the Ross Sea, Antarctica. Biogeochemistry of the Ross Sea, 78:179-196, 2003.

S. Dutkiewicz, P. Cermeno, O. Jahn, M. J. Follows, A. E. Hickman, D. A. Taniguchi, and B. A. Ward. Dimensions of marine phytoplankton diversity. Biogeosciences, 17 (3):609-634, 2020.

K. A. Ellis, N. R. Cohen, C. Moreno, and A. Marchetti. Cobalamin-independent methionine synthase distribution and influence on vitamin $\mathrm{B}_{12}$ growth requirements in marine diatoms. Protist, 168(1):32-47, 2017.

J. T. M. Elzenga, H. B. Prins, and J. Stefels. The role of extracellular carbonic anhydrase activity in inorganic carbon utilization of Phaeocystis globosa (Prymnesiophyceae): A comparison with other marine algae using the isotopic disequilibrium technique. Limnology and Oceanography, 45(2):372-380, 2000.

A. Eschenmoser. Vitamin $\mathrm{B}_{12}$ : experiments concerning the origin of its molecular structure. Angewandte Chemie International Edition in English, 27(1):5-39, 1988.

C. B. Field, M. J. Behrenfeld, J. T. Randerson, and P. Falkowski. Primary production of the biosphere: integrating terrestrial and oceanic components. science, 281(5374): 237-240, 1998.

Z. V. Finkel, J. Beardall, K. J. Flynn, A. Quigg, T. A. V. Rees, and J. A. Raven. Phytoplankton in a changing world: cell size and elemental stoichiometry. Journal of plankton research, 32(1):119-137, 2010.

S. Fitzwater, K. Johnson, R. Gordon, K. Coale, and W. Smith Jr. Trace metal concentrations in the ross sea and their relationship with nutrients and phytoplankton growth. Deep Sea Research Part II: Topical Studies in Oceanography, 47(15-16):3159-3179, 2000.

N. S. Garcia, P. N. Sedwick, and G. R. DiTullio. Influence of irradiance and iron on the growth of colonial Phaeocystis antarctica: implications for seasonal bloom dynamics in the Ross Sea, Antarctica. Aquatic microbial ecology, 57(2):203-220, 2009. 
L. J. Gerringa, A.-C. Alderkamp, P. Laan, C.-E. Thuroczy, H. J. De Baar, M. M. Mills, G. L. van Dijken, H. van Haren, and K. R. Arrigo. Iron from melting glaciers fuels the phytoplankton blooms in Amundsen Sea (Southern Ocean): Iron biogeochemistry. Deep Sea Research Part II: Topical Studies in Oceanography, 71: $16-31,2012$.

J.-F. Ghiglione, P. E. Galand, T. Pommier, C. Pedrós-Alió, E. W. Maas, K. Bakker, S. Bertilson, D. L. Kirchman, C. Lovejoy, P. L. Yager, et al. Pole-to-pole biogeography of surface and deep marine bacterial communities. Proceedings of the National Academy of Sciences, 109(43):17633-17638, 2012.

S. J. Giovannoni. Vitamins in the sea. Proceedings of the National Academy of Sciences, 109(35):13888-13889, 2012.

C. J. Gobler, C. Norman, C. Panzeca, G. T. Taylor, and S. A. Sañudo-Wilhelmy. Effect of B-vitamins $\left(\mathrm{B}_{1}, \mathrm{~B}_{12}\right)$ and inorganic nutrients on algal bloom dynamics in a coastal ecosystem. Aquatic Microbial Ecology, 49(2):181-194, 2007.

A. Goffart, G. Catalano, and J.-H. Hecq. Factors controlling the distribution of diatoms and Phaeocystis in the Ross Sea. Journal of Marine Systems, 27(1-3): 161-175, 2000.

L. Gómez-Consarnau, R. Sachdeva, S. M. Gifford, L. S. Cutter, J. A. Fuhrman, S. A. Sañudo-Wilhelmy, and M. A. Moran. Mosaic patterns of B-vitamin synthesis and utilization in a natural marine microbial community. Environmental microbiology, 20(8):2809-2823, 2018.

J. C. Gonzalez, R. V. Banerjee, S. Huang, J. S. Sumner, and R. G. Matthews. Comparison of cobalamin-independent and cobalamin-dependent methionine synthases from escherichia coli: two solutions to the same chemical problem. Biochemistry, 31(26):6045-6056, 1992.

M. A. Grant, E. Kazamia, P. Cicuta, and A. G. Smith. Direct exchange of vitamin $\mathrm{B}_{12}$ is demonstrated by modelling the growth dynamics of algal-bacterial cocultures. The ISME journal, 8(7):1418-1427, 2014.

R. C. Greene. Biosynthesis of methionine. Escherichia coli and Salmonella typhimurium: Cellular and Molecular Biology, pages 542-560, 1996.

J. P. Grover. Resource competition in a variable environment: phytoplankton growing according to Monod's model. American Naturalist, pages 771-789, 1990.

J. P. Grover. Resource competition in a variable environment: phytoplankton growing according to the variable-internal-stores model. The American Naturalist, 138(4): 811-835, 1991.

M. Grube, T. Cernava, J. Soh, S. Fuchs, I. Aschenbrenner, C. Lassek, U. Wegner, D. Becher, K. Riedel, C. W. Sensen, et al. Exploring functional contexts of symbiotic 
sustain within lichen-associated bacteria by comparative omics. The ISME journal, $9(2): 412-424,2015$.

N. J. Hawco and M. A. Saito. Competitive inhibition of cobalt uptake by zinc and manganese in a pacific Prochlorococcus strain: Insights into metal homeostasis in a streamlined oligotrophic cyanobacterium. Limnology and Oceanography, 63(5): 2229-2249, 2018.

J. R. Hawkings, J. L. Wadham, M. Tranter, R. Raiswell, L. G. Benning, P. J. Statham, A. Tedstone, P. Nienow, K. Lee, and J. Telling. Ice sheets as a significant source of highly reactive nanoparticulate iron to the oceans. Nature communications, 5(1): $1-8,2014$.

J. R. Hawkings, L. G. Benning, R. Raiswell, B. Kaulich, T. Araki, M. Abyaneh, A. Stockdale, M. Koch-Müller, J. L. Wadham, and M. Tranter. Biolabile ferrous iron bearing nanoparticles in glacial sediments. Earth and Planetary Science Letters, 493:92-101, 2018.

R. M. Hazen, G. Hystad, J. J. Golden, D. R. Hummer, C. Liu, R. T. Downs, S. M. Morrison, J. Ralph, and E. S. Grew. Cobalt mineral ecology. American Mineralogist, 102(1):108-116, 2017.

K. R. Heal, L. T. Carlson, A. H. Devol, E. V. Armbrust, J. W. Moffett, D. A. Stahl, and A. E. Ingalls. Determination of four forms of vitamin $\mathrm{B}_{12}$ and other B vitamins in seawater by liquid chromatography/tandem mass spectrometry. Rapid Communications in Mass Spectrometry, 28(22):2398-2404, 2014.

K. R. Heal, W. Qin, F. Ribalet, A. D. Bertagnolli, W. Coyote-Maestas, L. R. Hmelo, J. W. Moffett, A. H. Devol, E. V. Armbrust, D. A. Stahl, et al. Two distinct pools of $\mathrm{B}_{12}$ analogs reveal community interdependencies in the ocean. Proceedings of the National Academy of Sciences, 114(2):364-369, 2017.

K. E. Helliwell. The roles of B vitamins in phytoplankton nutrition: new perspectives and prospects. New Phytologist, 216(1):62-68, 2017.

K. E. Helliwell, G. L. Wheeler, K. C. Leptos, R. E. Goldstein, and A. G. Smith. Insights into the evolution of vitamin $\mathrm{B}_{12}$ auxotrophy from sequenced algal genomes. Molecular biology and evolution, 28(10):2921-2933, 2011.

K. E. Helliwell, G. L. Wheeler, and A. G. Smith. Widespread decay of vitamin-related pathways: coincidence or consequence? Trends in Genetics, 29(8):469-478, 2013.

K. E. Helliwell, M. A. Scaife, S. Sasso, A. P. U. Araujo, S. Purton, and A. G. Smith. Unraveling vitamin $\mathrm{B}_{12}$-responsive gene regulation in algae. Plant physiology, 165 (1):388-397, 2014.

K. E. Helliwell, S. Collins, E. Kazamia, S. Purton, G. L. Wheeler, and A. G. Smith. Fundamental shift in vitamin $\mathrm{B}_{12}$ eco-physiology of a model alga demonstrated by experimental evolution. The ISME journal, 9(6):1446-1455, 2015. 
K. E. Helliwell, A. D. Lawrence, A. Holzer, U. J. Kudahl, S. Sasso, B. Kräutler, D. J. Scanlan, M. J. Warren, and A. G. Smith. Cyanobacteria and eukaryotic algae use different chemical variants of vitamin $\mathrm{B}_{12}$. Current Biology, 26(8):999-1008, 2016.

T.-Y. Ho, A. Quigg, Z. V. Finkel, A. J. Milligan, K. Wyman, P. G. Falkowski, and F. M. Morel. The elemental composition of some marine phytoplankton. Journal of phycology, 39(6):1145-1159, 2003.

A. Hodson, A. Nowak, M. Sabacka, A. Jungblut, F. Navarro, D. Pearce, M. L. ÁvilaJiménez, P. Convey, and G. Vieira. Climatically sensitive transfer of iron to maritime Antarctic ecosystems by surface runoff. Nature communications, 8(1):1-7, 2017.

C. S. Hughes, S. Foehr, D. A. Garfield, E. E. Furlong, L. M. Steinmetz, and J. Krijgsveld. Ultrasensitive proteome analysis using paramagnetic bead technology. Molecular systems biology, 10(10), 2014.

A. Ito and J. F. Kok. Do dust emissions from sparsely vegetated regions dominate atmospheric iron supply to the Southern Ocean? Journal of Geophysical Research: Atmospheres, 122(7):3987-4002, 2017.

A. Ito, S. Myriokefalitakis, M. Kanakidou, N. M. Mahowald, R. A. Scanza, D. S. Hamilton, A. R. Baker, T. Jickells, M. Sarin, S. Bikkina, et al. Pyrogenic iron: The missing link to high iron solubility in aerosols. Science advances, 5(5):eaau7671, 2019.

D. R. Jackett and T. J. McDougall. A neutral density variable for the world's oceans. Journal of Physical Oceanography, 27(2):237-263, 1997.

I. Janse, M. Van Rijssel, J. C. Gottschal, C. Lancelot, and W. W. Gieskes. Carbohydrates in the North Sea during spring blooms of Phaeocystis: a specific fingerprint. Aquatic microbial ecology, 10(1):97-103, 1996.

R. J. Kadner and K. J. Heller. Mutual inhibition of cobalamin and siderophore uptake systems suggests their competition for TonB function. Journal of Bacteriology, 177 (17):4829-4835, 1995.

K. M. Kalanetra, N. Bano, and J. T. Hollibaugh. Ammonia-oxidizing Archaea in the Arctic Ocean and Antarctic coastal waters. Environmental microbiology, 11(9): 2434-2445, 2009.

K. Katoh and D. M. Standley. MAFFT multiple sequence alignment software version 7: improvements in performance and usability. Molecular biology and evolution, 30 (4):772-780, 2013.

D. E. Kaufman. Using High-Resolution Glider Data and Biogeochemical Modeling to Investigate Phytoplankton Variability in the Ross Sea. PhD thesis, College of William and Mary, 2017. 
E. Kazamia, H. Czesnick, T. T. V. Nguyen, M. T. Croft, E. Sherwood, S. Sasso, S. J. Hodson, M. J. Warren, and A. G. Smith. Mutualistic interactions between vitamin $\mathrm{B}_{12}$-dependent algae and heterotrophic bacteria exhibit regulation. Environmental microbiology, 14(6):1466-1476, 2012.

M. D. Kazanov, A. G. Vitreschak, and M. S. Gelfand. Abundance and functional diversity of riboswitches in microbial communities. BMC genomics, 8(1):347, 2007.

D. L. Kirchman, B. Meon, M. T. Cottrell, D. A. Hutchins, D. Weeks, and K. W. Bruland. Carbon versus iron limitation of bacterial growth in the California upwelling regime. Limnology and Oceanography, 45(8):1681-1688, 2000.

P. E. Klebba, J. M. Rutz, J. Liu, and C. K. Murphy. Mechanisms of TonB-catalyzed iron transport through the enteric bacterial cell envelope. Journal of bioenergetics and biomembranes, 25(6):603-611, 1993.

F. Koch, T. K. Hattenrath-Lehmann, J. A. Goleski, S. Sañudo-Wilhelmy, N. S. Fisher, and C. J. Gobler. Vitamin B1 and $\mathrm{B}_{12}$ uptake and cycling by plankton communities in coastal ecosystems. Frontiers in microbiology, 3:363, 2012.

A. E. Koid, Z. Liu, R. Terrado, A. C. Jones, D. A. Caron, and K. B. Heidelberg. Comparative transcriptome analysis of four prymnesiophyte algae. PLoS One, 9(6), 2014.

B. Kräutler. Vitamin $\mathrm{B}_{12}$ : Chemistry and biochemistry, 2005.

V. Kurvari, F. Qian, and W. J. Snell. Increased transcript levels of a methionine synthase during adhesion-induced activation of Chlamydomonas reinhardtiigametes. Plant molecular biology, 29(6):1235-1252, 1995.

C. Lancelot, A. de Montety, H. Goosse, S. Becquevort, V. Schoemann, B. Pasquer, and M. Vancoppenolle. Spatial distribution of the iron supply to phytoplankton in the Southern Ocean: a model study. Biogeosciences, 6(12):2861-2878, 2009.

D. Lannuzel, V. Schoemann, J. De Jong, B. Pasquer, P. Van der Merwe, F. Masson, J.-L. Tison, and A. Bowie. Distribution of dissolved iron in Antarctic sea ice: Spatial, seasonal, and inter-annual variability. Journal of Geophysical Research: Biogeosciences, 115(G3), 2010.

D. Lannuzel, M. Vancoppenolle, P. Van der Merwe, J. De Jong, K. M. Meiners, M. Grotti, J. Nishioka, and V. Schoemann. Iron in sea ice: Review and new insights. Elem Sci Anth, 4, 2016.

C. Laufkötter, A. A. Stern, J. G. John, C. A. Stock, and J. P. Dunne. Glacial iron sources stimulate the southern ocean carbon cycle. Geophysical Research Letters, 45(24):13-377, 2018. 
A. D. Lawrence, E. Nemoto-Smith, E. Deery, J. A. Baker, S. Schroeder, D. G. Brown, J. M. Tullet, M. J. Howard, I. R. Brown, A. G. Smith, et al. Construction of fluorescent analogs to follow the uptake and distribution of cobalamin (vitamin $\mathrm{B}_{12}$ ) in bacteria, worms, and plants. Cell chemical biology, 25(8):941-951, 2018.

A. Leventer and R. B. Dunbar. Factors influencing the distribution of diatoms and other algae in the Ross Sea. Journal of Geophysical Research: Oceans, 101(C8): 18489-18500, 1996.

E. Litchman, C. A. Klausmeier, O. M. Schofield, and P. G. Falkowski. The role of functional traits and trade-offs in structuring phytoplankton communities: scaling from cellular to ecosystem level. Ecology letters, 10(12):1170-1181, 2007.

K. Lochte, P. K. Bjørnsen, H. Giesenhagen, and A. Weber. Bacterial standing stock and production and their relation to phytoplankton in the Southern Ocean. Deep Sea Research Part II: Topical Studies in Oceanography, 44(1-2):321-340, 1997.

E. Marañón. Cell size as a key determinant of phytoplankton metabolism and community structure. Annual Review of Marine Science, 7(1):241-264, 2015. doi: 10.1146/annurev-marine-010814-015955. PMID: 25062405.

M. Mars Brisbin and S. Mitarai. Differential gene expression supports a resourceintensive, defensive role for colony production in the bloom-forming haptophyte, Phaeocystis globosa. Journal of Eukaryotic Microbiology, 66(5):788-801, 2019.

J.-H. Martens, H. Barg, M. Warren, and D. Jahn. Microbial production of vitamin $\mathrm{B}_{12}$. Applied microbiology and biotechnology, 58(3):275-285, 2002.

J. H. Martin. Glacial-interglacial $\mathrm{CO}_{2}$ change: The iron hypothesis. Paleoceanography, $5(1): 1-13,1990$.

S. Mathot, W. O. Smith Jr, C. A. Carlson, D. L. Garrison, M. M. Gowing, and C. L. Vickers. Carbon partitioning within Phaeocystis antarctica (Prymnesiophyceae) colonies in the Ross Sea, Antarctica. Journal of Phycology, 36(6):1049-1056, 2000.

E. Mattson, A. Karlsson, W. O. Smith, and K. Abrahamsson. The relationship between biophysical variables and halocarbon distributions in the waters of the Amundsen and Ross Seas, Antarctica. Marine Chemistry, 140-141(August):1-9, 2012. ISSN 03044203. doi: 10.1016/j.marchem.2012.07.002.

J. P. McCutcheon, B. R. McDonald, and N. A. Moran. Convergent evolution of metabolic roles in bacterial co-symbionts of insects. Proceedings of the National Academy of Sciences, 106(36):15394-15399, 2009.

D. J. McGillicuddy, P. N. Sedwick, M. S. Dinniman, K. R. Arrigo, T. S. Bibby, B. J. W. Greenan, E. E. Hofmann, J. M. Klinck, W. O. Smith, S. L. Mack, and et al. Iron supply and demand in an Antarctic shelf ecosystem. Geophysical Research Letters, 42(19):8088-8097, Aug 2015. doi: 10.1002/2015gl065727. 
M. T. Mee, J. J. Collins, G. M. Church, and H. H. Wang. Syntrophic exchange in synthetic microbial communities. Proceedings of the National Academy of Sciences, 111(20):E2149-E2156, 2014.

M. M. Mills, A.-C. Alderkamp, C.-E. Thuróczy, G. L. van Dijken, P. Laan, H. J. de Baar, and K. R. Arrigo. Phytoplankton biomass and pigment responses to Fe amendments in the Pine Island and Amundsen polynyas. Deep Sea Research Part II: Topical Studies in Oceanography, 71:61-76, 2012.

B. G. Mitchell and O. Holm-Hansen. Observations of modeling of the Antartic phytoplankton crop in relation to mixing depth. Deep Sea Research Part A. Oceanographic Research Papers, 38(8-9):981-1007, 1991.

B. G. Mitchell, E. A. Brody, O. Holm-Hansen, C. McClain, and J. Bishop. Light limitation of phytoplankton biomass and macronutrient utilization in the Southern Ocean. Limnology and Oceanography, 36(8):1662-1677, 1991.

J. W. Moffett and J. Ho. Oxidation of cobalt and manganese in seawater via a common microbially catalyzed pathway. Geochimica et Cosmochimica Acta, 60(18): 3415-3424, 1996.

M. Mongin, D. M. Nelson, P. Pondaven, and P. Tréguer. Simulation of upper-ocean biogeochemistry with a flexible-composition phytoplankton model: $\mathrm{C}, \mathrm{N}$ and $\mathrm{Si}$ cycling and Fe limitation in the Southern Ocean. Deep Sea Research Part II: Topical Studies in Oceanography, 53(5-7):601-619, 2006.

C. Moore, M. Mills, K. Arrigo, I. Berman-Frank, L. Bopp, P. Boyd, E. Galbraith, R. Geider, C. Guieu, S. Jaccard, et al. Processes and patterns of oceanic nutrient limitation. Nature geoscience, 6(9):701-710, 2013.

J. J. Morris, R. E. Lenski, and E. R. Zinser. The Black Queen Hypothesis: evolution of dependencies through adaptive gene loss. MBio, 3(2):e00036-12, 2012.

N. R. Movva, K. Nakamura, and M. Inouye. Gene structure of the OmpA protein, a major surface protein of Escherichia coli required for cell-cell interaction. Journal of molecular biology, 143(3):317-328, 1980.

A. Murray, C. Preston, R. Massana, L. Taylor, A. Blakis, K. Wu, and E. DeLong. Seasonal and spatial variability of bacterial and archaeal assemblages in the coastal waters near Anvers Island, Antarctica. Appl. Environ. Microbiol., 64(7):2585-2595, 1998.

K. Muylaert, R. Gonzales, M. Franck, M. Lionard, C. Van der Zee, A. Cattrijsse, K. Sabbe, L. Chou, and W. Vyverman. Spatial variation in phytoplankton dynamics in the Belgian coastal zone of the North Sea studied by microscopy, HPLCCHEMTAX and underway fluorescence recordings. Journal of Sea Research, 55(4): 253-265, 2006. 
Y. Nakayama, R. Timmermann, C. B. Rodehacke, M. Schröder, and H. H. Hellmer. Modeling the spreading of glacial meltwater from the Amundsen and Bellingshausen Seas. Geophysical Research Letters, 41(22):7942-7949, 2014.

C. Nef, S. Jung, F. Mairet, R. Kaas, D. Grizeau, and M. Garnier. How haptophytes microalgae mitigate vitamin $\mathrm{B}_{12}$ limitation. Scientific reports, 9(1):1-11, 2019.

J. C. Nejstgaard, K. W. Tang, M. Steinke, J. Dutz, M. Koski, E. Antajan, and J. D. Long. Zooplankton grazing on Phaeocystis: a quantitative review and future challenges. Biogeochemistry, 83(1-3):147-172, 2007.

A. I. Nesvizhskii. Proteogenomics: concepts, applications and computational strategies. Nature methods, 11(11):1114, 2014.

E. Newman, L. Budman, E. Chan, R. Greene, R. Lin, C. Woldringh, and R. DâĂŹAri. Lack of S-adenosylmethionine results in a cell division defect in Escherichia coli. Journal of bacteriology, 180(14):3614-3619, 1998.

L.-T. Nguyen, H. A. Schmidt, A. Von Haeseler, and B. Q. Minh. IQ-TREE: A fast and effective stochastic algorithm for estimating maximum-likelihood phylogenies. Molecular biology and evolution, 32(1):268-274, 2015.

A. E. Noble, D. M. Moran, A. E. Allen, and M. a. Saito. Dissolved and particulate trace metal micronutrients under the McMurdo Sound seasonal sea ice: basal sea ice communities as a capacitor for iron. Frontiers in chemistry, 1(October):25, 2013. ISSN 2296-2646. doi: 10.3389/fchem.2013.00025.

M. Oborník and B. R. Green. Mosaic origin of the heme biosynthesis pathway in photosynthetic eukaryotes. Molecular biology and evolution, 22(12):2343-2353, 2005.

H. Oliver, P. St-Laurent, R. M. Sherrell, and P. L. Yager. Modeling iron and light controls on the summer Phaeocystis antarctica bloom in the Amundsen Sea Polynya. Global Biogeochemical Cycles, 33(5):570-596, 2019.

A. H. Orsi and C. L. Wiederwohl. A recount of Ross Sea waters. Deep Sea Research Part II: Topical Studies in Oceanography, 56(13-14):778-795, 2009.

J. D. Pakulski, R. B. Coffin, C. A. Kelley, S. L. Holder, R. Downer, P. Aas, M. M. Lyons, and W. H. Jeffrey. Iron stimulation of antarctic bacteria. Nature, 383(6596): 133-134, 1996.

C. Panzeca, A. Tovar-Sanchez, S. Agustí, I. Reche, C. M. Duarte, G. T. Taylor, and S. A. Sañudo-Wilhelmy. B vitamins as regulators of phytoplankton dynamics. Eos, Transactions American Geophysical Union, 87(52):593-596, 2006.

C. Panzeca, A. J. Beck, K. Leblanc, G. Taylor, D. Hutchins, and S. Sañudo-Wilhelmy. Potential cobalt limitation of vitamin $\mathrm{B}_{12}$ synthesis in the North Atlantic Ocean. Global biogeochemical cycles, 22(2), 2008. 
C. Panzeca, A. J. Beck, A. Tovar-Sanchez, J. Segovia-Zavala, G. T. Taylor, C. J. Gobler, and S. A. Sanudo-Wilhelmy. Distributions of dissolved vitamin $\mathrm{B}_{12}$ and Co in coastal and open-ocean environments. Estuarine, Coastal and Shelf Science, 85 (2):223-230, 2009.

N. Pavelka, M. Pelizzola, C. Vizzardelli, M. Capozzoli, A. Splendiani, F. Granucci, and P. Ricciardi-Castagnoli. A power law global error model for the identification of differentially expressed genes in microarray data. BMC bioinformatics, 5(1):203, 2004.

N. Pavelka, M. L. Fournier, S. K. Swanson, M. Pelizzola, P. Ricciardi-Castagnoli, L. Florens, and M. P. Washburn. Statistical similarities between transcriptomics and quantitative shotgun proteomics data. Molecular \& Cellular Proteomics, 7(4): 631-644, 2008.

G. J. Pazour, N. Agrin, J. Leszyk, and G. B. Witman. Proteomic analysis of a eukaryotic cilium. The Journal of cell biology, 170(1):103-113, 2005.

K. Petrou, S. A. Kranz, S. Trimborn, C. S. Hassler, S. B. Ameijeiras, O. Sackett, P. J. Ralph, and A. T. Davidson. Southern ocean phytoplankton physiology in a changing climate. Journal of plant physiology, 203:135-150, 2016.

H. Planquette, R. M. Sherrell, S. Stammerjohn, and M. P. Field. Particulate iron delivery to the water column of the Amundsen Sea, Antarctica. Marine Chemistry, 153:15-30, 2013.

H. Ploug, W. Stolte, E. H. G. Epping, and B. B. Jørgensen. Diffusive boundary layers and growth in Phaeocystis colonies-a microelectrode study. In 5th European Marine Microbiology Symposium. (5th EMMS) BergenAugust., volume 11, 1996.

H. Ploug, W. Stolte, and B. B. Jørgensen. Diffusive boundary layers of the colonyforming plankton alga phaeocystis sp.: implications for nutrient uptake and cellular growth. Limnology and Oceanography, 44(8):1959-1967, 1999.

E. Popova, R. Pollard, M. Lucas, H. Venables, and T. Anderson. Real-time forecasting of ecosystem dynamics during the CROZEX experiment and the roles of light, iron, silicate, and circulation. Deep Sea Research Part II: Topical Studies in Oceanography, 54(18-20):1966-1988, 2007.

N. Price and F. Morel. Cadmium and cobalt substitution for zinc in a marine diatom. Nature, 344(6267):658-660, 1990.

M. Putt, G. Miceli, and D. K. Stoecker. Association of bacteria with Phaeocystis sp. in McMurdo Sound, Antarctica. Marine Ecology Progress Series, 105:179-179, 1994.

R. Raiswell, J. R. Hawkings, L. G. Benning, A. R. Baker, R. Death, S. Albani, N. Mahowald, M. D. Krom, S. W. Poulton, J. Wadham, et al. Potentially bioavailable iron delivery by iceberg-hosted sediments and atmospheric dust to the polar oceans. Biogeosciences, 13(13):3887-3900, 2016. 
E. Raux, H. Schubert, and M. Warren. Biosynthesis of cobalamin (vitamin $\mathrm{B}_{12}$ ): a bacterial conundrum. Cellular and Molecular Life Sciences CMLS, 57(13-14): 1880-1893, 2000.

E. Rignot, S. Jacobs, J. Mouginot, and B. Scheuchl. Ice-shelf melting around Antarctica. Science, 341(6143):266-270, 2013.

D. A. Rodionov, A. G. Vitreschak, A. A. Mironov, and M. S. Gelfand. Comparative genomics of the vitamin $\mathrm{B}_{12}$ metabolism and regulation in prokaryotes. Journal of Biological Chemistry, 278(42):41148-41159, 2003.

M. F. Romine, D. A. Rodionov, Y. Maezato, L. N. Anderson, P. Nandhikonda, I. A. Rodionova, A. Carre, X. Li, C. Xu, T. R. Clauss, et al. Elucidation of roles for vitamin $\mathrm{B}_{12}$ in regulation of folate, ubiquinone, and methionine metabolism. Proceedings of the National Academy of Sciences, 114(7):E1205-E1214, 2017.

J. R. Roth, J. Lawrence, and T. Bobik. Cobalamin (coenzyme $\mathrm{B}_{12}$ ): synthesis and biological significance. Annual review of microbiology, 50(1):137-181, 1996.

V. Rousseau, D. Vaulot, R. Casotti, V. Cariou, J. Lenz, J. Gunkel, and M. Baumann. The life cycle of phaeocystis (prymnesiophycaea): evidence and hypotheses. Journal of marine systems, 5(1):23-39, 1994.

V. Rousseau, M.-J. Chrétiennot-Dinet, A. Jacobsen, P. Verity, and S. Whipple. The life cycle of Phaeocystis: state of knowledge and presumptive role in ecology. Biogeochemistry, 83(1-3):29-47, 2007.

M. A. Saito and T. J. Goepfert. Zinc-cobalt colimitation of Phaeocystis antarctica. Limnology and Oceanography, 53(1):266-275, 2008.

M. A. Saito and J. W. Moffett. Complexation of cobalt by natural organic ligands in the Sargasso Sea as determined by a new high-sensitivity electrochemical cobalt speciation method suitable for open ocean work. Marine chemistry, 75(1-2):49-68, 2001.

M. A. Saito, J. W. Moffett, S. W. Chisholm, and J. B. Waterbury. Cobalt limitation and uptake in Prochlorococcus. Limnology and Oceanography, 47(6):1629-1636, 2002.

M. A. Saito, J. W. Moffett, and G. R. DiTullio. Cobalt and nickel in the peru upwelling region: A major flux of labile cobalt utilized as a micronutrient. Global Biogeochemical Cycles, 18(4), 2004.

M. A. Saito, T. J. Goepfert, A. E. Noble, E. M. Bertrand, P. N. Sedwick, and G. R. DiTullio. A seasonal study of dissolved cobalt in the Ross Sea, Antarctica: Micronutrient behavior, absence of scavenging, and relationships with $\mathrm{Zd}, \mathrm{Cd}$, and P. Biogeosciences, 7(12), 2010. 
M. A. Saito, M. R. McIlvin, D. M. Moran, A. E. Santoro, C. L. Dupont, P. A. Rafter, J. K. Saunders, D. Kaul, C. H. Lamborg, M. Westley, et al. Abundant nitriteoxidizing metalloenzymes in the mesopelagic zone of the tropical Pacific Ocean. Nature Geoscience, pages 1-8, 2020.

S. Sañudo-Wilhelmy, C. Gobler, M. Okbamichael, and G. Taylor. Regulation of phytoplankton dynamics by vitamin $\mathrm{B}_{12}$. Geophysical research letters, 33(4), 2006.

S. A. Sañudo-Wilhelmy, L. S. Cutter, R. Durazo, E. A. Smail, L. Gómez-Consarnau, E. A. Webb, M. G. Prokopenko, W. M. Berelson, and D. M. Karl. Multiple Bvitamin depletion in large areas of the coastal ocean. Proceedings of the National Academy of Sciences, 109(35):14041-14045, 2012.

M. J. Schneider, M. Ulland, and R. D. Sloboda. A protein methylation pathway in Chlamydomonas flagella is active during flagellar resorption. Molecular biology of the cell, 19(10):4319-4327, 2008.

V. Schoemann, R. Wollast, L. Chou, and C. Lancelot. Effects of photosynthesis on the accumulation of $\mathrm{Mn}$ and Fe by Phaeocystis colonies. Limnology and Oceanography, 46(5):1065-1076, 2001.

V. Schoemann, S. Becquevort, J. Stefels, V. Rousseau, and C. Lancelot. Phaeocystis blooms in the global ocean and their controlling mechanisms: A review. Journal of Sea Research, 53(1-2):43-66, 2005.

P. Sedwick, N. Garcia, S. Riseman, C. Marsay, and G. DiTullio. Evidence for high iron requirements of colonial Phaeocystis antarctica at low irradiance. In Phaeocystis, major link in the biogeochemical cycling of climate-relevant elements, pages 83-97. Springer, 2007.

P. N. Sedwick and G. R. DiTullio. Regulation of algal blooms in Antarctic shelf waters by the release of iron from melting sea ice. Geophysical Research Letters, 24(20): 2515-2518, 1997.

P. N. Sedwick, G. R. DiTullio, and D. J. Mackey. Iron and manganese in the ross sea, antarctica: seasonal iron limitation in antarctic shelf waters. Journal of Geophysical Research: Oceans, 105(C5):11321-11336, 2000.

E. C. Seth and M. E. Taga. Nutrient cross-feeding in the microbial world. Frontiers in microbiology, 5:350, 2014.

T. J. Shaw, R. Raiswell, C. Hexel, H. Vu, W. Moore, R. Dudgeon, and K. Smith Jr. Input, composition, and potential impact of terrigenous material from free-drifting icebergs in the Weddell Sea. Deep Sea Research Part II: Topical Studies in Oceanography, 58(11-12):1376-1383, 2011.

A. N. Shelton, E. C. Seth, K. C. Mok, A. W. Han, S. N. Jackson, D. R. Haft, and M. E. Taga. Uneven distribution of cobamide biosynthesis and dependence in bacteria predicted by comparative genomics. The ISME journal, 13(3):789-804, 2019. 
R. Sherrell, M. Lagerström, K. Forsch, S. Stammerjohn, and P. Yager. Dynamics of dissolved iron and other bioactive trace metals ( $\mathrm{Mn}, \mathrm{Ni}, \mathrm{Cu}, \mathrm{Zn}$ ) in the Amundsen Sea Polynya, Antarctica. Elem Sci Anth, 3, 2015.

R. M. Sherrell, P. L. Yager, P. St-Laurent, M. S. Dinniman, S. E. Stammerjohn, M. Lagerstrom, and K. M. Harazin. High iron in outflow waters from the Dotson Ice Shelf cavity, Amundsen Sea, West Antarctica: is glacial meltwater really the source? In 2018 Ocean Sciences Meeting. AGU, 2018.

W. Shou, S. Ram, and J. M. Vilar. Synthetic cooperation in engineered yeast populations. Proceedings of the National Academy of Sciences, 104(6):1877-1882, 2007.

R. D. Sloboda and L. Howard. Protein methylation in full length Chlamydomonas flagella. Cell motility and the cytoskeleton, 66(8):650-660, 2009.

K. L. Smith, B. H. Robison, J. J. Helly, R. S. Kaufmann, H. A. Ruhl, T. J. Shaw, B. S. Twining, and M. Vernet. Free-drifting icebergs: hot spots of chemical and biological enrichment in the Weddell Sea. science, 317(5837):478-482, 2007.

W. O. Smith, M. R. Dennett, S. Mathot, and D. A. Caron. The temporal dynamics of the flagellated and colonial stages of Phaeocystis antarctica in the Ross Sea. Deep-Sea Research Part II: Topical Studies in Oceanography, 50(3-4):605-617, 2003. ISSN 09670645. doi: 10.1016/S0967-0645(02)00586-6.

W. O. Smith Jr, R. F. Anderson, J. K. Moore, L. A. Codispoti, and J. M. Morrison. The US southern ocean joint global ocean flux study: an introduction to AESOPS. Deep Sea Research Part II: Topical Studies in Oceanography, 47(15-16):3073-3093, 2000a.

W. O. Smith Jr, J. Marra, M. R. Hiscock, and R. T. Barber. The seasonal cycle of phytoplankton biomass and primary productivity in the Ross Sea, Antarctica. Deep Sea Research Part II: Topical Studies in Oceanography, 47(15-16):3119-3140, 2000b.

W. O. Smith Jr, A. R. Shields, J. A. Peloquin, G. Catalano, S. Tozzi, M. S. Dinniman, and V. A. Asper. Interannual variations in nutrients, net community production, and biogeochemical cycles in the Ross Sea. Deep Sea Research Part II: Topical Studies in Oceanography, 53(8-10):815-833, 2006.

W. O. Smith Jr, P. N. Sedwick, K. R. Arrigo, D. G. Ainley, and A. H. Orsi. The ross sea in a sea of change. Oceanography, 25(3):90-103, 2012.

P. St-Laurent, P. Yager, R. Sherrell, S. Stammerjohn, and M. Dinniman. Pathways and supply of dissolved iron in the Amundsen Sea (Antarctica). Journal of Geophysical Research: Oceans, 122(9):7135-7162, 2017.

P. St-Laurent, P. Yager, R. Sherrell, H. Oliver, M. Dinniman, and S. Stammerjohn. Modeling the seasonal cycle of iron and carbon fluxes in the Amundsen Sea Polynya, Antarctica. Journal of Geophysical Research: Oceans, 124(3):1544-1565, 2019. 
M. Steinegger and J. Söding. Mmseqs2 enables sensitive protein sequence searching for the analysis of massive data sets. Nature biotechnology, 35(11):1026-1028, 2017.

R. W. Sterner. The role of grazers in phytoplankton succession. In Plankton ecology, pages 107-170. Springer, 1989.

C. Suffridge, L. Cutter, and S. A. Sañudo-Wilhelmy. A new analytical method for direct measurement of particulate and dissolved B-vitamins and their congeners in seawater. Frontiers in Marine Science, 4:11, 2017.

W. G. Sunda and S. A. Huntsman. Cobalt and zinc interreplacement in marine phytoplankton: Biological and geochemical implications. Limnology and Oceanography, 40(8):1404-1417, 1995.

B. Sundby, L. G. Anderson, P. O. Hall, Å. Iverfeldt, M. M. R. van der Loeff, and S. F. Westerlund. The effect of oxygen on release and uptake of cobalt, manganese, iron and phosphate at the sediment-water interface. Geochimica et Cosmochimica Acta, 50(6):1281-1288, 1986.

E. D. Swanner, N. J. Planavsky, S. V. Lalonde, L. J. Robbins, A. Bekker, O. J. Rouxel, M. A. Saito, A. Kappler, S. J. Mojzsis, and K. O. Konhauser. Cobalt and marine redox evolution. Earth and Planetary Science Letters, 390:253-263, 2014.

K. S. Swithers, A. K. Petrus, M. A. Secinaro, C. L. Nesbø, J. P. Gogarten, K. M. Noll, and N. C. Butzin. Vitamin $B_{12}$ synthesis and salvage pathways were acquired by horizontal gene transfer to the Thermotogales. Genome biology and evolution, 4(8): 842-851, 2012.

A. Tagliabue and K. R. Arrigo. Iron in the Ross Sea: 1. Impact on $\mathrm{CO}_{2}$ fluxes via variation in phytoplankton functional group and non-Redfield stoichiometry. Journal of Geophysical Research: Oceans, 110(C3), 2005.

A. Tagliabue, L. Bopp, and O. Aumont. Evaluating the importance of atmospheric and sedimentary iron sources to Southern Ocean biogeochemistry. Geophysical Research Letters, 36(13), 2009.

A. Tagliabue, T. Mtshali, O. Aumont, A. Bowie, M. Klunder, A. Roychoudhury, and S. Swart. A global compilation of dissolved iron measurements: focus on distributions and processes in the Southern Ocean. Biogeosciences, 9(6), 2012.

A. Tagliabue, O. Aumont, and L. Bopp. The impact of different external sources of iron on the global carbon cycle. Geophysical Research Letters, 41(3):920-926, 2014.

A. Tagliabue, O. Aumont, R. DeAth, J. P. Dunne, S. Dutkiewicz, E. Galbraith, K. Misumi, J. K. Moore, A. Ridgwell, E. Sherman, et al. How well do global ocean biogeochemistry models simulate dissolved iron distributions? Global Biogeochemical Cycles, 30(2):149-174, 2016. 
L. D. Talley. Descriptive Physical Oceanography: An Introduction. Academic press, 2011.

Y. Z. Tang, F. Koch, and C. J. Gobler. Most harmful algal bloom species are vitamin $\mathrm{B}_{1}$ and $\mathrm{B}_{12}$ auxotrophs. Proceedings of the national academy of sciences, 107(48): 20756-20761, 2010.

G. T. Taylor and C. W. Sullivan. Vitamin $B_{12}$ and cobalt cycling among diatoms and bacteria in antarctic sea ice microbial communities. Limnology and Oceanography, 53(5):1862-1877, 2008.

S. R. Taylor and S. M. McLennan. The continental crust: Its composition and evolution. Blackwell Scientific Publications, 11985.

B. M. Tebo, K. H. Nealson, S. Emerson, and L. Jacobs. Microbial mediation of Mn (ii) and $\mathrm{Co}$ (ii) precipitation at the $\mathrm{O}_{2} / \mathrm{H}_{2} \mathrm{~S}$ interfaces in two anoxic fjords. Limnology and Oceanography, 29(6):1247-1258, 1984.

T. F. Thingstad, L. Øvreås, J. K. Egge, T. Løvdal, and M. Heldal. Use of non-limiting substrates to increase size: a generic strategy to simultaneously optimize uptake and minimize predation in pelagic osmotrophs? Ecology Letters, 8(7):675-682, 2005.

C. Thuróczy, M. Boye, and R. Losno. Dissolution of cobalt and zinc from natural and anthropogenic dusts in seawater. Biogeosciences, 7:1927-1936, 2010.

K. M. Thyng, C. A. Greene, R. D. Hetland, H. M. Zimmerle, and S. F. DiMarco. True colors of oceanography: Guidelines for effective and accurate colormap selection. Oceanography, 29(3):9-13, 2016.

D. Tilman. Tests of resource competition theory using four species of Lake Michigan algae. Ecology, 62(3):802-815, 1981.

K. R. Timmermans, L. J. Gerringa, H. J. De Baar, B. Van Der Wagt, M. J. Veldhuis, J. T. De Jong, P. L. Croot, and M. Boye. Growth rates of large and small southern ocean diatoms in relation to availability of iron in natural seawater. Limnology and Oceanography, 46(2):260-266, 2001a.

K. R. Timmermans, J. Snoek, L. J. Gerringa, I. Zondervan, and H. J. de Baar. Not all eukaryotic algae can replace zinc with cobalt: Chaetoceros calcitrans (Bacillariophyceae) versus Emiliania huxleyi (Prymnesiophyceae). Limnology and Oceanography, 46(3):699-703, 2001b.

K. R. Timmermans, B. Van Der Wagt, and H. J. De Baar. Growth rates, halfsaturation constants, and silicate, nitrate, and phosphate depletion in relation to iron availability of four large, open-ocean diatoms from the Southern Ocean. Limnology and Oceanography, 49(6):2141-2151, 2004. 
P. D. Tortell, C. Payne, C. Gueguen, R. F. Strzepek, P. W. Boyd, and B. Rost. Inorganic carbon uptake by Southern Ocean phytoplankton. Limnology and Oceanography, 53 (4):1266-1278, 2008.

W. Van Boekel. Phaeocystis colony mucus components and the importance of calcium ions for colony stability. Marine Ecology-Progress Series, 87:301-301, 1992.

K. Van den Meersche, J. J. Middelburg, K. Soetaert, P. Van Rijswijk, H. T. Boschker, and C. H. Heip. Carbon-nitrogen coupling and algal-bacterial interactions during an experimental bloom: Modeling a ${ }^{13} \mathrm{C}$ tracer experiment. Limnology and Oceanography, 49(3):862-878, 2004.

C. M. van Hilst and W. O. Smith Jr. Photosynthesis/irradiance relationships in the Ross Sea, Antarctica, and their control by phytoplankton assemblage composition and environmental factors. Marine Ecology Progress Series, 226:1-12, 2002.

E. Vancaester, T. Depuydt, C. M. Osuna-Cruz, and K. Vandepoele. Systematic and functional analysis of horizontal gene transfer events in diatoms. bioRxiv, 2020.

M. J. Veldhuis, C. P. Brussaard, and A. A. Noordeloos. Living in a phaeocystis colony: a way to be a successful algal species. Harmful Algae, 4(5):841-858, 2005.

A. Verdy, M. Follows, and G. Flierl. Optimal phytoplankton cell size in an allometric model. Marine Ecology Progress Series, 379:1-12, 2009.

P. G. Verity, T. A. Villareal, and T. Smayda. Ecological investigations of blooms of colonial Phaeocystis pouchettii: Abundance, biochemical composition, and metabolic rates. Journal of Plankton Research, 10(2):219-248, 1988.

P. G. Verity, C. P. Brussaard, J. C. Nejstgaard, M. A. van Leeuwe, C. Lancelot, and L. K. Medlin. Current understanding of Phaeocystis ecology and biogeochemistry, and perspectives for future research. Biogeochemistry, 83(1-3):311-330, 2007.

A. G. Vitreschak, D. A. Rodionov, A. A. Mironov, and M. S. Gelfand. Regulation of the vitamin $\mathrm{B}_{12}$ metabolism and transport in bacteria by a conserved RNA structural element. RNA, 9(9):1084-1097, 2003.

C. Völker and D. A. Wolf-Gladrow. Physical limits on iron uptake mediated by siderophores or surface reductases. Marine Chemistry, 65(3-4):227-244, 1999.

M. R. Wadley, T. D. Jickells, and K. J. Heywood. The role of iron sources and transport for Southern Ocean productivity. Deep Sea Research Part I: Oceanographic Research Papers, 87:82-94, 2014.

T. Wagener, C. Guieu, R. Losno, S. Bonnet, and N. Mahowald. Revisiting atmospheric dust export to the Southern Hemisphere ocean: biogeochemical implications. Global Biogeochemical Cycles, 22(2):1-13, 2008. 
N. G. Walworth, F.-X. Fu, E. A. Webb, M. A. Saito, D. Moran, M. R. Mcllvin, M. D. Lee, and D. A. Hutchins. Mechanisms of increased Trichodesmium fitness under iron and phosphorus co-limitation in the present and future ocean. Nature communications, 7(1):1-11, 2016.

H.-C. Wang, B. Q. Minh, E. Susko, and A. J. Roger. Modeling site heterogeneity with posterior mean site frequency profiles accelerates accurate phylogenomic estimation. Systematic biology, 67(2):216-235, 2018.

S. Wang and J. K. Moore. Incorporating Phaeocystis into a Southern Ocean ecosystem model. Journal of Geophysical Research: Oceans, 116(C1), 2011.

S. Wang, D. Bailey, K. Lindsay, J. Moore, and M. Holland. Impact of sea ice on the marine iron cycle and phytoplankton productivity. Biogeosciences, 11(17):4713-4731, 2014.

B. A. Ward, S. Dutkiewicz, O. Jahn, and M. J. Follows. A size-structured food-web model for the global ocean. Limnology and Oceanography, 57(6):1877-1891, 2012.

F. Watanabe. Vitamin $\mathrm{B}_{12}$ sources and bioavailability. Experimental biology and medicine, 232(10):1266-1274, 2007.

A. Weber and M. Wettern. Some remarks on the usefulness of algal carotenoids as chemotaxonomic markers. Pigments in plants, pages 104-116, 1980.

C. Williams, A. Dupont, J. Loevenich, A. Post, J. Dinasquet, and P. Yager. Pelagic microbial heterotrophy in response to a highly productive bloom of Phaeocystis antarctica in the Amundsen Sea Polynya, Antarctica. Elem Sci Anth, 4, 2016.

E. H. Wintermute and P. A. Silver. Emergent cooperation in microbial metabolism. Molecular systems biology, 6(1), 2010.

A. Z. Worden, M. J. Follows, S. J. Giovannoni, S. Wilken, A. E. Zimmerman, and P. J. Keeling. Rethinking the marine carbon cycle: factoring in the multifarious lifestyles of microbes. Science, 347(6223):1257594, 2015.

M. Wu, J. S. P. McCain, E. Rowland, R. Middag, M. Sandgren, A. E. Allen, and E. M. Bertrand. Manganese and iron deficiency in Southern Ocean Phaeocystis antarctica populations revealed through taxon-specific protein indicators. Nature communications, 10(1):1-10, 2019.

L. L. Wurch, E. M. Bertrand, M. A. Saito, B. A. Van Mooy, and S. T. Dyhrman. Proteome changes driven by phosphorus deficiency and recovery in the brown tide-forming alga Aureococcus anophagefferens. PLoS One, 6(12), 2011.

P. L. Yager, R. Sherrell, S. Stammerjohn, H. Ducklow, O. Schofield, E. Ingall, S. Wilson, K. Lowry, C. Williams, L. Riemann, et al. A carbon budget for the Amundsen Sea Polynya, Antarctica: Estimating net community production and export in a highly productive polar ecosystem. Elementa: Science of the Anthropocene, 2016. 
L. Yuan-Hui and S. Gregory. Diffusion of ions in sea water and in deep-sea sediments. Geochimica et cosmochimica acta, 38(5):703-714, 1974.

K. Zengler and L. S. Zaramela. The social network of microorganisms: how auxotrophies shape complex communities. Nature Reviews Microbiology, 16(6): 383-390, 2018.

A. Zingone, G. Forlani, I. Percopo, and M. Montresor. Morphological characterization of Phaeocystis antarctica (Prymnesiophyceae). Phycologia, 50(6):650-660, 2011.

B. Zybailov, A. L. Mosley, M. E. Sardiu, M. K. Coleman, L. Florens, and M. P. Washburn. Statistical analysis of membrane proteome expression changes in Saccharomyces c erevisiae. Journal of proteome research, 5(9):2339-2347, 2006. 